\title{
Tidal Disruption of Stars by Supermassive Black Holes
}

\section{Citation}

Stone, Nicholas Chamberlain. 2013. Tidal Disruption of Stars by Supermassive Black Holes. Doctoral dissertation, Harvard University.

\section{Permanent link}

http://nrs.harvard.edu/urn-3:HUL.InstRepos:11041646

\section{Terms of Use}

This article was downloaded from Harvard University's DASH repository, and is made available under the terms and conditions applicable to Other Posted Material, as set forth at http:// nrs.harvard.edu/urn-3:HUL.InstRepos:dash.current.terms-of-use\#LAA

\section{Share Your Story}

The Harvard community has made this article openly available.

Please share how this access benefits you. Submit a story.

\section{Accessibility}


(c) 2013 - Nicholas Chamberlain Stone

All rights reserved. 


\title{
Tidal Disruption of Stars by Supermassive Black Holes
}

\begin{abstract}
This thesis presents theoretical results on the tidal disruption of stars by supermassive black holes (SMBHs). The multiwavelength flares produced by tidal disruption events (TDEs) have supernova-like luminosities, and associated relativistic jets can be visible to cosmological distances. TDEs probe the demography of quiescent SMBHs, and are natural laboratories for jet launching mechanisms and super-Eddington accretion.
\end{abstract}

The first chapter broadly surveys TDE physics. The second and third chapters estimate the TDE rate following gravitational wave (GW) recoil of a SMBH (after a SMBH binary merger). Immediately after GW recoil, the TDE rate increases, sometimes to $\sim 10^{-1}$ TDEs per year. This "burst" of TDE flares can provide an electromagnetic counterpart to low frequency GW signals, localizing sources and measuring cosmological parameters. Millions of years later, recoiled SMBHs wandering through their host galaxies will produce spatially offset TDEs at a rate which is likely detectable with the $L S S T$.

In the fourth chapter, we show that standard estimates for $\Delta \epsilon$, the energy spread of TDE debris, are wrong, sometimes by orders of magnitude. Correcting this error reduces the observability of many TDEs. We introduce a new analytic model for tidal disruption, calculate $\Delta \epsilon$ 's dependence on stellar spin, estimate general relativistic corrections to $\Delta \epsilon$, and quantify the GW signal generated from tidal compression. The fifth chapter presents 
hydrodynamical simulations of TDE debris circularization, focusing on eccentric, rather than parabolic, orbits. General relativistic precession drives debris circularization, in contrast to past simulations using smaller black holes.

In the sixth chapter, we show that TDE light curves can constrain or measure SMBH spins, as Lense-Thirring torques produce quasiperiodic variability in disk emission. Precession of a relativistic jet could also measure SMBH spin, and we apply our model to the relativistic Swift 1644+57 TDE. The seventh chapter considers the disruption of neutron stars (NSs) by stellar mass black holes (BHs) or other NSs. Jet precession in associated short-hard gamma ray bursts is uniquely possible for NS-BH (not NS-NS) mergers. We quantify typical precession amplitudes and periods, and calculate their time evolution. If disk viscosities are relatively low, electromagnetic observations alone could distinguish NS-BH from NS-NS mergers. 


\section{Contents}

$\begin{array}{ll}\text { Abstract } & \text { iii }\end{array}$

$\begin{array}{ll}\text { Acknowledgments } & \text { ix }\end{array}$

Dedication $\quad$ xii

1 Introduction $\quad 1$

1.1 SMBHs in our Universe . . . . . . . . . . . . . . . . . . . 1

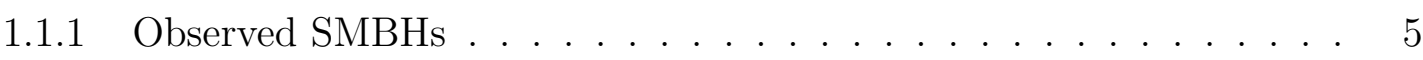

1.1.2 SMBH Formation and Growth . . . . . . . . . . . . . 7

1.1.3 Stellar Tidal Disruption _. . . . . . . . . . . . . . . . 10

1.2 Tidal Disruption Basics . . . . . . . . . . . . . . . . . . . . . . 11

1.2.1 The Newtonian Picture . . . . . . . . . . . . . . . . 11

1.2.2 The Role of General Relativity . . . . . . . . . . . . . . . . 16

1.3 Tidal Disruption Event Rates . . . . . . . . . . . . . . . . . 19

1.3.1 Two Body Relaxation . . . . . . . . . . . . . . . . . 19

1.3.2 Resonant Relaxation . . . . . . . . . . . . . . . . . . . . . 22

1.3.3 Alternative Relaxational Mechanisms . . . . . . . . . . . . . . . 25

1.4 Dynamics of Disruption . . . . . . . . . . . . . . . . . 27

1.4.1 Vertical Collapse and Crunch: (Semi-)Analytic . . . . . . . . . . . 28

1.4.2 Vertical Collapse and Crunch: Hydrodynamic Simulations . . . . . 33 
CONTENTS

1.4.3 Debris Expansion and Circularization . . . . . . . . . . . . 35

1.5 Accretion of Circularized Debris . . . . . . . . . . . . . . . 37

1.6 Observed Flares . . . . . . . . . . . . . . . . . . . . . . 41

1.6.1 X-ray and UV Observations . . . . . . . . . . . . . . . . . 41

1.6.2 Optical Detection of TDEs . . . . . . . . . . . . . . 43

1.6.3 Relativistic TDEs . . . . . . . . . . . . . . . . . . 44

2 Prompt Tidal Disruption of Stars as an Electromagnetic Signature of $\begin{array}{ll}\text { Supermassive Black Hole Coalescence } & 47\end{array}$

2.1 Introduction . . . . . . . . . . . . . . . . . . . . 48

2.2 Physics of the Loss Cone . . . . . . . . . . . . . . . . . . . . 51

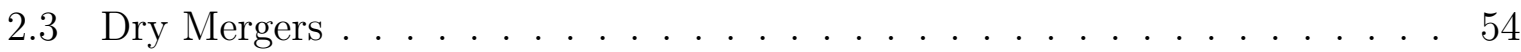

2.4 Wet Mergers . . . . . . . . . . . . . . . . . . 57

2.5 Other Considerations . . . . . . . . . . . . . . . . . . . . 60

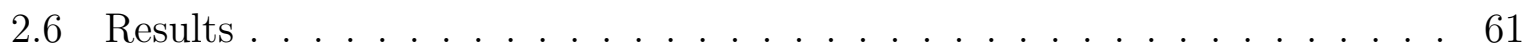

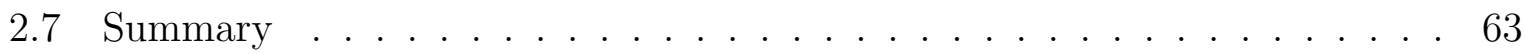

3 Tidal Disruption Flares of Stars From Moderately Recoiled Black Holes 65

3.1 Introduction . . . . . . . . . . . . . . . . . 66

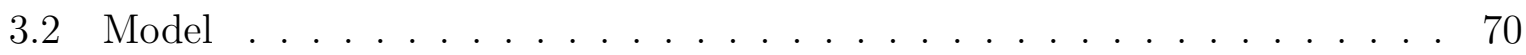

3.2.1 Black Hole Kicks . . . . . . . . . . . . . . . . . . . . 70

3.2.2 Tidal Disruption Physics . . . . . . . . . . . . . . . . 73

3.2 .3 Host Galaxy Structure . . . . . . . . . . . . . . . . . . 77

3.2.4 Interactions with the Galaxy . . . . . . . . . . . . . 85

3.2.5 Interactions with the Bound Cloud . . . . . . . . . . . . . 89

3.2.6 Observability of Recoil-Induced TDEs . . . . . . . . . . . . . . 93

3.3 TDE Rate . . . . . . . . . . . . . . . . . . . . . . . . 94

3.4 Results and Discussion . . . . . . . . . . . . . . . . 98 
CONTENTS

3.5 Conclusions . . . . . . . . . . . . . . . . . . . . 112

4 Consequences of Strong Compression in Tidal Disruption Events 114

4.1 Introduction . . . . . . . . . . . . . . . . . . . 115

4.2 Dynamical Energy Spread . . . . . . . . . . . . . . . . . . 118

4.3 Free Solutions, and Free Collapse . . . . . . . . . . . . . . . . . 121

4.4 Total Vertical Collapse and Bounce . . . . . . . . . . . . . . 136

4.5 Desynchronization . . . . . . . . . . . . . . . . . 141

4.5.1 Desynchronized Free Solutions . . . . . . . . . . . . . . . . 142

4.5.2 Validity of Free Solutions in One Dimension . . . . . . . . . . . . 145

4.5.3 Validity of Free Solutions in Three Dimensions . . . . . . . . . . . . 147

4.6 General Relativistic Corrections . . . . . . . . . . . . . . . . 153

4.7 Gravitational Waves . . . . . . . . . . . . . . . . . . 157

4.8 Observational Implications . . . . . . . . . . . . . . . . . . . . . 162

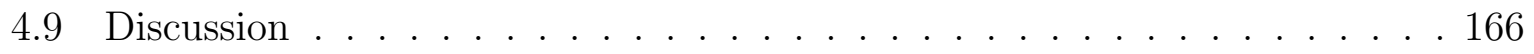

5 Finite, Intense Accretion Bursts from Tidal Disruption of Stars on $\begin{array}{lr}\text { Bound Orbits } & 172\end{array}$

5.1 Introduction . . . . . . . . . . . . . . . . . . . 173

5.2 Numerical method ....................... 176

5.2.1 Formation of polytropes . . . . . . . . . . . . . 177

5.2 .2 Initial configuration . . . . . . . . . . . . . . . . . . . 178

5.2.3 Treatment of relativistic precession . . . . . . . . . . . 180

5.2 .4 Numerical models . . . . . . . . . . . . . . . . . . 183

5.3 Tidal disruption of stars on bound orbits . . . . . . . . . . . . 187

5.3.1 Eccentric tidal disruption . . . . . . . . . . . . . . . 192

5.3.2 Accretion disk formation . . . . . . . . . . . . . . . . . 198

5.4 Sources of Eccentric TDEs . . . . . . . . . . . . . . . . . . . 207 
CONTENTS

5.5 Summary \& Discussion . . . . . . . . . . . . . . . . . . . . . . . 212

6 Observing Lense-Thirring Precession in Tidal Disruption Flares 220

6.1 Introduction . . . . . . . . . . . . . . . . . . . . . . . . . . . . 221

6.2 Spin Evolution of a Tilted Disk . . . . . . . . . . . . . . . . . 222

6.3 Angular Momenta of Returning Debris Streams. . . . . . . . . . . . . 226

6.4 Observational Implications. . . . . . . . . . . . . . . . . . 230

7 Pulsations in Short GRBs from Black Hole-Neutron Star Mergers 233

7.1 Introduction . . . . . . . . . . . . . . . . . . . . . . . 234

7.2 Disk Precession . . . . . . . . . . . . . . . . . . . 236

7.3 Progenitor Binaries . . . . . . . . . . . . . . . . . . . . . . . 244

7.4 Distributions . . . . . . . . . . . . . . . . . . . . 247

7.5 Discussion . . . . . . . . . . . . . . . . . . 257

8 Conclusions and Future Directions 261

$\begin{array}{ll}\text { References } & 265\end{array}$

A The Parabolic Hill Equations 283

B Principal Axes of Free Solutions 285

$\begin{array}{ll}\text { C Innermost Stable Spherical Orbits } & 287\end{array}$

D Post-Newtonian Merger Treatment 289 


\section{Acknowledgments}

This thesis would not have been possible without help from the many fantastic people who gave me the academic, technical, and personal support I needed during the last five years. I will do my best to offer some much deserved thanks in this space.

I owe an immense debt to my family; to my parents most of all. From as early as I can remember, my parents kept me supplied with a continuous stream of science (and, perhaps more importantly, science fiction ${ }^{1}$ ) books. Later in life, they helped and encouraged me to succeed academically, but with a light enough touch that I always felt supported, not pressured. My sisters, too, have always been an important part of my life, and as an older brother it's been a pleasure to watch their successes - Madeleine, with her research, and Jasmine, with her languages - during my intermittent struggles in graduate school. I am sure there will be many more of these in the future. I also must thank my wonderful girlfriend, Alicia Breakey, for putting up with the many unique challenges of dating an astrophysicist.

Here at Harvard, my advisor Avi Loeb has played the greatest role in my development as a scientist. I owe Avi a huge debt of gratitude for his mentorship and collaboration over the last five years. One great thing about working with Avi is his openness to new ideas - our first project together was based on a question I asked at his group meeting during my first semester at Harvard. Whenever I have gotten stuck on a research project, Avi has always made the time to help out. Since he always has a fresh

\footnotetext{
${ }^{1}$ Although the tidal disruption of stars by black holes - the subject of this thesis - may seem like an esoteric topic, it's actually one I first read about at age 13 in a science fiction book my mother checked out of the library.
} 


\section{CHAPTER 0. ACKNOWLEDGMENTS}

way of looking at a problem (or connecting it to another topic I wasn't previously aware of), these meetings usually cleared up my problems quickly. Most of what I know about being an actual, practicing scientist I have learned from Avi.

My other collaborators in research have also been great mentors and sources of inspiration. I wish to thank Re'em Sari for suggesting new ways to look at subjects I had thought I was already familiar with. Re'em's physical insights resolved many questions I couldn't find other ways to answer. Edo Berger has been a great collaborator, and also a fantastic connection to the world of observational astronomy. Theorists sometimes can wander off into corners or otherwise get disconnected from the real world, so I know I have benefitted greatly from talking to Edo about the latest discoveries in time domain or high energy astronomy. My most recent collaborator, Kimi Hayasaki, has brought reliable numerical expertise to our work, which is a great complement to my typically more analytical research.

I would also like to thank my thesis committee, which is chaired by Alicia Soderberg and has comprised Josh Grindlay, Ramesh Narayan, Avi Loeb, and Enrico Ramirez-Ruiz. Reading a thesis is a lot to ask of someone, so I'm very grateful to have received advice from these experts in high-energy and accretion astrophysics.

Last but not least, I owe many thanks to the other Harvard astronomy graduate students, who through conversation, hikes, and bad movies have made my time here great fun. Robert Harris deserves special commendation for five years of sharing an office with me. I would also like to thank Ryan O'Leary, Diego Munoz, Laura Blecha, Sarah Ballard, Paul Torrey, Zach Berta, Wen-fai Fong, Greg Snyder, Bob Penna, Jon Bittner, Gongjie Li, and many others. Many friendships I have made here have followed me 


\section{CHAPTER 0. ACKNOWLEDGMENTS}

outside of Cambridge. Two adventures I will never forget were with great friends who happened to be fellow graduate students: Sarah Rugheimer and Maggie McLean, with whom I shared a memorable winter solstice tour of Iceland, and Tony Pan, who may never forgive my driving during an eventful journey around Israel, Jordan and Egypt. 
To my parents, Eve and Peter 


\section{Chapter 1}

\section{Introduction}

\subsection{SMBHs in our Universe}

From an observational perspective, supermassive black holes (SMBHs) are nearly ubiquitous in nearby galactic nuclei. These massive objects $\left(\sim 10^{5-10} M_{\odot}\right)$ generally reside at the center of their host galaxy's stellar bulge, and can be observed either through the luminous accretion of gas, or via gravitational interactions with surrounding stellar populations. In this thesis, we focus on strong, disruptive tidal encounters between SMBHs and nearby stars, but SMBHs themselves are objects of much intrinsic interest, as we outline in this introductory subsection.

Mathematically, black holes are very simple objects, characterized by a mere three numbers (Wald 1984, chapter 12.3): mass, spin, and charge ${ }^{1}$. For this reason, measuring the demographics of black holes is appealingly straightforward at a conceptual level -

\footnotetext{
${ }^{1}$ However, electric charge is expected to be negligibly small for any astrophysical black hole.
} 


\section{CHAPTER 1. INTRODUCTION}

but the devil lies in the details. Although a variety of techniques can be used to measure the masses of SMBHs, the only effective approaches at large distances involve the luminous accretion flows present around $\sim 1 \%$ of SMBHs. This introduces a potentially serious bias into SMBH mass measurements. Such bias is more pronounced for spin measurements, which for even the nearest SMBHs can only be performed by taking advantage of surrounding accretion flows. Probing the universe's "silent majority" of quiescent SMBHs is an inherently difficult task, but the many scientific motivations for studying SMBHs should encourage us to find ways to do this.

The most fundamental motivation for studying SMBHs is that as strongly relativistic objects, they offer a laboratory to probe and test general relativity (GR). Observations of Sgr A*, the SMBH in the center of the Milky Way, have provided some of the strongest evidence to date for the existence of event horizons (Broderick et al. 2009). Future observations of SgrA* (and M87's SMBH) with Very Long Baseline Interferometry will likely provide direct imaging of event horizons and the gas flows around them (Broderick \& Loeb 2005; Doeleman et al. 2009; Broderick \& Loeb 2009). Measurements of SMBH spin in distant galaxies using relativistically broadened iron lines (Reynolds et al. 1999; Reynolds 2013) provide a zeroth-order check on the cosmic censorship hypothesis ${ }^{2}$, and perhaps a more detailed test of the no-hair theorem as well (Johannsen \& Psaltis 2012). Future detections of stellar motion in our own Galactic Center may enable a direct test of GR by measuring the mass, spin, and quadrupole moment ${ }^{3}$ of Sgr A* (Merritt et al. 2010). Such a test could be facilitated by the discovery of a pulsar in the Galactic Center

\footnotetext{
${ }^{2}$ Which would be falsified by the discovery of a single SMBH with super-extremal spin.

${ }^{3}$ The black hole quadrupole moment is uniquely determined by mass and spin in GR, but not in many alternative theories of gravity (Johannsen \& Psaltis 2010).
} 


\section{CHAPTER 1. INTRODUCTION}

(Pfahl \& Loeb 2004). SMBHs can also be used to probe other questions in fundamental physics; for example, general relativistic Kerr black holes are believed to possess a semiclassical instability to scalar fields in specific (low) energy ranges. The existence of spinning SMBHs in the universe places valuable constraints on massive photons (Pani et al. 2012) and other exotic light particles.

SMBHs also seem to play an integral role in the growth and evolution of galaxies. Specifically, the coevolution of galaxies and their central SMBHs has been observationally established by scaling relations linking the SMBH mass to a variety of galaxy parameters (Kormendy \& Richstone 1995; Ferrarese \& Merritt 2000; Ferrarese 2002; Tremaine et al. 2002; Bandara et al. 2009; Burkert \& Tremaine 2010). These relationships are sometimes disputed (Kormendy \& Bender 2011, for example) and are somewhat surprising, given that the SMBH mass is typically $\sim 1 / 500$ the total stellar mass of the bulge it resides in (Marconi \& Hunt 2003). The standard explanation, at least for the well-known $M_{\mathrm{BH}}-\sigma$ relation, is that the gas accretion processes which grow a SMBH drive strong outflows that can self-regulate star formation through either energy or momentum deposition (Silk \& Rees 1998; Wyithe \& Loeb 2003; Di Matteo et al. 2005; Hopkins et al. 2006). Alternative explanations, such as the central limit theorem (Peng 2007; Jahnke \& Macciò 2011), exist as well, however. If there is a causal link between SMBH accretion and star formation, understanding the demographics and growth history of SMBHs becomes a crucially important step in understanding many other aspects of astrophysics.

The extreme accretion environments surrounding a minority of SMBHs are also sites of intrinsic astrophysical interest. These active galactic nuclei (AGN) are important targets for observations at all electromagnetic wavelengths. Although a full review of AGN phenomenology and accretion physics is well beyond the scope of this paper - 


\section{CHAPTER 1. INTRODUCTION}

indeed, it is the subject of many books, such as Krolik (1999) and Beckmann \& Shrader (2012) - we do wish to mention a few points of interest. Because of their extreme luminosities, AGN are some of the most distant objects observed in the universe (Fan et al. 2003; Mortlock et al. 2011). Relativistic jets launched by AGN are also a possible source for observed ultra high energy cosmic rays (Biermann \& Strittmatter 1987; Pierre Auger Collaboration et al. 2008; Farrar \& Gruzinov 2009), whose origin is an important open question in particle astrophysics.

As a result of their compactness, large masses, and abundance (Volonteri et al. 2003), SMBH binaries (SMBHBs) are key sources for gravitational wave (GW) astronomy, detectable both by space-based, low frequency GW interferometers (Jennrich 2009; Amaro-Seoane et al. 2012b), and by pulsar timing arrays (Sazhin 1978; Jenet et al. 2004; Sesana et al. 2009). The coalescence of a SMBHB is an extremely energetic event, as numerical relativity simulations indicate that up to $\sim 10 \%$ of the binary rest mass energy can be radiated in GWs during the final orbits and plunge (Pretorius 2005). Small anisotropies in this final burst of gravitational radiation give a recoil kick to the merged SMBH, typically $\sim 100 \mathrm{~km} \mathrm{~s}^{-1}$ (Lousto et al. 2010a), but up to $\approx 5000 \mathrm{~km} \mathrm{~s}^{-1}$ (Campanelli et al. 2007; Lousto \& Zlochower 2011, 2012). The instantaneous GW luminosity of such a merger can exceed the electromagnetic luminosity of the entire observable universe (Schnittman 2011), and in the right mass range $\left(\sim 10^{5-6} M_{\odot}\right)$ would be detectable by a $L I S A$-like instrument to any realistic redshift (Amaro-Seoane et al. 2012b, Fig. 3).

The detection of LISA-band GWs would lead to many scientific opportunities. By observing mergers of SMBHBs across cosmic time, SMBH demography could be studied in ways that lack the biases of AGN observations. The spins and redshifted masses of 


\section{CHAPTER 1. INTRODUCTION}

the merging SMBHs could be measured to high precision, with errors $\lesssim 1 \%$ (Klein et al. 2009; Amaro-Seoane et al. 2012b); if the merger is accompanied by an electromagnetic counterpart (to break the GW degeneracy between redshift and luminosity distance), these "standard sirens" could measure cosmological parameters independent of the standard cosmic distance ladder (Schutz 1986; Holz \& Hughes 2005). Alternatively, if a low frequency GW observatories measures the GW-driven inspiral of a stellar mass compact object into a SMBH, the SMBH's Kerr spacetime could be mapped out in high precision (Ryan 1995). This would both measure the SMBH mass and spin, and test extensions of GR (Amaro-Seoane et al. 2007; Sopuerta 2010).

To summarize, both electromagnetic and GW observations of SMBHs offer immense scientific value. SMBHs are probes of strong-field GR, and also objects that play an important role in many different areas of astrophysics. In the next subsection, we briefly outline the most prominent of the many techniques used today to directly identify and measure SMBHs in our universe.

\subsubsection{Observed SMBHs}

The nearest SMBH, Sgr A*, resides in the center of the Milky Way. The advent of adaptive optics technology has allowed all six orbital elements to be measured for an entire population of stars, the S stars, on tight orbits around Sgr A* (Ghez et al. 2005; Gillessen et al. 2009). Until recently, the most tightly bound S-star known was S0-2 (Schödel et al. 2002), which orbited with a period of 16 years. Very recently, the much dimmer star S0-102 has been discovered to lie on an 11.5 year orbit (Meyer et al. 2012). The measurement of S star orbital properties has allowed the mass and distance of Sgr 
A* to be measured with high precision (Ghez et al. 2008) - although much closer S stars will need to be found in order to observe relativistic precession effects, let alone test GR (Merritt et al. 2010).

Although Sgr A* is the nearest SMBH, its extremely underluminous nature (Baganoff et al. 2003) prevented it from being the first detected by astronomers. The first strong evidence for astrophysical black holes was inferred by Lynden-Bell (1969), following earlier work by Hoyle \& Fowler (1963) and Salpeter (1964). These theoretical papers were written in response to the first discoveries of quasars (Hazard et al. 1963; Schmidt 1963), which are found in much larger numbers today. AGN observations can provide SMBH mass estimates through photoionization modeling (Wandel et al. 1999) or reverberation mapping (Kaspi et al. 2000) of broad emission line regions, among other methods. As mentioned previously, observations of AGN can also measure SMBH spin through relativistically broadened iron lines (Reynolds 2013), whose profiles depend sensitively on the location of the innermost stable circular orbit (ISCO).

In the nearest galaxies, SMBH masses can be measured dynamically (albeit not as directly as for Sgr $A^{*}$ ). This is generally accomplished using integrated stellar kinematic data (Gebhardt et al. 2000, for example) and comparing to three-integral dynamical models (Schwarzschild 1979) with ranges of SMBH masses. A different dynamical technique uses stimulated emission from water masers in SMBH accretion disks, which can provide both distance (Herrnstein et al. 1999) and mass (Greenhill et al. 1997) estimates for extragalactic SMBHs; other gas dynamical mass estimates can be used as well.

Taken together, the above techniques have taught us that SMBHs are nearly 
CHAPTER 1. INTRODUCTION

ubiquitous in nearby galactic nuclei (Kormendy \& Richstone 1995; Ferrarese \& Ford 2005). The SMBH mass function has been characterized using combinations of these methods, as well as host galaxy scaling relations (Franceschini et al. 1998; Shankar et al. 2004; Hopkins et al. 2007; Greene \& Ho 2007). Its $z=0$ peak is near $M_{\mathrm{BH}} \sim 10^{6}-10^{7} M_{\odot}$, with a gradual decline at higher and perhaps lower masses (the low mass end is limited by small sample sizes and observational selection effects). The SMBH spin function seems to indicate a bias towards rapidly spinning SMBHs, but is limited by small number

statistics (with roughly 20 measurements so far), stronger selection effects, and a lack of independent checks on relativistic line broadening, the primary method of measurement (Reynolds 2013).

\subsubsection{SMBH Formation and Growth}

Despite the wealth of observational data on nearby SMBHs, and distant AGN, the origin of massive black holes in the universe is far from clear. Studying their growth and accretion history offers a few basic constraints. Soltan's argument (Soltan 1982; Yu \& Tremaine 2002) indicates that typical SMBHs grow primarily through radiatively efficient accretion of gaseous matter (rather than through mergers with other SMBHs, or through the capture of stellar mass compact objects). However, the discovery of extreme quasars at high redshift (Mortlock et al. 2011, for example) suggests that at least some SMBHs were capable of reaching enormous sizes $\left(\gtrsim 10^{9} M_{\odot}\right)$ in the first Gyr of the universe. If their growth occurred due to Eddington-limited gas accretion capable of spinning up the SMBHs, the seed black hole mass they grew from must have been quite large, in the range of $\gtrsim 10^{4} M_{\odot}$ (Volonteri \& Rees 2006). Ejection of SMBHs via GW 


\section{CHAPTER 1. INTRODUCTION}

recoil makes the observed high- $z$ sample even more challenging to explain, although the problem can be ameliorated by invoking super-Eddington accretion, "chaotic accretion" of randomly aligned gas, or radiatively inefficient accretion flows (Volonteri \& Rees 2006). Many different mechanisms have been proposed for the formation of SMBH seeds (Sesana 2012), of which three stand out as particularly promising:

- Population III stellar remnants: the first generation of stars have traditionally been estimated to reach sizable masses, hundreds of times as large as the Sun. The lack of metal line opacity in their atmospheres would limit mass loss in line driven winds, allowing for extreme supernovae that can produce black holes with $M_{\mathrm{BH}} \sim 10^{2} M_{\odot}$ (Madau \& Rees 2001; Volonteri et al. 2003). Although the production of these SMBH seeds is likely more reliable than the other mechanisms listed here, the black holes produced from Population III stars are relatively small, and might have problems growing into observed high- $z$ SMBHs assuming Eddington-limited accretion. Recent theoretical work indicating that Population III stars can fragment during formation into lower mass objects may worsen this problem (Stacy et al. 2010).

- Relativistic star cluster instability: at extreme densities, a population of stars or compact remnants can begin undergoing runaway collisions, culminating in the direct production of an intermediate mass black hole (IMBH), the ejection of all massive compact remnants, or the formation of a supermassive star. If the latter object forms, its lifetime is limited by both its available fuel and general relativistic instability, leading to slightly delayed IMBH formation. The densities required to begin this collisional runaway arise from the Spitzer instability (Spitzer 1987): 


\section{CHAPTER 1. INTRODUCTION}

the negative heat capacity of stellar systems means that the heaviest stars will sink to the center and become more tightly bound in a "core collapse" process. If the core collapse timescale is less than the lifetime of the most massive stars, the collisional runaway will begin and form a supermassive star (Devecchi \& Volonteri 2009; Devecchi et al. 2010, 2012). IMBHs formed in this way will have masses $M_{\mathrm{BH}} \sim 10^{3} M_{\odot}$, somewhat larger than in the prior scenario. Alternatively, if the core collapse timescale is longer than the relevant stellar lifetimes, then the cluster's densest central regions will be composed of stellar mass black holes. These black holes will typically eject themselves from the cluster (through strong scatterings and post-merger GW recoil), preventing IMBH formation. However, collisions in a cluster of stellar mass black holes can still produce an IMBH if their core collapse is mediated by a large-scale gas inflow that deepens the cluster potential (Davies et al. 2011). An IMBH produced in this way could possess an initial mass $M_{\mathrm{BH}} \sim 10^{5} M_{\odot}$.

- Direct collapse: at high redshifts, a large mass of primordial gas can collapse into a large SMBH seed, either directly (Loeb \& Rasio 1994; Bromm \& Loeb 2003; Mayer et al. 2010) or through a short-lived, intermediate "quasistar" phase (Begelman et al. 2006, 2008). This mechanism produces the largest SMBH seeds, with $M_{\mathrm{BH}} \gtrsim 10^{5} M_{\odot}$ - but see Begelman et al. (2008) for a scenario in which $M_{\mathrm{BH}}$ is limited to $\sim 10^{3}-10^{4} M_{\odot}$. However, direct collapse requires somewhat delicate conditions to operate: if the dense gas is able to cool through molecular $\left(\mathrm{H}_{2}\right)$ or metal line emission, it will fragment into stars instead. Even slight metal enrichment from Population III stars could suffice to deactivate this channel of black hole formation (Volonteri et al. 2008). 


\section{CHAPTER 1. INTRODUCTION}

Although demographics seem like the best tool to distinguish between these hypotheses, current observations of SMBHs do not yet strongly distinguish between SMBH formation mechanisms or patterns of growth. As our sample of SMBH spin measurements expands, we will increasingly be able to distinguish between growth mechanisms for SMBHs (Volonteri et al. 2005; Berti \& Volonteri 2008). Spin measurements can also strengthen or relax the constraints that the present day SMBH mass function places on black hole seed masses. However, the limitations of current observational techniques will contribute significant uncertainty to any inferences drawn from SMBH demographic data. In particular, our measurements of SMBH masses and spins are biased towards the largest AGN; most SMBHs in the universe are smaller, and undergoing much weaker accretion.

More exotic future efforts could improve our understanding of SMBH demographics. In particular, low frequency GW astronomy will let us measure masses and spins of quiescent SMBHs out to large redshifts. Unfortunately, the funding situation for LISA-like instruments is quite uncertain, and pulsar timing arrays are unlikely to resolve any but the most massive SMBH binaries.

\subsubsection{Stellar Tidal Disruption}

There is one technique accessible to today's observers which enables the demographics of quiescent SMBHs to be measured. Over long timescales, stellar dynamics in galactic nuclei are collisional: the orbital parameters of individual stars change due to perturbations from other stars, compact stellar remnants, or more massive objects. Infrequently, a star can be perturbed onto an almost radial trajectory, and pass so close 
to the central SMBH that it is shredded by tidal forces (Hills 1975; Lidskii \& Ozernoi 1979; Rees 1988). The fallback of stellar debris onto the SMBH will create a transient accretion disk, which in turn powers a luminous high-energy flare.

Roughly twenty of these tidal disruption events (TDEs) have been seen over the last two decades, and our theoretical picture of them is steadily improving. This thesis describes our work to better understand TDEs: their rates (Stone \& Loeb 2011, 2012b), their dynamics (Hayasaki et al. 2012; Stone et al. 2012b), and their radiative emission (Stone \& Loeb 2012a). We also have examined an analogous scenario, in which a neutron star is tidally disrupted by a stellar mass black hole, producing a short gamma ray burst (Stone et al. 2012a). The remainder of this introduction will outline the current understanding of stellar tidal disruption, from both theoretical and observational perspectives. The study of TDEs is rapidly accelerating due to the wealth

of new and unexpected observations over the last few years. However, if the potential of TDEs to trace SMBH demography in a less (or differently) biased way than AGN-based techniques is to be realized, much theoretical progress remains to be made. In the following introductory sections, we place special emphasis on the many important open questions which await solution.

\subsection{Tidal Disruption Basics}

\subsubsection{The Newtonian Picture}

Unlucky stars in galactic nuclei are occasionally perturbed onto nearly radial orbits. Although these trajectories are doomed by their interaction with the (strongly relativistic) 


\section{CHAPTER 1. INTRODUCTION}

central SMBH, much of the relevant physics can be derived in a Newtonian framework, which we employ here. If a star of mass $M_{*}$ and radius $R_{*}$ orbits a black hole of mass $M_{\mathrm{BH}}$ with orbital pericenter, $R_{\mathrm{p}}$, less than the tidal radius

$$
R_{\mathrm{t}}=R_{*}\left(M_{\mathrm{BH}} / M_{*}\right)^{1 / 3}
$$

the star will be tidally disrupted (Hills 1975). To within a factor of order unity, this is the same as the Roche radius or Hill radius seen in other areas of astrophysics. The strength of the disruption event can be measured by a dimensionless inverse impact parameter,

$$
\beta=R_{\mathrm{t}} / R_{\mathrm{p}}
$$

which measures the strength of the tidal encounter (alternatively, some papers in the literature parametrize the strength of tidal encounters with $\eta_{\text {tidal }}=\beta^{-3 / 2}$ ). Because the horizon of a non-spinning SMBH grows linearly with $M_{\mathrm{BH}}$,

$$
R_{\mathrm{s}}=\frac{2 G M_{\mathrm{BH}}}{c^{2}}
$$

while the tidal radius only grows as $M_{\mathrm{BH}}^{1 / 3}$, above a critical mass (sometimes known as the Hills mass)

$$
M_{\mathrm{Hills}}=1.1 \times 10^{8} M_{\odot} r_{*}^{3 / 2} m_{*}^{-1 / 2}
$$

stars will be swallowed by the horizon prior to tidal disruption, making such events uninteresting from an electromagnetic point of view - although gravitational wave (GW) signals can still be emitted. In this and subsequent formulae, $r_{*}=R_{*} / R_{\odot}$ and $m_{*}=M_{*} / M_{\odot}$. We illustrate the region of parameter space accessible to TDEs in Fig. 1.1.

When a star is tidally disrupted, its constituent gas begins moving on roughly 


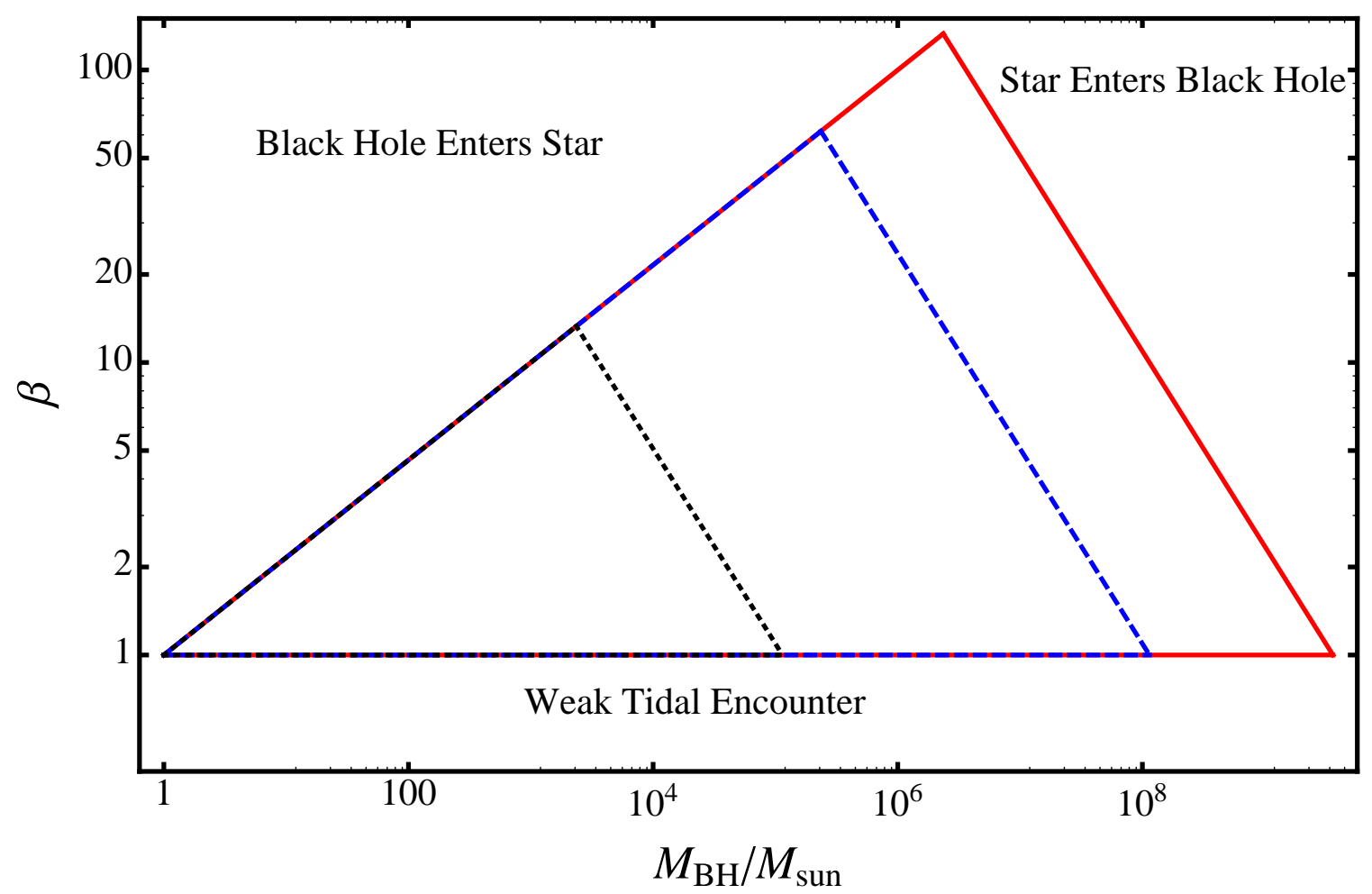

Figure 1.1.- : The parameter space of tidal disruption: only a star within its respective triangle can be tidally disrupted by a black hole (Luminet \& Pichon 1989). The three triangles are calculated for solar-type stars (blue, dashed), red giants with $M_{*}=M_{\odot}$, $R_{*}=10 R_{\odot}\left(\right.$ red, solid), and white dwarfs with $M_{*}=M_{\odot}, R_{*}=10^{-2} R_{\odot}$ (black, dotted). Below the triangles, $\beta<1$ and tidal encounters are not fully disruptive. In the upper left of the diagram, $R_{*}>R_{\mathrm{p}}$ and deeply plunging orbits around small black holes lead to engulfment of the black holes by the stars. In the upper right of the diagram, the stars encounter a black hole past their Hills mass limit and are swallowed whole. Using these approximate formulae, white dwarfs, solar type stars and red giants can reach maximum $\beta$ values of 13,62 , and 133 , respectively. 


\section{CHAPTER 1. INTRODUCTION}

ballistic trajectories, with a spread of specific orbital energies that (approximately) "freezes in" at the moment of disruption, i.e. when the orbital radius $R=R_{\mathrm{t}}$. This spread in orbital energies arises because at the moment of disruption, leading portions of the star sit deeper in the SMBH potential well than the trailing portions, which are in shallower regions of the SMBH potential. Taking the Taylor expansion of the SMBH's potential at the star's position gives the approximate spread in debris specific energy (Rees 1988),

$$
\Delta \epsilon=\frac{G M_{\mathrm{BH}} R_{*}}{R_{\mathrm{t}}^{2}}
$$

In much past literature this was incorrectly written as $G M_{\mathrm{BH}} R_{*} / R_{\mathrm{p}}^{2}$; Chapter 4 goes into significantly more detail on this disagreement. Tidal compression of the star orthogonal to the orbital plane (Carter \& Luminet 1982, 1983) redistributes energies of individual fluid elements, but likely does not change the overall spread (Stone et al. 2012b; Guillochon \& Ramirez-Ruiz 2013).

Most stars tidally disrupted in realistic galactic nuclei approach the SMBH on nearly parabolic, zero energy orbits whose apocenters lie at parsec scales (Magorrian \& Tremaine 1999; Wang \& Merritt 2004); we can therefore write a hierarchy of relevant specific energies, with the center of mass orbital specific energy $\epsilon_{\text {orb }} \ll \epsilon_{*} \ll \Delta \epsilon$. Here $\epsilon_{*}=G M_{*} / R_{*}$ is the approximate specific binding energy of the star prior to disruption. Since $\epsilon_{\text {orb }} \ll \Delta \epsilon$, the value of $\Delta \epsilon$ sets the fallback timescale for the most tightly bound debris,

$$
t_{\text {fall }}=3.5 \times 10^{6} \mathrm{~s}_{6}^{1 / 2} m_{*}^{-1} r_{*}^{3 / 2} .
$$

Here $M_{6}=M_{\mathrm{BH}} /\left(10^{6} M_{\odot}\right), m_{*}=M_{*} / M_{\odot}$, and $r_{*}=R_{*} / R_{\odot}$. It is generally expected (Ulmer 1999; Strubbe \& Quataert 2009), although far from established, that after a 


\section{CHAPTER 1. INTRODUCTION}

few fallback times energy dissipation in shocks will circularize the returned tidal debris into an accretion disk, which can begin transporting gas to the SMBH through viscous processes. The exact nature of the circularization mechanism is quite uncertain, because of the inherent difficulty in numerically simulating thin debris streams travelling between a pericenter $\sim 10 R_{\mathrm{g}}$ and an apocenter $\sim 10^{4} R_{\mathrm{g}}$.

Although we discuss the complex details of disk formation in greater detail in Chapter 1.4 and Chapter 5, we will skip ahead here to discuss basic properties of the disk, assuming it has been established. The viscous timescale at a radius $R$ in the disk will be

$$
t_{\mathrm{visc}}=\alpha^{-1} \Omega^{-1}(R)\left(\frac{H}{R}\right)^{-2},
$$

which is generally much shorter than both the mass fallback timescale and the time $t$ since tidal disruption. Here $\alpha<1$ is the dimensionless Shakura-Sunyaev viscosity parameter (Shakura \& Sunyaev 1973), $H(R)$ is the disk scale height, and $\Omega(R)$ is the orbital frequency. Material that returns to the disk is quickly accreted onto the SMBH, leading to a roughly steady-state accretion flow. The rate at which this accretion flow is fed with mass will be $\dot{M}=(\mathrm{d} M / \mathrm{d} \epsilon)(\mathrm{d} \epsilon / \mathrm{d} t)$. For Keplerian orbits, the derivative of orbital energy with respect to orbital period is $\mathrm{d} \epsilon / \mathrm{d} t \propto t^{-5 / 3}$ (Phinney 1989). If we now make the assumption, valid at late times, that the distribution of stellar debris mass $\mathrm{d} M / \mathrm{d} \epsilon$ is roughly flat with orbital specific energy, then

$$
\dot{M}=\frac{M_{*}}{3 t_{\text {fall }}}\left(\frac{t}{t_{\text {fall }}}\right)^{-5 / 3} .
$$

This equation is often taken as evidence that the luminosity of a TDE flare should decline as $t^{-5 / 3}$; however, this is only true for the bolometric luminosity (Lodato \& Rossi 2011), and is only valid at late times. At early times, our assumption of constant $\mathrm{d} M / \mathrm{d} \epsilon$ 


\section{CHAPTER 1. INTRODUCTION}

is not valid and the original structure of the disrupted star is imprinted onto the light curve evolution (Lodato et al. 2009; Guillochon \& Ramirez-Ruiz 2013). For most SMBHs below the Hills mass, this rate of mass accretion is initially highly super-Eddington. If we assume a radiative efficiency $0<\eta<1$, the Eddington-limited mass fallback rate

$$
\dot{M}_{\text {Edd }}=1.37 \times 10^{21} \mathrm{~kg} \mathrm{~s}^{-1} \eta_{-1}^{-1} M_{6}
$$

and the peak rate of mass fallback (i.e. mass fallback at $t=t_{\text {fall }}$ ) is

$$
\frac{\dot{M}_{\text {peak }}}{\dot{M}_{\text {Edd }}}=133 \eta_{-1} M_{6}^{-3 / 2} m_{*}^{2} r_{*}^{-3 / 2} .
$$

Here we have defined $\eta_{-1}=\eta / 0.1$. While super-Eddington, the thick, radiation pressure-dominated accretion flow may drive a powerful outflow capable of powering an exceptionally bright electromagnetic transient (Strubbe \& Quataert 2009, 2011); we elaborate on this possibility in Chapter 1.5. The fallback rate will decline below the Eddington limit at a time

$$
t_{\text {Edd }}=2.1 \text { yr } \eta_{-1}^{3 / 5} M_{6}^{-2 / 5} m_{*}^{1 / 5} r_{*}^{3 / 5},
$$

after which the accretion disk will settle into a cooler and geometrically thinner configuration (Ulmer 1999). The timescales we have presented here are compared in Fig. 1.2.

\subsubsection{The Role of General Relativity}

Because real black holes are highly relativistic objects, the simple Newtonian picture presented above will be complicated by GR effects. Indeed, the first mentions of stellar tidal disruption in the literature were made by relativists interested in exotic ways to activate the classical Penrose process (Wheeler 1971). Although this original motivation 


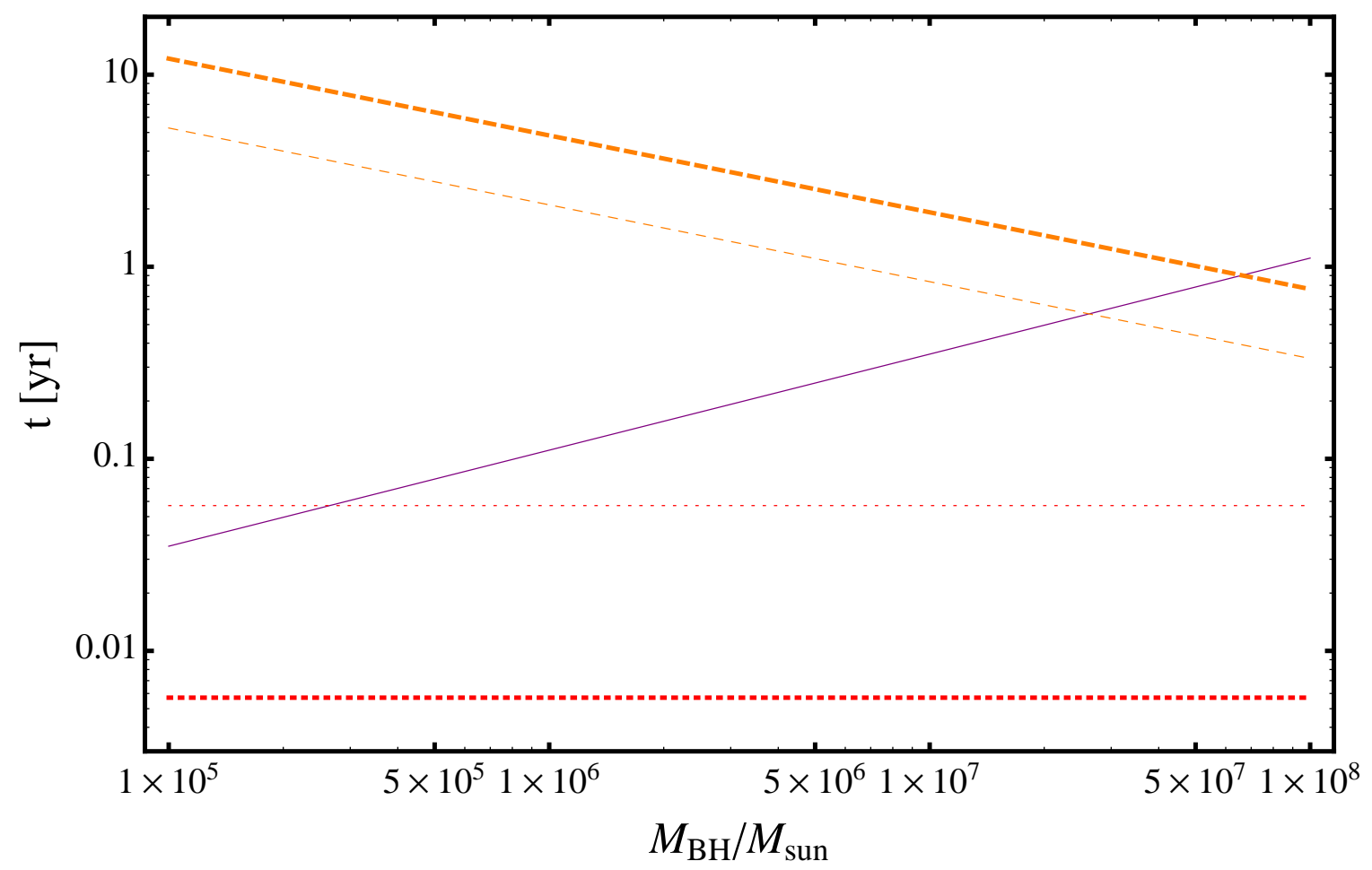

Figure 1.2.-: Timescales relevant for the disruption of a solar type star by a SMBH. The solid purple line is $t_{\text {fall }}$ as given by Eq. (1.6). The red dotted lines are the viscous timescale, Eq. (1.7) at an initial disk outer edge of $2 R_{\mathrm{t}}$ and assuming $H / R=0.5$; the thick line assumes $\alpha=0.1$ and the thin line assumes $\alpha=0.01$. The dashed orange lines represent the Eddington timescale, Eq. (1.11), for radiatively efficient accretion onto Schwarzschild $(\eta=0.1$, thin curve) and extremal Kerr $(\eta=0.4$, thick curve) SMBHs. Early time accretion fails to be super-Eddington for $M_{\mathrm{BH}} \gtrsim$ few $\times 10^{7} M_{\odot}$. Matter circularized into the disk will generally accrete rapidly $\left(t_{\text {fall }} \gg t_{\text {visc }}\right)$, although this assumption can break down for very small $M_{\mathrm{BH}}$ and $\alpha$. 


\section{CHAPTER 1. INTRODUCTION}

no longer seems realistic, GR effects still lead to qualitatively new behavior that can be studied by TDE observations.

One simple effect concerns whether stars can be tidally disrupted or not. The Hills mass is an approximate limit in two ways, both involving the neglect of general relativity (GR) in Eq. (1.4). First, most astrophysical TDEs are expected to arise from zero energy, parabolic orbits. In GR, the minimum pericenter distance for such an orbit overlaps with the innermost bound circular orbit, or IBCO (Bardeen et al. 1972). For a non-spinning $\mathrm{SMBH}$, the IBCO is located at $4 R_{\mathrm{g}}$, where $R_{\mathrm{g}}=G M_{\mathrm{BH}} / c^{2}=2 R_{\mathrm{s}}$. So for non-spinning black holes, a more accurate Hills mass would have a prefactor of $4.0 \times 10^{7} M_{\odot}$. However, SMBH spin (in dimensionless units, a) can reposition the IBCO between $1 R_{\mathrm{g}}$ (prograde equatorial orbits, $a=1$ ) and $5.83 R_{\mathrm{g}}$ (retrograde equatorial orbits, $a=-1$ ). The IBCO is also angle-dependent, and for prograde (retrograde) orbits will increase (decrease) as the orbit becomes increasingly inclined. Favorable combinations of SMBH spin and stellar angle-of-incidence can lead to observable TDE flares for SMBH masses an order of magnitude larger than Eq. (1.4), as was shown in Beloborodov et al. (1992) and Kesden (2012b).

General relativistic gravity can also have more subtle implications. In Newtonian gravity, the vertical collapse of the star is governed by self-similar equations (Carter \& Luminet 1983); this is not the case in GR (Luminet \& Marck 1985, also Chapters 1.3 and 4), which can lead to multiple compressions and bounces for high- $\beta$ orbits. Circularization of TDE debris streams may be aided by apsidal GR precession, or hindered by nodal GR precession (Kochanek 1994); these possibilities are explored in greater detail in Chapters 1.4 and Chapter 5. Finally, Lense-Thirring torques felt by the circularized accretion disk can induce near rigid body precession, as is described in 
Chapter 6.

\subsection{Tidal Disruption Event Rates}

\subsubsection{Two Body Relaxation}

Most stars in a galaxy travel on effectively collisionless orbits, with trajectories determined by the smooth, large-scale potential of the galaxy rather than by discrete interactions with other stars. If we view a population of stars as a fluid out of thermodynamic equilibrium, the collisionless Boltzmann equation is generally an accurate approximation, with which the dynamics of isolated or interacting galaxies can be studied. Quantitative justification can be found for these claims by calculating, to order of magnitude, the two-body relaxation timescale for orbits in a uniform density stellar environment (Binney \& Tremaine 2008, Eq. 1.38):

$$
t_{\mathrm{r}} \sim 0.1 \frac{N}{\ln N} t_{\text {orb }} .
$$

Here $N$ is the number of stars in the system, and $t_{\text {orb }}$ is the orbital timescale. For a typical galaxy, $N \sim 10^{11}$ and $t_{\text {orb }} \gtrsim 10^{6}$ yr, so $t_{\mathrm{r}} \gg t_{\text {Hubble: }}$ the galaxy is effectively collisionless.

However, the assumptions of a smooth background potential and collisionless orbits can break down in regions of extreme stellar density, such as globular clusters and galactic

nuclei. For many of these dense stellar systems, $t_{\mathrm{r}} \lesssim t_{\text {Hubble }}$, so two-body interactions relax stellar distribution functions toward equilibria within a Hubble time. We refer to such dense stellar systems as "collisional," even though physical collisions between 


\section{CHAPTER 1. INTRODUCTION}

stars are generally rare; our terminology refers primarily to close gravitational two-body encounters that alter stellar orbits. This type of relaxation is a crucial component of TDE rate calculations.

In the vicinity of a SMBH, the population of stars can be treated as a distribution function $f(\vec{x}, \vec{v})$ over a six dimensional phase space. For now, we only consider spherically symmetric stellar systems, meaning that these phase space coordinates map to a conserved specific energy and angular momentum, i.e. $f(\epsilon, J)$. This distribution function will be depleted in a zone of phase space known as the "loss cone"," defined in terms of specific angular momentum as

$$
J_{\mathrm{LC}}^{2}(\epsilon) \approx 2 G M_{\mathrm{BH}} R_{\mathrm{t}}
$$

As in prior sections, $M_{\mathrm{BH}}$ is the black hole mass and $R_{\mathrm{t}}$ the stellar radius (we will specialize for now to the idealized case of single-mass stellar populations). Any star with specific orbital angular momentum $J=|\vec{x} \times \vec{v}|<J_{\text {LC }}$ will be tidally disrupted in an orbital timescale, as its pericenter $R_{\mathrm{p}}<R_{\mathrm{t}}$. In a purely collisionless stellar system, the loss cone will drain on dynamical timescales and leave a permanent scar on $f(\vec{x}, \vec{v})$. However, in a collisional stellar environment, the relaxation of stars through $\epsilon$ and $J$ space will slowly refill the loss cone (Bahcall \& Wolf 1976; Frank \& Rees 1976; Lightman \& Shapiro 1977; Cohn \& Kulsrud 1978). For the highly eccentric orbits which produce most TDEs, the angular momentum relaxation timescale $t_{\mathrm{J}}$ is much less than the energy relaxation timescale, $t_{\mathrm{r}}$. Specifically, the time it takes for $J$ to change by its initial value

\footnotetext{
${ }^{4}$ This terminology appears to have been taken by analogy from plasma kinetic theory in the 1970s (Cohn \& Kulsrud 1978). At the time, American fusion research focused on magnetic confinement devices known as magnetic mirrors, which eventually proved nonviable due to plasma leaks from a geometrically conical region of phase space.
} 


\section{CHAPTER 1. INTRODUCTION}

is

$$
t_{\mathrm{J}}(\epsilon, e)=t_{\mathrm{r}}(\epsilon) \frac{J^{2}}{J_{\mathrm{c}}^{2}(\epsilon)} \approx t_{\mathrm{r}}(\epsilon)(1-e)^{2} .
$$

Here $J_{\mathrm{c}}(\epsilon)$ is the angular momentum of a circular orbit at fixed energy. Likewise, $e$ is the orbital eccentricity at fixed semimajor axis $a(\epsilon)$, in the limit of the almost Keplerian orbits where $a(\epsilon)<r_{\text {infl }}$. We have defined the SMBH's radius of influence, $r_{\text {infl }}$, to be the radius which contains twice the black hole's mass in stars.

The relaxational repopulation of loss cone orbits occurs in two different regimes (Frank \& Rees 1976; Lightman \& Shapiro 1977). Close to the SMBH, where the loss cone is much larger than typical collisional perturbations $\left(\Delta J \ll J_{\mathrm{LC}}\right)$, stars slowly diffuse into the loss cone across many orbital periods. In this "diffusive" regime, almost all TDEs have $\beta \approx 1$. Far from the $\mathrm{SMBH}, \Delta J \gg J_{\mathrm{LC}}$, and a star can safely wander into and out of the loss cone many times during a single orbit. In this "pinhole" regime, stars are tidally disrupted with a large range of $\beta$ values. The two regimes can be differentiated with a parameter,

$$
q(\epsilon)=\frac{\Delta J^{2}(\epsilon)}{J_{\mathrm{LC}}^{2}(\epsilon)}=\frac{P(\epsilon)}{t_{\mathrm{LC}}(\epsilon)},
$$

where $P(\epsilon)$ is the orbital period, and $t_{\mathrm{LC}}(\epsilon)=t_{\mathrm{r}}(\epsilon)\left(R_{\mathrm{t}} / a(\epsilon)\right)^{2}$ is the characteristic time it takes for $J$ to change by $J_{\mathrm{LC}}$. The diffusive regime occurs for $q(\epsilon) \ll 1$; the pinhole regime for $q(\epsilon) \gg 1$.

Although the idealized theory of collisional stellar dynamics near loss cones was developed long ago, it was only more recently that observations of nearby galactic nuclei enabled realistic TDE rates to be calculated (Magorrian \& Tremaine 1999; Wang \& Merritt 2004). For most observed galaxies, the net flux of stars into the loss cone peaks near a radius $r_{\text {crit }}$, which is defined as the radius where a circular orbit has $q\left(\epsilon_{\text {crit }}\right)=1$. 


\section{CHAPTER 1. INTRODUCTION}

This critical radius, from where most TDEs originate, is by coincidence $\sim r_{\text {infl }}$ (Wang \& Merritt 2004). Therefore most TDEs arise from orbits at $\sim 10^{0}-10^{1}$ pc scales, validating Chapter 1.2's approximation of parabolic trajectories.

Using empirically constructed galaxy models based on Hubble Space Telescope (HST) observations by the Nuker team (Lauer et al. 1995), both Magorrian \& Tremaine (1999) and Wang \& Merritt (2004) have estimated TDE rates over a sample of nearby

galaxies. Both of these papers find TDE rates $10^{-4} \mathrm{yr}^{-1} \lesssim \dot{N}_{\text {TDE }} \lesssim 10^{-6} \mathrm{yr}^{-1}$, with the integrated rate dominated by low mass galaxies that contain a steep, power law stellar density profile. TDE rates in large galaxies are suppressed by the shallower ("cored") stellar density profiles, and also by the Hills mass limit. Both of these works may slightly underestimate the true TDE rate; Magorrian \& Tremaine (1999) systematically overestimates SMBH masses, while Wang \& Merritt (2004) ignores the enhancement to $\dot{N}_{\text {TDE }}$ from axisymmetric stellar potentials (which we elaborate on in the next sections).

\subsubsection{Resonant Relaxation}

Well inside a SMBH's radius of influence, the central potential of the black hole is so dominant that subrelativistic stellar orbits trace nearly closed Keplerian ellipses. If we time-average these orbits over many orbital periods, we can think of a stellar population as a system of mass-weighted wires exerting torques on each other (Rauch \& Tremaine 1996). The Poisson noise produced by the discreteness of the stellar distribution function $f(\vec{x}, \vec{v})$ leads to each wire feeling net torques from the larger population of wires. Over a "coherence timescale," $t_{\mathrm{coh}}$, these torques are coherent rather than stochastic, and can therefore lead to rapid changes in orbital parameters (unlike the uncorrelated random 


\section{CHAPTER 1. INTRODUCTION}

walk through phase space produced by two-body relaxation). The relevant $t_{\text {coh }}$ is the time it takes for the system of wires to change appreciably. Because the wires are fixed in space over a coherence timescale, the system's potential is stationary and orbital energy (therefore semimajor axis) is constant for each star. However, other orbital parameters are not.

Coherent changes in an orbit's eccentricity, $e$, are referred to as "scalar resonant relaxation" (SRR); coherent changes in the orbit's orientation, $\hat{J}$, are referred to as "vector resonant relaxation" (VRR). The VRR timescale is generally orders of magnitude shorter than the SRR timescale (Hopman \& Alexander 2006). Both SRR and VRR lead to rapid evolution of $\vec{J}$ for timescales $t<t_{\text {coh }}$, but random walks (in $e$ and $\hat{J}$, respectively) for $t>t_{\text {coh }}$. Orbits at fixed semimajor axis $a$ experience retrograde "mass precession" from the background stellar potential on a timescale

$$
t_{\mathrm{m}} \sim \frac{M_{\mathrm{BH}}}{N(<a) M_{*}} P(a)
$$

where $N(<a)$ is the number of stars at smaller semimajor axis. For large values of $J$, this is the dominant source of precession, and $t_{\mathrm{coh}} \approx t_{\mathrm{m}}$. Closer to the black hole, GR apsidal precession dominates and $t_{\mathrm{coh}} \approx t_{\mathrm{GR}}$, where

$$
t_{\mathrm{GR}}=\frac{8}{3} \frac{J^{2}}{J_{\mathrm{IBCO}}^{2}} P(a),
$$

and $J_{\mathrm{IBCO}}=4 G M_{\mathrm{BH}} / c$ is the Newtonian angular momentum of an IBCO-grazing parabolic orbit.

The dynamics of SRR change dramatically for pericenters very close to the SMBH, where GR torques have more subtle effects than simple reductions of the coherence time. Recent work has identified a "Schwarzschild barrier" in $\{a, e\}$ space, along the locus of orbital parameters where the SRR timescale $t_{\mathrm{SRR}}=t_{\mathrm{GR}}$. Contrary to some 


\section{CHAPTER 1. INTRODUCTION}

previous expectations, orbits do not simply accumulate at this barrier but instead bounce backwards to lower eccentricity states. This greatly reduces SRR's ability to produce high-eccentricity encounters between stellar mass objects and an SMBH (Merritt et al. 2011; Brem et al. 2012).

Even prior to the discovery of the Schwarzschild barrier, detailed calculations had found that resonant relaxation would be a subdominant source of TDEs relative to standard two-body relaxation (Rauch \& Tremaine 1996; Rauch \& Ingalls 1998; Hopman \& Alexander 2006; Madigan et al. 2011). This is because the bulk of stellar flux into the loss cone comes from scales $r_{\text {crit }} \sim r_{\text {infl }}$ at which resonant relaxation is ineffective due to mass precession from the stellar potential. However, SRR was once thought to dominate the rate of "extreme mass ratio inspirals" (EMRIs). In an EMRI, a compact stellar mass object spirals into an SMBH under gravitational radiation reaction. If observed by a LISA-like instrument, EMRIs would serve as superb tracers of the Kerr spacetime, enabling precision tests of GR by measuring SMBH multipole moments (Sopuerta 2010). An important side motivation for studying TDEs is to better understand the nuclear stellar populations and relaxation processes that generate EMRIs. However, the existence of the Schwarzschild barrier severely reduces realistic EMRI rates (Merritt et al. 2011) $)^{5}$, and also reduces the contribution of SRR to the TDE rate even further. It is therefore reasonable to disregard SRR in TDE rate calculations, except in unusual situations where two-body relaxation is suppressed or a large population of stars exists

\footnotetext{
${ }^{5}$ It has recently been argued (Brem et al. 2012) that a subpopulation of very high eccentricity EMRIs may successfully penetrate the barrier; these were previously disregarded because it was thought they would plunge directly into the horizon. Properly accounting for SMBH spin shows that many of these "plunge" EMRIs can in fact accumulate high SNR in the LISA band (Amaro-Seoane et al. 2013).
} 
CHAPTER 1. INTRODUCTION

at very small semimajor axis.

\subsubsection{Alternative Relaxational Mechanisms}

Beyond two-body and resonant relaxation in spherical potentials, other mechanisms can also feed stars to SMBHs. In particular, axisymmetric or triaxial stellar potentials, infalling massive perturbers, SMBHBs, and the tidal separation of stellar binaries have all been suggested as ways to enhance a galaxy's TDE rate. We will briefly review each of these mechanisms here.

A significant enhancement to the TDE rate can arise if the central distribution of stars is axisymmetric or triaxial. Orbits in nonspherical potentials generally fail to conserve all components of angular momentum, and orbits in fully triaxial potentials generally conserve no components of angular momentum. Over many orbital times, this lets an individual orbit sample pericenters of arbitrarily low value, eventually falling inside the tidal radius and causing a TDE. Axisymmetric potentials can lead to modest, factors of a few enhancements to the TDE rate (Magorrian \& Tremaine 1999; Vasiliev \& Merritt 2013). Orbits in these potentials still conserve the component of angular momentum parallel to the symmetry axis, but in a fully triaxial potential no component of $\vec{J}$ is conserved (Poon \& Merritt 2004), and a large subpopulation of stars lives on centrophilic, chaotic orbits. In such a potential, $\dot{N}_{\text {TDE }}$ can be enhanced by a factor $\sim 10$ relative to the two-body relaxation baseline (Merritt \& Poon 2004). Interestingly, the enhancement is greatest for large SMBHs near the Hills limit, which can achieve rates up to $\dot{N}_{\mathrm{TDE}} \sim 10^{-3} \mathrm{yr}^{-1}$. However, the long-term stability of these potentials remains unclear; in many cases, triaxial potentials evolve toward axisymmetry, reducing the TDE 


\section{CHAPTER 1. INTRODUCTION}

rate enhancement (Poon \& Merritt 2004). Due to a lack of observational data, it is unclear whether the stable triaxial configurations form naturally in galaxy evolution.

Periodically, the orbits of stars in a galactic nucleus can be perturbed by an infalling massive object - a giant molecular cloud, or a star cluster, for example. These perturbations will scatter stars through phase space, helping to fill the inner regions of the loss cone and enhancing the TDE rate. This enhancement has been estimated to be substantial, and can contribute at leading order to the total TDE rate. However, because these massive perturbers are unlikely to penetrate inward of $r_{\text {crit }}$ without themselves being tidally disrupted, their enhancement to the two-body $\dot{N}_{\text {TDE }}$ is at most a factor of a few (Perets et al. 2007).

A massive perturber can have a greater effect on the TDE rate if it penetrates well into the empty or diffusive loss cone regime. The hardening of a SMBHB, or SMBH-IMBH binary, can accomplish this. At orbital separations comparable to the larger black hole's influence radius, the combination of Kozai effects (Ivanov et al. 2005) and chaotic three-body interactions (Chen et al. 2009, 2011) can lead to a huge enhancement in the TDE rate, perhaps up to $\sim 10^{-1}$ disruptions per year. This phase is short-lived, however, typically lasting $\sim 10^{5} \mathrm{yr}$ and ending once ejection and disruption of the available stars has led the SMBH binary system to stall its hardening (Chen et al. 2011). Nonetheless, the total number of stars disrupted is substantial, and TDEs generated by SMBHBs may account for up to $\sim 10 \%$ of the total TDE rate (Chen et al. 2011; Wegg \& Nate Bode 2011). Following the merger of an SMBHB, GW recoil will tilt the loss cone in phase space, instantaneously refilling a portion of it; this can also elevate the TDE rate to $\sim 10^{-1}$ yr (Stone \& Loeb 2011) and will be discussed in greater detail in Chapter 2. 


\section{CHAPTER 1. INTRODUCTION}

A final source of stars to be disrupted arises when a SMBH tidally separates a binary star system (Hills 1988). One star is ejected at high speeds, often to become a hypervelocity star. The other becomes bound to the SMBH, and its subsequent orbital evolution (driven by a combination of two-body relaxation, SRR, and GW emission) determines whether it will be tidally disrupted (Amaro-Seoane et al. 2012a). If GW emission dominates, the bound star will circularize efficiently and begin stably transferring mass to the SMBH at a low luminosity (Dai \& Blandford 2011). Although such mass transfer is interesting and potentially detectable, it differs qualitatively from a violent TDE. If two-body angular momentum relaxation or SRR is able to drive $R_{\mathrm{p}}<R_{\mathrm{t}}$, a TDE will be produced; however, the discovery of the Schwarzschild barrier has made this scenario somewhat uncertain. Nonetheless, the production rate of bound stars from binary separation is so large that if a substantial fraction can evolve to low angular momentum orbits, they could dominate the TDE rate and perhaps contribute substantially to SMBH growth (Bromley et al. 2012). TDEs originating from this channel might visibly differ from standard TDEs due to their lower eccentricity, which would manifest in a nonstandard light curve (Hayasaki et al. 2012, see also Chapter 5).

\subsection{Dynamics of Disruption}

The pioneering work of Carter \& Luminet (1983) introduced a shorthand for the dynamical phases of strong star-SMBH tidal encounters which we adapt for our purposes in this paper. In phase $\mathrm{I}$, the star is still a distance $R>R_{\mathrm{t}}$ from the $\mathrm{SMBH}$, and exists in a slightly perturbed hydrostatic equilibrium. During this phase, the weak tidal forces from the SMBH begin to excite oscillatory modes in the star, which can be 
described with a straightforward linear multipole formalism (Press \& Teukolsky 1977).

For nondisruptive tidal encounters $(\beta \lesssim 1)$, the star survives its first pericenter passage, although some mass may be lost from its envelope. Repeated pericenter passages likely occur if the star is in the "diffusive" loss cone regime, and can lead to continued mode excitation. On longer timescales the dissipation of mode energy can lead to thermal expansion of the star's envelope (Novikov et al. 1992), modulations of stellar luminosity (Alexander \& Morris 2003), and runaway tidal stripping of the star (Li \& Loeb 2013).

\subsubsection{Vertical Collapse and Crunch: (Semi-)Analytic}

Alternatively, if $\beta \gtrsim 1$, a violent and fully disruptive TDE will ensue. In phase II of a TDE, $R<R_{\mathrm{t}}$ and the star's internal forces are subdominant to the tidal acceleration it experiences. During this stage of stellar disruption, especially if $\beta$ is large, the star undergoes approximate tidal free fall, with its fluid elements moving on roughly ballistic or geodesic trajectories (altered slightly by the subdominant internal forces). It is in this regime where the dynamics of the tidal tensor,

$$
\ddot{r}_{i}=C_{i j} r_{j}
$$

are most relevant. In this notation, the vector components $r_{i}$ represent positions of stellar fluid elements in the star's center of mass frame. The Newtonian tidal tensor (Brassart \& Luminet 2008) is given by

$$
C_{i j}^{\mathrm{N}}=\frac{G M_{\mathrm{BH}}}{R^{3}}\left(-\delta_{i j}+3 \frac{R_{i} R_{j}}{R^{2}}\right) .
$$




\section{CHAPTER 1. INTRODUCTION}

Denoting $X=R \cos f, Y=R \sin f$, and the $Z$ direction (normal to the orbital plane) as the 1st, 2nd and 3rd principal axes, respectively, the nonzero components of $C_{i j}^{\mathrm{N}}$ are

$$
\begin{aligned}
C_{11}^{\mathrm{N}} & =-\frac{M_{\mathrm{BH}}}{R^{3}}\left(1-3 \cos ^{2} f\right) \\
C_{22}^{\mathrm{N}} & =-\frac{M_{\mathrm{BH}}}{R^{3}}\left(1-3 \sin ^{2} f\right) \\
C_{33}^{\mathrm{N}} & =-\frac{M_{\mathrm{BH}}}{R^{3}} \\
C_{12}^{\mathrm{N}} & =-\frac{M_{\mathrm{BH}}}{R^{3}}(-3 \cos f \sin f) .
\end{aligned}
$$

Likewise, the general relativistic tidal tensor in Schwarzschild spacetime $(G=c=1)$ is given by (Brassart \& Luminet 2010)

$$
\begin{aligned}
& C_{11}^{\mathrm{GR}}=-\frac{G M_{\mathrm{BH}}}{R^{3}}\left(1-3 \frac{R^{2}+L^{2}}{R^{2}} \cos ^{2} F\right) \\
& C_{22}^{\mathrm{GR}}=-\frac{G M_{\mathrm{BH}}}{R^{3}}\left(1-3 \frac{R^{2}+L^{2}}{R^{2}} \sin ^{2} F\right) \\
& C_{33}^{\mathrm{GR}}=-\frac{G M_{\mathrm{BH}}}{R^{3}}\left(1+3 \frac{L^{2}}{R^{2}}\right) \\
& C_{12}^{\mathrm{GR}}=-\frac{G M_{\mathrm{BH}}}{R^{3}}\left(-3 \frac{R^{2}+L^{2}}{R^{2}} \cos F \sin F\right)
\end{aligned}
$$

In both cases, $C_{i j}$ is a symmetric tensor, so $C_{21}=C_{12}$. The Schwarzschild case differs from the Newtonian one in two notable ways: tidal accelerations are increased by the addition of terms $\sim L^{2} / R^{5}$, and we have used a slightly different orbital anomaly, replacing the Newtonian $f$ with a variable $F$ that equals 0 at pericenter and evolves as $\dot{F}=E L /\left(R^{2}+L^{2}\right)$. Here $E$ and $L$ are the GR specific orbital energy and angular momentum, respectively. The full Kerr metric tidal tensor, relevant for spinning SMBHs, is beyond the scope of this thesis, but is provided in Marck (1983a,b); Kesden (2012b). The most important qualitative difference between the Newtonian and GR tidal tensors concerns the vertical, $C_{33}$ component: in the Newtonian case, $\ddot{z} \propto z$, leading to a roughly homologous collapse (perfectly homologous if internal forces are neglected). Vertical 


\section{CHAPTER 1. INTRODUCTION}

tidal compression in general relativistic gravity is stronger and not self-similar.

Phase II of a TDE comes to an end when the vertical compression of the star has adiabatically increased internal pressure to the point where it competes with and then dominates both tidal acceleration and the inertia of the star's vertical collapse. The resulting vertical "bounce" of the star defines the short-lived phase III of a TDE, where the vertical pressure gradient leads to a rapid reversal of the ongoing compression. For $\beta \approx 1$, phases II and III will be overlapping and ill-defined, because in a marginal disruption (i) the tides do not strongly compress the star, (ii) the star does not remain in the tidal sphere for long, and (iii) internal forces never become truly subdominant. Further complications to this simple picture arise from the fact that different portions of the star enter the tidal sphere at different times, so that the "leading edge" of the star collapses and bounces before the center; also, the existence of a strong density contrast between the mean stellar density and the stellar core will alter the picture of tidal free fall, as the core can only disrupt at a radius $R_{\mathrm{t}}^{\prime}<R_{\mathrm{t}}$ (Guillochon et al. 2009).

The complex dynamics of phases I-III of stellar tidal disruption intermingle orbital mechanics, hydrodynamics, GR, and in some cases even nuclear fusion reactions. These dynamics have been studied analytically, semi-analytically using the so-called "affine model," and with the use of direct numerical simulation, in either one or three dimensions. We now will quickly review the existing literature on these stages of a TDE.

The earliest analytic estimates of hydrodynamic effects in phases II and III date back to Carter \& Luminet (1982) and Carter \& Luminet (1983), who were initially interested in detonating tidally compressed stars through runaway thermonuclear reactions. By analyzing the vertical equation of motion for a tidally free falling column of star, i.e. Eqs. 
(1.18) and (1.22), these authors estimated that the external "piston" of tidal acceleration will compress the column until the star's internal energy reaches

$$
U_{\mathrm{c}} \sim \beta^{2} U_{*},
$$

the star's central density reaches

$$
\rho_{\mathrm{c}} \sim \beta^{-2 /(\gamma-1)} \rho_{*},
$$

and the star's central temperature reaches

$$
T_{\mathrm{c}} \sim \beta^{2} T_{*} .
$$

In these approximate equations, $U_{*}=G M_{*} / R_{*}, \rho_{*}$ is the pre-disruption central density of the star, $T_{*}$ is the pre-disruption central temperature of the star, and $\gamma$ is the star's adiabatic index. The duration of peak compression, i.e. phase III, will only last for a timescale

$$
\tau_{\mathrm{c}} \sim \beta^{-(\gamma+1) /(\gamma-1)} \tau_{*},
$$

where $\tau_{*}=\sqrt{R_{*}^{3} /\left(G M_{*}\right)}$. In Stone et al. (2012b), we coupled these hydrodynamic scaling relations to a more precise analytic model for tidal free fall to investigate the $\beta$ dependence of $\Delta \epsilon$; this is described in Chapter 4 .

Much early progress on the problem of stellar tidal disruption was made using the framework of the affine model (Carter \& Luminet 1983), which combines the tensor virial theorem with the assumption that a tidally disrupting star can be thought of as nested ellipsoidal shells. The affine model accounts for the SMBH tidal field, stellar self-gravity, and hydrodynamic forces, and can loosely be thought of as an extension of stellar perturbation theory (Press \& Teukolsky 1977) to nonlinear regimes, but restricted to the $\ell=2$ mode. The earliest work in the affine framework validated the approximate 


\section{CHAPTER 1. INTRODUCTION}

hydrodynamic scaling relations in Eqs. (1.28), (1.29), (1.30), and (1.31). Later papers calibrated these relations and surveyed more widely the joint parameter space of $\beta$ and $M_{*}$ (Luminet \& Carter 1986). Comparisons to full hydrodynamical simulations indicate that the affine approximation becomes much less applicable after phase III of a disruption, when the stellar matter deviates strongly from the ellipsoidal approximation. The affine model was later placed on a more formal footing (Carter \& Luminet 1985), and extended to include the effects of GR (Luminet \& Marck 1985) and thermonuclear reactions (Luminet \& Pichon 1989). The increased vertical tidal acceleration due to general relativistic gravity, i.e. Eq. (1.26), can lead to the star recollapsing and undergoing a second vertical bounce, provided its orbit is relativistic $\left(R_{\mathrm{p}} \sim R_{\mathrm{IBCO}}\right)$ and deeply penetrating $(\beta \gg 1)$. During single or multiple collapses of a main sequence star, the conditions for explosive helium burning are generally not satisfied, but alpha-capture and proton-capture reactions may lead to significant nucleosynthesis. Within the affine framework, these reactions change the composition of the star at the $\sim 1-5 \%$ level and for $\beta \gtrsim 10$ release many times the star's original binding energy, but are still energetically subdominant to the tidally imprinted spread of debris energy, $\Delta \epsilon$ (Luminet \& Pichon 1989).

More recent extensions of the affine model allow individual ellipsoidal shells to have different properties and orientations (Ivanov \& Novikov 2001), enabling a treatment of partial mass loss in encounters that are not fully disruptive $(\beta \lesssim 1)$. Later work using the extended affine model found reasonably good agreement between its results and those of three dimensional grid-based simulations (Ivanov et al. 2003). The extended affine model has also been used to produce realistic, angle-dependent cross-sections for disruption and swallowing of main sequence stars by spinning SMBHs (Ivanov \& Chernyakova 2006). 


\subsubsection{Vertical Collapse and Crunch: Hydrodynamic Simulations}

Although the affine model and its descendants are computationally efficient and provide analytic insight, they are ultimately limited by their assumptions, so for a fully self-consistent treatment of stellar tidal disruption it is necessary to use hydrodynamical simulations. Such simulations discretize either mass or volume and then integrate the equations of hydrodynamics in a Lagrangian or Eulerian way, respectively. For the problem of stellar TDEs, the Lagrangian approach is generally implemented with Smoothed Particle Hydrodynamics (Lucy 1977, hereafter SPH) algorithms, dating back to the pioneering work of Nolthenius \& Katz (1982), which was limited by the small number of SPH particles used per simulation (40). Subsequent simulations by Bicknell \& Gingold (1983) increased the number of SPH particles to 2000, and found significantly less tidal compression than in the affine model. This was later attributed to numerical viscosity and insufficient vertical resolution (Luminet \& Carter 1986), but as we shall see, disagreements along these lines have persisted with more modern simulations. Later generations of three dimensional SPH simulations verified analytic expectations for $\Delta \epsilon$ (Evans \& Kochanek 1989, but only for the $\beta=1$ case), examined the role of GR by adapting SPH to curved spacetimes (Laguna et al. 1993), followed the evolution of returning debris streams (Ayal et al. 2000), measured the imprint of stellar structure on the early-time, rising portion of a TDE lightcurve (Lodato et al. 2009), and simulated debris circularization in eccentric center of mass trajectories (Hayasaki et al. 2012, see also Chapter 5).

Complementary efforts by grid-based hydrodynamics codes began later, with early work focusing on partial mass loss and mode excitation in weak tidal encounters 


\section{CHAPTER 1. INTRODUCTION}

(Khokhlov et al. 1993b), as well as the transition to complete disruption for higher $\beta$ values (Khokhlov et al. 1993a). Similar techniques were combined with the GR tidal tensor and center of mass trajectories to examine the impact of relativity on disruptions of white dwarfs by IMBHs (Frolov et al. 1994). A subsequent paper conducted the first systematic exploration of parameter space for nondisruptive tidal encounters (Diener et al. 1997). More recently, grid-based one dimensional simulations have followed vertically collapsing columns of star at high resolution with the aim of precisely capturing shocks launched during phase III, both in Newtonian (Brassart \& Luminet 2008) and general relativistic (Brassart \& Luminet 2010) gravity. These one dimensional works found degrees of compression in close agreement with earlier affine model studies (Luminet \& Marck 1985; Luminet \& Carter 1986), but in disagreement with state of the art three dimensional Eulerian simulations (Guillochon et al. 2009), which found a significantly smaller degree of vertical compression. This difference is likely due to a combination of lower resolution in the three dimensional simulations, physical three dimensional effects (e.g., pressure waves communicating between the fully collapsed, phase III leading edge of the star, and the more central portions of the star still undergoing phase II compression), and possible inaccuracies introduced via one dimensional initial conditions ${ }^{6}$. A parameter study of three dimensional simulations has also investigated the $\beta$ dependence of the $\mathrm{d} M / \mathrm{d} \epsilon$ mass distribution, finding both the critical $\beta$ values leading to full disruption, and a lack of $\beta$ dependence in fully disruptive encounters (Guillochon \& Ramirez-Ruiz 2013). This last finding parallels analytic work (Stone et al. 2012b) presented in Chapter 4 of this thesis.

\footnotetext{
${ }^{6}$ James Guillochon, Morgan MacLeod, and Enrico Ramirez-Ruiz, private communication.
} 
CHAPTER 1. INTRODUCTION

\subsubsection{Debris Expansion and Circularization}

Following the vertical bounce of the star, internal forces again become negligible, beginning phase IV of a TDE: free expansion. At this point, half the star's mass is unbound from the SMBH and enters onto its escape trajectory, while the other half remains bound. In this phase of expansion, the star is governed by the same tidal equations that dominated phase II. As mentioned before, this can lead to another vertical collapse for deeply plunging, relativistic orbits. Eq. (1.18) ceases to describe the dynamics of the stellar debris as it leaves the tidal sphere, due to its increasing physical size and (for low $\beta$ ) the reemerging importance of internal forces. Phase V of a TDE the dynamics of thin, gaseous debris streams - is the least studied, due to the difficulty of direct numerical simulation. The most tightly bound debris stream has an eccentricity

$$
e_{\min }=1-\beta^{-1}\left(\frac{M_{\mathrm{BH}}}{M_{*}}\right)^{-1 / 3},
$$

implying that the orbital apocenter will be at least two orders of magnitude larger than orbital pericenter for star-SMBH TDEs. The evolution of the stellar debris as it leaves the tidal sphere depends sensitively on both $\beta$ and the initial structure of the star. For marginal, $\beta \approx 1$ disruptions, a surviving stellar core can accrete a large portion of the debris through gravitational collapse. Even for fully disruptive $\beta \approx 1$ encounters, self-gravity can have important effects on stream evolution (Kochanek 1994).

Two mechanisms for debris circularization have been proposed so far in the literature. In the first of these models, apsidal GR precession causes tightly bound debris that has completed its second pericenter passage to intersect with less bound debris still returning from its first apocenter. Shocks will form in the large-angle collision between these streams, dissipating energy and leading to circularization (Rees 1988), a process which 


\section{CHAPTER 1. INTRODUCTION}

has been seen in our hydrodynamical simulations of eccentric $(e=0.8)$ TDEs around SMBHs (Hayasaki et al. 2012, also see Chapter 5). This circularization mechanism could be delayed, perhaps strongly, by nodal GR precession from Lense-Thirring torques around rapidly spinning SMBHs (Kochanek 1994). An alternate type of circularization can arise from vertical compression of stellar debris during second (and later) pericenter passages, when the thin streams are funneled by tidal acceleration into a vertically compressed "nozzle." The convergent flow at pericenter leads to shocks. Direct energy dissipation in the nozzle is unlikely to be an efficient circularizer; if we assume roughly ballistic motion for the stellar debris between the first and second pericenter passages, the specific kinetic energy of vertical collapse at pericenter return will be $\epsilon_{\mathrm{z}} \sim \beta^{2}\left(G M_{*} / R_{*}\right)$, so that even if $100 \%$ of this energy is dissipated in shocks, the gas will need to return to pericenter

$$
N_{\text {peri }} \sim \frac{1}{2 \beta}\left(\frac{M_{\mathrm{BH}}}{M_{*}}\right)^{2 / 3}
$$

times in order to circularize ${ }^{7}$. However, shocks in the nozzle do change the orbital parameters of the gas exiting pericenter, which can lead to an effective apsidal precession, circularizing the gas through collisions and shocks with material returning from its first apocenter passage (as in the GR precession case). This type of circularization has been seen in simulations of stars on parabolic orbits disrupted by $10^{3} M_{\odot}$ IMBHs (Ramirez-Ruiz \& Rosswog 2009), but it is unclear whether this mechanism scales up

\footnotetext{
${ }^{7}$ This argument could break down if a large fraction of the debris re-collapses due to gravitational instability, but it is not clear whether that would aid or hinder nozzle-driven circularization (it would certainly hinder GR-driven circularization, by reducing the stream cross-section and magnifying the impact of nodal GR precession). This is because the collapsed streams will see a higher effective $\beta$, but will likely have a lower specific internal energy than the original star.
} 
CHAPTER 1. INTRODUCTION

to $e=1$, star-SMBH disruptions ${ }^{8}$. Analogously, the GR-mediated circularization seen in the $e=0.8$, star-SMBH TDEs of Hayasaki et al. (2012) has not been demonstrated to scale up to the canonical $e=1$, star-SMBH TDE scenario. The question of debris circularization is a subtle one that needs to be resolved before theorists can hope to understand the earliest emission from stellar tidal disruption flares. This is an important question to address because the early-time, rising portion of a TDE light curve may encode the parameters of the disrupted star (Lodato et al. 2009; Guillochon \& Ramirez-Ruiz 2013). A second motivation for understanding debris circularization is that if GR precession is the dominant mechanism, the spin of the SMBH may be encoded into the circularization timescale.

\subsection{Accretion of Circularized Debris}

Although the process of debris circularization is complex and, as of now, poorly understood, it is reasonable to assume that at some point after tidal disruption, a circularized accretion flow will exist around the SMBH. The evolution and emission of this transient accretion disk has been the subject of much theoretical study, as it relates much more directly to observations than do many of the subtle dynamical processes in

\footnotetext{
${ }^{8}$ The SPH simulations of Ayal et al. (2000) appear to capture nozzle-driven circularization in $e=1$, $M_{\mathrm{BH}}=10^{6} M_{\odot}$ TDEs. However, the reliability of these results may be limited by (i) the difficulty of accurately capturing shocks in SPH codes, (ii) the low particle resolution $(N=5000)$ of these simulations, and (iii) the particle-splitting algorithm employed to address resolution issues, since the vertical compression of the star will be sensitive to spurious changes in stream geometry. If vertical compression at pericenter is under-resolved, the net effect will be unphysical velocity perturbations in the orbital plane (Guillochon et al. 2009; Stone et al. 2012b) and correspondingly unphysical apsidal precession.
} 
Chapter 1.3 and Chapter 1.4.

The short viscous timescales of these disks suggest that mass accretion onto the SMBH is mediated by the supply of fallback material into the disk, rather than internal viscous processes. For example, in the simple analytic slim disk model of Strubbe \& Quataert (2009), the time since disruption is greater than Eq. (1.7) for $\sim 1-3$ yr. On timescales longer than this, a reservoir of fallback material will accumulate, and the supply of fuel to the SMBH will be governed by the (now slow) viscous transport of material within the accretion disk (Cannizzo et al. 1990). For SMBHs with $M_{\mathrm{BH}} \lesssim 2 \times 10^{7} M_{\odot}$, the disk will initially pass through a stage of super-Eddington accretion, before the mass fallback rate declines and the disk becomes cool and geometrically thin. At very late times, the disk may once again become thick as it transitions into a radiatively inefficient mode of accretion.

While the late-time evolution of the disk has been described analytically by Cannizzo et al. (1990), the early-time disk evolution can be studied with the time-dependent slim disk model of Strubbe \& Quataert (2009). In this model, the disk can be treated as a multicolor blackbody, where each disk mass annulus of radius $R$ possesses an effective temperature

$$
T_{\text {eff }}^{4}=\frac{3 G M_{\mathrm{BH}} \dot{M} f}{8 \pi \sigma R^{3}}\left\{\frac{1}{2}+\left[\frac{1}{4}+\frac{3}{2} f\left(\frac{10 \dot{M}}{\dot{M}_{\mathrm{Edd}}}\right)^{2}\left(\frac{R}{R_{\mathrm{S}}}\right)^{-2}\right]^{1 / 2}\right\}^{-1},
$$

and scale height

$$
\frac{H}{R}=\frac{3 f}{4} \frac{10 \dot{M}}{\dot{M}_{\text {Edd }}} \frac{R_{\mathrm{S}}}{R}\left\{\frac{1}{2}+\left[\frac{1}{4}+\frac{3}{2} f\left(\frac{10 \dot{M}}{\dot{M}_{\text {Edd }}}\right)^{2}\left(\frac{R}{R_{\mathrm{S}}}\right)^{-2}\right]^{1 / 2}\right\}^{-1} .
$$

Here $\sigma$ is the Stefan-Boltzmann constant, and $f=1-\left(R_{\mathrm{ISCO}} / R\right)^{1 / 2}$. Because of the simplicity of this model, we will make use of it several times in this paper (Chapters 3, 


\section{CHAPTER 1. INTRODUCTION}

$6)$.

Although the bolometric luminosity of TDE accretion disks (at early times, when $\left.t<t_{\text {visc }}\right)$ should scale as $L \propto \dot{M} \propto t^{-5 / 3}$, disk emission is likely peaked in the UV or soft X-ray, and wavelengths far from this peak will see distinct time evolution. In particular, if we are observing the Rayleigh-Jeans tail of disk emission (as will usually be the case for optical instruments), $L \propto t^{-5 / 12}$ (Lodato \& Rossi 2011). At very late times, observations may move to wavelengths shorter than the blackbody temperature of the inner disk edge; at this point the observed luminosity will exponentially decline in time. It remains unclear why so many UV and optical observations of TDEs see luminosity declining with approximately $t^{-5 / 3}$ power laws (Gezari et al. 2012, for example); these observations may suggest significant reprocessing of disk luminosity.

At the low end of the SMBH mass function, a significant source of non-continuum emission may be emission lines formed when the unbound debris reprocesses hard radiation from the central accretion disk. The half of the star that is dynamically ejected expands as it travels away from the $\mathrm{SMBH}$, forming a wall of gas subtending a solid angle

$$
\Delta \Omega \sim 48^{1 / 2}\left(R_{*} / R_{\mathrm{t}}\right)^{3 / 2} \sim 7 \times 10^{-3} M_{6}^{-1 / 2} m_{*}^{1 / 2}
$$

on the sky (Strubbe \& Quataert 2009). The gas remains mostly neutral, but with a very thin ionization front that emits broad emission lines (the velocity dispersion of the gas is $\left.\Delta v \approx \sqrt{\Delta \epsilon} \approx 4.4 \times 10^{6} \mathrm{~m} \mathrm{~s}^{-1} M_{6}^{1 / 2} m_{*}^{1 / 3} r_{*}^{-1 / 2}\right)$. These lines are generally weak, but are stronger for small SMBHs and at late times (Strubbe \& Quataert 2009). Recombination in the ejected gas may also power a short-lived optical transient (Kasen \& Ramirez-Ruiz 2010). 


\section{CHAPTER 1. INTRODUCTION}

During the earliest stages of disk accretion, when $t<t_{\text {Edd }}$, the accretion disk will be geometrically thick and radiation pressure dominated. There are many uncertainties about such super-Eddington accretion flows, but most existing radiation hydrodynamics simulations indicate that they can drive powerful outflowing winds (Ohsuga et al. 2005; Ohsuga \& Mineshige 2011). As these outflows expand and adiabatically cool, their photosphere emits like a blackbody peaked in the optical. The presence of a strong super-Eddington outflow can increase the optical luminosity of a TDE by orders of magnitude, strongly enhancing detectability by optical time domain surveys (Strubbe \& Quataert 2009; Lodato \& Rossi 2011). Such an outflow would be characterized by strong UV absorption lines (Strubbe \& Quataert 2011). A qualitatively different model for the super-Eddington stage of a TDE flare would be the formation of a roughly spherical envelope, near hydrostatic equilibrium and supported by radiation pressure (Loeb \& Ulmer 1997). A more spherical geometry could be achieved if circularization is delayed by Lense-Thirring precession, and the orbital planes of the debris streams are strongly isotropized. However, achieving hydrostatic equilibrium requires a fine-tuning of $\dot{M} \approx \dot{M}_{\text {Edd }}$, and significantly super-Eddington accretion rates will lead to an expansion of the envelope that may develop into a wind rather than reaching a new equilibrium (Ulmer et al. 1998).

A final source of electromagnetic emission in TDEs is the launching of collimated relativistic jets. Such jets are seen in a wide range of accreting black hole systems, from puny galactic microquasars to the largest extragalactic blazars. Only, recently, however, has interest in TDE jets arisen, with a handful of theoretical papers (Farrar \& Gruzinov 2009; Giannios \& Metzger 2011) preceding the observational discovery of one (Bloom et al. 2011; Zauderer et al. 2011; Levan et al. 2011) or possibly two (Cenko et al. 2012b) 
relativistic TDE flares by the Swift satellite. These unexpected observational discoveries have sparked much theoretical interest in TDE jets. These jets have been studied as sources of ultra high energy cosmic rays (Farrar \& Gruzinov 2009), tools for measuring SMBH spin (Stone \& Loeb 2012a), and probes of the gaseous circumnuclear medium (De Colle et al. 2012; Metzger et al. 2012). Furthermore, the relatively clean initial conditions of a TDE makes such jets ideal laboratories to examine open questions about the roles played by net magnetic flux (Giannios \& Metzger 2011) and disk-jet misalignment (Stone \& Loeb 2012a, Chapter 6) in jet launching mechanisms.

\subsection{Observed Flares}

The first strong candidate tidal disruption flares were found by X-ray instruments in the mid-1990s (Brandt et al. 1995; Grupe et al. 1995; Bade et al. 1996). In the nearly twenty years since then, roughly twenty more events have been found. The rate of TDE detection is increasing rapidly, as is the quality of observations. Upcoming time-domain optical surveys, in particular the LSST, could discover hundreds to thousands of TDEs every year (Gezari et al. 2009; Strubbe \& Quataert 2009), finally realizing the promise of TDEs as probes of SMBH demography. In this section, we review the history of TDE observations.

\subsubsection{X-ray and UV Observations}

The satellite Röntgensatellit (ROSAT) conducted a low-cadence X-ray and EUV survey which detected several candidate tidal disruption flares (Brandt et al. 1995; Grupe et al. 


\section{CHAPTER 1. INTRODUCTION}

1995; Bade et al. 1996; Komossa \& Bade 1999; Komossa \& Greiner 1999). The long time lag between observations ( $\gtrsim 6$ months) makes it challenging to compare these early flares to detailed TDE models, but in general the strongest candidates (e.g. RX J1242.6-1119A) possessed several convincing characteristics:

- Large amplitude nuclear X-ray variability. Standard AGN vary their X-ray luminosity by factors of a few, rather than the multiple orders of magnitude expected for TDEs.

- A relatively soft X-ray spectrum, consistent with models of TDE accretion disks.

- No signs of prior host galaxy AGN activity in optical spectroscopy (e.g. no narrow line region).

From these earliest TDE candidates it was already clear that multiwavelength observations are key for distinguishing TDEs from other sources of high-energy emission. Followup HST spectroscopy helped to clarify the ROSAT candidate hosts, classifying one as a likely AGN and two others as inactive galaxies (Gezari et al. 2003). Likewise, Chandra followup observations found evidence for huge (factors of $10^{2}-10^{4}$ ) declines in the X-ray luminosity of all three strong ROSAT TDE candidates, supporting the TDE hypothesis (Halpern et al. 2004). Attempts to extrapolate a TDE rate from the ROSAT sample (Donley et al. 2002), although limited by selection effects and small number statistics, found a low rate, $\sim 10^{-5}$ galaxy $^{-1} \mathrm{yr}^{-1}$, broadly consistent with theoretical expectations (Magorrian \& Tremaine 1999).

A similar X-ray survey performed with XMM-Newton identified two more likely TDE flares (Esquej et al. 2008) with the aid of X-ray and optical followup. Other XMM 
CHAPTER 1. INTRODUCTION

TDE candidates were later detected (Saxton et al. 2012), while $X M M$ followup was also used to strengthen the case for ROSAT flares (Komossa et al. 2004). In the UV, the GALEX satellite identified three candidate TDE flares (Gezari et al. 2006, 2008, 2009), all of which seemed to originate from inactive galactic nuclei. All of the GALEX flares were fit surprisingly well by $t^{-5 / 3}$ decay laws and simple blackbody spectra, and the masses inferred from simple TDE models, while somewhat imprecise, were in agreement with galaxy scaling relations. The large fitted size of the emitting blackbodies, $\sim 10 R_{\mathrm{t}}$, may imply a large shell of matter reprocessing emission from the inner disk, as in Loeb \& Ulmer (1997); Ulmer et al. (1998).

\subsubsection{Optical Detection of TDEs}

At longer wavelengths, two TDE candidates have been found (van Velzen et al. 2011b) in Sloan Digital Sky Survey (SDSS) archival searches (in combination with GALEX archival data). SDSS data combined with followup spectroscopy has also found a sample of seven extreme coronal line emitters (ECLEs), which can be interpreted as light echoes of recent SMBH flares, possibly originating in stellar tidal disruption (Komossa et al. 2009; Wang et al. 2011, 2012). Although this novel spectroscopic diagnostic shows great promise as a way to detect TDEs, and to probe the central gas distributions of distant galaxies, it remains relatively unexplored from a theoretical perspective. Due to selection effects, it is also difficult to correlate against other samples of TDE candidates; most prior searches explicitly discarded TDE candidates with strong line emission as a cut against pre-existing AGN (Wang et al. 2012). An improved theoretical understanding of the light echoes generated by TDEs would help validate the ECLE sample of TDE 
candidates.

The advent of time domain optical astronomy raises the prospect of a large, high cadence TDE sample. However, optically-selected TDE flares are more susceptible to confusion with (intrinsically more common) nuclear Type II supernovae, and also depend sensitively on the uncertain details of super-Eddington accretion. One such TDE candidate found by the Palomar Transient Factory was observed to decay very quickly, over $\approx 10$ days (Cenko et al. 2012a). If this was in fact a tidal disruption flare, it emphasizes the need for high cadence optical surveys. Very recently, the discovery of a UV/optical flare with GALEX and Pan-STARRS data has provided our most detailed look at a presumptive TDE (Gezari et al. 2012). The excellent time resolution of this event's observations allows detailed comparisons to TDE models. The authors even compare the "rising" portion of the light curve to predictions about the effects of stellar structure variation across the main sequence (Lodato et al. 2009). The most mysterious aspect of this TDE candidate, however, is the presence of helium lines and the absence of hydrogen lines. Tidally disrupting helium stars at a significant fraction of the total TDE rate is a nontrivial dynamical challenge, but so too is hiding the hydrogen from the disruption of a main sequence star.

\subsubsection{Relativistic TDEs}

Finally, there appears to be a subset of TDE flares capable of emitting powerful relativistic jets; if seen on-axis, these blazar-like objects can be observed at cosmological distance in hard X-rays and soft $\gamma$-rays. The 2012 discovery of two such relativistic TDEs by the Swift satellite has generated much interest in these events, as they offer a new 


\section{CHAPTER 1. INTRODUCTION}

TDE detection method, probe the central regions of distant galactic nuclei, and serve as laboratories for studying jet launching mechanisms. The first event, Swift J1644+57, was observed in the nucleus of a galaxy at a redshift of $\mathrm{z}=0.354$, and has been widely interpreted as the onset of a tidal disruption flare (Bloom et al. 2011; Burrows et al. 2011; Levan et al. 2011) due to rapid variability and lack of prior activity, although alternative explanations exist (Quataert \& Kasen 2012). Despite optical nondetection of this flare (Bloom et al. 2011), possibly due to dust extinction, Swift J1644+57 has been observed in radio wavelengths (Zauderer et al. 2011), where the late time radio light curve indicates jet interaction with circumnuclear gas (Zauderer et al. 2013). The second event, Swift J2058+05, was seen at a redshift of $z=1.1853$ (Cenko et al. 2012b). Unlike Swift J1644+57, this event was bright in the optical and so far lacks a detected host galaxy.

Even off-axis, the late-time radio afterglows of these jets may be detectable (although Doppler boosting will reduce the observed flux). Very Large Array (VLA) followup observations of seven previously detected TDE candidates all failed to detect 5 GHz radio emission (van Velzen et al. 2013). Different VLA observations of seven X-ray selected TDE candidates found two possible radio counterparts (Bower et al. 2013), possibly indicating a dichotomy in TDE jet production, with unknown variables (such as SMBH spin, or spin-orbit alignment) dictating whether or not jets occur.

Taken together, observations at all wavelengths provide convincing evidence for tidal disruption flares in rough agreement with our first-order theoretical picture. The onus is now on theorists to improve existing models for the rates and properties of these flares, both to better understand our existing sample of events and to maximize the scientific yield of future surveys. Only by combining future observations with improved theoretical 
CHAPTER 1. INTRODUCTION

modeling can we use these dramatic events to probe GR phenomena, and study SMBH demographics across cosmic time. 


\section{Chapter 2}

\section{Prompt Tidal Disruption of Stars as}

\section{an Electromagnetic Signature of}

\section{Supermassive Black Hole}

\section{Coalescence}

N. Stone \& A. Loeb The Monthly Notices of the Royal Astronomical Society, Vol. 412, pp. $75-80,20011$

\section{Abstract}

A precise electromagnetic measurement of the sky coordinates and redshift of a coalescing

black hole binary holds the key for using its gravitational wave (GW) signal to constrain 
cosmological parameters and to test general relativity. Here we show that the merger of $\sim 10^{6-7} M_{\odot}$ black holes is generically followed by electromagnetic flares from tidally disrupted stars. The sudden recoil imparted to the merged black hole by GW emission promptly fills its loss cone and results in a tidal disruption rate of stars as high as $\sim 0.1 \mathrm{yr}^{-1}$. The prompt disruption of a star within a single galaxy provides a unique electromagnetic flag of a recent black hole coalescence event, and sequential disruptions could be used on their own to calibrate the expected rate of GW sources for pulsar timing arrays or the proposed Laser Interferometer Space Antenna.

\section{$2.1 \quad$ Introduction}

Recently, general relativistic simulations demonstrated that the coalescence of a black hole binary is accompanied by the anisotropic emission of gravitational radiation, causing a typical recoil of hundreds of $\mathrm{km} \mathrm{s}^{-1}$ for the black hole remnant (Pretorius 2005; Baker et al. 2006; Campanelli et al. 2006). Binaries of supermassive black holes (SMBH) are a generic consequence of galaxy mergers (Escala et al. 2005; Mayer et al. 2007; Callegari et al. 2009; Colpi \& Dotti 2009), and the resultant gravitational waves (GWs) are potentially detectable with the proposed Laser Interferometer Space Antenna $(\text { LISA })^{1}$ or existing Pulsar Timing Arrays (PTAs) such as NANOGrav².

LISA would be most sensitive to binary mergers with a total mass $M_{\mathrm{BH}} \sim 10^{6-7} M_{\odot}$ (McWilliams et al. 2010), whereas the PTA sensitivity peaks at $\sim 10^{8} M_{\odot}$ (Sesana et al.

\footnotetext{
${ }^{1}$ http://lisa.nasa.gov/

${ }^{2}$ http://nanograv.org/
} 


\section{CHAPTER 2. TDES AS PROMPT EM SIGNATURE OF SMBH MERGER}

2009). PTAs have a significantly poorer localization ability, with a typical uncertainty (Sesana \& Vecchio 2010) of $\sim 40$ compared to $\lesssim 1$ square degrees for LISA. These positional errors limit the precise determination of the luminosity distance to merging binaries. An electromagnetic (EM) counterpart would greatly reduce the positional error to sub-arcsecond scales, and also determine the redshift of the source, which would enable its use as a "standard siren" (independent of the cosmic distance ladder) for precision measurements of the dark energy equation of state (Holz \& Hughes 2005; Arun et al. 2009; Schutz 2009; Bode et al. 2010).

For these reasons, prompt EM signals are of primary importance for realizing the full potential of GW cosmology. The proposed prompt EM signals have so far all assumed the uncertain presence of a circumbinary accretion disk prior to coalescence. Dissipation of GW energy in the disk might result in a weak EM transient shortly after the merger (Kocsis \& Loeb 2008), re-equilibration of the inner edge of the disk could create an X-ray brightening on a timescale of $10-10^{3}$ yr (Milosavljević \& Phinney 2005), and shocks produced by the GW-induced recoil might generate EM reverberations after the

recoil (Lippai et al. 2008) which may take $\sim 10^{4}$ years to dissipate as enhanced infrared luminosity (Schnittman \& Krolik 2008). It is not obvious whether these EM signals could be distinguished from the much more abundant sources of temporal variability in single SMBH quasars. Moreover, the luminosity of any circumbinary disk is expected to be significantly reduced by the cavity associated with the decoupling of the binary from the inner edge of the disk in the final stage of inspiral (Milosavljević \& Phinney 2005; Schnittman \& Krolik 2008). The disk is not expected to refill the cavity and return to its full luminosity for a time $\sim 7(1+z)\left(M_{\mathrm{BH}} / 10^{6} M_{\odot}\right)^{1.32} \mathrm{yr}$ after coalescence (Milosavljević \& Phinney 2005). On longer timescales, the portion of the accretion disk 


\section{CHAPTER 2. TDES AS PROMPT EM SIGNATURE OF SMBH MERGER}

that remains bound to the recoiled SMBH is expected to be detectable as a kinematically and eventually a spatially offset quasar (Loeb 2007; Bonning et al. 2007; Shields \& Bonning 2008; Komossa et al. 2008; Comerford et al. 2009; Shields et al. 2009), although its lifetime is limited by the supply of gas that can remain gravitationally bound to it (Blecha \& Loeb 2008).

Here we show that the tidal disruption of stars provides a prompt EM flag that does not depend on the prior existence of a gaseous disk in the vicinity of the merging binary, and can result from mergers of gas-poor galaxies. Recent observations have demonstrated that tidal disruption events (TDEs) have a generic lightcurve and emission spectrum (Donley et al. 2002; Gezari et al. 2006; Esquej et al. 2007; Gezari et al. 2008, 2009) that are distinguishable from normal quasar variability. Moreover, we find that SMBH recoil results in a sequence of TDEs over a timescale of decades and potentially years, with a rate that is $\sim 4$ orders of magnitude higher than the typical TDE rate in normal galaxies (Donley et al. 2002). The existence of TDEs accompanying SMBH mergers has been studied in the past only for long time delays $\left(\sim 10^{6}-10^{9} \mathrm{yr}\right)$ before (Ivanov et al. 2005; Chen et al. 2009) or after (Komossa \& Merritt 2008) the binary coalescence event. While previous studies have focused on mechanisms to slowly feed stars into an empty loss cone, here we show that $G W$ recoil will instantaneously shift the loss cone to a non-empty region of phase space. 


\subsection{Physics of the Loss Cone}

A star will be tidally disrupted by a $\mathrm{SMBH}$ of mass $M_{\mathrm{BH}}$ if it passes within the tidal distance,

$$
r_{\mathrm{t}}=r_{\star}\left(\eta^{2} M_{\mathrm{BH}} / m_{\star}\right)^{1 / 3},
$$

where $m_{\star}$ and $r_{\star}$ are the mass and radius of the star and $\eta$ is a dimensionless constant of order unity (Diener et al. 1995). In our discussion we adopt $\eta=1$ and assume an approximate main sequence scaling law of $r_{*} \propto m_{*}^{0.8}$, which implies $r_{\mathrm{t}} \propto m_{*}^{0.467}$ (Magorrian \& Tremaine 1999). Tidal disruption does not occur if $r_{\mathrm{t}}$ is smaller than the event horizon radius $r_{h}$, in which case the star is swallowed intact by the black hole. For non-spinning black holes and solar mass stars, TDEs are therefore possible for $M_{\mathrm{BH}} \lesssim 10^{8} M_{\odot}$. A significant black hole spin can allow for (angle-dependent) TDEs by SMBHs with $M_{\mathrm{BH}} \lesssim 7 \times 10^{8} M_{\odot}$ (Beloborodov et al. 1992). During a TDE, half of the star's mass becomes unbound, while the rest flows on Keplerian trajectories, until the associated gas streams return to pericenter and collisionally shock each other (Rees 1988; Evans \& Kochanek 1989). These gas streams return at a characteristic mass infall rate $\dot{M} \propto t^{-5 / 3}$ (roughly speaking; see Lodato et al. (2009) for a more thorough treatment) and form an accretion disk whose blackbody emission peaks in the UV or soft X-ray with luminosities comparable to a supernova (Loeb \& Ulmer 1997; Strubbe \& Quataert 2009). Other sources of emission include line radiation from the unbound debris (Kasen \& Ramirez-Ruiz 2010), and a possible brief period of super-Eddington mass fallback. These features are useful for differentiating TDE flares from supernovae or quasar variability, and some have already been applied to candidate events(Komossa 2004).

In a spherical galaxy with a stationary $\mathrm{SMBH}$, a star is tidally disrupted if its 
orbital angular momentum per unit mass falls below a critical value,

$$
J^{2}=|\vec{x} \times \vec{v}|^{2}<J_{\text {crit }}^{2} \approx 2 G M_{\mathrm{BH}} r_{\mathrm{t}},
$$

where we have approximated the relevant orbit as nearly radial. Such orbits define the so-called "loss cone". The rate of TDEs for a stationary SMBH is set by relaxation processes. Inward of a certain galacto-centric radius the loss cone is empty, but outside of it there is a "pinhole" regime where the rate of scatter in and out of the loss cone is greater than the orbital frequency (Magorrian \& Tremaine 1999).

Over the long orbital timescale of stars, the impulsive GW-induced recoil of the SMBH remnant from a binary coalescence event yields a nearly instantaneous change in the black hole velocity relative to the stars (O'Leary \& Loeb 2009). Viewed from the rest frame of the black hole, there is a sudden shift in the velocity of all stars, yielding a new loss cone defined by

$$
J^{2}=\left|\vec{x} \times\left(\vec{v}-\vec{v}_{\mathrm{k}}\right)\right|^{2}<J_{\text {crit }}^{2} \approx 2 G M_{\mathrm{BH}} r_{\mathrm{t}},
$$

where $\vec{v}_{\mathrm{k}}$ is the SMBH kick velocity.

We parametrize the density of stars around a SMBH binary in the last stages of its inspiral, using a power-law profile,

$$
\rho=\rho_{0}\left(r / r_{0}\right)^{-\gamma} .
$$

This density profile corresponds to an isotropic pre-kick distribution function of stars,

$$
f(r, v)=C\left(2 G M_{\mathrm{BH}} / r-v^{2}\right)^{\gamma-3 / 2},
$$

where the normalizing constant is given by

$$
C=\frac{(3-\gamma)(\gamma-0.5) \Gamma(\gamma+1)}{2 \pi^{2.5} \Gamma(\gamma+0.5)} \frac{M_{\mathrm{BH}}}{r_{\mathrm{inf}}^{3}}\left(\frac{r_{\mathrm{inf}}}{2 G M_{\mathrm{BH}}}\right)^{\gamma},
$$

which in turn depends on the observationally calibrated (Merritt et al. 2009) radius of 
influence of the SMBH (inside of which the mass in stars is $2 M_{\mathrm{BH}}$ ). For a full sample of galaxies this is

$$
r_{\mathrm{inf}}=24\left(M_{\mathrm{BH}} / 10^{8} M_{\odot}\right)^{0.51} \mathrm{pc},
$$

while for core galaxies alone it is

$$
r_{\mathrm{inf}}=35\left(M_{\mathrm{BH}} / 10^{8} M_{\odot}\right)^{0.56} \mathrm{pc} .
$$

Stars will be bound to the black hole system of total mass $M_{\mathrm{BH}}$ before the binary coalescence if

$$
\vec{v}^{2}<2 G M_{\mathrm{BH}} / r
$$

and after coalescence if

$$
\left(\vec{v}-\vec{v}_{k}\right)^{2}<2 G M_{\mathrm{BH}} / r .
$$

The intersection of these two spheres in velocity space, with each other and with the loss cone, is the region of phase-space containing bound stars which are tidally disrupted after the recoil. By performing a Monte Carlo integration of the appropriate distribution function over this region we calculate the number of post-recoil TDEs. Through a separate integral we find that the unbound stars provide $\lesssim 10 \%$ of the total number of TDEs and can be neglected. Another small correction to the total rate involves the net SMBH mass loss by GW emission, which is typically $\lesssim 5 \%$ of the pre-merger mass (Campanelli et al. 2006). We have included the associated small reduction (by $\sim 10 \%$ ) in the number of tidally disrupted stars. To evaluate the observability of the recoil-induced TDEs, we define the quantity $N_{<}(t)$ as the number of stars in the post-recoil loss cone which are tidally disrupted in $t$ years. Since stars which fall into the new loss cone are near their apocenter in the rest frame of the kicked black hole, $N_{<}(100)$ is the number of stars with orbital periods below 200 years. 


\section{CHAPTER 2. TDES AS PROMPT EM SIGNATURE OF SMBH MERGER}

The result of the Monte Carlo integral is sensitive to the innermost region of the distribution function, whose details are determined by the pre-merger dynamical environment. In particular, the SMBH binary will excavate a larger and more complex loss region than is given by Eq. (2.3). Below, we consider the innermost regions of phase space in gas-poor and gas-rich mergers, separately.

\subsection{Dry Mergers}

In the absence of gas, $\gamma=1.75$ is the dynamically relaxed (so-called "Bahcall-Wolf") equilibrium state (Bahcall \& Wolf 1976) of a stellar cluster around a SMBH. However, core galaxies are believed to be the end product of a binary inspiral, as the binary sheds angular momentum by ejecting stars. Numerical simulations of this process show that a binary hardening its orbit through scattering of stars will scour a core (Merritt 2006), but at some point depletion of the remaining stars in the binary loss cone (including in this context stars whose pericenters fall within twice the binary semimajor axis) will lead to a stalling of the binary and the so-called "final parsec problem" (Milosavljević \& Merritt 2001). Without gas, the binary can only merge via a re-population of its loss cone. Significant triaxiality of the galaxy potential (Merritt \& Poon 2004) tends to re-populate the binary loss-cone but preserve a core with $\gamma \approx 1$. Alternatively, collisional processes generate a central cusp of stars, though this method of binary hardening is only effective for binary masses $\lesssim 10^{7} M_{\odot}$ (Merritt et al. 2009), and it may be modified in the presence of massive perturbers (Perets \& Alexander 2008), such as infalling molecular clouds in gas-rich mergers. These gas-free scenarios lead us to consider both core galaxies where $\gamma=1$, and galaxies with a joint core-cusp density profile where an inner $\gamma=1.75$ profile 


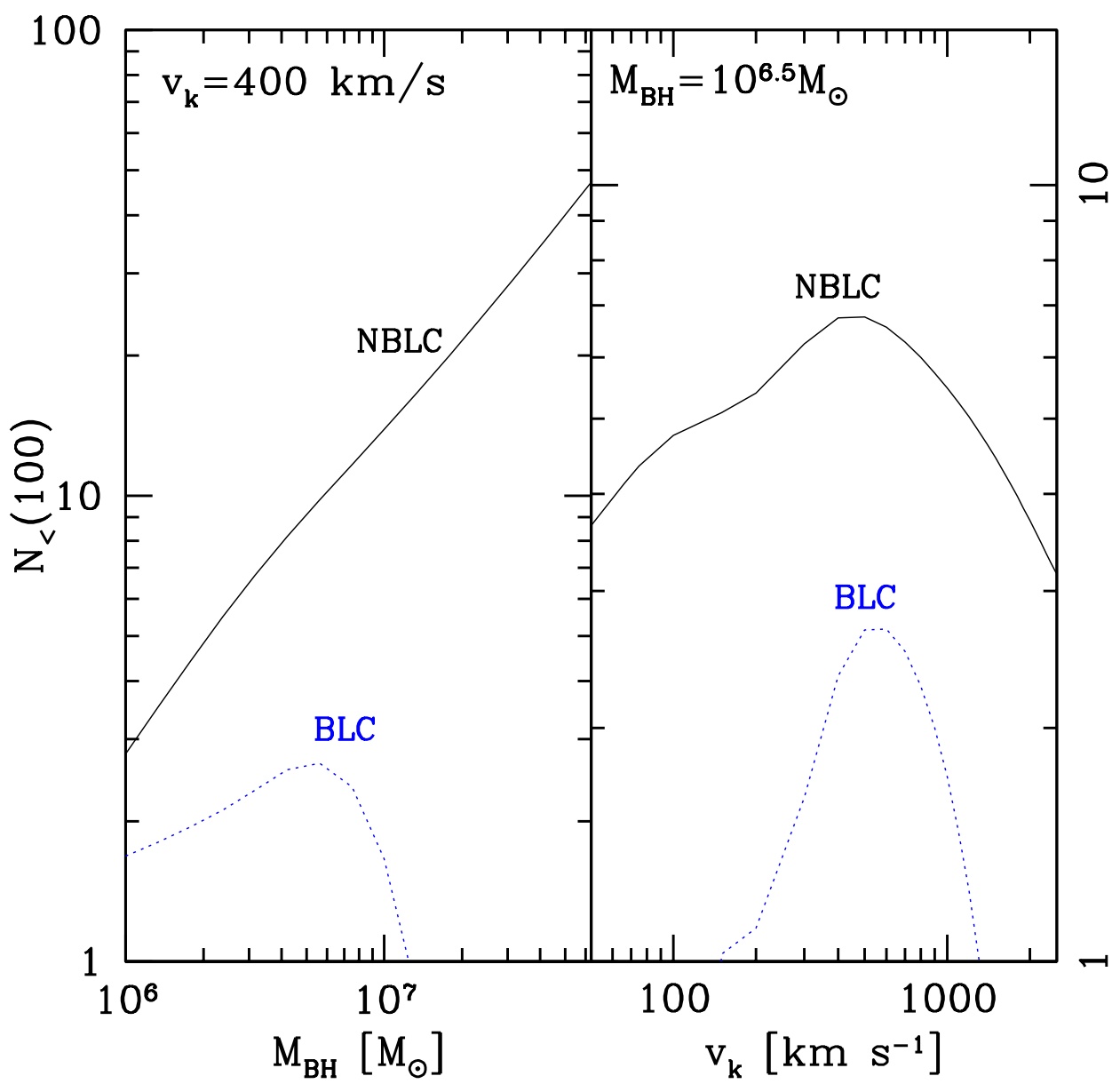

Figure 2.1.- : Expected number of stars disrupted in less than 100 years, $N_{<}(100)$, in the dry merger, joint core-cusp case. Left panel: Black hole mass dependence for kick velocity $v_{\mathrm{k}}=400 \mathrm{~km} \mathrm{~s}^{-1}$. The dotted blue line (labeled 'BLC') represents the physically realistic scenario including the binary loss cone discussed in the text, whereas the black line (labeled 'NBLC') accounts only for the loss cone of a single black hole. Right panel: Kick velocity dependence for a black hole mass $M_{\mathrm{BH}}=10^{6.5} M_{\odot}$, using the same line types. 
meets an outer $\gamma=1$ profile at a radius of $0.2 r_{\text {inf }}$ (Merritt et al. 2009). We use Equation (2.8) as the radius of influence in these models.

It is necessary to exclude the stars located in the pre-coalescence loss cone. The size of this region of phase space is somewhat uncertain. For collisional re-population of the loss cone, numerical simulations (Merritt et al. 2007) indicate the SMBH binary will decouple from a relaxed distribution of stars at a semimajor axis of $a_{\mathrm{eq}} \sim 10^{-3} r_{\text {inf }}$, and coalesce before the stars can relax into the gap left behind. To model this cavity in energy space we remove all stars with semimajor axes less than this radius. However, because relaxation in angular momentum is faster than in energy, the resultant gap in angular momentum space will be partially refilled prior to the merger. The timescale for filling up a gap in angular momentum space is given by (Merritt \& Wang 2005),

$$
T_{\text {gap }}=\frac{a}{r_{\text {inf }}} T_{\mathrm{r}},
$$

where $a$ is the semimajor axis of the SMBH binary, taken to be the pericenter at which stars are ejected (Merritt \& Wang 2005), and the system relaxation time at the radius of influence is (Merritt et al. 2009),

$$
T_{\mathrm{r}} \approx 8.0 \times 10^{9} \mathrm{yr}\left(\frac{M_{\mathrm{BH}}}{10^{6} M_{\odot}}\right)^{1.54} .
$$

Thus, a second cavity in the distribution function is created by removing all stars with pre-kick pericenters lower than the binary separation $a$ at which $T_{\text {gap }}$ equals the gravitational wave timescale,

$$
T_{\mathrm{GW}}=\frac{5 c^{5} a^{4}}{256 G^{3} M_{\mathrm{BH}}^{2} \mu},
$$

with $\mu=M_{1} M_{2} / M_{\mathrm{BH}}$ being the reduced mass of the binary and $M_{\mathrm{BH}}=\left(M_{1}+M_{2}\right)$.

For simplicity, we adopt a flat $J$ dependence for $f(E, J)$ with the cuts mentioned above. In the classical loss cone calculation, the steady state solution of the orbit-averaged 
Fokker-Planck equation yields a distribution function that varies logarithmically with $J$ at fixed $E$ (Cohn \& Kulsrud 1978). However, this solution may not apply to the innermost stars, for which the orbit averaged assumption may break down and strong star-star scatterings (which the Fokker-Planck approach does not account for) could be

important. Furthermore, the loss cone of a binary SMBH is not the pure sink assumed for a single $\mathrm{BH}$, since stars may remain bound to the binary on low- $J$ orbits. The use of a logarithmic instead of a flat distribution would have reduced $N_{<}(t)$ by a factor of $\sim 2-4$.

We use these cuts in stellar energy and angular momentum as modifications to the joint core-cusp profile. We generalize the results to a range of stellar masses using a Salpeter initial mass function (IMF) with a differential number of stars, $d N_{\star} / d m_{\star} \propto m_{\star}^{-2.35}$ in the mass range $0.1 M_{\odot}<m_{\star}<100 M_{\odot}$. The number of disruptions is dominated by low mass stars despite their smaller $r_{\mathrm{t}}$; switching to a top-heavy IMF, as is sometimes discussed in the context of galactic nuclei (Bartko et al. 2010), would reduce $N_{<}(100)$ by a factor of a few.

\subsection{Wet Mergers}

In gas-rich mergers, the pre-kick profile is likely to be different. On the one hand, rapid loss of angular momentum by the binary to dynamical friction on the gas can produce a core by denying stars the time needed to relax into a central cusp as described above (Merritt et al. 2009); but on the other hand, in situ star formation could rebuild a nuclear cusp while the binary orbit hardens. The possibility of star formation, and subsequent migration, in disks motivates us to consider values of $\gamma=1.5,1.75,2$. Alternatively, stars 


\section{CHAPTER 2. TDES AS PROMPT EM SIGNATURE OF SMBH MERGER}

formed elsewhere could be "ground down" into orbits inside the disk (Syer et al. 1991), and then behave in a similar fashion. Although the details of star formation in disks fragmenting due to gravitational instability are quite complex (Shlosman \& Begelman 1987; Alexander et al. 2008), we provide an approximate description of their potential to contribute to the post-kick loss cone here. If the Roche radius of the star in the disk exceeds the disk scale height, and tidal coupling of the star to the disk is at least as effective as viscosity at transporting angular momentum, the star will open a gap in the disk (Syer et al. 1991) and migrate inward on a viscous timescale, to the point where those conditions are no longer met, or the disk's inner edge (Goodman \& Tan 2004), whichever is larger. Here we use Equation (2.7) for the radius of influence, since we are considering galactic nuclei in the process of rebuilding their cusps.

We follow a similar procedure as with dry mergers, to approximate the size of the pre-merger loss cone. Assuming a thin disk, the radial size of the central cavity is determined by setting the viscous timescale at the radius of marginal self-gravity (Goodman \& Tan 2004),

$$
T_{\text {vis }}=4.2 \times 10^{5} \mathrm{yr} \alpha_{0.3}^{-1 / 3} \kappa^{-1 / 2} \mu^{1 / 3}\left(\frac{\epsilon_{0.1}}{l_{E}}\right)^{1 / 6} M_{8}^{1 / 2},
$$

equal to $T_{\mathrm{GW}}$. Here, $\kappa$ is the opacity in units of electron-scattering opacity, $\mu$ is mean gas particle mass in units of the proton mass, $\alpha_{0.3}$ is the standard (Shakura-Sunyaev) viscosity parameter scaled to $0.3, \epsilon_{0.1}$ is the radiative efficiency scaled to $10 \%$, and $l_{E}$ is the total radiated luminosity in units of the Eddington limit for the black hole mass $M_{\mathrm{BH}}=M_{8} \times 10^{8} M_{\odot}$. Noting the weak power law dependences in $T_{\mathrm{vis}}$, we set all parameters except $M_{\mathrm{BH}}$ to their fiducial values (Goodman \& Tan 2004). We remove any stars with pericenters interior to the radius at which the SMBH binary decouples 


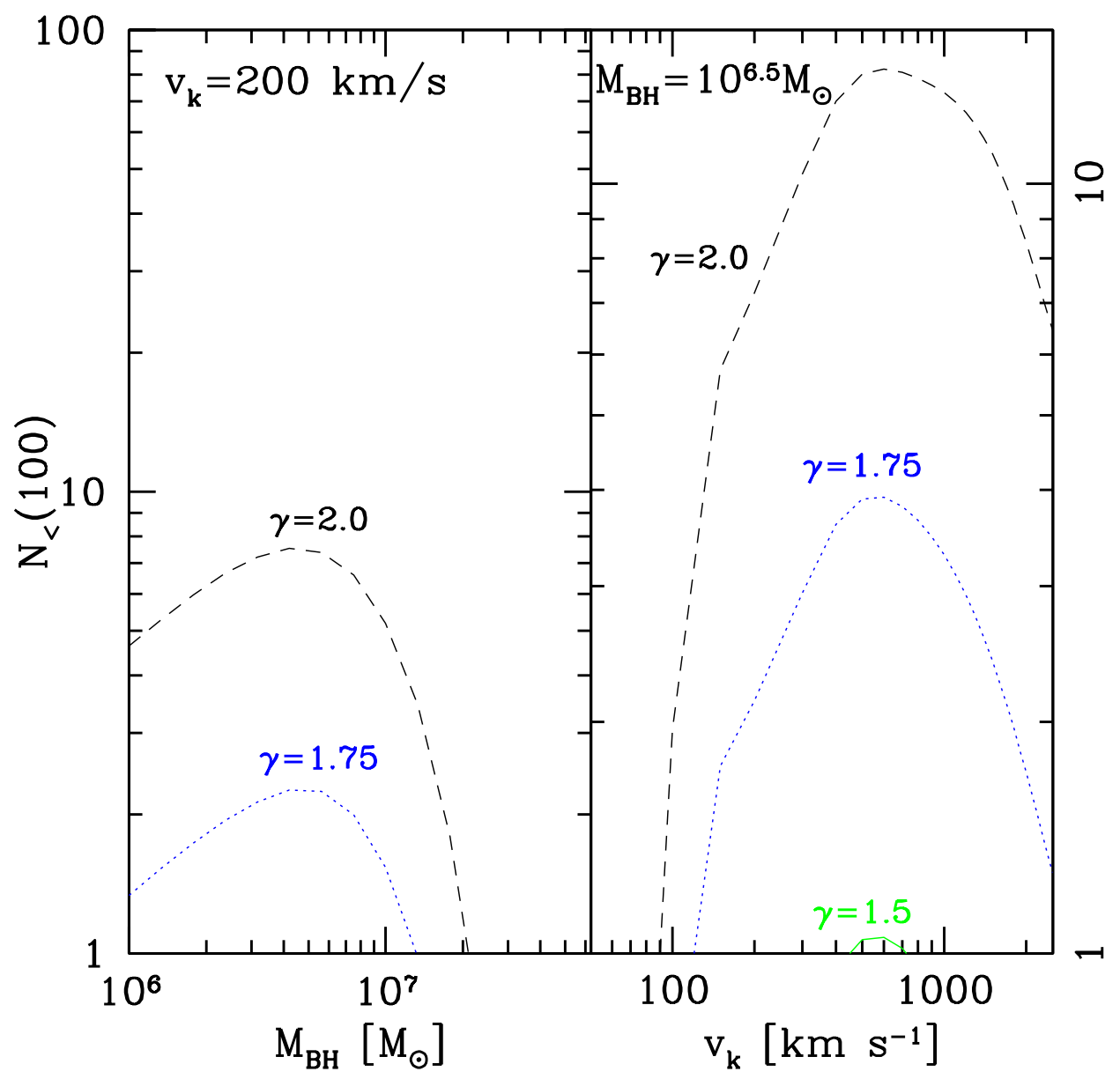

Figure 2.2.-: Expected number of stars disrupted in less than 100 years, $N_{<}(100)$, in the wet merger scenario. Left panel: Mass dependence with $v_{\mathrm{k}}=200 \mathrm{~km} \mathrm{~s}^{-1}$. The solid green line represents a $\gamma=1.5$ cusp, the dotted blue $\gamma=1.75$, and the dashed black $\gamma=2.0$. Right panel: Velocity dependence with $M_{\mathrm{BH}}=10^{6.5} M_{\odot}$, and the same lines as in the left panel. 
from the accretion flow, and assume that the remaining stars obtain a nearly spherical angular distribution (similarly to the innermost S-stars in the Milky-Way nucleus (Ghez et al. 2008)) before the SMBH recoil. Because the details of star formation within the disk are highly uncertain and the TDE rate is dominated by low-mass stars, we assume for simplicity $m_{\star} \sim 1 M_{\odot}$ in the wet merger case. We emphasize that in this case, accretion-induced alignment of SMBH spins prior to merger is expected to strongly suppress kicks over $\sim 200 \mathrm{~km} \mathrm{~s}^{-1}$ (Bogdanović et al. 2007; Dotti et al. 2010).

\subsection{Other Considerations}

Other processes could also partially refill the binary loss cone. In analogy to the problem of resonant capture during planetary migration (Yu \& Tremaine 2001), mean-motion resonances could be capable of pulling stars inward during the final stages of the SMBH merger, dramatically increasing the number of post-kick disruptions. The special case of the 1:1 Lagrange point resonance has recently been investigated (Schnittman 2010) and found capable of migrating stars to within tens of Schwarzschild radii from the system barycenter (Seto \& Muto 2010). Higher integer ratio mean-motion resonances have been seen to affect stars (see Chen et al. (2009), Figs. 5, 6, 7) as a binary SMBH hardens, although their ability to drive resonant migration is less clear. A detailed study of resonant migration in SMBH binaries is beyond the scope of this paper, but this effect has the potential to dramatically expand the short-period population of the post-kick loss cone.

For a source at a redshift $z$, cosmological time dilation will stretch the duration of each TDE flare, delay the onset of the first post-kick flare, and reduce the observed TDE 


\section{CHAPTER 2. TDES AS PROMPT EM SIGNATURE OF SMBH MERGER}

rate all by the same factor of $(1+z)$. However, observations suggest that mean density of stars in high-redshift galaxies scales as $(1+z)^{3}$ (Oesch et al. 2010). This could lead to a net enhancement in the observed TDE rate $\propto(1+z)^{2}$ per galaxy, if the central regions of galaxies are self-similar. We set $z=0$ to ignore these possible cosmological effects in our calculated TDE rates.

\subsection{Results}

For simplicity, our calculations assume binaries of equal mass black holes. An unequal mass would increase $T_{\mathrm{GW}}$ and increase $N_{<}(t)$ by allowing more time for refilling the binary loss cone, but it would also decrease the likely values of $v_{k}$. The latter change dominates only for mass ratios smaller than $\sim 0.1$, so our results should be regarded as conservative for major mergers. Figure 2.1 presents the velocity and mass dependences of our most realistic model (the joint core-cusp profile, for SMBH binaries that harden in dry mergers by scattering of stars), as well as a less realistic core-cusp model included for illustrative purposes. The first model (labeled BLC) removes stars from the binary loss cone and results in an interesting number of TDEs for $M_{\mathrm{BH}} \lesssim 10^{7} M_{\odot}$ and $200 \mathrm{~km} \mathrm{~s}^{-1} \lesssim v_{\mathrm{k}} \lesssim 1000 \mathrm{~km} \mathrm{~s}^{-1}$. At low velocities, overlap with the pre-merger loss cone sharply suppresses $N_{<}(100)$, while at high velocities, the reduced size of the bound stellar population also shrinks $N_{<}(100)$. At higher masses, all short-period stars are scoured by the pre-merger binary loss cone. The second case in Figure 2.1 (labeled NBLC) replaces the loss cone of a binary with that of a single black hole. Interestingly, the dramatic increase in $N_{<}(100)$ here results from the addition of relatively few stars ( $\sim 100$ for $\left.M_{\mathrm{BH}}=10^{6} M_{\odot}\right)$, indicating that resonant migration of a small population 


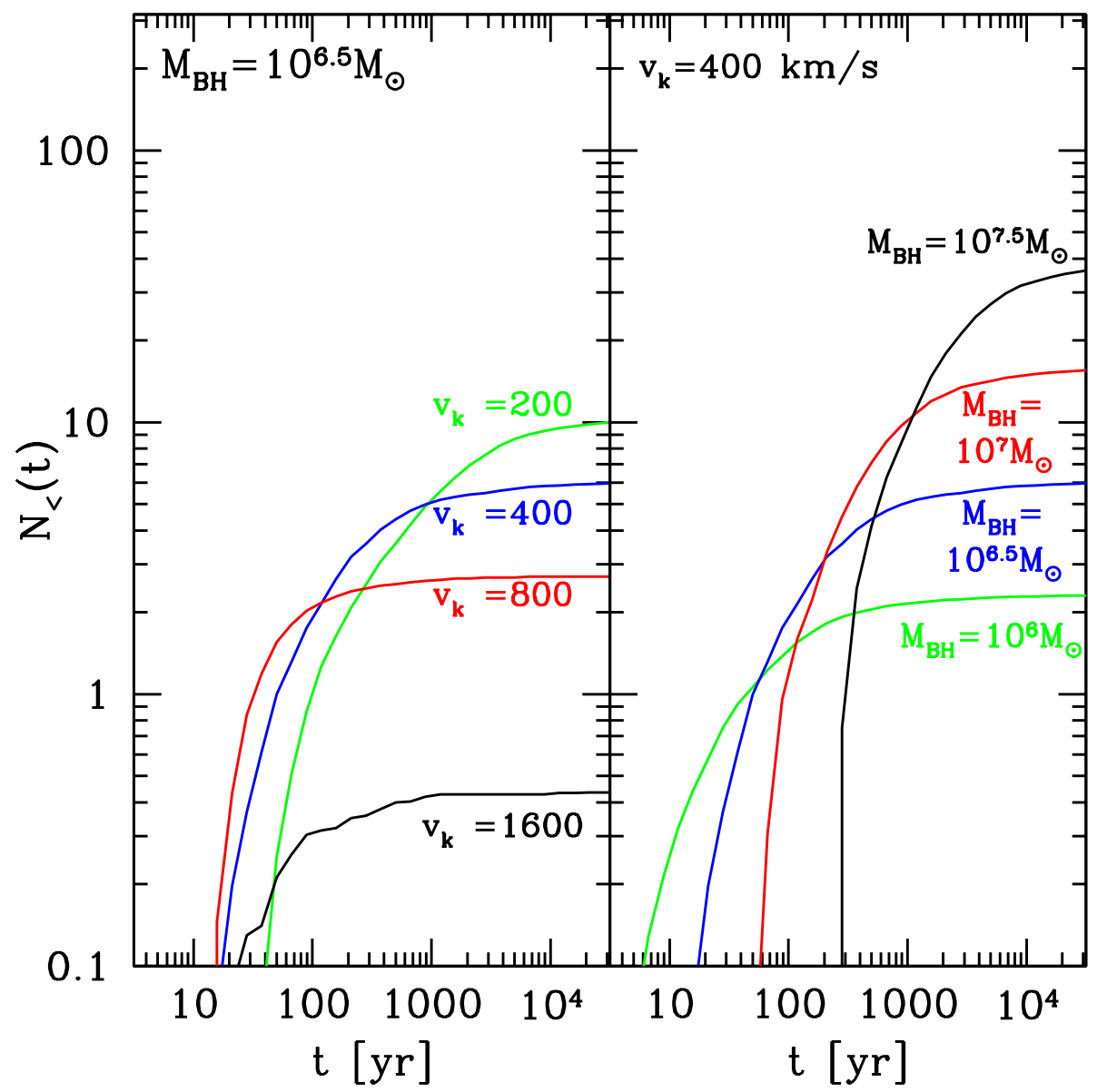

Figure 2.3.-: Left panel: Expected number of stars disrupted in less than $t$ years, $N_{<}(t)$, for the dry merger model and $M_{\mathrm{BH}}=10^{6.5} M_{\odot}$. The green line is for $v_{\mathrm{k}}=200 \mathrm{~km} \mathrm{~s}^{-1}$, the blue line is for $v_{\mathrm{k}}=400 \mathrm{~km} \mathrm{~s}^{-1}$, the red line is for $v_{\mathrm{k}}=800 \mathrm{~km} \mathrm{~s}^{-1}$, and the black line is for $v_{\mathrm{k}}=1600 \mathrm{~km} \mathrm{~s}^{-1}$. Right panel: $N_{<}(t)$ for $v_{\mathrm{k}}=400 \mathrm{~km} \mathrm{~s}^{-1}$ and varying masses. The green line is for $M_{\mathrm{BH}}=10^{6} M_{\odot}$, the blue for $M_{\mathrm{BH}}=10^{6.5} M_{\odot}$, the red for $M_{\mathrm{BH}}=10^{7} M_{\odot}$, and the black for $M_{\mathrm{BH}}=10^{7.5} M_{\odot}$. 


\section{CHAPTER 2. TDES AS PROMPT EM SIGNATURE OF SMBH MERGER}

of stars could significantly boost $N_{<100}$. Finally, the $\gamma=1$ (pure core) case does not have enough centrally located stars to produce post-kick tidal disruptions on $<100$ yr timescales.

The results for wet mergers are illustrated in Figure 2.2. Although these rely on significantly more uncertain assumptions than the dry merger model, they show that star formation can produce interesting values of $N_{<100}$ in the $\gamma=1.75$ and $\gamma=2$ cases, with rates high enough that sequential TDEs could be observed in a single galaxy.

Figure 2.3 shows how the delay until the first post-kick disruptions changes with black hole mass and kick velocity. Here we consider the joint core-cusp model (with removal of pre-merger loss cone by the binary), and find that the first disruption is expected to occur between three and five decades after SMBH coalescence for black holes with masses between $10^{6} M_{\odot}$ and $10^{7} M_{\odot}$, and kick velocities between 400 and $800 \mathrm{~km} \mathrm{~s}^{-1}$. Fortunately, this region of parameter space falls within both the black hole mass range $L I S A$ is likely to observe, and the range of physically plausible recoil velocities for dry mergers. In the most event-rich wet merger scenarios, the first TDEs could occur as soon as $\sim 1$ yr after coalescence.

\subsection{Summary}

We find that merging galaxies with black hole masses $M_{\mathrm{BH}} \lesssim 10^{7} M_{\odot}$ are likely to produce a tidal disruption flare a few decades after coalescence in the case of a gas-poor merger. Multiple flares could possibly be seen on a timescale of years if resonant migration is effective. For gas-poor mergers, the peak rate is therefore at least $\sim 10^{4}$ times higher 
than the typical TDE rate in galaxies (Donley et al. 2002). The total number of TDEs is maximized, and delay until the first TDE minimized, if the kick velocity is in the range of $200-1000 \mathrm{~km} \mathrm{~s}^{-1}$.

Our minimal predictions concerning dry mergers could be dramatically enhanced if resonant migration increases the number and frequency of post-kick tidal disruptions. The case of wet mergers is substantially more complicated, and because star formation and disk migration can also significantly increase the population of the post-kick loss cone, a much more detailed study of star-disk interactions around binary SMBHs is needed to make robust predictions in the gas-rich case.

Moderate to high kick velocities in dry mergers will provide a robust EM counterpart to the GW signature of black hole coalescence within the LISA band, enabling accurate identification of the host galaxy and a precise measurement of cosmological parameters (Holz \& Hughes 2005) within a few decades of the initial GW signal. With the advent of massive transient surveys, such as $\mathrm{PTF}^{3}$, Pan-STARRS ${ }^{4}$, and $\mathrm{LSST}^{5}$, it is possible that sequential tidal disruption flares could flag black hole recoil events without a GW signal, providing an independent test of the strong field regime of general relativity and a calibration of the expected event rate for LISA and PTAs.

\footnotetext{
${ }^{3}$ http://www.astro.caltech.edu/ptf/

${ }^{4}$ http://pan-starrs.ifa.hawaii.edu/public/

${ }^{5}$ http://www.lsst.org/lsst
} 


\section{Chapter 3}

\section{Tidal Disruption Flares of Stars}

\section{From Moderately Recoiled Black}

\section{Holes}

N. Stone \& A. Loeb The Monthly Notices of the Royal Astronomical Society, Vol. 422, pp. 1933-1947, 2012

\section{Abstract}

We analyze stellar tidal disruption events as a possible observational signature of gravitational wave induced recoil of supermassive black holes. As a black hole wanders through its galaxy, it will tidally disrupt bound and unbound stars at rates potentially observable by upcoming optical transient surveys. To quantify these rates, we explore a broad range of host galaxy and black hole kick parameters. We find that emission from 
a transient accretion disk can produce $\sim 1$ event per year which LSST would identify as spatially offset, while super-Eddington outflows, if they exist, are likely to produce $\sim 10$ spatially offset events per year. A majority of tidal disruption flares, and a large majority of flares with an observable spatial offset, are due to bound rather than unbound stars. The total number of disruption events due to recoiled black holes could be almost $1 \%$ of the total stellar tidal disruption rate.

\section{$3.1 \quad$ Introduction}

Recent advances in numerical general relativity quantified how the coalescence of unequal black hole binaries leads to the anisotropic emission of gravitational radiation, which can carry enough linear momentum to deliver a substantial kick to the merged black hole (Pretorius 2005; Baker et al. 2006; Campanelli et al. 2006). Because the inspiral and eventual coalescence of supermassive black holes (SMBHs) is a frequent consequence of galaxy mergers, it is expected that SMBH recoil will often accompany these events. The magnitude of the recoil varies as a function of the initial mass ratio and the spin and orbital angular momentum vectors of the coalescing black holes, but is generally of order hundreds of kilometers per second (Schnittman \& Buonanno 2007; Lousto et al. 2010a,b). At the high end of the velocity distribution, the black hole can recoil with a

velocity $\gtrsim 1000 \mathrm{~km} \mathrm{~s}^{-1}$ and escape the merged galaxy. More commonly, it will oscillate for a time ranging from $10^{6}-10^{9}$ yrs (Blecha \& Loeb 2008; Sijacki et al. 2011; Blecha et al. 2011) before settling down into the galaxy's center.

Observation of a recoiling SMBH would be of interest both as a probe of hierarchical galaxy evolution and as a test of the predictions of strong field general relativity. 


\section{CHAPTER 3. OFFSET TDES FROM RECOILED SMBHS}

Consequently, several papers have focused on observational signatures of a recoiling SMBH. A black hole kicked at a substantial fraction of its host galaxy's escape velocity can create or expand a low-density stellar core (Gualandris \& Merritt 2008). The small cloud of stars gravitationally bound to the recoiling black hole would appear like a globular cluster, but with much higher velocity dispersion (O'Leary \& Loeb 2009; Merritt et al. 2009; O'Leary \& Loeb 2012). Gas accretion onto the black hole, manifested as a spatially or kinematically offset quasar (Madau \& Quataert 2004; Loeb 2007), is potentially a very clear signature, but the gas reservoir bound to the black hole will be depleted within $10^{7}$ years after ejection from the center of its galaxy (Blecha \& Loeb 2008; Blecha et al. 2011). Several candidates have already been detected with this last method (Komossa et al. 2008; Shields et al. 2009; Civano et al. 2010), although the evidence for black hole recoil is not conclusive (Bogdanović et al. 2009). A potentially longer-lived source of accretion power is the tidally disrupted matter from stars passing too close to the recoiling black hole, which could be visible as an off-center tidal disruption flare. Because tidal disruption flare lightcurves have, in principle, several unique identifiers (Strubbe \& Quataert 2009; Guillochon et al. 2009; Kasen \& Ramirez-Ruiz 2010; Strubbe \& Quataert 2011), an off-center or intergalactic tidal disruption flare would be a very strong indication of a recoiling black hole. This scenario was first investigated in a paper by Komossa \& Merritt (2008) (hereafter KM08). However, to evaluate the utility of tidal disruption signatures for recoiling black holes it is first necessary to obtain estimates for the tidal disruption rates produced by these systems. KM08 calculate these rates for relatively large kick velocities, while in this paper we generalize the calculation to cover realistic, and often moderate, kick velocity distributions. The aim of these calculations is to approximate the frequency of off-center and spectrally shifted tidal disruption flares, 


\section{CHAPTER 3. OFFSET TDES FROM RECOILED SMBHS}

to determine if tidal disruption events (TDEs) are useful probes of physically motivated kick velocity distributions. These rate calculations are also relevant to SMBHs ejected from galaxy centers in triple-SMBH interactions (Hoffman \& Loeb 2007), although we do not attempt to evaluate the more complicated distributions of parameters for such events.

Very large increases in tidal disruption rates have been discussed previously in the context of galaxy mergers, both as a prompt electromagnetic signal immediately following SMBH coalescence (Stone \& Loeb 2011), and due to resonances or chaotic stellar orbits at the end of the dynamical friction phase of an SMBH binary (Ivanov et al. 2005; Chen et al. 2009; Wegg \& Nate Bode 2011; Chen et al. 2011). Neither of these mechanisms is capable of producing a tidal disruption flare with an observable spatial offset, as both can only occur in galactic nuclei. Tidal flares produced by wider SMBH binaries could still be a source of confusion in interpreting spatially offset TDEs, however. Binary-produced flares would themselves be of interest, but would not be directly useful for testing predictions of gravitational wave recoil. Distinguishing between the two possibilities will be simple in some cases: for instance, a TDE in intergalactic space, independent of an observable stellar population, would likely be due to a recoiling black hole; whereas a TDE with periodic interruptions in its lightcurve (Liu et al. 2009) would be due to a hard SMBH binary. A softer SMBH binary could perhaps be distinguished from a recoiling SMBH by archival or post-flare searches for a second optical nucleus. However, in many cases it will be not be trivial to disentangle the two causes, and detailed observation and modeling of the host galaxy could be necessary to determine if it is likely to harbor a binary SMBH at the observed spatial or kinematic offset. A final source of confusion could be disruption of stars by IMBHs left over in a 


\section{CHAPTER 3. OFFSET TDES FROM RECOILED SMBHS}

galaxy's halo from earlier stages of hierarchical growth (O'Leary \& Loeb 2012). However, these TDEs would probably be distinguishable due to their low black hole masses, and in any event would be a complementary example of black hole recoil.

Although the current sample of observed TDEs is small, with roughly a dozen strong candidates (Gezari et al. 2009), current and upcoming time-domain optical transient surveys such as Pan-STARRS ${ }^{1}, \mathrm{PTF}^{2}$, and $\mathrm{LSST}^{3}$ are expected to increase that sample by $1-3$ orders of magnitude (Strubbe \& Quataert 2009), making it feasible to study sub-populations of TDEs. If sufficiently large, the subset of disruption flares associated with recoiling black holes could be used to constrain the LISA ${ }^{4}$ event rate or the distribution of kick velocities associated with astrophysical SMBH mergers; at the very least, detection of this subset of TDEs could qualitatively confirm recent numerical relativity predictions.

The outline of this paper is as follows. In $\S 2$ we develop the model used to estimate the TDE rate over a wide range of kick velocities and galaxy parameters, and in $\S 3$ we explain in more detail the distributions of disruption properties we integrate over. In $\S 4$ we discuss the results of our modeling, and in $\S 5$ we offer our conclusions on the viability of tidal disruption flares as a technique for identifying recoiled SMBHs.

\footnotetext{
${ }^{1}$ http://pan-starrs.ifa.hawaii.edu/public/

${ }^{2}$ http://www.astro.caltech.edu/ptf/

${ }^{3}$ http://www.lsst.org/lsst

${ }^{4}$ http://lisa.nasa.gov/
} 


\subsection{Model}

The three primary quantities to be calculated are the trajectory of the kicked black hole through its host galaxy, the rate at which it disrupts unbound stars that it encounters, and the rate at which it depletes its cloud of gravitationally bound stars via tidal disruption. The location (and possible kinematic offset) of a disruption event relative to its host galaxy will determine whether the flare can be distinguished from a TDE due to a stationary black hole. $§ 2.1$ examines expected ranges of black hole kicks, $\S 2.2$ discusses the relevant tidal disruption physics, $\S 2.3$ lays out the galaxy parametrization used in this paper, and $\S 2.4$ and $\S 2.5$ describe the techniques used to estimate TDE rates for unbound and bound stars, respectively. Finally, in $\S 2.6$ we discuss observational constraints. Our general strategy is as follows: Strubbe \& Quataert (2009) (hereafter SQ09) calculate the number of TDEs a generic survey would be expected to detect, binned by black hole mass (see SQ09 Figures 9, 13). In this calculation they assume a

time-averaged tidal disruption rate $\dot{N}=10^{-5} \mathrm{yr}^{-1}$ galaxy ${ }^{-1}$. Our model averages over a kick velocity distribution and a range of galaxy parameters (detailed in $§ 2.3$ ) to calculate a $\dot{N}$ dependent on black hole mass, and then modifies the SQ09 survey calculations accordingly.

\subsubsection{Black Hole Kicks}

The gravitational recoil velocity of a post-merger SMBH depends only on the mass ratio, spin amplitudes, and spin orientations (relative to the orbital angular momentum plane) of the two progenitor SMBHs. The merger of Schwarzschild black holes represents a simple case, with a maximum kick velocity of $\sim 175 \mathrm{~km} \mathrm{~s}^{-1}$ occurring at a mass ratio of 


\section{CHAPTER 3. OFFSET TDES FROM RECOILED SMBHS}

0.36 (González et al. 2007). As the dimensionless spin parameters of the two SMBHs $a_{1}, a_{2}$ increase, so does the maximum kick velocity $v_{\mathrm{k}}$, although it has complicated functional dependences on the relative inclination of the pre-merger spin axes and the orbital angular momentum axis. An exact calculation requires the full framework of numerical relativity, but analytic fitting formulae can be calibrated to numerical relativity results, recently yielding quite high accuracy (Lousto et al. 2010a). Although certain combinations of SMBH binary initial parameters can produce kicks up to $\sim 4000 \mathrm{~km} \mathrm{~s}^{-1}$ (Campanelli et al. 2006), observation constrains how frequent such high-velocity kicks can be, due to the fact that galaxies with bulges all seem to possess central SMBHs (Ferrarese \& Ford 2005; Blecha et al. 2011). This constraint is weakened, however, by the hierarchical nature of structure formation (Schnittman 2007).

Higher velocity kicks become more important if one assumes high values of pre-merger spin amplitudes: Lousto et al. (2010b) found 23\% of their Monte Carlo sample to exceed $1000 \mathrm{~km} \mathrm{~s}^{-1}$ by assuming all pre-merger spin amplitudes $a_{1}=a_{2}=0.97$ (with isotropic distribution of spin angles), and mass ratios $q$ between 1 and $1 / 16$. Spins of this magnitude are likely realistic for a nontrivial fraction of astrophysical black holes: the $\mathrm{Fe} \mathrm{K} \alpha$ line has implied a near-maximally spinning SMBH candidate (Brenneman \& Reynolds 2006). However, there is reason to believe that the spins of merging black holes may align during the inspiral phase (Bogdanović et al. 2007) in the case of gas-rich mergers. This has the potential to strongly suppress high-velocity kicks relative to an isotropic spin distribution (but see King et al. (2005) and Lodato \& Pringle (2006) for a description of how counter-alignment, which does not suppress $v_{\mathrm{k}}$ as strongly, can also occur). Dotti et al. (2010) find a dramatic reduction, with median kick velocities below $70 \mathrm{~km} \mathrm{~s}^{-1}$, although their scenario investigates the specific case where $q$ is very 


\section{CHAPTER 3. OFFSET TDES FROM RECOILED SMBHS}

near 1 , while we are concerned with a wider range of mass ratios. Their results also indicate that cooler gas accretion (adiabatic index $\gamma=7 / 5$ ) more effectively aligns spins and suppresses kicks than a warmer $(\gamma=5 / 3)$ accretion flow. More recently, it was discovered that even in gas-free mergers, relativistic spin precession is capable of aligning or anti-aligning progenitor SMBH spin vectors, and reducing median kick velocities Kesden et al. (2010). Consideration of all these factors highlights the need to generalize the work of KM08 to a more realistic distribution of kick velocities, as recoil velocities in excess of galactic escape speed are likely to be uncommon even if SMBH spins are near-maximal and unaligned.

Because the real distribution of kick velocities depends on the SMBH spin distribution and the uncertain physics of the last stages of SMBH merger, we calculate black hole trajectories given kicks of 100, 200, 300, 400,500,600, 700, 800, and $900 \mathrm{~km} \mathrm{~s}^{-1}$, and then interpolate quantities of interest such as wandering lifetime and time-averaged tidal disruption rates. We then fit these functions of $v_{\mathrm{k}}$ to plausible black hole kick distributions. In particular, we consider the three $v_{\mathrm{k}}$ distributions in Figure 2 of Lousto et al. (2010a), which assume isotropic pre-merger spins, spins aligned to within 30 degrees, and spins aligned to within 10 degrees (dry mergers, hot wet mergers, and cold wet mergers, respectively). These scenarios assume a $q$ uniformly sampled between 0 and 1. The dry merger scenario assumes spin magnitudes randomly sampled between 0 and 0.9, while the wet mergers assume spin magnitudes between 0.3 and 0.9 . In reality, the distribution of spin amplitudes also depends on factors not considered; chiefly, relativistic spin precession (Kesden et al. 2010) and chaotic versus standard accretion (Berti \& Volonteri 2008). Likewise, modeling SMBH merger histories and spin evolution using the Press-Schechter formalism indicates a top-heavy spin distribution and (at low redshift) a 
bottom heavy $q$ distribution (Volonteri et al. 2005). We leave more complicated $a_{1}, a_{2}$, and $q$ distributions to future research, although the kick distributions we consider should bracket a wide range of the available parameter space.

\subsubsection{Tidal Disruption Physics}

Stars that pass within a radius

$$
r_{\mathrm{t}}=r_{*}\left(\frac{\eta^{2} M_{\mathrm{BH}}}{m_{*}}\right)^{1 / 3}
$$

of a SMBH will be tidally disrupted. In this equation $M_{\mathrm{BH}}$ is the black hole mass, $m_{*}$ and $r_{*}$ are the stellar mass and radius, and $\eta$ is a stellar structure constant which is of order unity for main sequence stars. Detailed calculations find $\eta=0.844$ for $n=3$ polytropes, for example (Diener et al. 1995). During the disruption event, roughly one half of the stellar mass is ejected from the system, while the other half remains bound (Rees 1988) but with the change in gravitational potential across the star producing a wide spread of specific orbital energies (SQ09),

$$
\Delta \epsilon \approx \frac{3 G M_{\mathrm{BH}}}{r_{\mathrm{p}}} \frac{r_{*}}{r_{\mathrm{p}}} .
$$

Here $r_{\mathrm{p}}$ is the pericenter distance of the star on its orbit around the black hole. The bound stellar matter quickly expands to the point where hydrodynamic forces can be neglected and the gas follows roughly Keplerian trajectories, but upon return to pericenter the gas streams dissipate energy in shocks and form an accreting torus. The characteristic mass return rate is

$$
\dot{M}_{\mathrm{r}}=\frac{1}{3} \frac{m_{*}}{t_{\text {fall }}}\left(\frac{t}{t_{\text {fall }}}\right)^{-5 / 3}
$$




\section{CHAPTER 3. OFFSET TDES FROM RECOILED SMBHS}

(Phinney 1989), with the return time for the most tightly bound debris being

$$
t_{\text {fall }} \sim \frac{2 \pi}{6^{3 / 2}}\left(\frac{r_{\mathrm{p}}}{r_{*}}\right)^{3 / 2}\left(\frac{r_{\mathrm{p}}^{3}}{G M_{\mathrm{BH}}}\right)^{1 / 2}
$$

(SQ09). This simple dynamical picture has been confirmed as largely accurate by numerical hydrodynamic simulations (Evans \& Kochanek 1989). The radiative properties of the flare have been modeled by Loeb \& Ulmer (1997), Ulmer (1999), SQ09, and Lodato \& Rossi (2011). It is generally agreed that the mass infall rates can exceed the Eddington limit for days to months after disruption. This super-Eddington phase may produce an outflow with supernova-like optical luminosities. After super-Eddington infall ceases, emission is dominated by a thermal component from the accretion disk, which also photoionizes the unbound stellar debris, producing broad emission lines. Luminosity from the disk fades with a decay time of order months to years, and is peaked in the UV and soft X-ray. Upcoming transient surveys will dramatically expand the number of TDEs available for study, with the Pan-STARRS $3 \pi$ survey expected to find $\sim 10$ per year, rising to $\sim 200$ if the SQ09 predictions about super-Eddington outflows are correct. LSST is expected to find $\sim 100$ per year, or up to $\sim 6000$ if the hypothesized super-Eddington outflows are seen. In this paper we consider a black hole mass range from $10^{6} M_{\odot}$ to $10^{8} M_{\odot}$. For $M_{\mathrm{BH}} \gtrsim 10^{8} M_{\odot}$ the Schwarzschild radius of a black hole exceeds the tidal disruption radius of main sequence stars, meaning that stars will be swallowed whole rather than disrupted by non-spinning SMBHs. However, for a Kerr black hole $r_{\mathrm{t}}$ is angle-dependent, and for high values of spin, black holes as large as $\sim 7 \times 10^{8} M_{\odot}$ can still tidally disrupt main sequence stars which approach from angles near the equatorial plane (Beloborodov et al. 1992; Kesden 2012b). For simplicity we consider SMBHs up to but not above $10^{8} M_{\odot}$. 


\section{CHAPTER 3. OFFSET TDES FROM RECOILED SMBHS}

In this paper we follow the prescriptions of SQ09 and Strubbe \& Quataert (2011) for disk and super-Eddington outflow luminosity. Specifically, we model the super-Eddington outflow as a sphere with photosphere radius

$$
R_{\mathrm{ph}} \sim 10 f_{\text {out }} f_{\mathrm{v}}^{-1}\left(\frac{\dot{M}_{\mathrm{r}}}{\dot{M}_{\mathrm{Edd}}}\right) R_{p, 3 R_{\mathrm{S}}}^{1 / 2} R_{\mathrm{S}},
$$

photosphere temperature

$$
\begin{aligned}
T_{\mathrm{ph}} \sim 2 & \times 10^{5}\left(\frac{f_{\mathrm{v}}}{f_{\text {out }}}\right)^{1 / 3}\left(\frac{\dot{M}_{\mathrm{r}}}{\dot{M}_{\mathrm{Edd}}}\right)^{-5 / 12} \\
& \times M_{6}^{-1 / 4} R_{p, 3 R_{\mathrm{S}}}^{-7 / 24} \mathrm{~K}
\end{aligned}
$$

and time of peak emission equal to the larger of $t_{\mathrm{r}}$ and the time when the outer edge of the photosphere becomes optically thin,

$$
t_{\text {edge }} \sim 1 f_{\text {out }}^{3 / 8} f_{\mathrm{v}}^{-3 / 4} M_{6}^{5 / 8} R_{p, 3 R_{\mathrm{S}}}^{9 / 8} m_{*}^{3 / 8} r_{*}^{-3 / 8} \text { days. }
$$

Here the outflowing wind velocity is given by

$$
v_{\mathrm{w}}=f_{\mathrm{v}}\left(\frac{G M_{\mathrm{BH}}}{r_{\mathrm{p}}}\right)^{1 / 2},
$$

with $f_{\mathrm{v}}$ a free parameter of fiducial value 1 , and the total mass flux in the outflow is

$$
\dot{M}_{\text {out }}=f_{\text {out }} \dot{M}_{\mathrm{r}}
$$

with $f_{\text {out }}$ a parameter taken to be $0.1 . \quad \dot{M}_{\text {Edd }}$ is the SMBH's Eddington-limited mass accretion rate assuming an accretion efficiency of $0.1, M_{6}=M_{\mathrm{BH}} /\left(10^{6} M_{\odot}\right)$, $R_{\mathrm{S}}=2 G M_{\mathrm{BH}} / c^{2}$, and $R_{p, 3 R_{\mathrm{S}}}=r_{\mathrm{p}} /\left(3 R_{\mathrm{S}}\right)$.

Again following SQ09, we approximate the disk as a geometrically slim multicolor blackbody extending from the innermost stable circular orbit, $R_{\mathrm{ISCO}}$, to $2 r_{\mathrm{p}}$. Its 
temperature profile is given by

$$
\begin{aligned}
& \sigma T_{\mathrm{eff}}^{4}(R)=\frac{3 G M_{\mathrm{BH}} \dot{M}_{\mathrm{r}} f}{8 \pi R^{3}} \times \\
& \left(\frac{1}{2}+\sqrt{\frac{1}{4}+\frac{3}{2} f\left(\frac{10 \dot{M}_{\mathrm{r}} R_{\mathrm{S}}}{\dot{M}_{\mathrm{Edd}} R}\right)^{2}}\right)^{-1},
\end{aligned}
$$

with $f=1-\sqrt{R_{\mathrm{ISCO}} / R}$, and $R_{\mathrm{ISCO}}$ a function of black hole spin.

The existence of suitable emission or absorption lines for kinematic identification of a recoiling TDE is an open question. There is widespread agreement in the literature on the existence of emission lines in the immediately unbound, photoionized stellar debris (Bogdanović et al. 2004; Strubbe \& Quataert 2009, 2011). Some of these spectral features are potentially useful for distinguishing TDEs from supernovae and other transients (Kasen \& Ramirez-Ruiz 2010). Unfortunately, the velocity spread in this debris, $\Delta v \approx(2 \Delta \epsilon)^{1 / 2}$, can easily reach a large fraction of the speed of light, and details of the Doppler broadening will be determined by the inclination of the star's initial orbit around the black hole, among other unknown parameters. A more promising candidate seems to be absorption lines formed when outflowing material processes continuum radiation from the accretion disk around the black hole (Strubbe \& Quataert 2011). Most of these lines are in the UV part of the spectrum, although weaker hydrogen or helium lines may exist in optical bands, and the presence of a soft X-ray, power law tail (as has been observed in tidal flares detected by ROSAT and GALEX - see Komossa (2002); Gezari et al. (2008)) could produce absorption lines between 1 and $10 \mathrm{keV}$ as well (Strubbe \& Quataert 2011). If the wind launching speed does not vary too much in time, these lines will be sufficiently narrow (with thermal broadening $\sim 30 \mathrm{~km} \mathrm{~s}^{-1}$ ) to make the velocity difference between the outflowing wind's photosphere and the host galaxy measurable. However, the bulk outflow velocity may itself be quite large, in which case 


\section{CHAPTER 3. OFFSET TDES FROM RECOILED SMBHS}

determination of the black hole velocity would require extremely precise wind modeling. Alternatively, if $f_{\mathrm{v}} \ll 1$, a recoiling black hole's velocity could dominate the kinematic offset between the photosphere and the galaxy, and make wind launching speed variations small. A limiting case of this regime is a different model for the super-Eddington phase of mass return (Loeb \& Ulmer 1997), in which radiation pressure from the disk isotropizes returning gas streams and supports a quasi-spherical cloud of disrupted matter. In this model, measurement of the absorption offset from host galaxy lines would precisely identify which TDEs were caused by recoiling black holes. Although the validity of either of these models is at the moment unclear, recent observations of TDEs found in SDSS data (van Velzen et al. 2011b) may be more compatible with the predictions of Loeb \& Ulmer (1997). Further validation of this model (or a low $f_{\mathrm{v}}$ version of SQ09) would indicate the feasibility of kinematic identification of recoil-induced TDEs.

For a recoiling SMBH, there are two sources of stars to tidally disrupt: unbound stars encountered in its passage through the host galaxy, and the small cloud of stars in orbits that remained bound during the recoil event. Both of these sources are considered in the next subsections. For simplicity, in the remainder of this paper we set $\eta=1, m_{*}=M_{\odot}$, and $r_{*}=R_{\odot}$ (a conservative assumption - see Magorrian \& Tremaine (1999) for how generalizing to a more realistic stellar mass function can increase tidal disruption rates by a factor $\sim 2$ ).

\subsubsection{Host Galaxy Structure}

Like many of the stationary SMBH tidal disruption rate papers (Syer \& Ulmer 1999; Magorrian \& Tremaine 1999; Wang \& Merritt 2004) we consider the Nuker surface 


\section{CHAPTER 3. OFFSET TDES FROM RECOILED SMBHS}

brightness parametrization (Lauer et al. 1995)

$$
I(r)=I_{\mathrm{b}} 2^{\frac{B-\Gamma}{\alpha}}\left(\frac{r}{r_{\mathrm{b}}}\right)^{-\Gamma}\left(1+\left(\frac{r}{r_{\mathrm{b}}}\right)^{\alpha}\right)^{-\frac{B-\Gamma}{\alpha}},
$$

which was originally developed to model the surface brightnesses of nearby ellipticals and bulges resolved at the parsec level with the Hubble Space Telescope. In Equation (3.11), $r_{\mathrm{b}}$ is the break radius at which the shallow inner power law of $I(r) \propto r^{-\Gamma}$ becomes the outer power law, $I(r) \propto r^{-B}$. The strength of the break is determined by the dimensionless coefficient $\alpha$, and $I_{\mathrm{b}}$ is the surface brightness at the break radius. The profile can be Abel inverted to yield a volume density profile with power law exponents of $\gamma \approx \Gamma+1$ and $\beta \approx B+1$ when $r \ll r_{\mathrm{b}}$ and $r \gg r_{\mathrm{b}}$, respectively. The Nuker parametrization has many well-known surface brightness models as special cases (Byun et al. 1996).

The Nuker galaxies are roughly divided into two categories based on the steepness of the surface brightness profile in the centermost regions: core galaxies and cusp galaxies. It has been suggested (Merritt \& Milosavljević 2005) that core galaxies are formed when the inspiral and merger of two SMBHs ejects stars from the host galaxy's center via 3-body interactions, scouring a core from the stellar profile - though subsequent star formation can rebuild the central parsecs into a cusp in the case of gas-rich (so-called wet) mergers (Kormendy et al. 2009; Blecha et al. 2011). For this reason, we will take core galaxies as those which best represent the immediate post-recoil state of the stellar profile in gas-poor (so-called dry) mergers. We will consider cusp galaxies also, as they are likely to be relevant in wet merger scenarios. Most core galaxies in the Nuker sample tend to possess large central black hole masses $M_{\mathrm{BH}}\left(\gtrsim 10^{8} M_{\odot}\right)$, as determined by the $M_{\mathrm{BH}}-\sigma$ relation (Tremaine et al. 2002). To examine a wider range of galaxy masses, 


\section{CHAPTER 3. OFFSET TDES FROM RECOILED SMBHS}

we do not directly use observed samples of galaxies but rather create a simple mock catalog of galaxies in which we simulate the black hole trajectories. An important scaling relation which we require for all galaxies in our mock catalog is the bulge-black hole mass relation (Marconi \& Hunt 2003)

$$
M_{*}=4.06 \times 10^{10} M_{\odot}\left(\frac{M_{\mathrm{BH}}}{10^{8} M_{\odot}}\right)^{1.04},
$$

with $M_{*}$ being total stellar mass. We also fit our galaxies to empirically determined calibrations of SMBH influence radii (Merritt et al. 2009):

$$
\begin{aligned}
& r_{\text {infl }}=35 \mathrm{pc}\left(\frac{M_{\mathrm{BH}}}{10^{8} M_{\odot}}\right)^{0.56}, \\
& r_{\text {infl }}=22 \mathrm{pc}\left(\frac{M_{\mathrm{BH}}}{10^{8} M_{\odot}}\right)^{0.55} .
\end{aligned}
$$

The first of these relations holds for core galaxies and the second for cusp galaxies. Here the influence radius, $r_{\text {inf }}$ is the radius within which there is a mass in stars equal to $2 M_{\mathrm{BH}} \cdot$

The most relevant aspect of the Nuker parametrization for our purposes is the broken power-law in the stellar density profile; as we shall see, the trajectories of kicked black holes are strongly affected by central density gradients. While the most self-consistent way to proceed would be to numerically deproject scaled brightness profiles $I(r)$ into luminosity density profiles $j(r)$, we suspect this would fail to capture an important part of the physics of black hole recoil. Initially radial orbits in spherically symmetric potentials make multiple passes through galactic center, whereas initially radial orbits in nonspherical potentials will generally receive torques that increase their pericenter distance. This is of importance to this paper because orbital energy loss for recoiling black holes in dry mergers is dominated by stellar dynamical friction 


\section{CHAPTER 3. OFFSET TDES FROM RECOILED SMBHS}

during passes through the densest central regions (Gualandris \& Merritt 2008). Using a spherical potential would produce purely radial orbits, artificially shortening the wandering life time of the black hole, and decreasing the number of offset TDEs it can produce. To avoid this problem, we use an ad hoc density-potential pair that is designed to approximate the most important dynamics of a Nuker profile but which is also easily generalizable to nonspherical geometries. Specifically, we create a set of spheroidal isodensity surfaces

$$
\rho_{*}(m)= \begin{cases}K_{1} m^{-\gamma} & m<m_{\mathrm{b}} \\ K_{2} m^{-\beta} & m_{\mathrm{b}} \leq m<m_{\max } \\ 0 & m \geq m_{\max }\end{cases}
$$

using the axisymmetric pseudoradius

$$
m^{2}=\frac{r^{2}}{a^{2}}+\frac{z^{2}}{c^{2}}
$$

In these equations $r$ and $z$ are standard cylindrical coordinates, and the isodensity spheroids have dimensionless axis ratios $a$ and $c(a>c)$. The so far free parameters $\gamma, \beta$, and $m_{\mathrm{b}}$ are calibrated using the corresponding values in the spherical spatial deprojections of the most recent Nuker sample (Lauer et al. 2005). None of those three parameters vary strongly with $M_{*}$ once the core/cusp dichotomy is accounted for, so we treat them as having Gaussian distributions about their measured means, with variance also calibrated off the Lauer et al. (2005) sample. $K_{1}$ and $K_{2}$ are chosen so that the density profile is continuous at the break pseudoradius, $m_{\mathrm{b}}$, and $m_{\max }$ is selected in combination with $K_{1}, K_{2}$ to both normalize the total stellar mass, $M_{*}$, and to reproduce the correct value of $r_{\text {infl }}$. The final free parameter of our mock galaxy catalog 


\section{CHAPTER 3. OFFSET TDES FROM RECOILED SMBHS}

is ellipticity $\epsilon=1-c / a$. Fortunately for our purposes, Lauer et al. (2005) measured isophote ellipticities for the galaxies in their sample, which we use to sample ellipticity parameter space (again, due to its lack of variation with $M_{*}$, we treat ellipticity as Gaussian-distributed).

The distinction between cores and cusps deserves more consideration, however. Because SMBHs above $10^{8} M_{\odot}$ consume stars whole rather than tidally disrupting them, the majority of galaxies of interest to us fall into the mass range represented primarily by cusps. Although scaling relations for core galaxy parameters exist (Faber et al. 1997), it is not clear how reliably they can be extrapolated an order of magnitude below the smallest core galaxies in the samples which they are based on. For our mock catalog, we consider the mergers of initially cuspy galaxies, but treat separately two different limiting scenarios:

- In the first, the tendency of a binary SMBH inspiral to scour a core is outmatched by star formation, and a nuclear cusp is preserved. In this pure cusp scenario, all free parameters $\left\{\gamma, \beta, r_{\mathrm{b}}, \epsilon\right\}$ are calibrated off the sample of cusp galaxies. We assume in this case that a fraction $f_{\mathrm{g}}$ of the baryonic mass of the bulge mass is in the form of a gas disk, which we describe in more detail in $§ 2.4$.

- In the second, we consider a SMBH binary which is successful at scouring a core, either because its progenitor merger was dry or because free gas was consumed or expelled prior to the late phase of the hard binary's evolution. For this case we calibrate the constant value of $\beta$ and the initial values $\gamma_{0}, r_{\mathrm{b}, 0}$ off the cusp sample, but calibrate $\epsilon$ and final $\gamma_{\mathrm{f}}$ off the core sample, and manually "excavate" a mass deficit $\Delta M=2 M_{\mathrm{BH}}$ to determine the final break radius $r_{\mathrm{b}, \mathrm{f}}$. The final value for 
the new, cored break radius is

$$
r_{\mathrm{b}, \mathrm{f}}=\left(\frac{\frac{\Delta M}{4 \pi K_{1}\left(1-e^{2}\right)^{1 / 2}}-r_{\mathrm{b}, 0}^{3-\gamma_{0}} \frac{\beta-\gamma_{0}}{(3-\beta)\left(3-\gamma_{0}\right)}}{r_{\mathrm{b}, 0}^{\beta-\gamma_{0}} \frac{\gamma_{\mathrm{f}}-\beta}{\left(3-\gamma_{\mathrm{f}}\right)(3-\beta)}}\right)^{1 /(3-\beta)}
$$

In practice, this tends to increase the break radius by a factor of a few. Here we have used the ellipsoidal eccentricity $e=\sqrt{1-(c / a)^{2}}$. In this second scenario, we set the remnant gas fraction $f_{\mathrm{g}}=0$.

With our galaxy models fully determined, we can then integrate our density profiles numerically to calculate other relevant quantities such as the stellar potential and forces. Here we use the standard method of homoeoids (Binney \& Tremaine 2008, Section 2.5). Due to the difficulty involved in even numerical calculation of a two-integral distribution function (Hunter \& Qian 1993), particularly when an analytical, closed-form potential is lacking (as is the case here) we are forced to compute the velocity dispersion in the spherical limit and generalize by substituting $m$ for $r$; this introduces modest inaccuracy into the dynamical friction and gravitational focusing formulae used in $\S 2.4$. In the absence of other matter, the spherical limit of this broken power-law profile has a one-dimensional velocity dispersion

$$
\begin{aligned}
\sigma^{2}(r) & =2 \pi G K_{2}\left(\frac{2 r_{\mathrm{b}}^{3-\beta}}{r(3-\gamma)(1+\beta)}\right. \\
& \left.+\frac{r^{2-\beta}}{(3-\beta)(\beta-1)}-\frac{2 r_{\mathrm{b}}^{3-\beta}}{r(3-\beta)(1+\beta)}\right)
\end{aligned}
$$


for $r>r_{\mathrm{b}}$, and

$$
\begin{aligned}
\sigma^{2}(r) & =2 \pi G K_{1} r_{\mathrm{b}}^{2-2 \gamma} r^{\gamma}\left(\frac{2}{(3-\gamma)(1+\beta)}\right. \\
& +\frac{1}{(3-\beta)(\beta-1)}-\frac{2}{(3-\beta)(1+\beta)} \\
& \left.+\frac{1}{(3-\gamma)(1-\gamma)}\right)-\frac{2 \pi G K_{1} r^{2-\gamma}}{(3-\gamma)(1-\gamma)}
\end{aligned}
$$

for $r \leq r_{\mathrm{b}}$. However, the presence of a central black hole will modify $\sigma$ at small radii, while the presence of a dark matter halo (discussed below) will alter $\sigma$ at large radii. In our numerical calculations of trajectories and disruption rates, instead of the above analytic formulae we numerically compute the one-dimensional velocity dispersion (assuming isotropy) as

$$
\sigma^{2}=\frac{1}{\rho(r)} \int_{r}^{\infty} \rho\left(r^{\prime}\right) \frac{G M\left(r^{\prime}\right)}{r^{\prime 2}} \mathrm{~d} r^{\prime},
$$

where $\rho(r)$ is the total density and $M(r)$ the total mass enclosed at a radius $r$.

The stellar bulge population dominates gravitational effects for low velocity kicks, but higher velocity kicks carry SMBHs into regions where the dark matter halo potential becomes important. We model the density profile of dark matter using a standard NFW profile (Navarro et al. 1997), given by

$$
\rho_{\mathrm{NFW}}=\frac{\rho_{0}}{\left(r / a_{\mathrm{s}}\right)\left(1+r / a_{\mathrm{s}}\right)^{2}} .
$$

Here the scale distance $a_{\mathrm{s}}$ and density factor $\rho_{0}$ are determined by assuming a concentration of 10 and truncating the NFW profile at $r_{200}$, the virial radius at which $\rho_{\mathrm{NFW}}=200 \rho_{\mathrm{c}}$, with $\rho_{\mathrm{c}}$ the cosmological critical density. We also normalize the total dark matter mass $M_{\text {tot }}$ using the latest calibration of the $M_{\mathrm{BH}^{-}} M_{\text {tot }}$ relation (Bandara 
et al. 2009),

$$
M_{\mathrm{BH}}=1.51 \times 10^{8}\left(\frac{M_{\mathrm{tot}}}{10^{13} M_{\odot}}\right)^{1.55} .
$$

Our assumption of spherical symmetry for the dark matter halo should be considered conservative, since nonspherical potentials extend the wandering time of the SMBH.

Our general strategy for galaxy modeling is to select a fiducial value of SMBH mass, a parameter set $\left\{\gamma, \beta, r_{\mathrm{b}}, \epsilon\right\}$ informed by the Lauer et al. (2005) sample, and then to use the scaling relations described in this section to self-consistently find other parameters so that integrated quantities like potential or velocity dispersion can be numerically computed. The fiducial values of $M_{\mathrm{BH}}$ we use are $10^{6}, 10^{6.5}, 10^{7}, 10^{7.5}$, and $10^{8} M_{\odot}$. To parallel the SQ09 calculation we also use the black hole mass function inferred by Hopkins et al. (2007).

In minor mergers, the dynamical friction timescale for satellite infall is expected to be greater than the Hubble time (Wetzel \& White 2010), meaning that we only need to consider the mass range of $q=0.1-1$. Simulated merger rates for this mass ratio range in galaxies with stellar mass $M_{*}>M_{\min }$ have been matched (Hopkins et al. 2010) to the analytic fit

$$
\begin{aligned}
\frac{d N_{\text {major }}}{d t} & =0.04\left(1+\left(\frac{M_{\min }}{M_{0}}\right)^{0.8}\right) \\
& \times(1+z)^{\beta\left(M_{\min }\right)} \mathrm{Gyr}^{-1},
\end{aligned}
$$

with $z$ the redshift of the galaxies, $M_{0}=2 \times 10^{10} M_{\odot}$, and

$$
\beta\left(M_{\min }\right)=1.5-0.25 \log \left(\frac{M_{\min }}{M_{0}}\right) .
$$

We use this merger rate in combination with the volumetric SMBH mass function and the results of our orbit integrations (described below) to compute the total rate of events 
observable by LSST. For all calculations in this paper we assume a standard $\Lambda \mathrm{CDM}$ cosmology with $\Omega_{\Lambda}=0.73, \Omega_{\mathrm{M}}=0.27$, and $H_{0}=71 \mathrm{~km} \mathrm{~s}^{-1} \mathrm{Mpc}^{-1}$ (Spergel et al. 2007).

\subsubsection{Interactions with the Galaxy}

To model the disruption rate of unbound stars, we evolve the SMBH's trajectory under the influence of gravity and dynamical friction through galaxies with the axisymmetric Nuker density profiles described above. We use the fifth-order Dormand-Prince method (with an embedded fourth-order Runge-Kutta integrator for adaptive timestepping) described in Press et al. (2002, Chapter 17). The effects of dynamical friction are approximated with the Chandrasekhar formula (Chandrasekhar 1943),

$$
\vec{F}_{\mathrm{df}}=-I(M) \frac{4 \pi \rho\left(G M_{\mathrm{BH}}\right)^{2}}{\sigma^{2}} \frac{\overrightarrow{\mathrm{v}}_{\mathrm{BH}}}{v_{\mathrm{BH}}},
$$

with $\sigma$ the local velocity dispersion and $\rho$ the local density of the medium causing the drag. For a collisionless medium, such as a stellar population,

$$
I_{\text {dry }}(M)=\frac{\ln (\Lambda)}{M^{2}}\left(\operatorname{erf}\left(\frac{M}{\sqrt{2}}\right)-\sqrt{\frac{2}{\pi}} M e^{-M^{2} / 2}\right)
$$

where $M=v_{\mathrm{BH}} / \sigma$ is the Mach number. The Coulomb logarithm can be fit numerically (Escala et al. 2004), and for the case of SMBHs on radial orbits, is well matched by a value of $\ln \Lambda=2.5$ (Gualandris \& Merritt 2008). Dynamical friction is the force which ultimately causes the kicked black hole to settle back into a near-stationary position in the center of its host galaxy, on timescales ranging from $10^{6}$ to $10^{9}$ years (Madau \& Quataert 2004; Blecha \& Loeb 2008). The Chandrasekhar formula is derived assuming a uniform and infinite background of stars, and it is not immediately clear how appropriate that is for a steep density profile in galactic nuclei, or for a black hole massive enough to excite a response in the stellar population. The applicability of the Chandrasekhar 


\section{CHAPTER 3. OFFSET TDES FROM RECOILED SMBHS}

formula to bound, recoiling black holes has been considered before (Gualandris \& Merritt 2008), and for appropriately chosen $\ln \Lambda$ it was found to be fairly accurate until the point when the mass interior to the black hole's apogalacticon is of order $M_{\mathrm{BH}}$. After this, coherent oscillations develop in the stars interior to the black hole's trajectory, and dynamical friction is found to become dramatically less effective at removing the black hole's orbital energy. We terminate our calculations at the onset of this orbital phase, both because our trajectory approximation would become quite inaccurate and also because TDEs caused by a slow-moving SMBH near the center of a galaxy would not be distinguishable from those caused by a stationary black hole. During these calculations, we neglect the extra "core scouring" caused by black hole recoil (Gualandris \& Merritt 2008). The stellar population in the galactic center responds to a moving SMBH by expanding, with a mass of stars equal to a few $M_{\mathrm{BH}}$ being displaced from the galactic center for kicks close to escape velocity (and the effect is reduced for slower ones). Neglect of this effect likely reduces the SMBH wandering time and causes us to underestimate the total number of TDEs per galaxy merger, but probably not by much, as axisymmetry of the stellar potentials prevents the SMBHs from returning exactly to the center of their host galaxies where core scouring is most relevant. We highlight that dynamical friction removes the most orbital energy during passages through the densest regions of the SMBH's trajectory. Therefore trajectories with nonzero angular momentum (due to an axisymmetric potential) last longer before settling back into the galactic center than would center-crossing ones in spherical geometries.

In a dry merger it is sufficient to consider dynamical friction off stars and not gas. This regime could also apply to wet mergers where the gas is used up in star formation (while the SMBH binary is stalled) or dispersed in binary quasar feedback. We identify 


\section{CHAPTER 3. OFFSET TDES FROM RECOILED SMBHS}

both of these scenarios with our "excavated core" galaxies. If significant quantities of gas survive until the recoil phase of the merger, however, it is necessary to consider the effects of gas dynamical friction, which would apply more to our "pure cusps." Previous work (Blecha et al. 2011) has indicated the effect of leftover gas is to decrease black hole wandering times, reducing the observable number of offset TDEs. To quantify this effect, Equation (3.26) still applies; we simply need to use gas density rather than stellar density for $\rho$, substitute a local sound speed $c_{\mathrm{s}}$ for $\sigma$, and modify the dimensionless parameter $I(M)$ (for gas, $M=v_{\mathrm{BH}} / c_{\mathrm{s}}$ ). The new dimensionless functions are

$$
I_{\text {subsonic }}(M)=\frac{1}{2} \ln \left(\frac{1+M}{1-M}\right)-M
$$

in the subsonic regime, and

$$
I_{\text {supersonic }}(M)=\frac{1}{2} \ln \left(1-\frac{1}{M^{2}}\right)+\ln \left(\frac{v_{\mathrm{BH}} t}{r_{\min }}\right)
$$

in the supersonic regime (Ostriker 1999). However, these formulae have been shown to overestimate gas dynamical friction in the slightly supersonic regime, so we adopt the prescription of Escala et al. (2004) and use the Chandrasekhar formula for $I(M)$, with $\ln \Lambda=4.7$ for $M \geq 0.8$ and $\ln \Lambda=1.5$ for $M<0.8$. We follow the prescriptions of Blecha \& Loeb (2008) and assume that most of the gas in the galaxy has settled into a disk, which we align with the oblate plane of the galaxy. We employ a slightly less complicated version of their model, as only two of their four disk zones are relevant for our dynamical modeling: zones III and IV (zones I and II only exist in the presence of a central SMBH). Zone III, the portion of the disk influenced by the SMBH potential before the recoil kick, is truncated on its inner edge at the kick radius,

$$
r_{\mathrm{k}}=\frac{G M_{\mathrm{BH}}}{v_{\mathrm{k}}^{2}},
$$

and transitions to zone IV at $r=r_{\text {infl. }}$ Zone IV is an exponential disk with scale $r_{\text {disk }}$. 
The disk surface density in zone III will be

$$
\Sigma_{\text {III }}=\left(\frac{4}{\pi Q^{2}}\right)\left(\frac{\dot{M}_{\alpha}^{2}}{\alpha^{2} G}\right)^{1 / 3} r^{-1},
$$

while in zone IV, the surface density is

$$
\Sigma_{\mathrm{IV}}=\frac{M_{\text {disk }}\left(>r_{\text {inff }}\right)}{2 \pi r_{\text {disk }}\left(r_{\text {infl }}+r_{\text {disk }}\right) e^{-r_{\text {inf }} / r_{\text {disk }}}} e^{-r / r_{\text {disk }}} .
$$

Here we take the viscosity parameter $\alpha=0.1$, and set the Toomre parameter $Q=1$ (Toomre 1964) under the assumption that star formation feedback roughly balances cooling, leaving the disk marginally stable. The accretion rate $\dot{M}_{\alpha}$ can be found by mass normalization of equation (3.30) so that $M_{\mathrm{III}}=2 f_{\mathrm{gas}} M_{\mathrm{BH}}$ :

$$
\dot{M}_{\alpha}=\left(\frac{f_{\mathrm{gas}} M_{\mathrm{BH}} Q^{2}}{4\left(r_{\mathrm{infl}}-r_{\mathrm{k}}\right)}\right)^{3 / 2} \alpha G^{1 / 2} .
$$

The scale distance $r_{\text {disk }}$ is then found by requiring continuity between zones III and IV: $\Sigma_{\mathrm{III}}\left(r_{\text {inff }}\right)=\Sigma_{\mathrm{IV}}\left(r_{\text {infl }}\right)$. Densities in both disk zones decay exponentially with height $z$, with scale height

$$
h_{\mathrm{III}}=\frac{Q^{2}}{8} r
$$

taken from Blecha \& Loeb (2008). In zone IV, the scale height

$$
h_{\mathrm{IV}}=\frac{\dot{M}_{\alpha} \kappa_{\Omega}}{3 \pi^{2} \alpha Q G \Sigma_{\mathrm{IV}}^{2}}
$$

is solved for using the identities $\dot{M}_{\alpha}=3 \pi \alpha c_{\mathrm{s}} h \Sigma$ and $c_{\mathrm{s}}=\left(G \dot{M}_{\alpha} / \alpha\right)^{1 / 3}$. Here $\kappa_{\Omega}$ is the epicyclic frequency, and is calculated from the numerically integrated potentials of the isodensity shells in Equation (3.15).

In our models we consider values of $f_{\mathrm{g}}$ (gas as a fraction of total baryonic mass) of 0 and 0.3. The latter value is taken as a conservative upper limit for remnant gas fraction at the time of black hole merger, as self-consistent hydrodynamical simulations (Mihos \& Hernquist 1996) have shown that $\geq 50 \%$ of the initial gas fraction, $f_{\mathrm{g}, \mathrm{i}}$ in a merger 
is expelled or converted into stars by the time of black hole coalescence. Observation indicates that $f_{\mathrm{g}, \mathrm{i}} \lesssim 0.6$ for $M_{\mathrm{BH}}>10^{6} M_{\odot}$ at low redshift (Hopkins et al. 2010, Figure 7), so $f_{\mathrm{g}}=0.3$ is a conservative case, likely to result in SMBH orbits which decay somewhat faster and produce fewer offset tidal disruptions than in more general wet mergers with smaller $f_{\mathrm{g}}$.

At each point along the SMBH's trajectory we consider an instantaneous "tidal disruption cylinder" of length $v_{\mathrm{BH}} \Delta t$ and radius equal to the gravitationally focused tidal disruption radius. This lets us simply calculate instantaneous tidal disruption rates along the trajectory,

$$
\dot{N}_{\mathrm{u}}=\rho_{*} v \pi r_{\mathrm{t}}^{2}\left(1+\frac{2 G M_{\mathrm{BH}}}{r_{\mathrm{t}} v^{2}}\right)
$$

which can be integrated to get a time-averaged TDE rate, or $N_{\text {TDE }}$, the total number of

stars disrupted per recoil event. Here $v=\sqrt{v_{\mathrm{BH}}^{2}+\sigma^{2}}$, with $\sigma$ given by Equations (3.18) and (3.19).

\subsubsection{Interactions with the Bound Cloud}

The initial size of the bound cloud is determined by the magnitude of the received kick, and can be approximated as encompassing all stars within $r_{\mathrm{k}}$. The mass of the bound cloud is found by KM08 to be a fraction $f_{b}$ of the black hole mass, where

$$
f_{b}=F(\gamma)\left(\frac{2 G M_{\mathrm{BH}}}{r_{\mathrm{inff}} v_{\mathrm{k}}^{2}}\right)^{3-\gamma} .
$$

Here $\gamma$ is the same as in Equation (3.15), $r_{\text {inf }}$ is the influence radius, the interior of which contains a mass in stars twice $M_{\mathrm{BH}}$, and $F(\gamma)=11.6 \gamma^{-1.75}$. For most cloud sizes the disruption rate of bound stars will be determined by resonant relaxation into the 


\section{CHAPTER 3. OFFSET TDES FROM RECOILED SMBHS}

SMBH's empty loss cone, exponentially depleting the population of stars inside on a timescale $\tau \approx 3.6 G M_{\mathrm{BH}}^{2} /\left(v_{\mathrm{k}}^{3} m_{*}\right)$ (KM08). In practice, the e-folding time is at least an order of magnitude below $10^{10}$ years for most of the $10^{6} M_{\odot}$ and $10^{6.5} M_{\odot}$ black holes which escape from their host galaxies, strongly suppressing the averaged intergalactic TDE rate.

One exception to this picture is if $r_{\mathrm{k}} \sim r_{\mathrm{inf}}$; in this case nonresonant relaxation could become important, and resonant relaxation alone will significantly underestimate the TDE rate. This regime is of minimal significance for this paper, however, since small kicks are likely to produce few spectrally and no spatially offset flares. A more significant exception is for relatively low-mass SMBHs, which can reach an energy relaxation timescale in less than their wandering time. Relaxation will eventually allow the cloud to expand in radius (O'Leary \& Loeb 2009), changing the time evolution of the tidal disruption rate from exponential depletion to $\propto t^{-3 / 2}$ (O'Leary \& Loeb 2012). Therefore, we adopt KM08's prescription for resonant relaxation,

$$
\dot{N}_{b} \approx C_{\mathrm{RR}}(\gamma) \frac{\ln \Lambda}{\ln \left(r_{\mathrm{k}} / r_{\mathrm{t}}\right)} \frac{v_{\mathrm{k}}}{r_{\mathrm{k}}} f_{\mathrm{b}} e^{-t / \tau}
$$

when $t<t_{\mathrm{r}}$, but transition to $\dot{N}_{\mathrm{b}} \propto t^{-3 / 2}$ at later times. The energy relaxation timescale $t_{\mathrm{r}}$ is taken to be (O'Leary \& Loeb 2012)

$$
t_{\mathrm{r}}=10^{9} \operatorname{yrs}\left(\frac{M_{\mathrm{BH}}}{10^{5} M_{\odot}}\right)^{5 / 4}\left(\frac{r_{\mathrm{k}}}{r_{\text {infl }}}\right)^{1 / 4} .
$$

This power law disruption rate is only relevant for $M_{\mathrm{BH}}<10^{6.5} M_{\odot}$, but for lower mass SMBHs we transition to power law depletion after an energy relaxation time. For both 
scenarios, the initial disruption rate is

$$
\begin{aligned}
\dot{N}_{b} \approx 1.5 \times & 10^{-6}\left(\frac{M_{\mathrm{BH}}}{10^{7} M_{\odot}}\right)\left(\frac{r_{\mathrm{infl}}}{10 \mathrm{pc}}\right)^{-2} \\
& \times\left(\frac{v_{\mathrm{k}}}{10^{3} \mathrm{~km} \mathrm{~s}^{-1}}\right)^{-1} \mathrm{yr}^{-1}
\end{aligned}
$$

One uncertainty is the resonant relaxation coefficient $C_{\mathrm{RR}}$, found by KM08 to have a value of 0.14 for $\gamma=1$. Since the spatial power law exponents for core galaxies are close to 1 , we adopt this value, though it is less well motivated for cuspier galaxies.

We also consider growth of the bound cloud by capture of members of binary star systems. This three-body interaction is treated in the same way as tidal disruption of unbound stars, except instead of a stellar tidal disruption radius we use an "orbital tidal disruption radius", given by

$$
r_{\mathrm{t}, \mathrm{o}}=a_{\mathrm{bin}}\left(\frac{M_{\mathrm{BH}}}{2 m_{*}}\right)^{1 / 3},
$$

where $a_{\text {bin }}$ is the binary semimajor axis. While one member of the binary is ejected at high velocities (Hills 1988), the other is bound to the black hole, with apoapsis $r_{\max }$ given by

$$
r_{\max } \approx \frac{G M_{\mathrm{BH}}}{v_{\text {eject }}^{2}}\left(\frac{m_{*}}{M_{\mathrm{BH}}}\right)^{1 / 6}\left(\frac{a}{0.1 \mathrm{AU}}\right)^{1 / 2}
$$

(Yu \& Tremaine 2003), with $v_{\text {eject }} \approx 145 \mathrm{~km} \mathrm{~s}^{-1}$ (Hills 1988). To calculate the rate of these captures, we assume Öpik's Law (Öpik 1924), a flat distribution of binary semimajor axes $a$ in units of $\log (a)$, between $a_{\min }$ and $a_{\max }$. Following Vereshchagin et al. (1988) and Kouwenhoven et al. (2007) we adopt $a_{\min }=5 R_{\odot}$ and $a_{\max }=5 \times 10^{6} R_{\odot}$. We only consider captures with $r_{\max }<r_{\mathrm{t}, \mathrm{c}}$, with the cloud's tidal radius conservatively given by $r_{\mathrm{t}, \mathrm{c}}=r_{\mathrm{infl}}$. This refill mechanism is in principle capable of counterbalancing losses due to tidal disruption and evaporation from the cloud. Without a refill source, resonant 


\section{CHAPTER 3. OFFSET TDES FROM RECOILED SMBHS}

relaxation into the loss cone will normally cause the population of the bound cloud to evolve due to $\dot{N}_{\text {TDE }} \propto N$, leading to a population (and TDE rate) depleted exponentially in time, at least until $t=t_{\mathrm{r}}$. We can roughly see the effect of stellar capture into the bound cloud if we assume

$$
\dot{N}=-k N+m
$$

with $k$ the average frequency with which bound stars evaporate or are scattered into the loss cone and $m$ a time-averaged capture rate. This differential equation has the solution

$$
N(t)=N(0) e^{-k t}+\frac{m}{k}\left(1-e^{-k t}\right) .
$$

By itself, relaxation will deplete the bound cloud, but 3-body capture allows the number of stars in the cloud to asymptotically approach a nonzero value. If the time-averaged binary capture rate is high enough (i.e. if $m / k>N(0)$ ) the size of the cloud would even grow over time. The importance of this effect is determined for each galaxy/kick velocity pair.

A final consideration is stability of the bound cloud to perturbations. Analytically, it seems unlikely that interactions with unbound stars will eject significant numbers of bound stars from the cloud: if the cloud stars are bound to the black hole with typical energy $E_{\text {bind }} \sim-v_{\mathrm{k}}^{2} m_{*}$, and during encounters with unbound stars a change in energy $\Delta E \sim G m_{*}^{2} / r_{\mathrm{p}}$ is available (where $r_{\mathrm{p}}$ is the closest approach of the two stars), encounters must be within $r<r_{\mathrm{p}} \sim r_{\mathrm{k}}\left(m_{*} / M_{\mathrm{BH}}\right)$. For a $10^{7} M_{\odot}$ black hole on typical trajectories, this works out to at most $\sim 1$ unbound stars making close enough approaches to eject a bound star during the SMBH's passages through the bulge. 


\subsubsection{Observability of Recoil-Induced TDEs}

To translate the total recoil-induced TDE rate into a rate of identifiably recoil-induced TDEs, it is necessary to consider observational constraints. LSST's rapid cadence, high sensitivity and thorough sky coverage make it an ideal survey to detect disruption flares - as mentioned in $\S 2.2$, LSST could detect up to thousands of TDEs per year. LSST's limiting g-band magnitude is 25 (LSST Science Collaboration et al. 2009); because of LSST's short cadence we assume any flares brighter than that will be detected. The detectability of a spatial offset will depend on how well the TDE centroid can be distinguished from the host galaxy centroid after photometric frame subtraction. For LSST the expected differential astrometric precision will be $\sim 0.7^{\prime \prime} / \mathrm{SNR}$ (LSST Science Collaboration et al. 2009). Using the LSST Science Manual's prescription for $\mathrm{SNR}^{-1}=\sigma_{\mathrm{tot}}=\sqrt{\sigma_{\mathrm{sys}}^{2}+\sigma_{\text {rand }}^{2}}$, we infer astrometric precision by calculating the signal to noise ratio for each event in our sample. We also calculate the rate of spectroscopically identifiable flares associated with a recoiling SMBH. Although UV spectroscopy would be ideal, soft X-ray spectrometers - SXS, for example, on the planned ASTRO-H mission (Takahashi et al. 2010), expected to be operating contemporarily with LSST - should be able to identify the absorption lines discussed in $\S 2.2$, if they exist with sufficient equivalent width. To investigate this possibility, we consider a fiducial case of absorption lines at $10 \mathrm{keV}$, observed by SXS followup with an energy resolution of $7 \mathrm{eV}$. If the outflowing wind can be accurately modeled, these lines would allow black hole velocities down to $\sim 200 \mathrm{~km} \mathrm{~s}^{-1}$ to be spectrally resolvable. As mentioned before, it is not clear that the super-Eddington phase of accretion will produce winds in which a $\sim 200 \mathrm{~km} \mathrm{~s}^{-1}$ offset is detectable, so predictions of kinematic offsets should be regarded as somewhat hypothetical. Because spatial and kinematic offsets are angle-dependent, we average the 
observable quantities over all inclination angles for the host galaxy.

\subsection{TDE Rate}

Using the potentials and frictional forces described above, we integrate the trajectories of five different black hole masses at nine different kick velocities and seven inclination angles in galaxies with eighteen different possible permutations of mass-independent structural parameters, for a total of 11340 runs (the final factor of two comes from wet vs dry mergers). During preliminary test runs, a very weak dependence of the wandering time on $\beta$ and $\gamma$ was apparent (once variation in $\beta$ and $\gamma$ due to the core/cusp dichotomy is allowed for), so we set those quantities equal to their average values. Among the remaining structural parameters, we only varied $\epsilon$ and $r_{\mathrm{b}, 0}$.

We terminate our trajectory calculations after a Hubble time, if the black hole has left the stellar bulge (and its attendant sources of friction) with escape velocity, or upon the onset of the "Phase II" orbital oscillations of Gualandris \& Merritt (2008), discussed in $\S 2.4$.

To calculate the total observable rate of TDEs due to recoiling black holes, $\Upsilon$, we use a modified version of Equation (31) in SQ09. Specifically,

$$
\begin{aligned}
\frac{\mathrm{d} \Upsilon}{\mathrm{d} \ln M_{\mathrm{BH}}} & =\int_{r_{\mathrm{ISCO}}}^{r_{\mathrm{t}}} \int_{0}^{\mathrm{d}_{\max }\left(r_{\mathrm{p}}\right)} 4 \pi r^{2} f_{\text {sky }} \frac{\mathrm{d} n}{\mathrm{~d} \ln M_{\mathrm{BH}}} \\
& \times \frac{\mathrm{d} v\left(r, r_{\mathrm{p}}\right)}{\mathrm{d} \ln r_{\mathrm{p}}} \mathrm{d} r \mathrm{~d} \ln r_{\mathrm{p}}
\end{aligned}
$$

Here $d_{\max }\left(r_{\mathrm{p}}\right)$ represents the maximum comoving distance a TDE flare with given pericenter $r_{\mathrm{p}}$ can be seen at, using a $25 \mathrm{AB}$ g-band magnitude limit. LSST will detect flares at cosmological distances, so it is necessary to employ a K-correction, which has a 


\section{CHAPTER 3. OFFSET TDES FROM RECOILED SMBHS}

modest impact on $d_{\max }$. The rate $v\left(r, r_{\mathrm{p}}\right)$ is integrated over inclination/azimuth angles and galaxy properties, and is the rate at which either TDE flares are produced at a distance $r$ by TDEs with $r_{\mathrm{p}}$. Further cuts are added to the integrand to calculate the rate at which observably spatially offset TDE flares, or observably kinematically offset TDE flares are produced, using the criteria described in $\S 2.6$ (with an average over azimuthal angles to account for projection effects). In our average over galaxy properties, we give $M_{\mathrm{BH}}$-dependent weights to the "pure cusp" and "excavated core" scenarios from §2.3. These weights are determined in two ways. The first is to bin the the Lauer et al. (2005) galaxy sample and compute the fraction of cusps and cores in each $\sigma$ bin (with the small minority of intermediate cases taken as $50 \%$ core, $50 \%$ cusp). The $\sigma$ bins are translated into masses from the measured $\sigma$ using a recent calibration (Graham et al. 2011) of the $M_{\mathrm{BH}}-\sigma$ relation (Tremaine et al. 2002). The second approach is to bin the larger Lauer et al. (2007) sample in magnitude $M_{\mathrm{V}}$, and to then translate to $M_{\mathrm{BH}}$ using the relation between $M_{\mathrm{BH}}$ and V-band magnitude in Bentz et al. (2009) (specifically, the "FITEXY FF05 ellipticals - outliers" fit). We average the results of these two methods, and find that for $M_{\mathrm{BH}} / M_{\odot}$ of $10^{6}, 10^{6.5}, 10^{7}, 10^{7.5}$, and $10^{8}$, the fractions of core galaxies are $0.03,0.125,0.125,0.114$, and 0.302 , respectively.

To calculate $d_{\max }$ for super-Eddington flares we use Equations (3.5), (3.6), and (3.7), while to do the same for disk emission we use Equation (3.10). For simplicity, we neglect the less important emission from photoionized, unbound disruption debris, noting that this is a conservative approximation. For disruptions from the bound cloud, resonant relaxation slowly diffuses stars across the loss cone in phase space, meaning that nearly all bound TDEs will have $r_{\mathrm{p}} \approx r_{\mathrm{t}}$. Unbound stars will have a wider variety of $r_{\mathrm{p}}$, but the geometry of gravitational focusing will bias them towards $r_{\mathrm{p}} \approx r_{\mathrm{t}}$ as well. 


\section{CHAPTER 3. OFFSET TDES FROM RECOILED SMBHS}

For these reasons we simplify Equation (3.44) by taking $r_{\mathrm{p}}=r_{\mathrm{t}}$. This approximation produces slightly more disk emission (due to physically larger disks), and significantly less luminous super-Eddington flares, than does the flat distribution of TDEs across $\ln r_{\mathrm{p}}$ assumed in SQ09. Consequently, our results show a much less pronounced difference in the observable TDE rate between the disk emission and super-Eddington outflows cases.

We then interpolate the results of these trajectory calculations over three different black hole physics scenarios, as discussed in $\S 2.1$. In the first scenario, a lack of free gas during the SMBH inspiral leaves the spin vectors of the SMBH binary randomly aligned with each other, producing a top-heavy kick distribution and a high average value of dimensionless spin $(a=0.73)$. The other two scenarios involve wet mergers with warmer and cooler gas, producing spin vectors aligned to within 30 and 10 degrees, respectively, and remnant mean spins of $a=0.88$ and $a=0.90$. Because the disruption of stars by $10^{8} M_{\odot}$ black holes is so sensitive to $a$ ( $a>0.92$ required), we bracket these fiducial assumptions (the $a$ values above are the peaks of the remnant spin probability distributions in Lousto et al. (2010a)) about remnant spin with $a=0$ and $a=0.99$ cases. We also consider two different cases of tidal disruption physics; one in which the super-Eddington mass outflows proposed by SQ09 exist (for simplicity we take their canonical case of $f_{\mathrm{v}}=1$ and $f_{\text {out }}=0.1$ ), and the other in which they do not. In the latter, optical emission is limited to the Rayleigh-Jeans tail of the newly-formed accretion disk. 


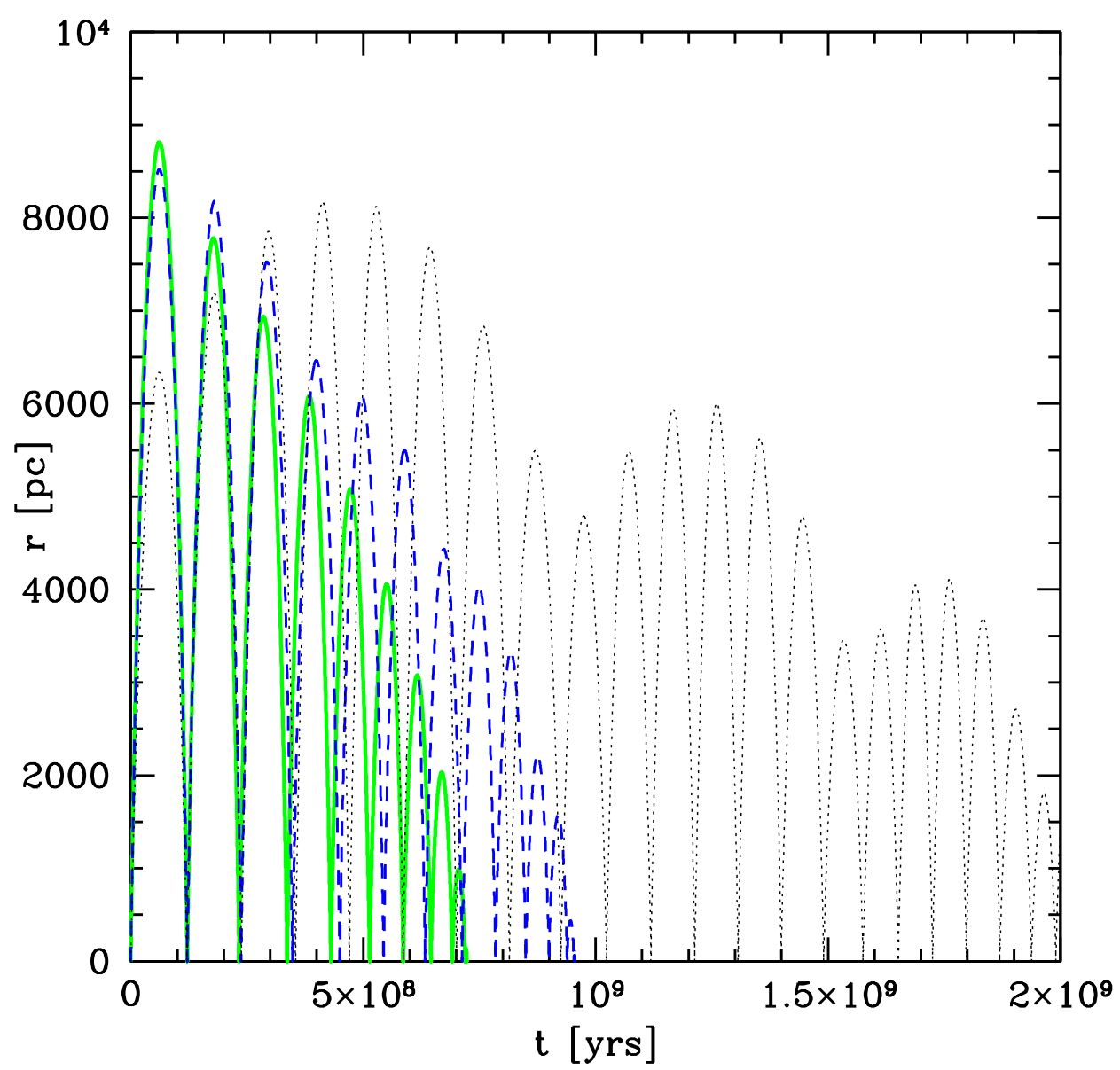

Figure 3.1.- : The importance of nonsphericity on SMBH trajectories. All the lines represent trajectories of $10^{7} M_{\odot}$ SMBHs kicked at $400 \mathrm{~km} \mathrm{~s}^{-1}$ in a gas-free core galaxy. The green solid line is the course of a SMBH kicked in the plane of the galaxy's two semimajor axes, while the blue dot-dashed line represents a SMBH kicked 15 degrees above the plane, and the black dotted line a SMBH kicked 45 degrees above the plane. 


\subsection{Results and Discussion}

Figures 3.1 and 3.2 illustrate the importance of nonspherical potentials for the lifetimes of wandering black holes. In the axisymmetric stellar potential that we employ, stars initially on radial orbits will quickly acquire angular momentum unless they lie on a principal axis of the stellar ellipsoid or in the equatorial plane. The latter is true of the $0^{\circ}$, green orbit, which is seen in Figure 1 to decay somewhat faster than the blue, $15^{\circ}$ orbit and dramatically faster than the $45^{\circ}$, black orbit. For the dry mergers illustrated here, the variation in decay time is due entirely to differences in stellar dynamical friction, which is the strongest at orbital pericenter. In the wet merger scenarios we considered, recoils in the plane of the gas disk are very quickly damped out, but axisymmetry in the stellar potential still affects orbital lifetimes for other inclination angles. Figure 3.2 illustrates the torques that act on orbits out of the equatorial plane, and in the inset we can see that those torqued orbits are able to avoid close pericenter passages, explaining their longevity.

The large density variations across the SMBH host galaxies can be seen in the unbound stellar disruption rate, displayed in Figure 3.3, which spans many orders of magnitude. The spikes are located at passages through the galactic center, and their increasing magnitude with each cycle arises from the inverse relation between gravitationally focused cross section and velocity. The thick lines, representing the disruption rate of bound stars, are much more constant in time, although for lower-mass SMBHs these become observably depleted, as discussed earlier. In Figure 3.4, we plot the unbound stellar disruption rate versus radial distance, and can clearly see the break in the stellar density profile near 7 pc. Scatter in Figure 3.4 is due to the combination of 


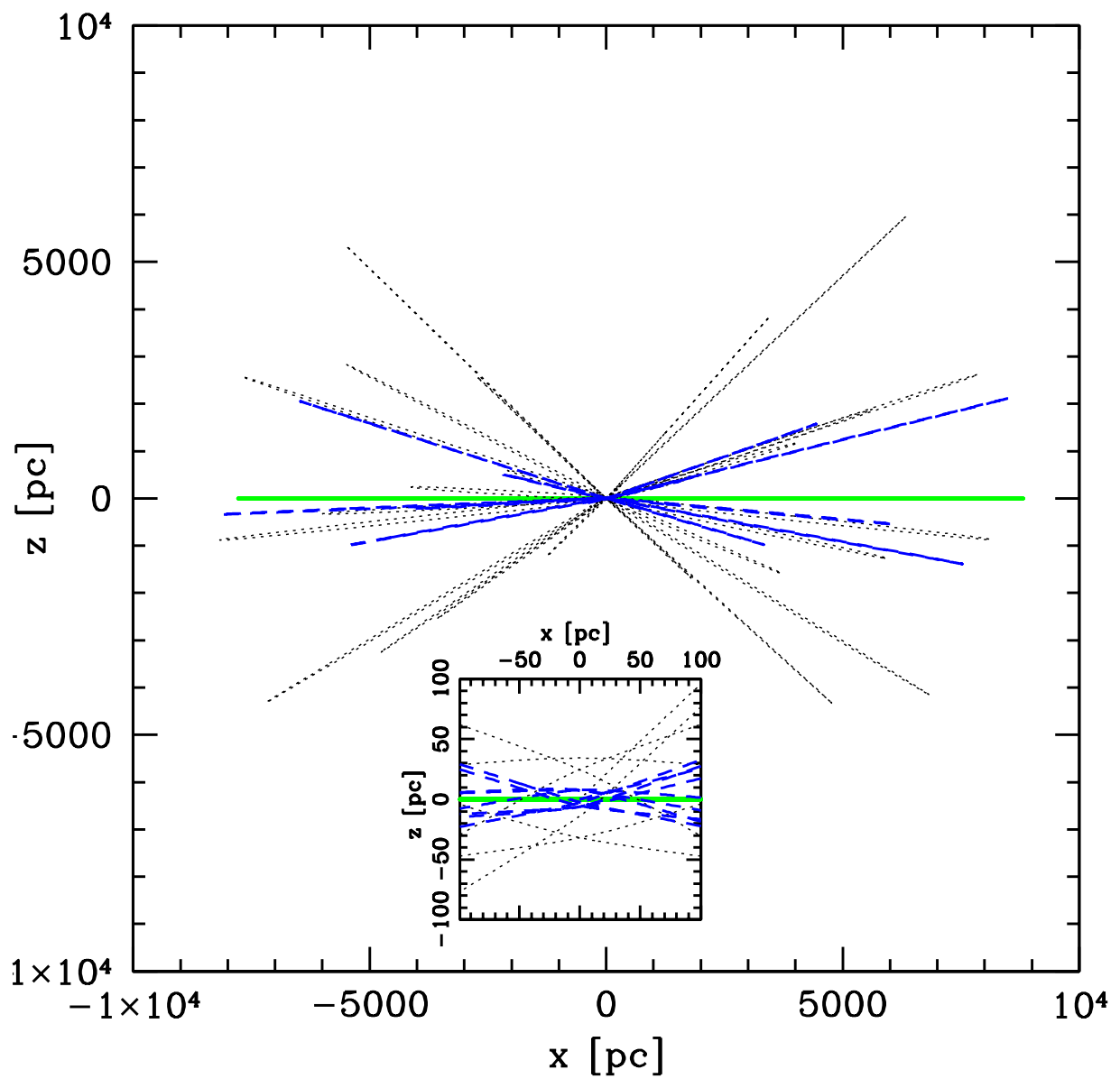

Figure 3.2.- : The same black holes as in Figure 1, now viewed in two dimensions. The inset plot zooms in on the central 100 parsecs to highlight the lack of center crossings for SMBHs ejected at nonzero inclination angles. 


\section{CHAPTER 3. OFFSET TDES FROM RECOILED SMBHS}

different velocities and different inclination angles during pericenter passage. The binary capture effect hypothesized in $\S 2.5$ was seen at low levels but found not to contribute substantially to bound cloud sizes or disruption rates.

The total number of disruptions per merger, $N_{\mathrm{TDE}}$, was found to be fairly insensitive to the power-law slopes $\gamma$ and $\beta$, but $1 \sigma$ changes in $r_{\mathrm{b}}$ or $\epsilon$ can change $N_{\text {TDE }}$ by a factor of a few. The sensitivity to $r_{\mathrm{b}}$ really reflects a sensitivity to the ratio $v_{\mathrm{k}} / v_{\mathrm{esc}}$, as the wandering time can jump by $\sim 1-2$ orders of magnitude when $v_{\mathrm{k}} / v_{\text {esc }}$ rises above a value $\sim 0.5-0.6$ (where $v_{\text {esc }}$ here is the escape velocity of the stellar bulge). This sensitivity to kick velocity is due to the decreased effectiveness of dynamical friction once the SMBH begins passing through the galactic center at high velocities, giving the black hole more time to disrupt bound cloud stars. Because the SMBHs spend most of their near-radial orbit at apocenter, most bound cloud disruptions occur at an observably offset distance.

Figs. 3.5 and 3.6 illustrate how our results vary with assumptions about the kick velocity distribution, final spin amplitudes of the SMBHs, and existence of super-Eddington outflows. In both figures, bound cloud disruptions are represented as thick lines and unbound stellar disruptions as thin lines. The total number of disruptions is shown as a solid line, while those with an observable spatial offset are shown with a dotted line, and those with an observable kinematic offset are shown with a dashed line. Unless otherwise noted, discussion of TDE rates in this section refers only to SMBHs which remain bound to their host galaxy.

Figure 3.5 displays $\mathrm{d} \Upsilon / \mathrm{d} \ln M_{\mathrm{BH}}$, the number of TDEs observed by LSST per year per logarithmic black hole mass, for our models without super-Eddington emission. Both the unaligned $\left(<180^{\circ}\right)$ and moderately aligned $\left(<30^{\circ}\right)$ progenitor spin models produce 


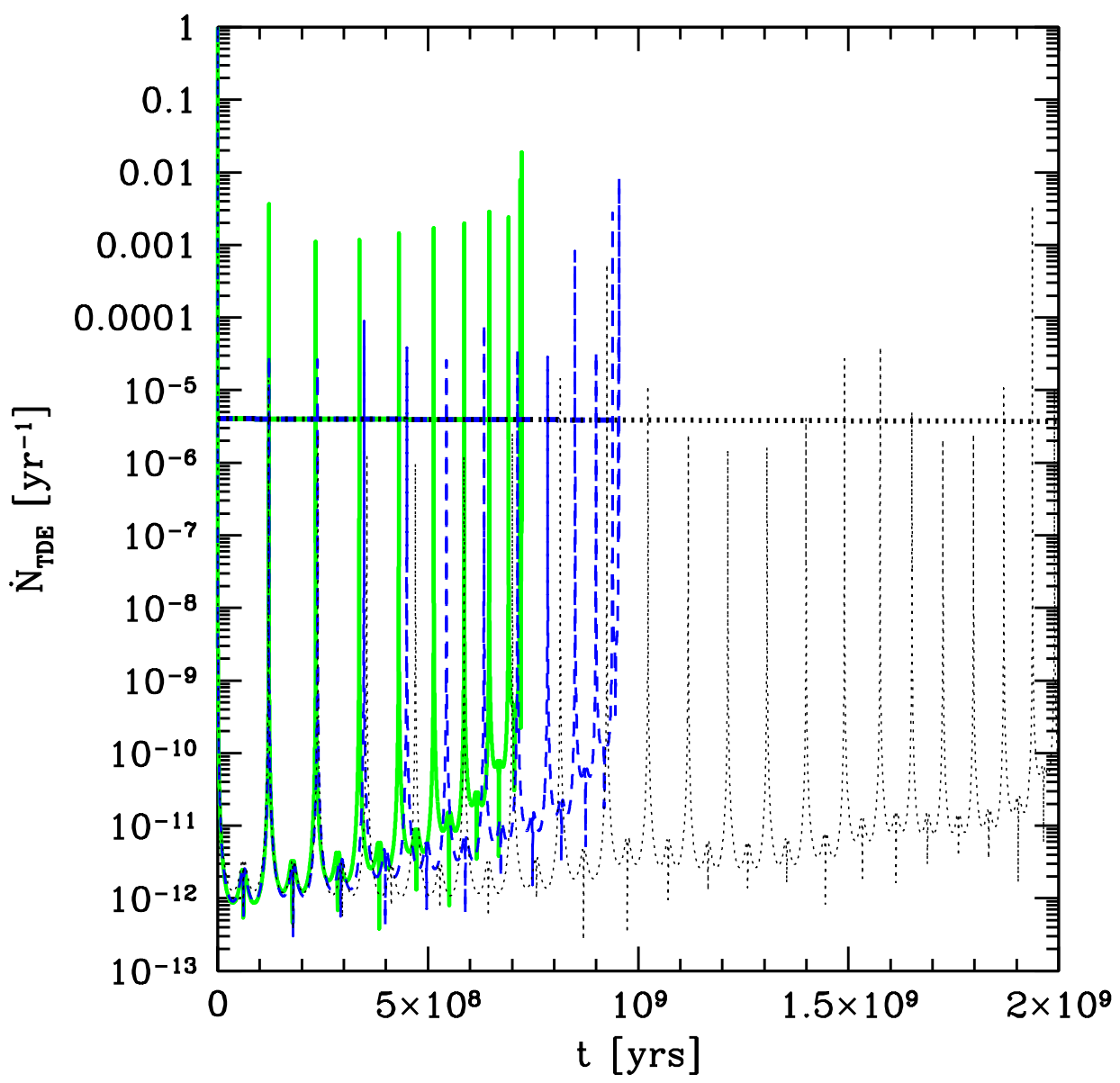

Figure 3.3.-: The number of stars, $\dot{N}_{\mathrm{TDE}}$, disrupted per year for the SMBHs in previous figures. The thick lines refer to disruptions from the bound cloud, while the thin lines refer to disruptions of unbound stars. Colors and line types represent the same black holes as in Figure 3.1. 


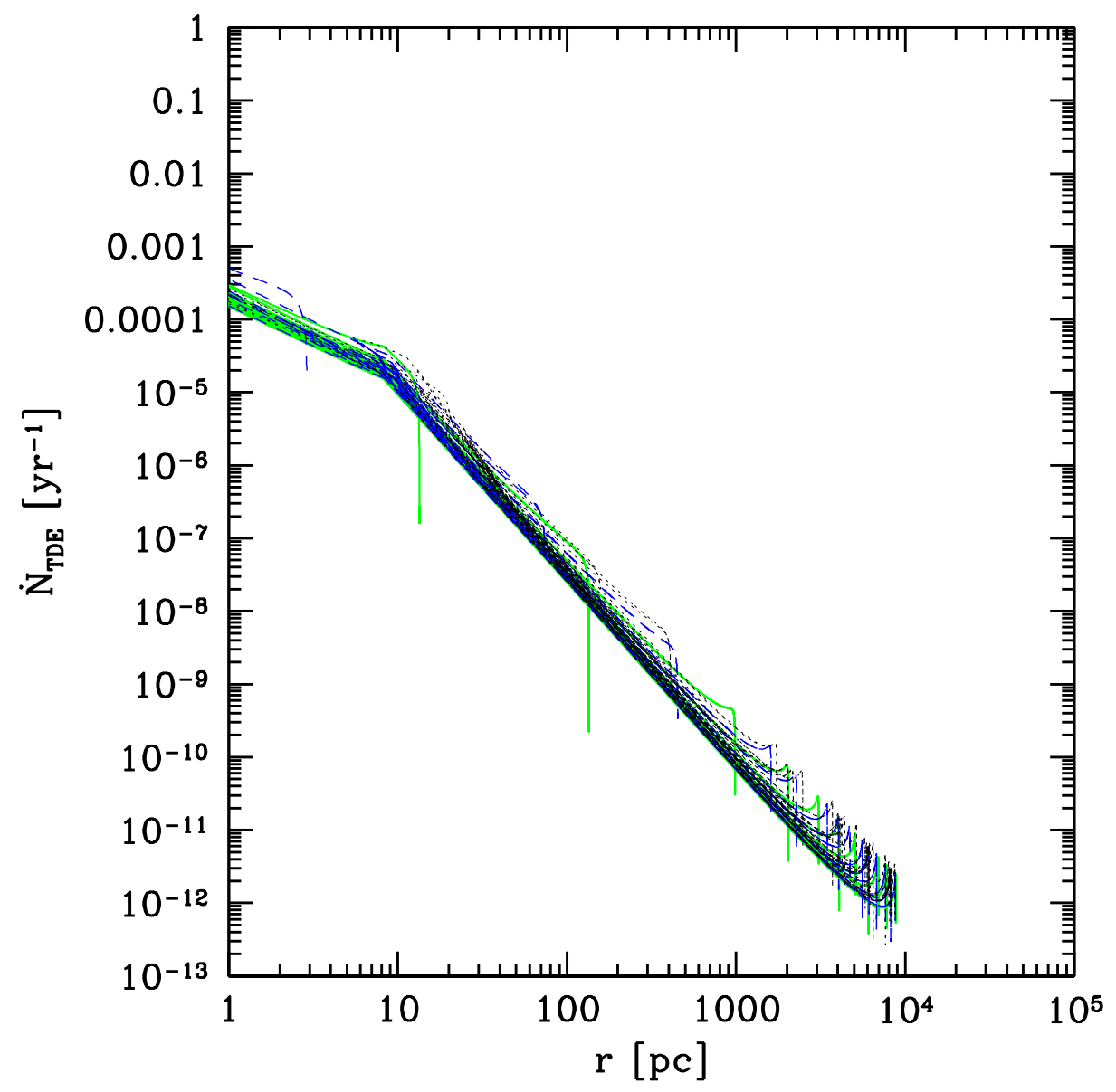

Figure 3.4.-: The rate of unbound stellar disruption, $\dot{N}_{\mathrm{TDE}}$, as a function of radius for the SMBHs in previous figures. 


\section{CHAPTER 3. OFFSET TDES FROM RECOILED SMBHS}

interesting values of $\Upsilon$. Both bound and unbound disruption rates are dominated by the highest black hole mass permitted by its spin amplitude to disrupt stars; for the first row (fiducial $a$ values), this corresponds to $10^{7.5} M_{\odot}$, while for the second row $(a=0.99)$ it is $10^{8} M_{\odot}$ and for the third row $(a=0.0)$ it is $10^{7} M_{\odot}$. We note here that $10^{7} M_{\odot}$ black holes can always disrupt solar-type stars, but require a modest amount of spin in order for the ISCO to lie inside the tidal radius, which we take as a precondition for either disk or super-Eddington outflow emission (thereby neglecting the short-lived X-ray shock breakout signature of the TDE, explored in Guillochon et al. (2009), which LSST would not detect). Almost all bound cloud disruptions have an observable spatial offset, while unbound disruptions never have an observable offset. On the other hand, a higher fraction of unbound disruptions possess an observable kinematic offset relative to the bound cloud disruptions. Both these correlations are easily explainable: due to high orbital eccentricity, the SMBHs in our sample spend the majority of their time far from the galactic nucleus, so most bound cloud disruptions occur with a large physical offset and low velocity. At the same time, virtually all (see

Figure 3.3) unbound disruptions occur during perigalacticon, where the SMBHs move at their highest velocities. The highly aligned $\left(<10^{\circ}\right)$ progenitor spin model produces a negligible number of disruptions; high kick velocities are suppressed, and SMBHs escape the galactic nucleus too infrequently to disrupt significant numbers of stars.

In Figure 3.6, we display $\mathrm{d} \Upsilon / \mathrm{d} \ln M_{\mathrm{BH}}$ for models with super-Eddington flares. The results are similar to those in Figure 3.5, although $\mathrm{d} \Upsilon / \mathrm{d} \ln M_{\mathrm{BH}}$ is everywhere greater than or equal to its values in the previous figure. Two special points of contrast are the large increase in observable disruptions at the low end of the SMBH mass function, and the (corresponding) increase in disruptions for the highly aligned progenitor spin 


\section{CHAPTER 3. OFFSET TDES FROM RECOILED SMBHS}

model. The addition of super-Eddington flares has, as expected, little effect on values of $\mathrm{d} \Upsilon / \mathrm{d} \ln M_{\mathrm{BH}}$ above $10^{7} M_{\odot}$, but disruption flares become dramatically more visible for $10^{6} M_{\odot}$ and $10^{6.5} M_{\odot}$ SMBHs.

Figure 3.7 displays $\mathrm{d} \Upsilon_{\mathrm{s}} / \mathrm{d} \ln M_{\mathrm{BH}}$, the mass dependence of the total observable (spatially offset) rate $\Upsilon_{\mathrm{s}}$, integrated over all kick velocities and all galaxies in our mock catalog, and given fiducial spin values. $\Upsilon_{\mathrm{s}}$ is, as discussed in $\S 2.6$, the rate of spatially offset flares that will be identified as offset by LSST's automatic photometric subtraction, without any followup observations. When we integrate over black hole mass, we find that two of our kick velocity distributions produce a robustly observable $(\sim 10)$ number of disruptions per year assuming super-Eddington flares, while the third produces a more marginal number of TDEs, of order unity per year. Likewise, progenitor spin distributions aligned to within $180^{\circ}$ or within $30^{\circ}$ produce $\sim 1$ flare per year with an observable spatial offset if we are only able to observe disk emission. Higher-mass SMBHs contribute the most to observable disk flares, due to the lower temperatures and higher optical luminosities of their disks, while super-Eddington accretion flares are dominated by the lower-mass end of the SMBH distribution. Although the rate enhancement from inclusion of super-Eddington outflows is almost a factor of 10, this is considerably lower than the comparable factor in SQ09. The reason for this disparity is that the brightest super-Eddington outflows correspond to the deepest plunges (lowest $r_{\mathrm{p}}$ ) into the tidal disruption region. SQ09 considered a logarithmically flat distribution of $r_{\mathrm{p}}$, while we took a constant $r_{\mathrm{p}}=r_{\mathrm{t}}$, for the reasons explained in $\S 3$.

A variety of observable TDE rates are displayed in Table 1 . These numbers have been integrated over galaxy type, kick velocity distribution, inclination angle, and black hole mass function, and indicate that the ultimate observability of recoil-induced TDEs 


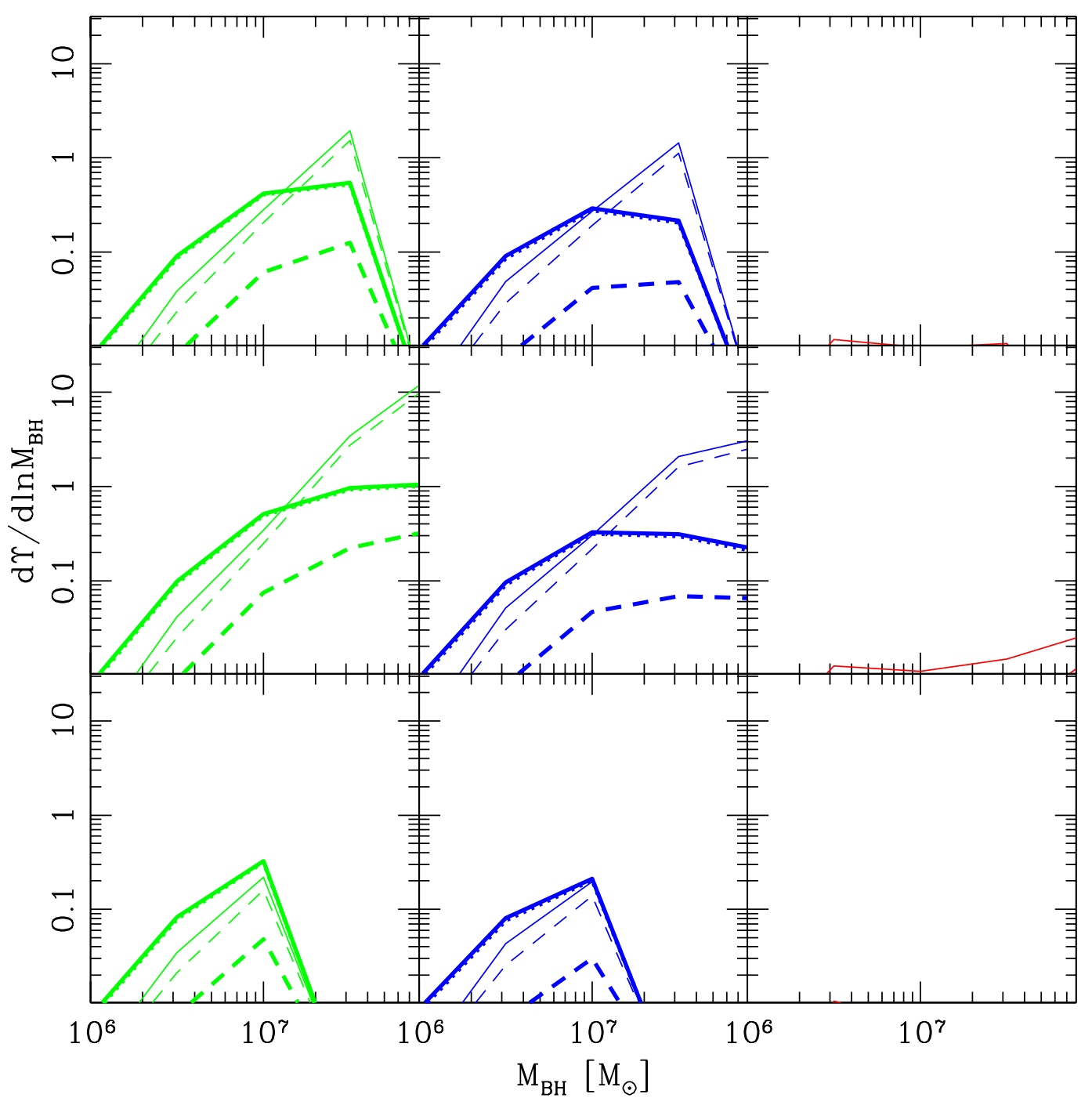

Figure 3.5.-: Mass dependence of total TDE rates, $\Upsilon$. In the left (green) column, we show the unaligned spin case; in the middle (blue), spins aligned to within $30^{\circ}$; and the right (red), spins aligned to within $10^{\circ}$. The top row has fiducial final spin amplitudes, while the middle has $a=0.99$ and the bottom row has $a=0.0$. The thick lines represent disruptions of bound stars; the thin lines, disruptions of unbound stars. The solid lines represent total number of disruptions, while dotted lines represent disruptions with observable spatial offsets and dashed lines represent disruptions with observable kinematic offsets. In this plot only disk emission is considered. 


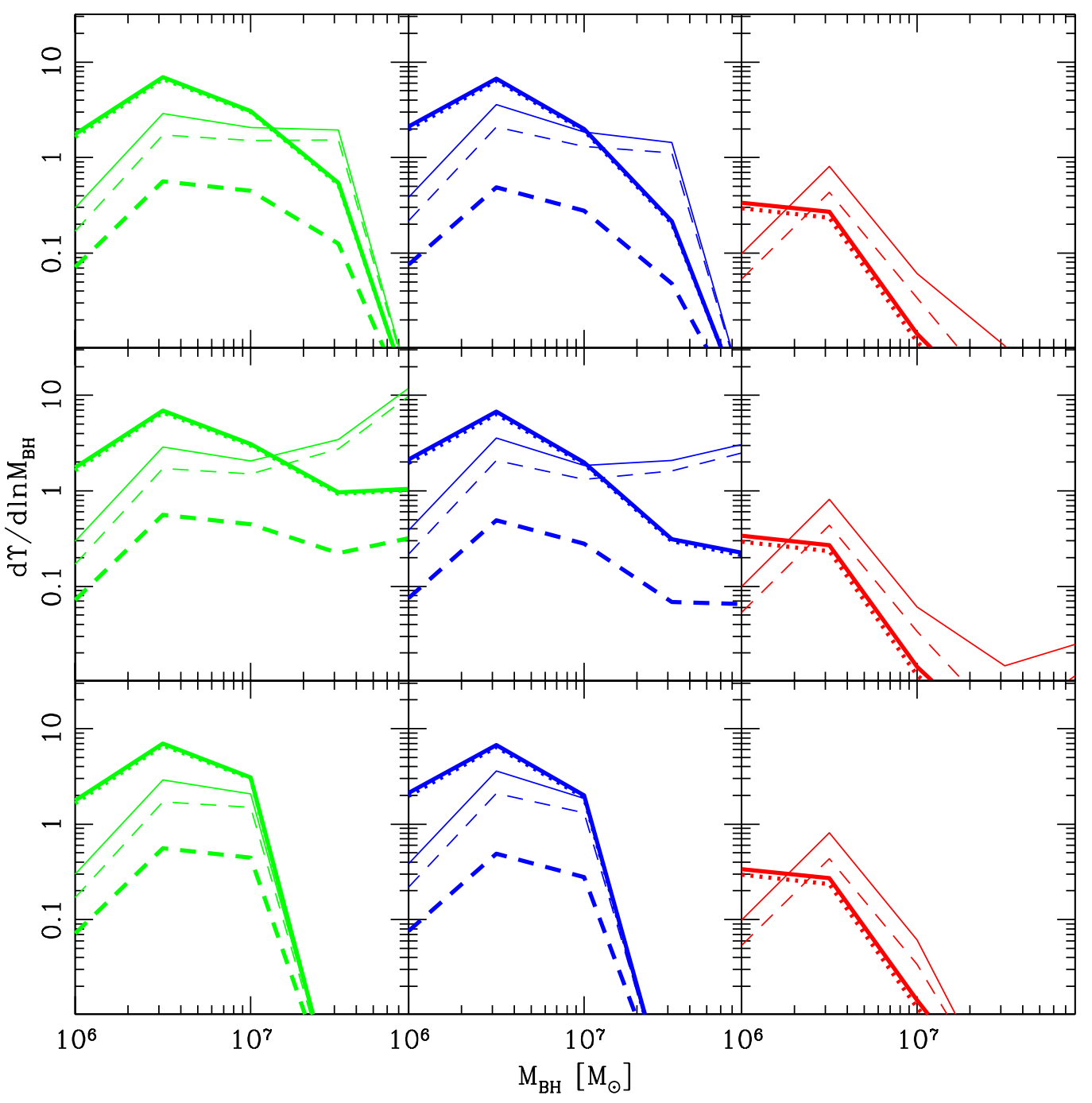

Figure 3.6.- : The same as the previous figure, but assuming the existence of superEddington outflows. 


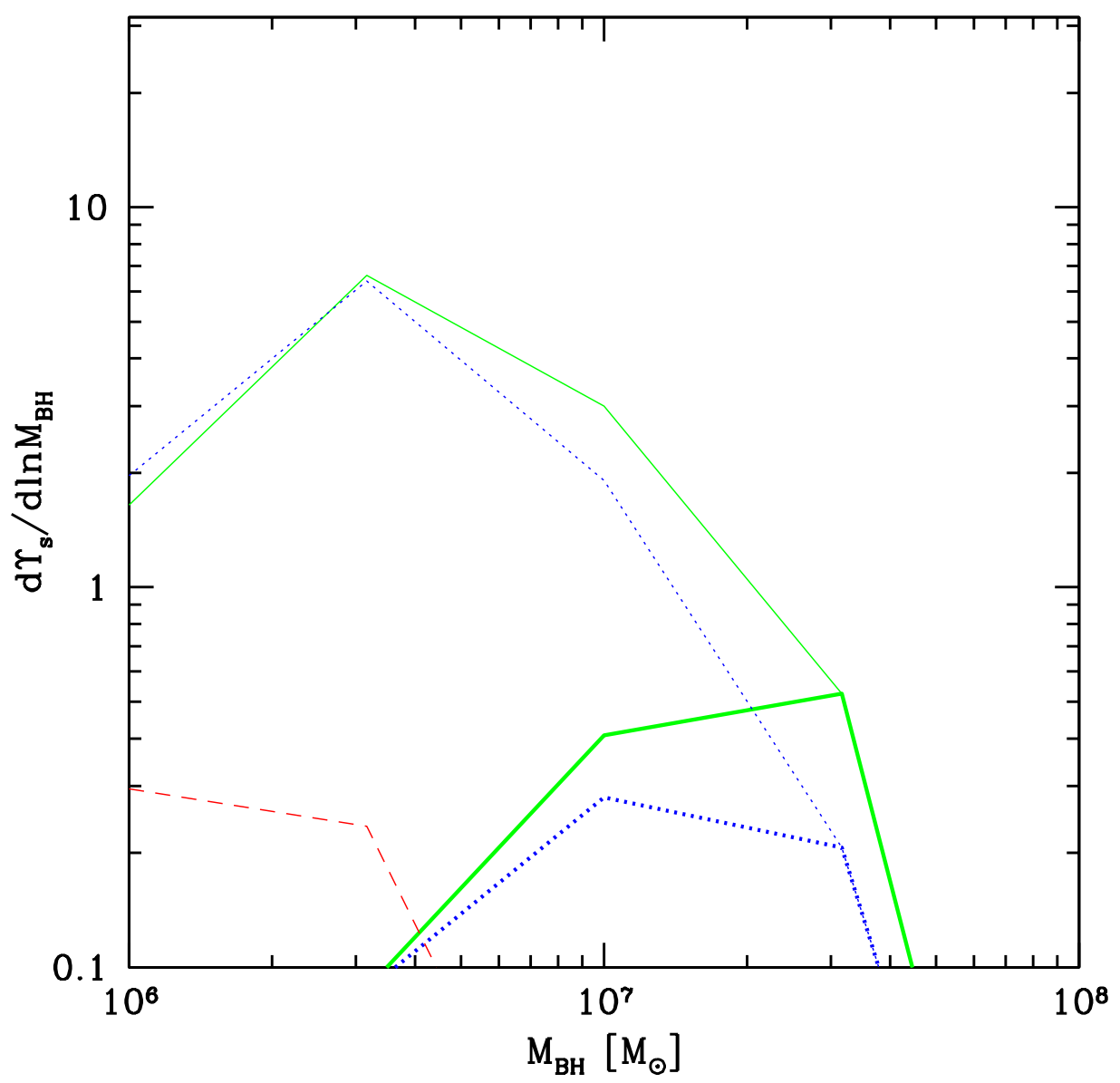

Figure 3.7.- : Mass dependence of the total, galaxy- and velocity-averaged rate of spatially offset TDEs, $\Upsilon_{\mathrm{s}}$, in the case of fiducial spin. $180^{\circ}, 30^{\circ}$, and $10^{\circ}$ progenitor spin alignment correspond to solid green lines, dotted blue lines, and dashed red lines, respectively. Thick lines represent disk emission only, while the thin lines correspond to disk plus super-Eddington outflows. 


\section{CHAPTER 3. OFFSET TDES FROM RECOILED SMBHS}

will depend strongly on both the existence of super-Eddington flares, and the average distribution of pre-merger spin alignments. In this table, $\Upsilon$ values for fiducial SMBH spins are shown, with the $a=0$ and $a=0.99$ cases appearing as lower and upper limits in parentheses. It is only in the case where super-Eddington flares do not exist and substantial progenitor spin alignment occurs where we expect LSST to observe negligible numbers of spatially offset TDEs per year. We note that if the progenitor spins are unaligned, or even aligned with scatter $\geq 30^{\circ}$, the tidal disruption rate from recoiling black holes is almost $1 \%$ of the total inferred TDE rate $\left(\sim 10^{-5} \mathrm{yr}^{-1}\right.$ per galaxy $)$ for all galaxies. For most of our models, the number of kinematically offset TDEs, $\Upsilon_{k}$, is comparable to $\Upsilon_{s}$, although we note again that the theoretical basis for expecting appropriate absorption lines in a super-Eddington outflow is less secure than that for a simple spatial offset. We have also included in Table 1 the rates of spatially and kinematically offset TDEs for SMBHs which escape their host galaxy altogether, labeling these as $\Upsilon_{\mathrm{s} \text {,esc }}$ and $\Upsilon_{\mathrm{k} \text {,esc }}$. Only in the case of unaligned spins and super-Eddington outflows are $\Upsilon_{\mathrm{s}, \mathrm{esc}} \sim \Upsilon_{\mathrm{s}}$ and $\Upsilon_{\mathrm{k}, \mathrm{esc}} \sim \Upsilon_{\mathrm{k}}$; in all other scenarios the number of observable TDEs due to ejected SMBHs is at least a factor of 7 smaller than the number due to bound SMBHs.

For most of the models we have considered, a large majority of the TDEs associated with recoiling SMBHs occur for black holes bound to their host galaxy. This is due to two factors: both the relatively low fraction of SMBHs recoiled at escape velocity (see Figure 3.8 for a plot of the SMBH escape fraction, $f_{\text {esc }}$ ), and the smaller, more rapidly decaying bound clouds of those low-mass SMBHs which do escape. This highlights the importance of searching for SMBHs bound to the bulge or halo of their host galaxy; although the intergalactic TDEs of the KM08 scenario offer a cleaner signal, they are 
CHAPTER 3. OFFSET TDES FROM RECOILED SMBHS

intrinsically much fewer in number. Our work has differed from KM08 primarily in considering much wider ranges of $v_{\mathrm{k}}$, using kick velocity distributions motivated by merger gas content. Our treatment of disruption rates for a given $v_{\mathrm{k}}$ is mostly similar, with the exception of incorporating the results of O'Leary \& Loeb (2012) for late-time relaxation in the bound cloud.

Finally, it is worth summarizing the primary assumptions we made in this work, where we have tried to err on the side of conservatism. To simplify our calculations, we neglected emission from the unbound TDE debris, although that can substantially increase optical (non-super-Eddington) emission for low-mass SMBHs (SQ09). Our SMBH wandering lifetimes were likely reduced by the fact that we limited ourselves to axisymmetric stellar bulge geometries, and even more importantly only considered spherical dark matter haloes. Our simple choice of stellar mass function is slightly conservative for calculations of TDE rate. 


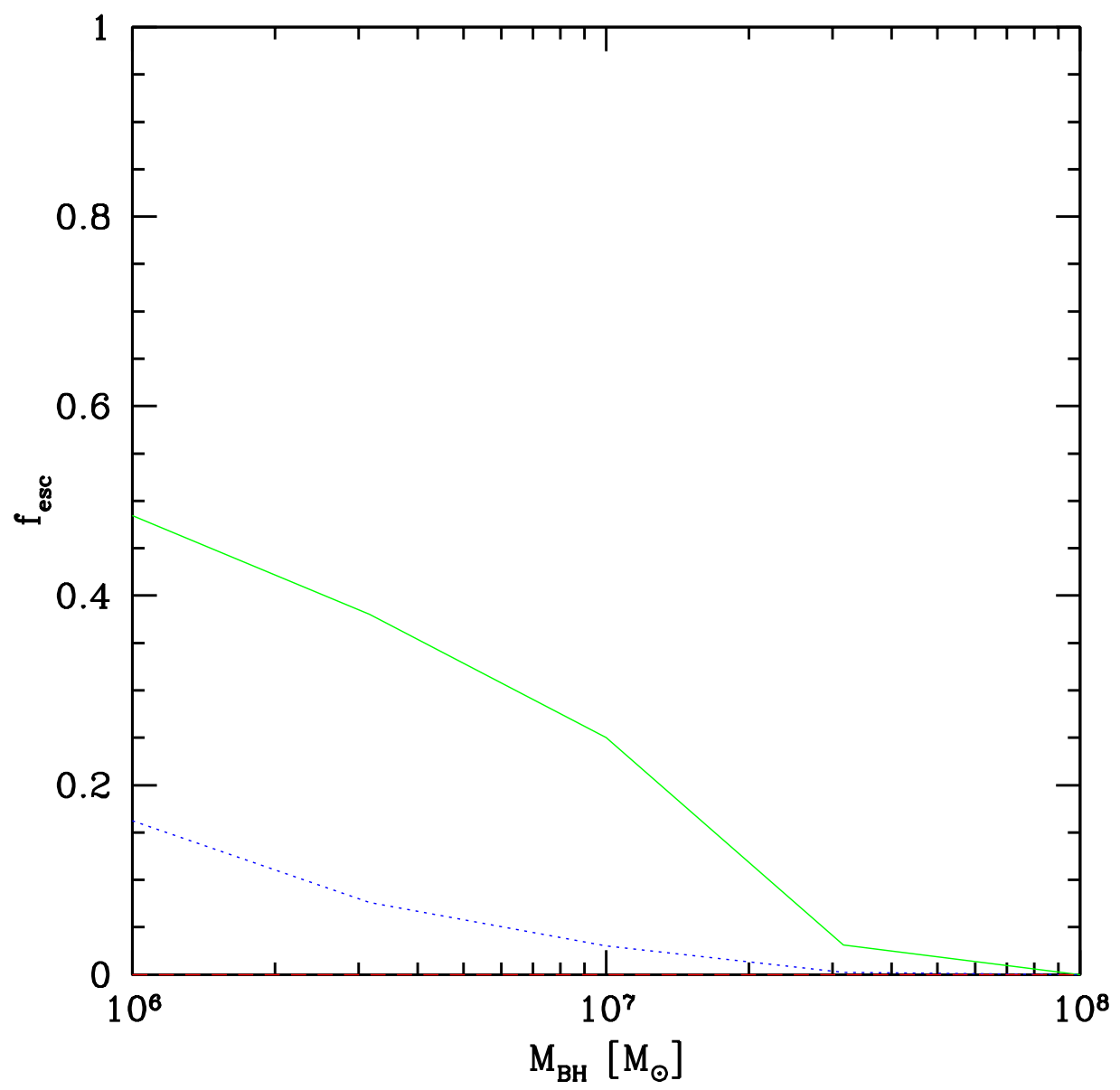

Figure 3.8.- : Fraction of recoiled SMBHs which escape into intergalactic space, as a function of black hole mass, for the three different kick velocity distributions. As in the previous figure, the green solid line represents the 180 degree alignment distribution, the dotted blue the 30 degree, and the dashed red (not visible; a negligible fraction of SMBHs from this distribution escaped their host galaxies) the 10 degree. 
CHAPTER 3. OFFSET TDES FROM RECOILED SMBHS

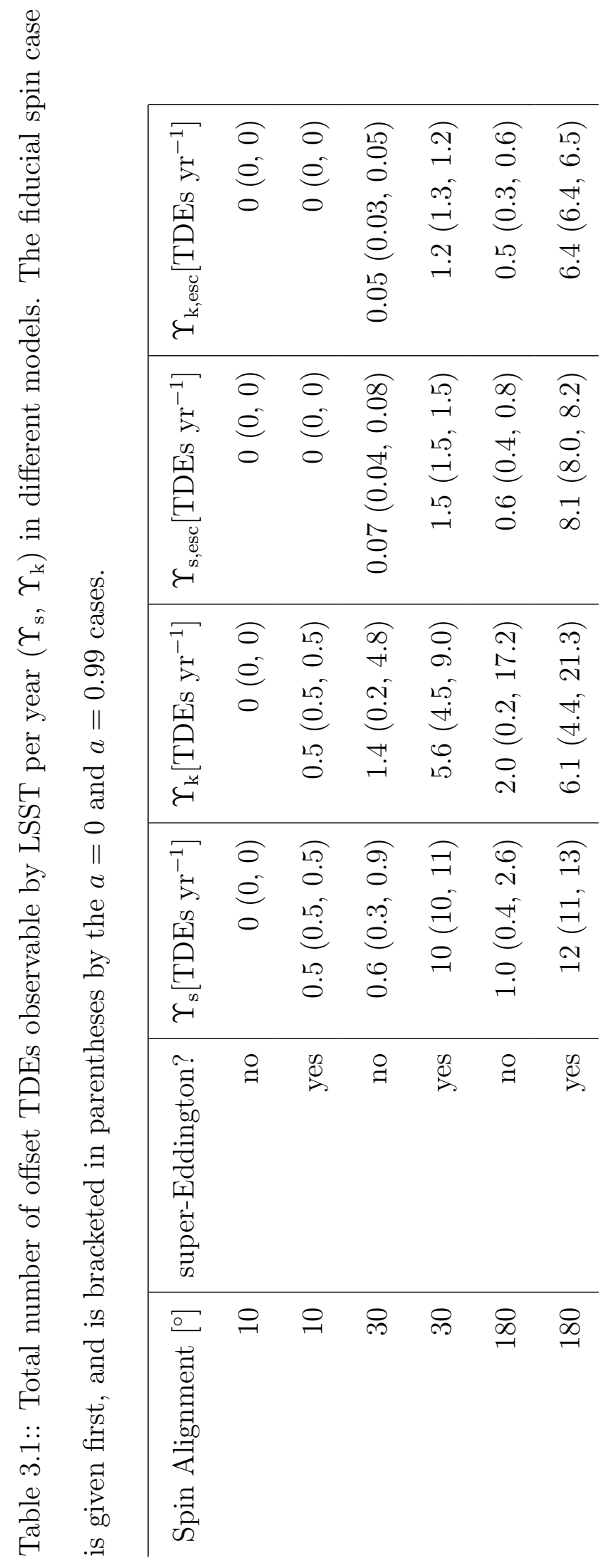




\subsection{Conclusions}

In this paper, we have generalized the work of KM08 to include a mock galaxy catalogue and physically motivated distributions of kick velocities, so as to estimate the observability of offset tidal disruption flares. We have demonstrated that superEddington flares from recoiling black holes, if they exist along the lines envisioned in SQ09, will be observably offset to LSST in numbers ranging from $\sim 1$ to $\sim 10$ TDEs

per year. This is true for a broad range of assumptions about kick velocity distributions and galactic structure. This subset of transients would contain important scientific value as evidence of black hole recoil, and could potentially constrain the $v_{\mathrm{k}}$ distribution. If super-Eddington flares do not exist or if they differ significantly from the SQ09 picture (for example, if $f_{\text {out }} \ll 0.1$ ), optical emission from the accretion disks of TDEs around recoiling black holes will still be accessible to LSST, although here the case is more marginal. If a large fraction of local universe SMBH mergers proceed without significant spin alignment, the prospects for optical detection of disk emission from recoiling TDEs are relatively good, but moderate amounts of alignment would likely suppress this. Importantly, the majority of recoiled SMBHs will remain bound to their host galaxies, making photometric subtraction critical for identification of recoil-associated disruption flares. Depending on the nature of the super-Eddington phase of accretion, a comparably large population of kinematically offset flares is potentially detectable, but would require spectroscopic followup to be realized.

We have also shown that confusion with TDEs from stationary SMBHs will not be a major challenge in the detection of off-nuclear TDEs, leaving supernova contamination as the main concern. If the scientific potential of spatially offset TDEs is to be utilized, it 
will be necessary to construct transient survey pipelines which do not employ the typical "galactic center" cut when searching for TDEs. Although the challenges inherent to TDE identification have been discussed elsewhere (van Velzen et al. 2011b), the distinctive lightcurve and color evolution of tidal disruption flares are helpful in separating them. The large number of TDEs expected to be observed by time domain surveys in the coming decade will calibrate our understanding of these events, so that once LSST is online, it may be able to confirm the SMBH recoil predictions of numerical relativity. 


\section{Chapter 4}

\section{Consequences of Strong Compression in Tidal Disruption Events}

N. Stone, R. Sari, \& A. Loeb accepted in The Monthly Notices of the Royal Astronomical Society, 2013

\section{Abstract}

The tidal disruption of a star by a supermassive black hole (SMBH) is a highly energetic event with consequences dependent on the degree to which the star plunges inside the SMBH's tidal sphere. We introduce a new analytic model for tidal disruption events (TDEs) to analyze the dependence of these events on $\beta$, the ratio of the tidal radius to the orbital pericenter. We find, contrary to most previous work, that the spread in debris energy for a TDE is largely constant for all $\beta$. This result has important consequences for optical transient searches targeting TDEs, which we discuss. We 


\section{CHAPTER 4. STRONG COMPRESSION IN TDES}

quantify leading-order general relativistic corrections to this spread in energy and find that they are small. We also examine the role of stellar spin, and find that a combination of spin-orbit misalignment, rapid rotation, and high $\beta$ may increase the spread in debris energy. Finally, we quantify for the first time the gravitational wave emission due to the strong compression of a star in a high- $\beta$ TDE. Although this signal is unlikely to be detectable for disruptions of main sequence stars, the tidal disruption of a white dwarf by an intermediate mass black hole can produce a strong signal visible to Advanced LIGO at tens of megaparsecs.

\subsection{Introduction}

Stars which pass too close to supermassive black holes (SMBHs) are disrupted by the enormous gravitational gradients acting on them. The eventual fallback of $\sim 50 \%$ of the star's mass onto the black hole can produce a highly luminous, multiwavelength flare the primary observable signature of a tidal disruption event (TDE). Over a dozen strong TDE candidates have been observed, with most detections made in X-ray (Bade et al. 1996; Komossa \& Greiner 1999; Gezari et al. 2003) or UV (Gezari et al. 2006, 2008, 2009), but some in optical archival (van Velzen et al. 2011b) and transient (Cenko et al. 2012a; Gezari et al. 2012) searches. Recently, collimated jets from two relativistic TDE candidates have been detected by the Swift satellite (Levan et al. 2011; Bloom et al. 2011; Zauderer et al. 2011; Cenko et al. 2012b).

The rate of TDEs is highly uncertain, from both theoretical and observational perspectives. On the observational side, uncertainties stem from both the low sample size and unclear sources of systematic error; nonetheless, observational estimates of the TDE 


\section{CHAPTER 4. STRONG COMPRESSION IN TDES}

rate per galaxy generally find $\dot{N}_{\mathrm{TDE}} \sim 10^{-4}-10^{-5} \mathrm{yr}^{-1}$ (Donley et al. 2002; Gezari et al. 2008). This is in rough agreement with the wide range of theoretical predictions for the TDE rate, which invoke different ways to scatter stars into the SMBH "loss cone" (the region of low angular momentum phase space containing orbits which pass inside the tidal sphere). The most theoretically secure method of feeding stars into the loss cone is standard two-body relaxation, which sets a conservative lower limit on $\dot{N}_{\text {TDE }}$ between $10^{-4}$ and $10^{-6} \mathrm{yr}^{-1}$ (Frank \& Rees 1976; Lightman \& Shapiro 1977; Cohn \& Kulsrud 1978; Magorrian \& Tremaine 1999; Wang \& Merritt 2004). Alternative mechanisms for enhancing the TDE rate include triaxiality in a galaxy's nuclear potential (Merritt \& Poon 2004), encounters with massive perturbers (Perets et al. 2007), the effect of an inspiraling secondary SMBH (Ivanov et al. 2005; Chen et al. 2009, 2011; Wegg \& Nate Bode 2011), and gravitational wave recoil after the merger of a binary SMBH (Stone \& Loeb 2011).

Past theoretical work on TDEs has included analytic estimates of event energetics and timescales (Rees 1988; Phinney 1989; Ulmer 1999; Strubbe \& Quataert 2009), hydrodynamical simulations of the disruption process in both smoothed-particlehydrodynamics (Nolthenius \& Katz 1982; Evans \& Kochanek 1989; Laguna et al. 1993; Lodato et al. 2009) and mesh (Khokhlov et al. 1993a; Frolov et al. 1994; Guillochon et al. 2009; Guillochon \& Ramirez-Ruiz 2013) codes, and radiative transfer work to quantify emission and absorption processes in TDEs (Kasen \& Ramirez-Ruiz 2010; Strubbe \& Quataert 2011). The large hierarchy of time and length scales involved in a TDE makes it difficult to self-consistently simulate one from disruption to the onset of accretion, so other work has focused on the formation (Kochanek 1994) and evolution (Cannizzo et al. 1990; Montesinos Armijo \& de Freitas Pacheco 2011) of TDE accretion 


\section{CHAPTER 4. STRONG COMPRESSION IN TDES}

disks. The properties of these flares depend crucially on the spread in orbital specific energy of the post-disruption debris streams, as that spread sets the mass fallback rate onto the SMBH. All fallback rates are generally expected to produce multicolor blackbody emission from the accretion disks they feed (Lodato \& Rossi 2011), while super-Eddington fallback rates may drive powerful outflows, increasing the optical luminosity by orders of magnitude (Loeb \& Ulmer 1997; Strubbe \& Quataert 2009, 2011). Weaker, nondisruptive tidal encounters can be studied as linear perturbations

to an equilibrium stellar structure (Press \& Teukolsky 1977; Gomboc \& Čadež 2005), although as orbital pericenters approach the tidal radius hydrodynamical simulations are necessary to capture nonlinear effects and partial mass loss (Khokhlov et al. 1993b; Diener et al. 1997; Guillochon \& Ramirez-Ruiz 2013).

Much of the pioneering work on TDEs was done in the 1980s using the semi-analytic affine model (Carter \& Luminet 1983, hereafter CL83), which treats the disrupting star as a set of concentric ellipsoidal shells evolving under the combined influences of self-gravity, pressure, and the SMBH tidal field (Carter \& Luminet 1985; Luminet \& Carter 1986). This model has found a wide range of uses, and has been generalized to include both thermonuclear reaction networks (Luminet \& Pichon 1989) and general relativistic (GR) effects (Luminet \& Marck 1985), although its validity tends to break down at late times as the stellar debris exits the tidal sphere. One key finding of the affine model is that during the early stages of disruption, prior to the star's arrival at pericenter, motion orthogonal to the orbital plane decouples from motion within, leading to a strong, one-dimensional compression (a vertical "pancaking") of the star. This effect scales strongly with the penetration factor $\beta$, defined as the ratio of the tidal radius $R_{\mathrm{t}}$ to the pericenter radius $R_{\mathrm{p}}$. The pancaking of the star is reversed by 


\section{CHAPTER 4. STRONG COMPRESSION IN TDES}

the buildup of internal pressure, which leads to a rebound in the vertical direction. Shock formation accompanies this rebound (and occasionally the infall period prior to maximum compression), with X-ray shock breakout a potential though as yet undetected observational signature of TDEs (Kobayashi et al. 2004; Guillochon et al. 2009).

In this paper, after establishing basic dynamical features of TDEs ( $(2)$ we present a new analytic model to analyze the tidal free fall of the star prior to its maximum vertical compression (§3). In many ways, this represents a simplification of the affine model, and its primary appeal is its greater analytic tractability. Using our model we correct a longstanding error in the literature on the spread in debris energy $\Delta \epsilon$. We verify the robustness of our estimates by considering redistribution of vertical collapse energy to in-plane motion $(\S 4)$, the desynchronization of vertical collapse $(\S 5)$, and leading-order GR corrections (§6), the latter of which are found to be small. We examine the gravitational waves (GWs) generated by rapid changes in the star's quadrupole moment during maximum compression, and find them to be detectable by Advanced LIGO for disruptions of white dwarfs $(\S 7)$. We conclude with the observational implications of our work, which primarily involve the suppression of strongly super-Eddington TDEs (§8), and a general discussion $(\S 9)$.

\subsection{Dynamical Energy Spread}

A star is tidally disrupted if the pericenter of its orbit, $R_{\mathrm{p}}$, lies inward of the tidal radius,

$$
R_{\mathrm{t}}=R_{*}\left(M_{\mathrm{BH}} / M_{*}\right)^{1 / 3} .
$$




\section{CHAPTER 4. STRONG COMPRESSION IN TDES}

Here $M_{*}$ and $R_{*}$ are the mass and radius of the victim star, and $M_{\mathrm{BH}}$ is the black hole mass. In reality, this expression for the tidal radius is not exact and contains weak, order unity dependences on stellar structure (Diener et al. 1995), stellar spin, and black hole spin (Kesden 2012b). We ignore these complications in this paper. Very shortly after entry into the tidal sphere (and before pericenter passage for $\beta>1$ ), the SMBH's tidal forces do an amount of work exceeding the star's gravitational binding energy, and the star's fluid elements begin moving on roughly geodesic trajectories. In the standard picture, hydrodynamic forces are subsequently neglected and the specific orbital energy $\epsilon$ of the debris streams is "frozen in," with a spread given by

$$
\Delta \epsilon=k \frac{G M_{\mathrm{BH}} R_{*}}{R_{\mathrm{p}}^{2}},
$$

where $G$ is the gravitational constant and $k$ a constant of order unity related to stellar structure and rotation prior to disruption. This approximate estimate can be obtained by Taylor-expanding the SMBH potential around the star at pericenter, or alternatively by multiplying the equivalent tidal acceleration at pericenter $A_{\mathrm{p}} \sim\left(G M_{\mathrm{BH}} / R_{\mathrm{p}}^{2}\right)\left(R_{*} / R_{\mathrm{p}}\right)$ by the dynamical time $T_{\mathrm{p}} \sim\left(G M_{\mathrm{BH}} / R_{\mathrm{p}}^{3}\right)^{-1 / 2}$ to get $\Delta V_{\mathrm{p}}=A_{\mathrm{p}} T_{\mathrm{p}}$. Using $V_{\mathrm{p}}=$ $\left(2 G M_{\mathrm{BH}} / R_{\mathrm{p}}\right)^{1 / 2}$, one can then find $\Delta \epsilon=V_{\mathrm{p}} \Delta V_{\mathrm{p}} \sim G M_{\mathrm{BH}} R_{*} / R_{\mathrm{p}}^{2}$. We note that Eq. 4.2 is widely used in the literature (Evans \& Kochanek 1989; Kochanek 1994; Ulmer 1999; Kasen \& Ramirez-Ruiz 2010; Strubbe \& Quataert 2009; Lodato \& Rossi 2011).

However, this reasoning is incorrect; by the time the star reaches pericenter its fluid elements are moving on almost ballistic trajectories. As the star plunges into the tidal sphere, internal forces become subdominant to the SMBH tidal field, with the ratio of the tidal to the self-gravitational acceleration given by $a_{\mathrm{t}} / a_{\mathrm{g}} \approx\left(R_{\mathrm{t}} / R\right)^{3}$. Here $R$ is the orbital separation. The work done by internal forces decreases more slowly, 


\section{CHAPTER 4. STRONG COMPRESSION IN TDES}

$\sim G M_{*} R / R_{*}^{2}$, although this simple expression overestimates the amount of work done by internal forces, which at $R \approx R_{\mathrm{t}}$ will self-cancel each other to first order (given that the star is initially in hydrostatic equilibrium).

To accurately evaluate $\Delta \epsilon$ at pericenter passage, one would need to account for distortions in the free-falling star's physical shape, as well as internal velocities. At any point along the star's orbit, a Cartesian coordinate system (Brassart \& Luminet 2008) will define the principal axes (eigenvectors) of the tidal tensor. If we define $\hat{X}$ parallel to the vector connecting the star and SMBH, $\hat{Y}$ in the orbital plane but perpendicular to $\hat{X}$, and $\hat{Z}$ perpendicular to the orbital plane, the star will be stretched in the $\hat{X}$ direction but compressed in the $\hat{Y}$ and $\hat{Z}$ directions. By the time the star reaches pericenter, the rotation of these axes along a parabolic orbit has resulted in compression along the $\hat{X}$ axis which reduces the potential gradient across the star, invalidating the above formula; further inaccuracy is introduced by the internal motions (i.e. velocity shear among ballistic debris trajectories) of the star within the SMBH's tidal sphere. A more accurate estimate of the spread in specific energy can be found by taking the potential gradient at the moment of tidal disruption, i.e. when the star crosses the tidal sphere and becomes unbound, as after this point the motion of the debris becomes roughly geodesic. This revision to the approximation of energy freeze-in yields

$$
\Delta \epsilon=k \frac{G M_{\mathrm{BH}} R_{*}}{R_{\mathrm{t}}^{2}} .
$$

We note that an analogous conclusion (on the $\beta$-independence of the energetics of tidal disruption) can be seen in tidal separations of binary stars by SMBHs (Sari et al. 2010, hereafter SKR10). We can alternatively use $\beta=R_{\mathrm{t}} / R_{\mathrm{p}}$ to rewrite Eqs. $4.2,4.3$ as

$$
\Delta \epsilon=k \beta^{n} \frac{G M_{\mathrm{BH}} R_{*}}{R_{\mathrm{t}}^{2}},
$$


CHAPTER 4. STRONG COMPRESSION IN TDES

with $n=2$ for the standard, Eq. 4.2 picture and $n=0$ for our revised, Eq. 4.3 analysis. In the sections below, our more detailed analysis of the tidal compression experienced by the star will examine if intermediate or piecewise values of $n$ are more appropriate. The observational implications of changes to $\Delta \epsilon$ are discussed in $\S 8$.

\subsection{Free Solutions, and Free Collapse}

Other factors could influence or invalidate the simple analytic argument presented in $\S 2$, such as redistribution of energy during the moments of maximum compression, GR corrections, stellar spin, or simply work done on the star's fluid elements by subdominant internal forces inside the tidal sphere. In this section, we introduce a new analytic model for the tidal free fall of a disrupted star that will help us approach these issues.

Because the dominant source of TDEs is expected to be stars scattered onto radial orbits from $\sim$ pc scales (Magorrian \& Tremaine 1999; Wang \& Merritt 2004), we assume a parabolic orbit for the center of mass of the star, with distance from the SMBH given by:

$$
R=\frac{2 R_{\mathrm{p}}}{1+\cos f} .
$$

For such an orbit time $t$ is related to true anomaly $f$ via

$$
t=\frac{1}{3}\left(\frac{2 R_{\mathrm{p}}^{3}}{G M_{\mathrm{BH}}}\right)^{1 / 2} \tan (f / 2)\left(3+\tan ^{2}(f / 2)\right),
$$

although the differential form

$$
\frac{d f}{d t}=\frac{1}{8^{1 / 2}}(1+\cos f)^{2} \sqrt{\frac{G M_{\mathrm{BH}}}{R_{\mathrm{p}}^{3}}}
$$

is more generally useful. We set $t=f=0$ at $R=R_{\mathrm{p}}$, and use $\dot{f}>0$ throughout this 


\section{CHAPTER 4. STRONG COMPRESSION IN TDES}

paper.

The sequence of events in a TDE, first noted by CL83, will be useful shorthand for us, so we introduce it here. Phase I (near-equilibrium) of a TDE lasts while $R>R_{\mathrm{t}}$, and the star remains in approximate if slightly perturbed equilibrium. Phase II (free fall) begins when the star crosses the tidal sphere and becomes gravitationally unbound; in this paper we will treat the transition between Phases I and II as instantaneous, an assumption we justify below in $§ 5$. The assumption of tidal free fall is very useful because of the existence of analytic, "free" solutions to the Hill equations in the parabolic restricted 3-body problem, but it is not immediately obvious that internal forces in the star can be neglected for $R<R_{\mathrm{t}}$. To first order the approximation seems reasonable because the ratio of tidal acceleration to self-gravitational acceleration grows quickly, as $a_{\mathrm{t}} / a_{\mathrm{g}} \approx\left(R_{\mathrm{t}} / R\right)^{3}$ for the bulk of the star. Furthermore, the star's internal pressure and self-gravity partially cancel each other, further reducing their combined contribution. For now, we assume the validity of the free fall assumption, but after developing further machinery we will justify it further in $§ 5$.

During this free fall, the star is compressed perpendicular to the orbital plane (along $\hat{Z}$ ) and in one direction within the orbital plane, while being stretched along the other in-plane direction. Although for the limiting case of radial infall the problem is self-similar in all three dimensions (SKR10), the rotation of the line connecting the star's center of mass to the SMBH breaks the in-plane similarity. By the time the star has reached pericenter, the $\hat{X}$ direction (which is parallel to the line between the SMBH and the orbital pericenter), is compressed, and $\hat{Y}$ is stretched, but the distortions are both much smaller than the compression orthogonal to the orbital plane. Shortly after passing

pericenter, synchronous tidal free fall in the $\hat{Z}$ direction leads to very strong compression 


\section{CHAPTER 4. STRONG COMPRESSION IN TDES}

of the star, which is eventually reversed by hydrodynamic forces. Phase III (bounce) begins when hydrodynamical forces become strong enough to begin slowing the collapse of the star along its vertical axis. Once the star's collapse has reversed, hydrodynamical forces quickly become negligible again, and Phase IV (the rebound) begins, with stellar gas once again moving on ballistic trajectories.

We take as initial conditions for Phase II a spherically symmetric star at the tidal sphere, with fluid elements possessing initial positions $\vec{r}$ (the coordinate origin tracks the star's center of mass) and initial velocities in the center of mass frame $\vec{u}(\vec{r})$. Making the approximation that upon entering the tidal sphere, internal forces become negligible unless and until compression triggers shock formation or isentropic pressure buildup, we take the pre-shock trajectories of these fluid elements to be completely ballistic. This means that their trajectories are given by the parabolic Hill equations, $\vec{r}_{\mathrm{H}}=\left\{x_{\mathrm{H}}, y_{\mathrm{H}}, z_{\mathrm{H}}\right\}$. Unlike the $\{\hat{X}, \hat{Y}, \hat{Z}\}$ coordinates, which rotate as $f$ progresses, the $\{\hat{x}, \hat{y}, \hat{z}\}$ which are used in the Hill equations define a fixed lab frame. The free solutions to these equations, neglecting self-gravity, can be written in closed form (SKR10) using coordinates where distance has been normalized by $R_{*}$ and time by $\sqrt{R_{*}^{3} /\left(G M_{*}\right)}$; we denote such coordinates in this paper by writing tildes over them. All other coordinates are in physical units, unless otherwise noted. The equations of motion themselves are derived in Appendix A. Although there are 6 independent solutions to these equations, motion out of the orbital plane is decoupled from motion within it, so only two are 
CHAPTER 4. STRONG COMPRESSION IN TDES

relevant for perturbed motion in the $\hat{z}$ direction:

$$
\begin{gathered}
\tilde{z}_{\mathrm{H}}=E \tilde{z}_{\mathrm{E}}+F \tilde{z}_{\mathrm{F}} \\
\tilde{z}_{\mathrm{E}}=\frac{1}{\beta} \frac{2 \sin f}{1+\cos f} \\
\tilde{z}_{\mathrm{F}}=\frac{1}{\beta} \frac{2 \cos f}{1+\cos f} .
\end{gathered}
$$

Here $E$ and $F$ are undetermined coefficients that are set by the initial conditions described above. In particular, if we require that a fluid element of initial position $z=z_{0}$ has initial velocity $w=\dot{z}=0$ at $f=f_{\mathrm{t}}$, where the true anomaly upon entry into the tidal sphere is given by

$$
f_{\mathrm{t}}=-\arccos (2 / \beta-1),
$$

then

$$
\begin{aligned}
& E=-\tilde{z}_{0} \sqrt{\beta-1} \\
& F=\tilde{z}_{0} .
\end{aligned}
$$

If we introduce a tidal potential $\Psi$ felt in the rest frame of the star, the tidal acceleration is given by SKR10 as

$$
\ddot{\tilde{z}}=-\frac{\partial \Psi}{\partial \tilde{z}}=-\beta^{3} \frac{(1+\cos f)^{3}}{8} \tilde{z} .
$$

We note that the self-similarity of Eq. 4.12 implies that the free solutions all collapse to $z=0$ simultaneously at a true anomaly $f_{\mathrm{c}}$, although physically this collapse will be reversed shortly before by the buildup of pressure gradients strong enough to counteract the tidal forces compressing the star. However, it is useful to solve for $f_{\mathrm{c}}$ using Eq. 4.8:

$$
\tan f_{\mathrm{c}}=\frac{1}{(\beta-1)^{1 / 2}} .
$$

From this formula we see that in the limit of $\beta \rightarrow \infty$, collapse along the z-axis occurs at $f_{\mathrm{c}}=0$, i.e. at pericenter, while in the marginal disruption limit of $\beta \rightarrow 1$, collapse occurs 


\section{CHAPTER 4. STRONG COMPRESSION IN TDES}

at $f_{\mathrm{c}}=\pi / 2$, i.e. at a fixed point past pericenter. We have already mentioned that the free solutions become less valid for small $\beta$ due to the increasing importance of internal forces, but we can see from Eq. 4.13 a second, stronger, inconsistency at low $\beta$, which is that the free solutions dictate vertical collapse after the disrupted star leaves the tidal sphere, i.e. $f_{\mathrm{c}}>\left|f_{\mathrm{t}}\right|$. This occurs for $\beta \lesssim 1.3$.

Although the onset of Phase III is dictated by compression in the $\hat{z}$ direction, the outcome of the bounce will be affected by motion within the orbital plane during Phase II, when $f<f_{\mathrm{c}}$. We therefore describe here the free solutions within the orbital plane (SKR10):

$$
\begin{array}{r}
\tilde{x}_{\mathrm{H}}=A \tilde{x}_{\mathrm{A}}+B \tilde{x}_{\mathrm{B}}+C \tilde{x}_{\mathrm{C}}+D \tilde{x}_{\mathrm{D}} \\
\tilde{y}_{\mathrm{H}}=A \tilde{y}_{\mathrm{A}}+B \tilde{y}_{\mathrm{B}}+C \tilde{y}_{\mathrm{C}}+D \tilde{y}_{\mathrm{D}} \\
\tilde{x}_{\mathrm{A}}=-\frac{1}{\beta} \frac{\sin f}{1+\cos f} \\
\tilde{y}_{\mathrm{A}}=\frac{1}{\beta} \frac{\cos f}{1+\cos f} \\
\tilde{x}_{\mathrm{B}}=-\frac{1}{\beta} \sin f \\
\tilde{y}_{\mathrm{B}}=\frac{1}{\beta}(1+\cos f) \\
\tilde{x}_{\mathrm{C}}=\frac{1}{\beta}(2-\cos f) \\
\tilde{y}_{\mathrm{C}}=-\frac{1}{\beta} \cos f \tan (f / 2) \\
\tilde{y}_{\mathrm{D}}=\frac{1}{\beta} \frac{35 \sin f-2 \sin (2 f)+3 \sin (3 f)}{(1+\cos f)^{2}}
\end{array}
$$

If we consider a point on the star with an initial position, relative to the star's center of mass, of $\left(x_{0}, y_{0}, z_{0}\right)$ and zero initial velocity (here, as before, "initial" refers to $f=f_{\mathrm{t}}$, 


\section{CHAPTER 4. STRONG COMPRESSION IN TDES}

i.e. crossing into the tidal sphere), then we have 4 initial conditions for 4 unknowns: $\{A, B, C, D\}$. Using Eq. 4.9, we find

$$
\begin{aligned}
A= & \frac{1}{\beta^{2}}\left(-8 \tilde{x}_{0} \sqrt{\beta-1}+2 \tilde{y}_{0}\left(\beta^{2}+2 \beta-4\right)\right) \\
B= & \frac{1}{5 \beta^{2}}\left(2 \tilde{x}_{0} \sqrt{\beta-1}\left(\beta^{3}-4 \beta^{2}+8\right)\right. \\
& \left.+\tilde{y}_{0}\left(9 \beta^{3}-12 \beta^{2}-8 \beta+16\right)\right) \\
C= & \frac{1}{\beta^{2}}\left(\tilde{x}_{0}\left(2 \beta^{2}+\beta-2\right)-2 \tilde{y}_{0} \sqrt{\beta-1}\left(\beta^{2}-1\right)\right) \\
D= & -\frac{1}{20 \beta^{2}}\left(\tilde{x}_{0}(\beta-2)+2 \tilde{y}_{0} \sqrt{\beta-1}\right)
\end{aligned}
$$

All six of the free solutions we have listed can be thought of as slight perturbations to a different orbital element of the parabolic center of mass trajectory, boosted into the center of mass frame. The free solutions represent freely falling particles in a Newtonian potential, but with a coordinate origin following a parabolic center of mass trajectory.

We have now exactly specified the motion of the idealized star's fluid elements in the orbital plane during Phase II. We plot the vertical free solutions for a variety of $\beta$ in Fig. 4.1, and snapshots from motion within the orbital plane in Fig. 4.2. Here we list several important features of the free solutions, when they are initialized with static spheres of matter at $f=f_{\mathrm{t}}$ :

- For $f>f_{\mathrm{t}}$, an initially spherical shell of matter will deform into a sequence of roughly ellipsoidal shapes. It is simple to demonstrate that they do not generally take the form of true ellipsoids, however.

- Initially concentric spherical shells of matter remain concentric, with in-plane principal axes that remain aligned with those of other concentric shells. 
CHAPTER 4. STRONG COMPRESSION IN TDES

- Slices of the star through the orbital plane $(\mathrm{z}=0)$ maintain reflection symmetry across their rotating in-plane principal axes.

- The derivation of the free solutions assumes that $R_{*} / R \ll 1$ (SKR10). If we neglect stretching of the star, this is equivalent to requiring $\beta \ll\left(M_{\mathrm{BH}} / M_{*}\right)^{1 / 3}$, a condition that is in general easily satisfied: a $10^{6} M_{\odot} \mathrm{SMBH}$, if non-spinning, cannot disrupt solar-type stars with $\beta \gtrsim 11$ (higher $\beta$ values will place the pericenter interior to the marginally bound circular orbit, and the star will plunge directly into the horizon). Even a maximally spinning SMBH of this mass cannot disrupt solar-type stars with $\beta \gtrsim 47$. The effects of tidal stretching will make it somewhat harder to satisfy this assumption, but only for the minority of the star's mass that is strongly stretched. 


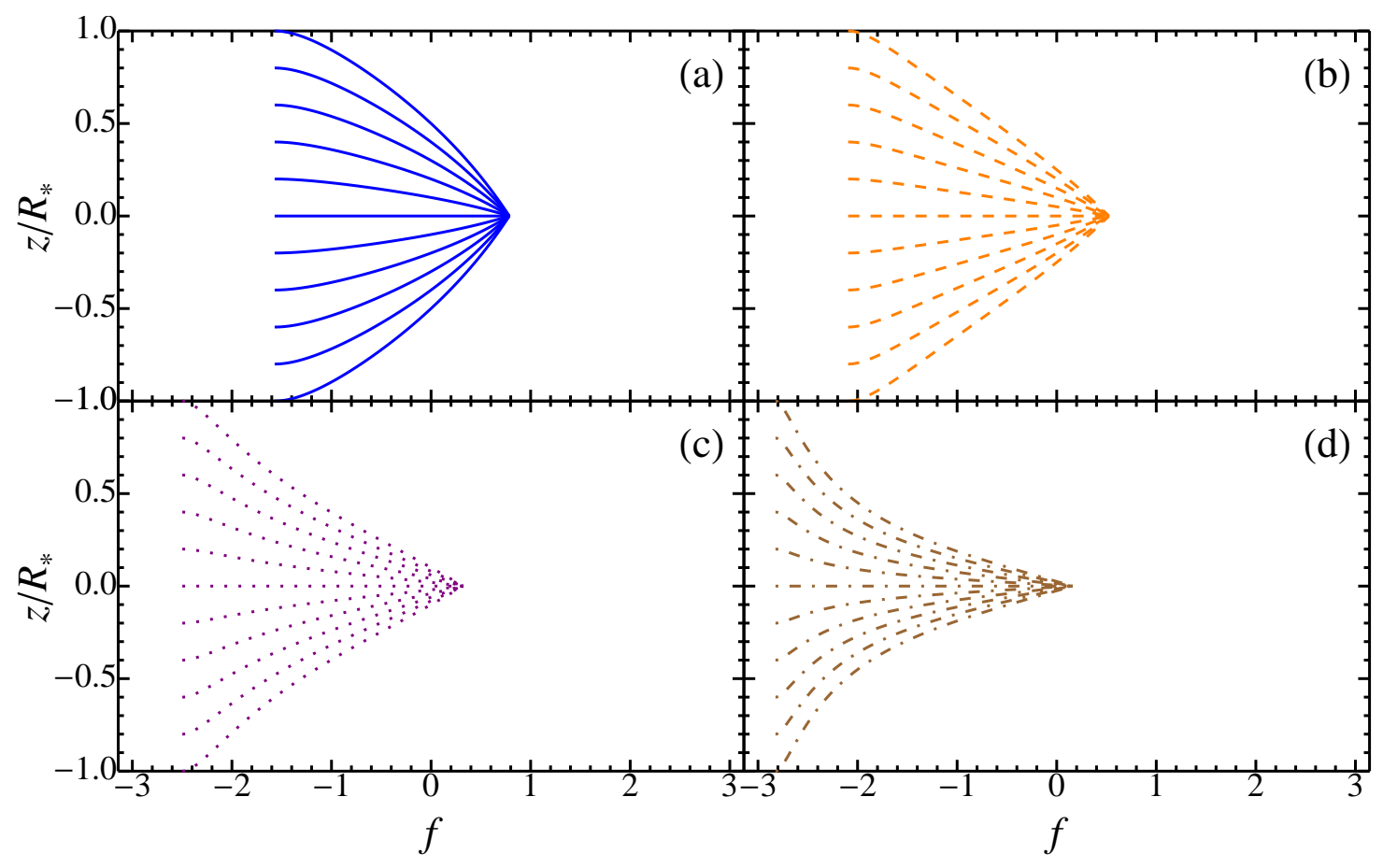

Figure 4.1.-: Normalized height $\tilde{z}=z / R_{*}$ versus true anomaly $f$ for the vertical collapse of one-dimensional stars at varying $\beta$. The solid blue curves in panel (a) are $\beta=2$; the dashed orange curves in panel (b) are $\beta=4$; the dotted purple curves in panel (c) are $\beta=10$; the dot-dashed brown curves in panel (d) are $\beta=40$. Each scenario is initialized at $f=f_{\mathrm{t}}(\beta)$. Note that $f=0$ corresponds to pericenter. 

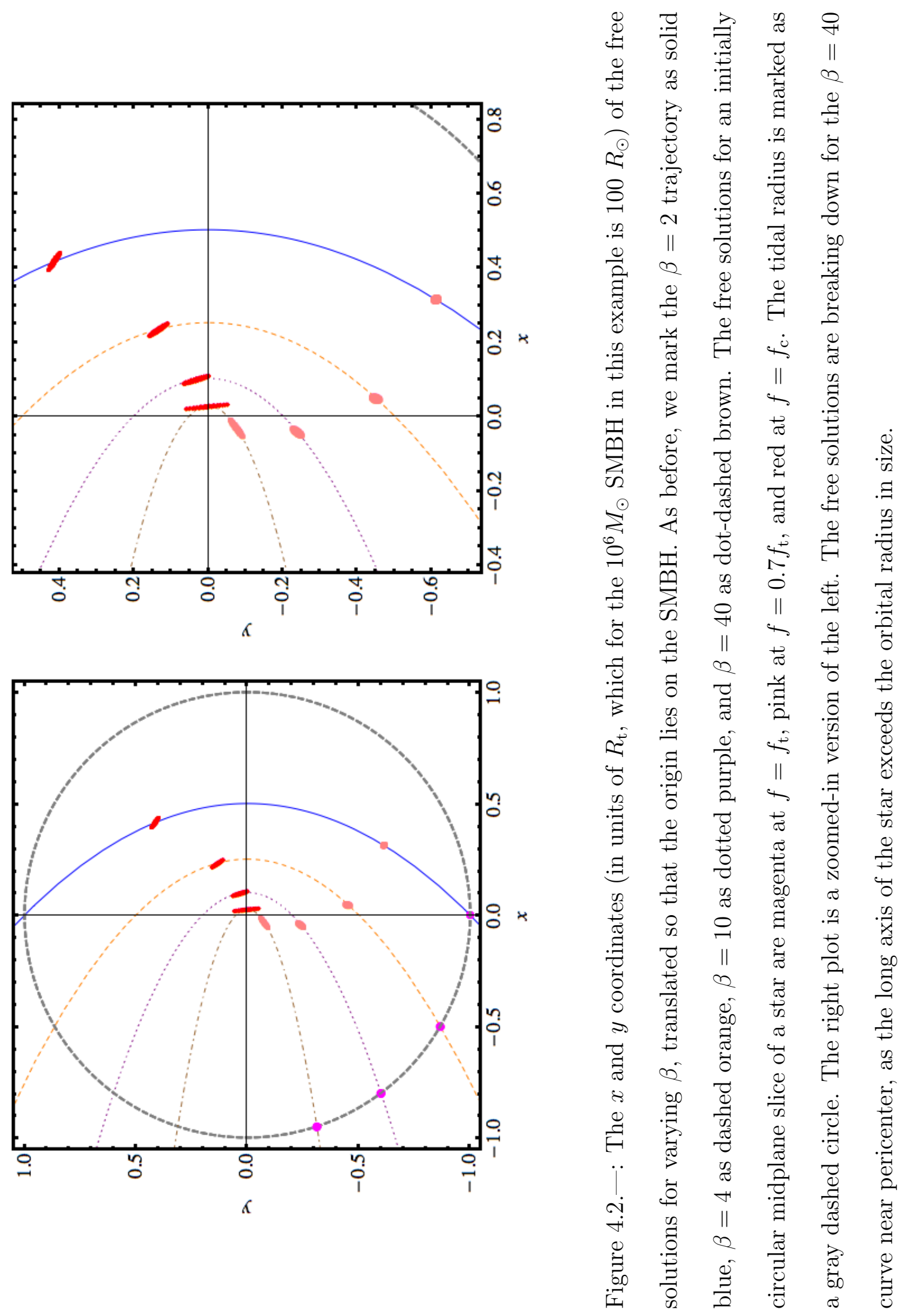


\section{CHAPTER 4. STRONG COMPRESSION IN TDES}

The free solutions allow us to directly solve for the stellar axis ratio as a function of $f$ or $t$, and it is trivial to do so numerically, but there is an exact analytic solution as well. If we denote the lengths of the long and short principal axes of our tidally distorted star (within the orbital plane) as $r_{\text {long }}$ and $r_{\text {short }}$, respectively, we can solve for them by rewriting $\tilde{x}_{0}=\cos \theta_{0}, \tilde{y}_{0}=\sin \theta_{0}$, and finding the appropriate $\theta_{0}$. More specifically, we set $\frac{\mathrm{d}}{\mathrm{d} \theta} R_{\mathrm{H}}^{2}(f)=0$ (with $R_{\mathrm{H}}^{2}=x_{\mathrm{H}}^{2}+y_{\mathrm{H}}^{2}$ ), and solve for $\theta_{\mathrm{ex}}$, the values of $\theta_{0}$ which extremize $R_{\mathrm{H}}$. More physically, we are searching for the initial angles $\theta_{\mathrm{ex}}$ around the star which at a later orbital phase $f$ will correspond to its principal axes in the orbital plane. Once we have the initial angular positions of the principal axes, $\theta_{\mathrm{ex}}$, we can plug in to Eqs. (4.14) and solve for the size of the principal axes at a later true anomaly $f>f_{\mathrm{t}}$. We also find the misalignment angle $\nu$ between the long in-plane principal axis and the orbital velocity vector. The in-plane stellar geometry is presented in Fig. 4.3.

The algebra involved in this solution is unenlightening, so we leave the general solution $\theta_{\mathrm{ex}}(f)$ for numerical work and only derive analytic expressions for $\theta_{\mathrm{ex}}\left(f_{\mathrm{c}}\right)$, which is the situation of greatest interest. The details are contained in Appendix A, but we plot the results below in Fig. 4.4. Specifically, these are the sizes of the principal axes at $f=f_{\mathrm{c}}$. For comparison we plot curves of the high $\beta$ limiting behavior, for which $\tilde{r}_{\text {long }} \approx \frac{4}{5} \beta^{1 / 2}+\frac{22}{5} \beta^{-1 / 2}$ and $\tilde{r}_{\text {short }} \approx 2 \beta^{-1 / 2}-\frac{23}{2} \beta^{-3 / 2}$.

The primary interesting feature of the axis ratio calculations is that for disruptions of stars by supermassive black holes, the physically relevant range of $r_{\text {long }}$ and $r_{\text {short }}$ is quite narrow, being confined between 3 to 5 for the former, and 0.3 to 0.5 for the latter. For the tidal disruption of a star by an intermediate mass black hole (IMBH), or

perhaps more exotic disruption scenarios, a larger range of $\beta$ (and therefore $r_{\text {long }}, r_{\text {short }}$ ) can be attained, but for star-SMBH TDEs only a surprisingly narrow range of principal 


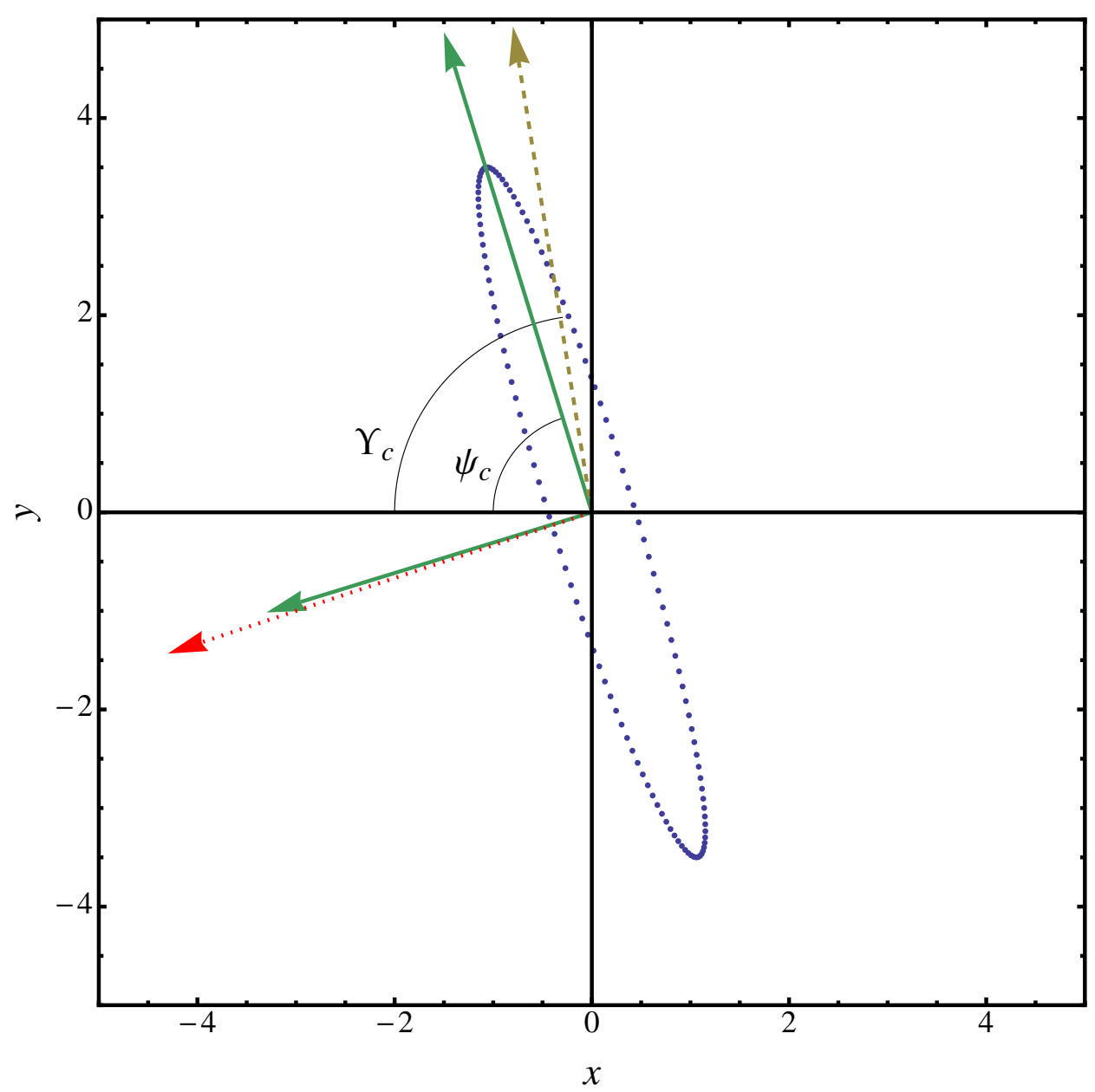

Figure 4.3.- : An initially $\left(f=f_{\mathrm{t}}\right)$ circular ring of stellar fluid elements has been tidally distorted by the time it reaches $f=f_{\mathrm{c}}$. The principal axes of the distorted, free-falling body are the solid green vectors, the center of mass velocity is the dashed yellow vector, and the direction to the SMBH is the dotted red vector. The angle $\psi_{\mathrm{c}}\left(\Upsilon_{\mathrm{c}}\right)$ is measured between the negative $\hat{x}$ direction and the long principal axis (stellar velocity vector). We define the misalignment angle $\nu_{\mathrm{c}}=\Upsilon_{\mathrm{c}}-\psi_{\mathrm{c}}$. 
CHAPTER 4. STRONG COMPRESSION IN TDES

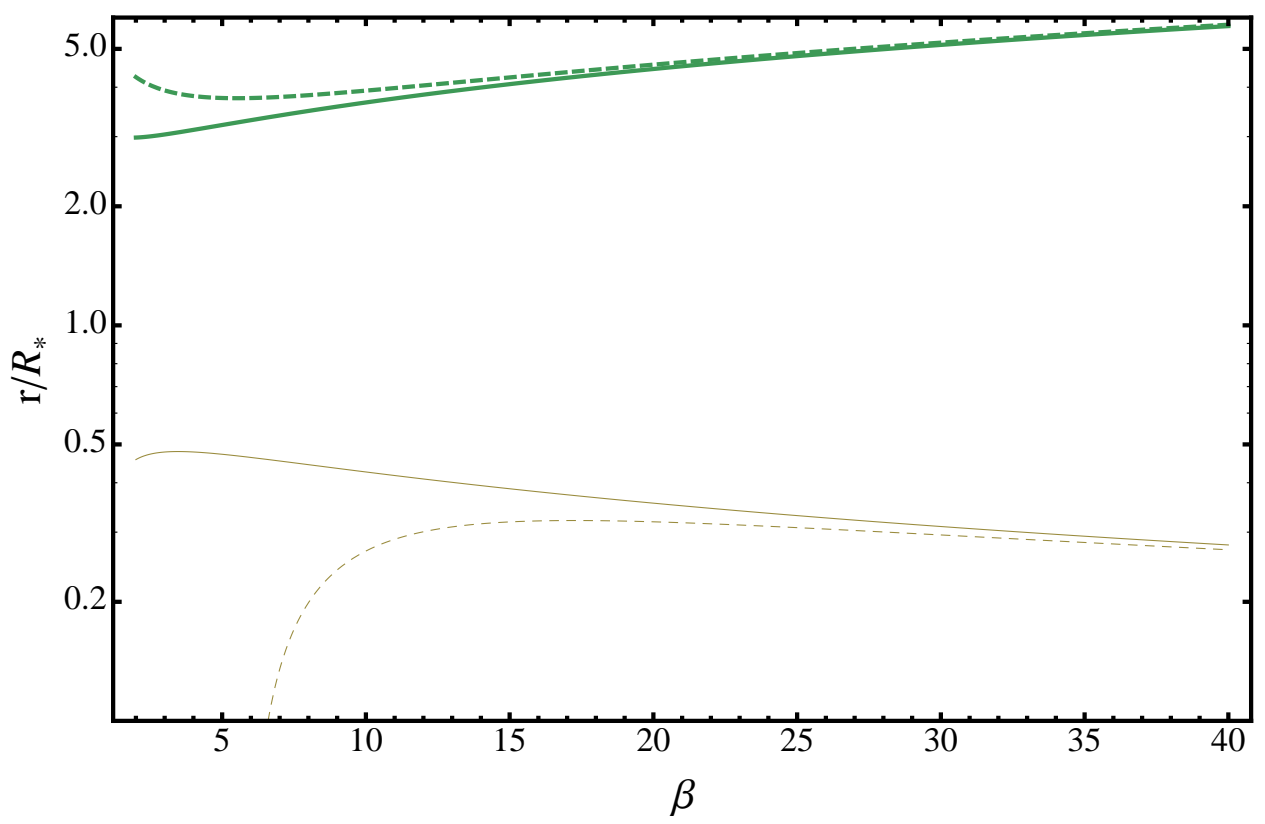

Figure 4.4.- : The principal axis lengths, $r_{\text {long }}$ and $r_{\text {short }}$, of the distorted star (at $f=f_{\mathrm{c}}$ ) vs $\beta$. Here $r_{\text {long }}$ is plotted as thick green curves; $r_{\text {short }}$ as thin yellow curves. The exact solutions are solid lines, while the dashed curves are the high- $\beta$ Taylor expansions given by Eqs. (B.6), (B.7). 


\section{CHAPTER 4. STRONG COMPRESSION IN TDES}

axis lengths are accessible. This implies that the naive Taylor expansion of the SMBH potential as the star passes through $R=R_{\mathrm{p}}$, i.e. Eq. 4.2 , will fail primarily because of internal velocities within the free-falling stellar debris, and only secondarily because of distortions in the star's shape.

As we shall see in $\S 4$, when estimating energy redistribution during maximum vertical compression, the misalignment angle $\nu$ plays a larger role than the slowly-varying axis ratio. This will prove relevant when calculating corrections to $\Delta \epsilon$, and is shown in Fig. 4.5. The angle $\nu_{\mathrm{c}}$ (as elsewhere, the subscript c denotes evaluation at $f=f_{\mathrm{c}}$ ) is found to be a rapidly decreasing function of $\beta$; to leading order, $\tan \nu_{\mathrm{c}} \propto \beta^{-3 / 2}$.

We can now use the free solutions $\{A, B, C, D, E, F\}$ to quantify precisely the spread in debris energy at the tidal radius. Because these solutions can be thought of physically as perturbations to the orbital elements of a parabolic trajectory, all possess exactly zero energy except for the in-plane "D" solution, which has specific energy given by

$$
\epsilon=-\frac{20 G M_{*} D}{R_{*}}\left(\frac{M_{\mathrm{BH}}}{M_{*}}\right)^{1 / 3} \beta,
$$

where $D$ is the coefficient of the fourth in-plane free solution, corresponding to slight

variations in the eccentricity of a near-parabolic orbit (SKR10). If we initialize our free solutions with an unperturbed sphere, i.e. Eqs. 4.15, then we find a specific energy for each fluid element of

$$
\epsilon_{u}=\frac{G M_{\mathrm{BH}} R_{*}}{R_{\mathrm{t}}^{2}}\left(\tilde{x}_{0}(1-2 / \beta)+2 \tilde{y}_{0} \sqrt{\beta^{-1}-\beta^{-2}}\right),
$$

where $\tilde{x}_{0}$ and $\tilde{y}_{0}$ are the initial positions of a debris stream relative to the star's center of mass at $R=R_{\mathrm{t}}$ normalized by the stellar radius. Notably, the specific energy is to leading order independent of $\beta$, with the weak $\beta$-dependence becoming negligible at high penetration factors. Defining $\tilde{x}_{0}=\tilde{r}_{0} \cos \theta_{0}$ and $\tilde{y}_{0}=\tilde{r}_{0} \sin \theta_{0}\left(0 \leq \tilde{r}_{0} \leq 1\right.$ 


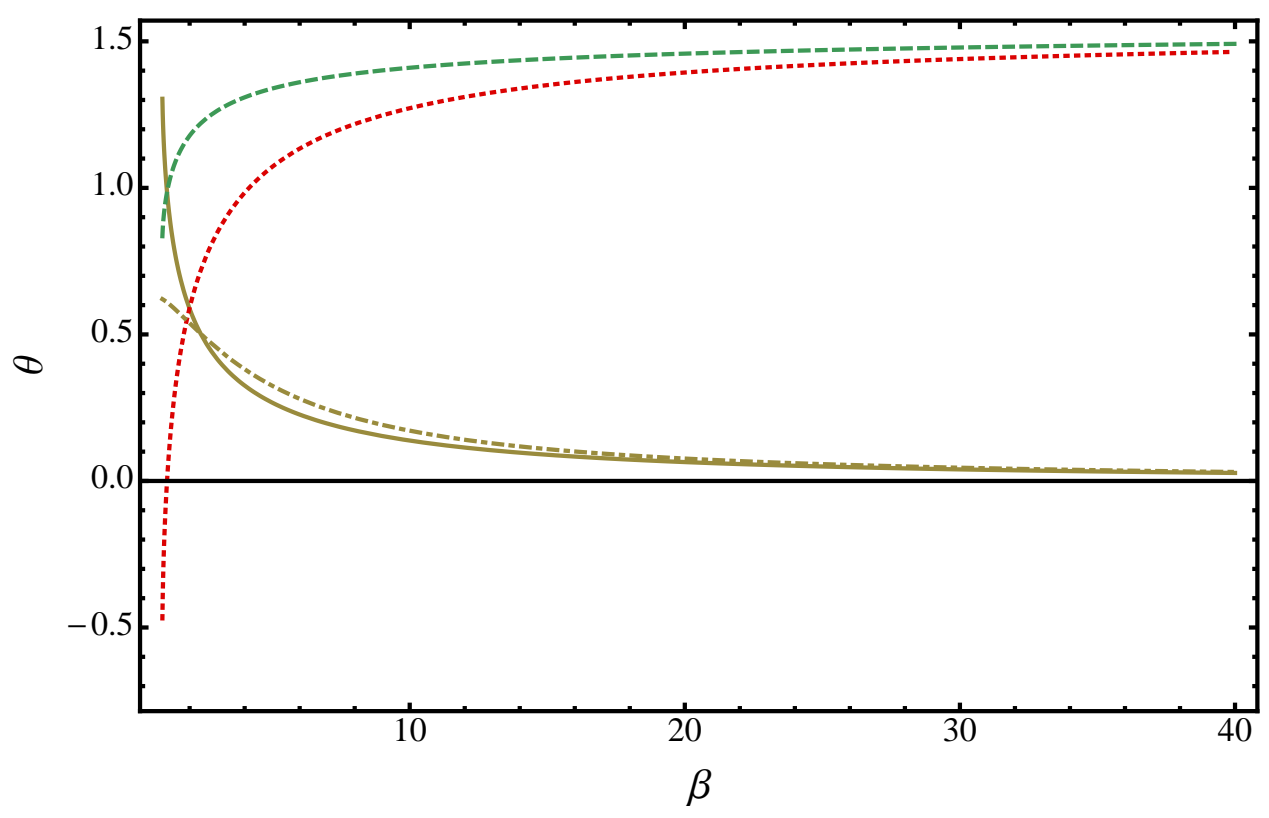

Figure 4.5.- : Curves illustrating misalignment of the tidally raised bulge and the orbital velocity vector at the point of z-collapse $\left(f=f_{\mathrm{c}}\right)$. The red dotted curve is the angle between the negative $\mathrm{x}$-axis and the tidal bulge $\left(\psi_{\mathrm{c}}\right)$, the green dashed curve is the angle between the negative $\mathrm{x}$-axis and the orbital velocity vector, and the solid yellow curve is the difference between them, i.e. the misalignment angle $\left(\nu_{\mathrm{c}}\right)$. These angles are plotted against the penetration factor $\beta$. The high $\beta$ limit for $\nu_{\mathrm{c}}$ is the dot-dashed yellow curve. 


\section{CHAPTER 4. STRONG COMPRESSION IN TDES}

is the initial internal radius, and $0 \leq \theta_{0}<2 \pi$ is the initial azimuthal angle), we can analytically extremize Eq. 4.20 with respect to $\theta_{0}$, to find that the spread in energy of these unperturbed free solutions is actually fully independent of $\beta$ :

$$
\Delta \epsilon_{\mathrm{u}}=\frac{2 G M_{\mathrm{BH}} R_{*}}{R_{\mathrm{t}}^{2}} .
$$

In our idealized model of a spherical, stationary star undergoing tidal free fall, the assumption of energy freeze-in at the moment of disruption implies $n=0$, and no $\beta$ dependence in $\Delta \epsilon_{\mathrm{u}}$. This result reflects an assumption of our model: by imposing energy freeze-in at the tidal radius, the energy spread of the debris will simply be the potential spread across the star at that point. This picture is complicated slightly if our initial conditions become more general, and in particular a weak $\beta$ dependence, below leading order, can be reintroduced for spinning stars (see §5.3).

Energy freeze-in can be understood geometrically: curves of constant specific energy across the test particles of a spherical (static) star are lines with slope $\tilde{y}_{0} / \tilde{x}_{0}=(2-\beta) /(2 \sqrt{\beta-1})$. Since this slope $\tilde{y}_{0} / \tilde{x}_{0}=\cot f_{\mathrm{t}}$, these lines are orthogonal to $\hat{X}\left(f=f_{\mathrm{t}}\right)$, and the zero-energy $\mathrm{D}=0$ line is the one passing through the center of the star. On one side of that line, stellar matter is closer to the SMBH and remains gravitationally bound; the half of the star on the other side of the line is unbound.

In the following three sections, we examine the robustness of this model, and consider possible corrections to our expressions for $\Delta \epsilon$. With limited exceptions, we find that the arguments made in this section remain generally valid. 
CHAPTER 4. STRONG COMPRESSION IN TDES

\subsection{Total Vertical Collapse and Bounce}

For $f \approx f_{\mathrm{c}}$, motion in the vertical direction has decoupled from in-plane motion and the star undergoes a homologous vertical collapse. In this regime, the vertical velocity of the free solutions near the point of maximum collapse is very close to a constant value, with $w_{\mathrm{c}} \propto \beta$, a result known since CL83, although the exact value, for arbitrary $f_{\mathrm{c}}$, is

$$
w_{\mathrm{c}}=\beta \tilde{z}_{0}\left(\frac{G M_{*}}{2 R_{*}}\right)^{1 / 2}\left(\left(1-\beta^{-1}\right)^{1 / 2}+1\right) .
$$

With this formula we can begin thinking about Phase III of a tidal disruption event, and in particular whether it can alter Eq. 4.21.

As we have seen in the previous section, once $f \approx f_{\mathrm{c}}$, the majority of the star simultaneously "pancakes" into a sheet of matter strongly compressed in the vertical direction. If non-gravitational forces were truly negligible, an idealized, one-dimensional ( $\hat{z}$ extent only) star would momentarily possess zero height at $f=f_{\mathrm{c}}$, but in reality sufficient compression will create a pressure gradient strong enough to oppose free fall in the z-direction. The resulting bounce will reverse the vertical free fall and lead to vertical expansion at speeds comparable to $w_{\mathrm{c}}$. The vertical rebound will have a limited impact on $\Delta \epsilon$ because it is effectively decelerated by the tidal potential (for example, if we generously approximate the rebound as elastic due by reflecting $w$ at $f=f_{\mathrm{c}}$, the asymptotic free solution velocity $w \rightarrow 0$ as $f \rightarrow \pi$ ), but the smaller rebound velocities $\Delta v_{\mathrm{x}}, \Delta v_{\mathrm{y}}$ in the orbital plane can in principle have more significant effects, as $\Delta \epsilon \sim V_{\mathrm{p}} \Delta v$. In this section we assume the bounce is adiabatic; in particular, we neglect both dissipation in shocks and the thermonuclear energy release from the compression of the stellar core. For a more thorough discussion of these possibilities see Luminet \& Pichon (1989); Brassart \& Luminet (2008). 


\section{CHAPTER 4. STRONG COMPRESSION IN TDES}

During Phase III of a TDE, the requirement that central pressure rises to halt the kinetic energy of collapse $\left(\sim M_{*} w_{\mathrm{c}}^{2}\right)$ implies that the star's peak internal specific energy will be

$$
U_{\mathrm{c}} \sim \beta^{2} U_{*}\left(\sqrt{1-\beta^{-1}}+1\right)^{2}
$$

where $U_{*}=G M_{*} / R_{*}$. Assuming a polytropic equation of state $P=K \rho^{\gamma}$, and furthermore that strong compression in the $\hat{z}$ direction means that the density enhancement will be due to collapse in $\hat{z}$ alone (since the cross-sectional area within the orbital plane, $\approx \pi r_{\text {long }} r_{\text {short }}$ remains roughly constant), gives a minimum stellar height and maximum stellar density of

$$
\frac{z_{\min }}{R_{*}} \sim \frac{\rho_{*}}{\rho_{\mathrm{c}}} \sim \beta^{-2 /(\gamma-1)},
$$

where $\rho_{*}$ is the mean pre-disruption stellar density. The duration of maximum compression will be roughly $\tau_{\mathrm{c}} \sim w_{\mathrm{c}} /\left(R_{*}^{2} P_{\mathrm{c}}\right)$. This yields a steep power of the impact parameter, specifically

$$
\tau_{\mathrm{c}} \sim \beta^{-(\gamma+1) /(\gamma-1)} \tau_{*},
$$

with $\tau_{*}=1 / \sqrt{G \rho_{*}}$. Although we have only derived these formulas at the order of magnitude level, they have been calibrated over a wide range of $\beta$ by both the affine model (Luminet \& Carter 1986) and one-dimensional hydrodynamical simulations (Brassart \& Luminet 2008) (hereafter BL08). Specifically, for $\gamma=5 / 3$ polytropes, the affine model found $U_{\mathrm{c}} \approx 1.2 U_{*} \beta^{2}, \rho_{\mathrm{c}}=1.3 \rho_{*} \beta^{3}$ and $\tau_{\mathrm{c}}=8.5 \tau_{*} \beta^{-4}$, calibrations which were essentially duplicated in BL08. Likewise, $z_{\min } \approx 4.5 \beta^{-3} R_{*}$ if we assume that the rise in density comes entirely from homologous, vertical stellar collapse (i.e. that the in-plane area of the compressed star is $\left.\sim r_{\text {long }} r_{\text {short }}\right)$.

If we assume that the pressure-driven bounce acts isotropically (i.e. that shear 


\section{CHAPTER 4. STRONG COMPRESSION IN TDES}

stresses from viscosity or shocks remain unimportant), then the relevant changes in velocity can be estimated as $\rho_{\mathrm{c}} \Delta v_{\mathrm{i}} / \tau_{\mathrm{c}} \sim \Delta P_{\mathrm{c}} / r_{\mathrm{i}}$, with $r_{\mathrm{i}}$ the physical dimension of the star parallel to the pressure gradients that impart $\Delta v_{\mathrm{i}}$. Specifically,

$$
\begin{aligned}
\Delta v_{\mathrm{z}} & \sim \sqrt{U_{\mathrm{c}}} \frac{z_{\text {min }}}{z_{\text {min }}} \\
\Delta \vec{v}_{\text {short }} \cdot \hat{V}_{\mathrm{c}} & \sim \sqrt{U_{\mathrm{c}}} \frac{z_{\text {min }}}{r_{\text {short }}} \sin \left(\nu_{\mathrm{c}}\right) \\
\Delta \vec{v}_{\text {long }} \cdot \hat{V}_{\mathrm{c}} & \sim \sqrt{U_{\mathrm{c}}} \frac{z_{\text {min }}}{r_{\text {long }}} \cos \left(\nu_{\mathrm{c}}\right),
\end{aligned}
$$

where we have denoted the direction of the orbital velocity at $f=f_{\mathrm{c}}$ as the dimensionless unit vector $\hat{V}_{\mathrm{c}}$. As $\beta$ increases, the increasing central pressure would enhance the in-plane velocity perturbations, but is counterbalanced by the increasingly extreme compression of the star, i.e. the increasing ratio of the vertical pressure gradient to in-plane pressure gradients. The latter factor win out, and the velocity perturbations decrease with increasing $\beta$.

This leads to energy perturbations within the orbital plane at bounce of $\Delta \epsilon_{\mathrm{III}} \sim V_{\mathrm{c}} \Delta v$. Using our exact formulae for axis lengths and alignment, we plot the results in Fig. 4.6, along with the limiting behavior at high $\beta$, which is well-approximated by the Taylor expansions in Appendix A as:

$$
\begin{array}{r}
\Delta \epsilon_{\mathrm{III}, \text { short }} \sim 31 \beta^{-5 / 2} \Delta \epsilon_{\mathrm{u}} \\
\Delta \epsilon_{\mathrm{III}, \text { long }} \sim 9 \beta^{-2}\left(1+\frac{11}{2 \beta}\right)^{-1} \Delta \epsilon_{\mathrm{u}} .
\end{array}
$$

Here we have assumed $\gamma=5 / 3$, and that $z_{\text {min }} \approx 5 R_{*} \beta^{-3}$ based on the BL08 calibration. We have also approximated $V_{\mathrm{c}} \approx V_{\mathrm{p}}$, which is accurate for high $\beta$ though a mild overestimate at low $\beta$. Even with this overestimate, we can see from Fig. 4.6 that only for $\beta \lesssim 3$ (where our model's assumption of tidal free fall begins to break down) can the 


\section{CHAPTER 4. STRONG COMPRESSION IN TDES}

pressure-driven bounce along the short principal axis of the star provide an order unity enhancement to the total spread in (in-plane) debris energy. The contribution of the bounce along the longer principal axis remains negligible at all $\beta$.

Because the z-component of $\vec{V}_{\mathrm{c}}=0$, the spread in kinetic energy of vertical motion at the time of bounce is given by

$$
\Delta \epsilon_{\mathrm{III}, \mathrm{z}} \sim \Delta v_{\mathrm{z}}^{2} \sim \beta^{2} \frac{G M_{*}}{R_{*}} .
$$

Interestingly, for $\beta \gtrsim 10$ the total spread in kinetic energy at the time of bounce is dominated by $\Delta \epsilon_{\mathrm{III}, \mathrm{z}}$, not $\Delta \epsilon_{\mathrm{u}}$. However, the instantaneous vertical kinetic energy at $f=f_{\mathrm{c}}$ will disappear as $f \rightarrow \pi$. Even neglecting dissipation of the kinetic energy of vertical free fall into shocks, and assuming a perfectly elastic bounce, the tidal potential (Eq. 4.12) will efficiently decelerate the vertical motion of the debris during the phase IV rebound and later expansion. This can be seen by continuing the free solutions past $f=f_{\mathrm{c}}$, which corresponds to a reflection of vertical velocity.

Here we have ignored energy release from thermonuclear burning at the time of maximum compression, which in principle could increase $\Delta \epsilon$. However, past estimates made in the framework of the affine model (Luminet \& Pichon 1989, Table 8) found that for the range of $\beta$ values considered $(5 \leq \beta \leq 20)$, the total thermonuclear energy release was less than $U_{\mathrm{c}}$, making it unlikely to change the analysis of this section.

Of course, our analysis of energy redistribution in Phase III depends critically on how synchronously the vertical collapse of the star proceeds. If individual "columns" of the star do not collapse in the homologous manner implied by Eq. 4.12, it is unlikely $z_{\text {min }}$ will reach the extreme values predicted by the simple arguments in this section. Alternatively, if separate columns collapse in a desynchronized way, it is possible that 


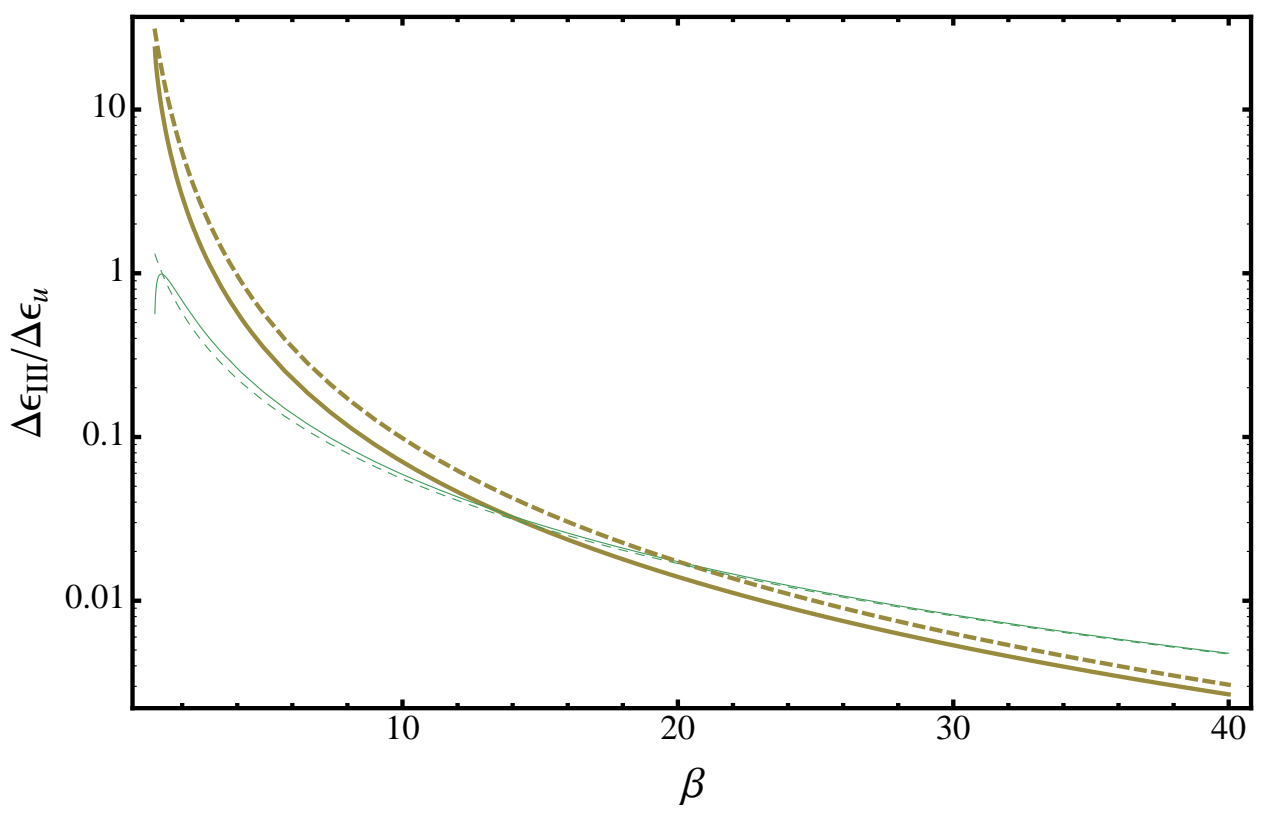

Figure 4.6.- : Fractional specific energy perturbations during the bounce phase. We plot perturbations along the short axis of the star as thick yellow curves, and along the short axis as thin green curves. Exact solutions are solid lines, and the leading order behavior from Eqs. (4.27) and (4.28) are dashed lines. The bounce represents at most a factor $\approx 2$ correction to specific energy of the stellar debris for $4 \gtrsim \beta \gtrsim 3$; above these values, the bounce is negligible. Below $\beta \approx 3$, a larger correction is possible, but the free solutions become somewhat unreliable. The high degree of alignment between the stellar bulge and the orbital velocity vector causes perturbations along the long axis to actually dominate those along the short axis above $\beta \approx 15$. 


\section{CHAPTER 4. STRONG COMPRESSION IN TDES}

pressure waves from collapsed regions of the star will propagate upstream to uncollapsed regions and cause them to rebound prematurely. In either scenario, the effective $z_{\text {min }}$ will be enhanced, enabling greater coupling of the bounce energy to motions within the orbital plane, and increasing the values of $\Delta \epsilon_{\mathrm{III}, \text { short }}$ and $\Delta \epsilon_{\mathrm{III}, \mathrm{long}}$. Therefore, Eq. 4.21 should be regarded as a lower bound on $\Delta \epsilon$ - a higher value of $n$ would be favored if the desynchronization of vertical collapse transfers kinetic energy to in-plane motions more efficiently than in our estimates here. A similar effect should arise in hydrodynamical simulations of TDEs that lack sufficient vertical resolution to capture the maximum compression of the star (we discuss this further in $\S 9$; see also Guillochon et al. 2009). In the following section, we consider physical sources of desynchronization.

\subsection{Desynchronization}

The synchronous vertical collapse of a one-dimensional star into a thin, pancake-like sheet only occurs if the initial distribution of vertical velocities is self-similar, i.e. the initial vertical velocity $w_{0}\left(z_{0}\right) \propto z_{0}$. In previous sections we have assumed the trivial self-similarity of $w_{0}=0$. Deviations from self-similarity will be seeded at early times by the nonlinear hydrodynamics of actual disruption at the tidal radius $R_{\mathrm{t}}$, and also later, as the self-gravity and pressure of the stellar debris perturbs the free solutions for $f<f_{\mathrm{c}}$. In this section we quantify in an approximate way the effect of desynchronization on our idealized earlier conclusions, finding that both the stellar properties during Phase III (important for any shock breakout signal) as well as $\Delta \epsilon$ could be significantly altered. However, we then argue that past hydrodynamical simulations indicate that desynchronization is likely to be suppressed in physical TDEs, justifying our use of the 
CHAPTER 4. STRONG COMPRESSION IN TDES

parabolic free solutions. Finally, we consider the desynchronization of stellar collapse in three dimensions.

\subsubsection{Desynchronized Free Solutions}

At $f=f_{\mathrm{t}}$, during the transition from Phase I to Phase II, velocity perturbations of order $\sim \sqrt{G M_{*} / R_{*}}$ could be imprinted on the free-falling stellar debris. Normalizing our initial conditions $\left\{z_{0}, w_{0}\right\}$ in units of $R_{*}$ and $\sqrt{G M_{*} / R_{*}}$, we derive coefficients for the "perturbed" (i.e. $w_{0} \neq 0$ ) vertical free solutions to be

$$
\begin{aligned}
& E_{\mathrm{p}}=-\tilde{z}_{0} \sqrt{\beta-1}-\tilde{w}_{0} \sqrt{\frac{1}{2 \beta}}(\beta-2) \\
& F_{\mathrm{p}}=\tilde{z}_{0}+\tilde{w}_{0} \sqrt{\frac{2}{\beta}} \sqrt{\beta-1}
\end{aligned}
$$

Therefore the true anomaly of a perturbed vertical collapse to $z=0$ is

$$
\tan \left(f_{\mathrm{c}}^{\prime}\right)=\frac{\tilde{z}_{0}+\tilde{w}_{0} \sqrt{2} \sqrt{\beta-1} / \sqrt{\beta}}{\tilde{z}_{0} \sqrt{\beta-1}+\tilde{w}_{0} \sqrt{2}(\beta / 2-1) / \sqrt{\beta}} .
$$

We note that both $f_{\mathrm{c}}$ and $f_{\mathrm{c}}^{\prime}$ go $\propto \beta^{-1 / 2}$ in the large $\beta$ limit. Unless $w_{0} \propto z_{0}$, the collapse will be non-homologous, with $f_{\mathrm{c}}^{\prime}$ depending on $z_{0}$. Modest deviations from homologous initial conditions will desynchronize the collapse, which we illustrate by plotting the desynchronized free solutions for $\beta=2$ and $\beta=10$. We can see that in both cases, the time at which the free solutions cross the orbital plane becomes strongly desynchronized, which complicates our previously simple treatment of the transition from "tidal free fall" to "pressure-driven bounce" and also raises the possibility that the vertical kinetic energy of free fall could be effectively isotropized and transferred to motions within the orbital plane, restoring a $\beta$ dependence to $\Delta \epsilon$.

We can estimate the amount of desynchronization by using trigonometric identities 


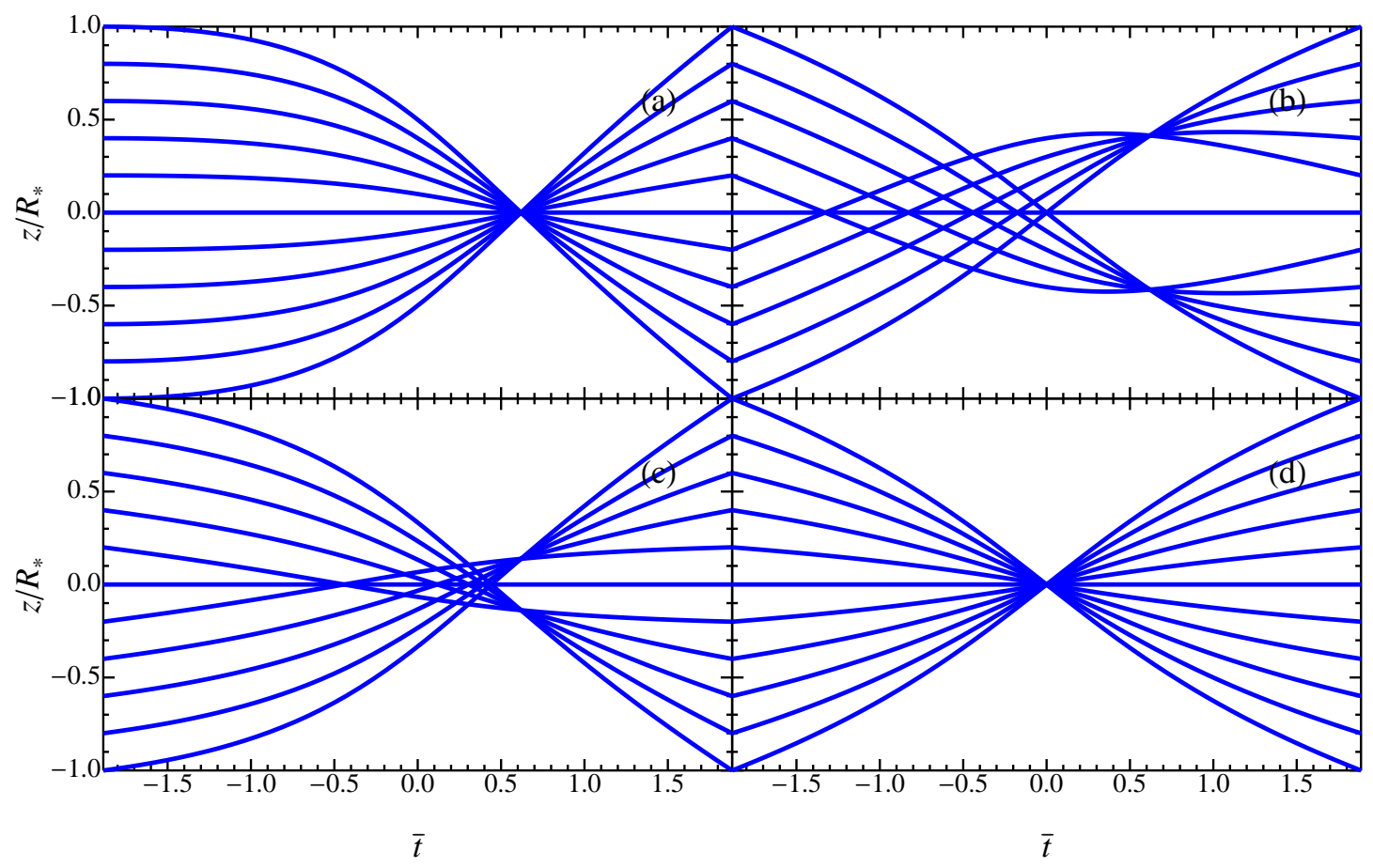

Figure 4.7.- : Here we plot several sets of free solutions $(\tilde{z}$ versus $\bar{t})$ for $\beta=2$. In panel a, the initial vertical velocity $w_{0}=0$. In panel $\mathrm{b}$, all fluid elements in the star receive initial velocity perturbations $\left|w_{0}\right|=\sqrt{G M_{*} / R_{*}}$; in panel c, $\left|w_{0}\right|=\sqrt{G M_{*} / R_{*}} / 3$. In panel $\mathrm{d}$, the star receives homologous velocity perturbations $w_{0}=-\tilde{z}_{0} \sqrt{G M_{*} / R_{*}}$, causing synchronous collapse before pericenter passage. 
CHAPTER 4. STRONG COMPRESSION IN TDES

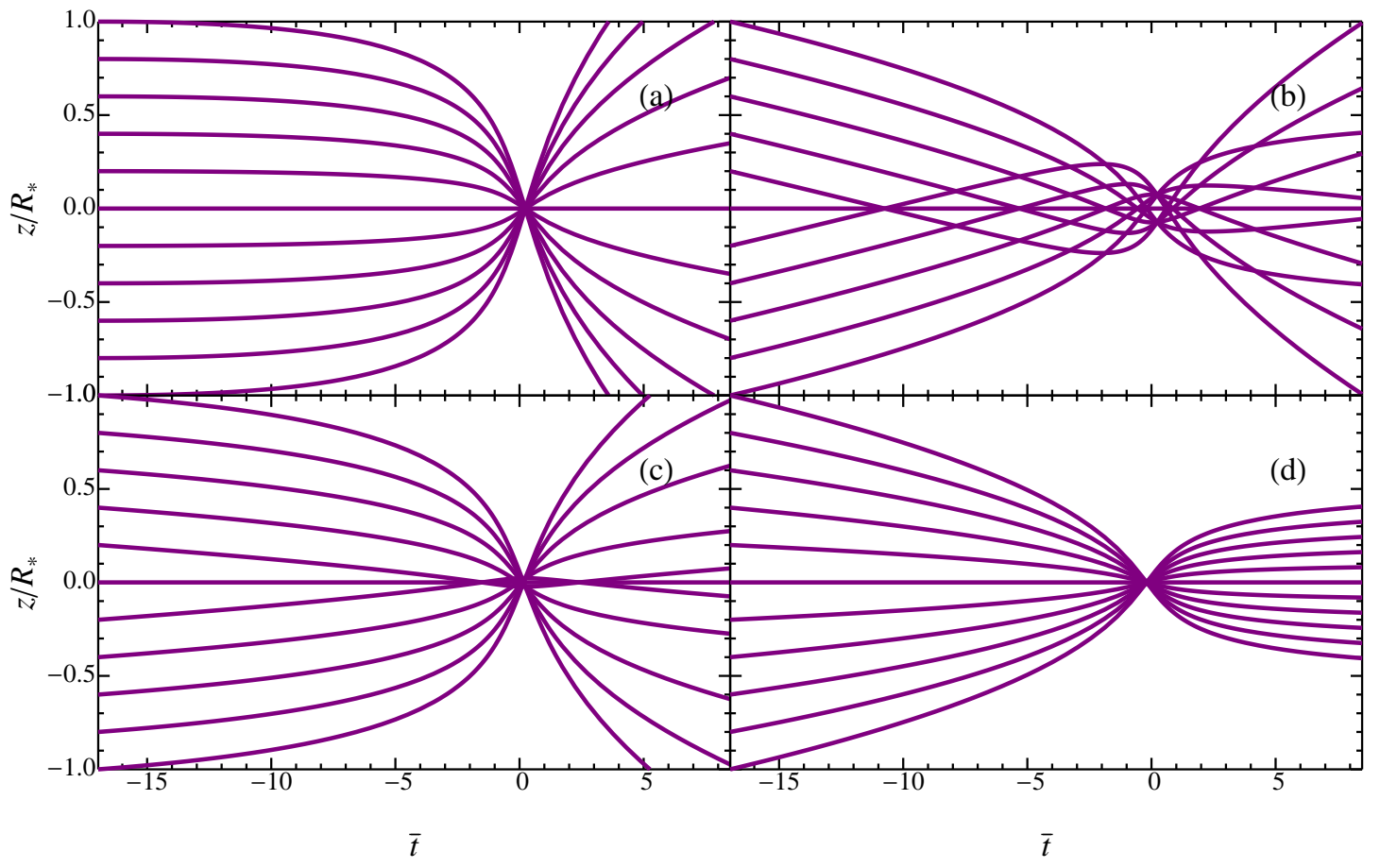

Figure 4.8.- : The same as Fig. 4.7, but for $\beta=10$. Desynchronization is less severe at higher $\beta$. 
and Eqs. 4.13 and 4.32 to find $\Delta f=f_{\mathrm{c}}^{\prime}-f_{\mathrm{c}}$. Specifically,

$$
\tan (\Delta f)=\frac{\tilde{\lambda}_{0}}{\sqrt{2 \beta}+\tilde{\lambda}_{0} \sqrt{\beta-1}}
$$

where $\tilde{\lambda}_{0}=\tilde{w}_{0} / \tilde{z_{0}}$.

Interestingly, both Figs. 4.7 and 4.8 show that most of the star's desynchronized free solutions have two crossings of the orbital plane, raising the possibility of a double bounce in desynchronized collapse scenarios (something previously seen only due to GR effects, e.g. Luminet \& Marck (1985) - see §VI). But is it realistic to expect desynchronized collapse?

\subsubsection{Validity of Free Solutions in One Dimension}

From the above discussion, it is clear that only modest deviations from self-similarity in the initial velocity perturbations $w_{0}$ will produce a strongly non-homologous vertical collapse at most realistic $\beta$. We can quantify the magnitude of the initial velocity perturbations $\tilde{\lambda}_{0}$ required to significantly desynchronize one-dimensional collapse by making the approximation (valid for small $\Delta f$ ) that the desynchronization timescale $\Delta t_{1 \mathrm{D}} \approx \Delta f \sqrt{R_{\mathrm{p}}^{3} /\left(G M_{\mathrm{BH}}\right)}$. If we then require $\Delta t_{1 \mathrm{D}}<\tau_{\mathrm{c}}=\chi_{\mathrm{c}} \tau_{*}$, then for a $\gamma=5 / 3$ polytrope (with $\chi_{\mathrm{c}} \approx 8.5$ ) and inwardly directed velocity perturbations we find the condition that

$$
\left|\tilde{\lambda}_{0}\right| \lesssim \frac{\sqrt{2 \beta} \tan \left(2 \chi_{\mathrm{c}} \beta^{-5 / 2}\right)}{1+\sqrt{\beta-1} \tan \left(2 \chi_{\mathrm{c}} \beta^{-5 / 2}\right)} .
$$

The factor of $\approx 2$ inside the argument of the tangent comes from the difference between $\tau_{*}=\sqrt{1 / G \rho_{*}}$ and $\sqrt{R^{3} /\left(G M_{*}\right)}$. This condition grows more restrictive as $\beta$ increases, with the right hand side of Eq. 4.34 roughly proportional to $\beta^{-2}$. 


\section{CHAPTER 4. STRONG COMPRESSION IN TDES}

For one-dimensional stellar collapse, high-resolution hydrodynamical simulations indicate that a highly homologous collapse is physically realized (BL08). As noted before, this is likely due to a combination of two factors: the partial cancellation of stellar pressure with self-gravity, and also that $a_{\mathrm{t}} / a_{\mathrm{g}} \approx\left(R_{\mathrm{t}} / R\right)^{3}$. This explanation is supported by past investigations of stellar tidal disruption in the affine ellipsoids approximation: for example, Fig. 4 in CL83 shows the first order cancellation of pressure and self-gravity for early parts of Phase II. Three results of BL08 further support the validity of the unperturbed free solutions in Phase II of a TDE:

- The actual collapse of the star is visually homologous during Phase II, as seen by the near-linearity of a vertical velocity versus height plot at different times (BL08, Fig. 2). Although the figure deviates slightly from homologous collapse at large radii, possibly due to the fact that the low-density outermost regions of the star are disrupted slightly before the higher density inner regions (like the peeling of onion shells), these outer deviations do not appear to affect the key dynamics of the bounce.

- The maximum central compression $\rho_{\mathrm{c}} \sim \beta^{2 /(\gamma-1)} \rho_{*}$ in accordance with the assumption of fully synchronized tidal free fall (BL08, Eq. 43). We note that this is a geometric proxy for $z_{\min }$.

- The bounce of the collapsing star occurs after pericenter passage (BL08, Table 5). As shown above, this places a strong constraint on the initial velocity perturbations. In particular, let us consider a perfectly homologous collapse for the sake of argument, with $\tilde{w}_{0}=-\tilde{\lambda}_{0} \tilde{z}_{0}$. Eq. 4.32 will only be positive (i.e. bounce after pericenter passage) if $\tilde{\lambda}_{0}<(\sqrt{2} \beta \sqrt{\beta-1})^{-1}$, a rather small perturbation 


$$
(\tilde{\lambda}<0.04 \text { for } \beta=7 \text {, as is relevant here). }
$$

These numerical results indicate that realistic one-dimensional stars behave during Phase II much like the unperturbed free solutions we presented in $\S 3$, supporting our earlier assumption that debris energy "freezes in" from $f=f_{\mathrm{t}}$ down to the bounce, at $f=f_{\mathrm{c}}$.

A final source of one-dimensional desynchronization can arise from the star's internal, pre-disruption density gradient, which will cause the core of the star (with density $\rho_{\text {core }}$ ) to see an effective $\beta_{\text {core }}$ less than the mean $\beta$. If a star on a parabolic orbit has a mean

density $\rho_{*}$, and its core in isolation on that orbit would possess $\beta_{\text {core }}=\beta\left(\rho_{*} / \rho_{\text {core }}\right)^{1 / 3}$, we

can alter our desynchronization formula to read $\tau_{\mathrm{c}} \gtrsim \sqrt{R_{\mathrm{p}}^{3} /\left(G M_{\mathrm{BH}}\right)}\left(f_{\mathrm{c}, \text { core }}-f_{\mathrm{c}}\right)$, or for a $\gamma=5 / 3$ polytrope

$$
\beta_{\text {core }} \gtrsim 1+\left(\tan \left(f_{\mathrm{c}}+8.5 \beta^{-5 / 2}\right)\right)^{-1 / 2} .
$$

This condition is restrictive: if $\beta=3$ (10), it implies a maximum density contrast $\rho_{\text {core }} / \rho_{*}=6$ (53). The fact that this effect does not lead to significantly non-homologous collapse in the BL08 simulations implies that this lower bound on $\beta_{\text {core }}$ must be generous.

\subsubsection{Validity of Free Solutions in Three Dimensions}

The full problem of tidal disruption is three dimensional, and some three dimensional simulations (Laguna et al. 1993; Guillochon et al. 2009) have indicated that onedimensional descriptions of the bounce phase (Luminet \& Carter 1986) may strongly overestimate the degree of compression. However, lack of vertical resolution in the three dimensional simulations makes it difficult to interpret the discrepency, and some high 


\section{CHAPTER 4. STRONG COMPRESSION IN TDES}

resolution simulations (Rosswog et al. 2009) do find degrees of compression closer to our analytic expectations in $\S 4$. Although the impact of higher dimensional effects on Phase III of a TDE will only be resolved through higher resolution hydrodynamical simulations, we present here a simple analytic argument suggesting that a star made of many columns, each undergoing homologous collapse, should attain $z_{\min }$ comparable to one-dimensional predictions.

Three dimensional desynchronization is an important effect that cannot be ignored at high $\beta$ : the bounce timescale $\tau_{\mathrm{c}} \approx 8.5 \tau_{*} \beta^{-4}$ for $\gamma=5 / 3$, while the time it takes the bulk of the star to pass across the tidal radius is $\Delta t_{3 \mathrm{D}} \approx 1.4 \tau_{*}\left(M_{*} / M_{\mathrm{BH}}\right)^{1 / 3}$. This implies that for $\beta$ larger than a critical value,

$$
\beta_{\mathrm{d}}=1.6\left(\frac{M_{\mathrm{BH}}}{M_{*}}\right)^{1 / 12},
$$

$\tau_{\mathrm{c}} \ll \Delta t_{3 \mathrm{D}}$, and the leading edge of the star will collapse and rebound well before the trailing edge. If we assume that the star is truly in tidal free fall during Phase II, and seed velocity perturbations remain as small in three dimensions as has been indicated in one dimensional simulations, then each column of the star will reach its maximum compression at different $t$ but the same $f_{\mathrm{c}}$.

This $f_{\mathrm{c}}$ remains fixed in space, much like a nozzle, as the star passes through it. An example of this "tidal nozzle" has been seen in hydrodynamical simulations of tidal disruptions of white dwarfs; for example, Fig. 6 of Rosswog et al. (2009). Even if we assume the maximum compression predicted by one-dimensional models of stellar collapse $\left(z_{\min } \approx 4.5 R_{*} \beta^{-3}\right.$, for $\left.\gamma=5 / 3\right)$, the sound speed in the stellar midplane, $c_{\mathrm{s}, \mathrm{c}}$, will remain a small fraction of the stellar orbital velocity. Specifically $c_{\mathrm{s}, \mathrm{c}} / V_{\mathrm{p}} \approx \beta^{1 / 2}\left(M_{*} / M_{\mathrm{BH}}\right)^{1 / 3}$, indicating that unphysically large $\beta$ values are required for 


\section{CHAPTER 4. STRONG COMPRESSION IN TDES}

pressure waves from the region of maximum compression to communicate upstream to the Phase II material. Unless three dimensional effects influence earlier stages of a TDE (by seeding large perturbations during the transition from Phase I to Phase II), it seems unlikely that the star will be prevented from reaching the strong compressions suggested by models of one-dimensional collapse. Among other things, this highlights the importance of thermonuclear network calculations for high- $\beta$ TDEs (Luminet \& Pichon 1989).

A further complication is the desynchronization due to the "effective $\beta$ " seen by different regions of the star with different pre-disruption densities. This will have the effect of spreading $f_{\mathrm{c}}$ out over a range of angles for different parts of the star. As the star passes through phase III compression, the nozzle point will move from a starting point slightly ahead of pericenter outward along the orbit (as higher density regions of the star get disrupted), and then inward, back to its starting point. We leave a detailed analysis of this for future work.

With these caveats in mind, we generalize the work of $\S I I I$ to perturbed in-plane free solutions, i.e. where every fluid element at $f=f_{\mathrm{t}}$ has initial positions $\left\{x_{0}, y_{0}\right\}$ but also initial velocities $\left\{u_{0}, v_{0}\right\}$. The in-plane coefficients for the corresponding "perturbed" free solutions are 


$$
\begin{aligned}
A_{\mathrm{p}}= & \frac{1}{\beta^{2}}\left(-8 \tilde{x}_{0} \sqrt{\beta-1}+2 \tilde{y}_{0}\left(\beta^{2}+2 \beta-4\right)\right) \\
& +\frac{2 \sqrt{2}}{\beta^{3 / 2}}\left(\tilde{u}_{0}(2-3 \beta)+\tilde{v}_{0} \sqrt{\beta-1}(\beta-2)\right) \\
B_{\mathrm{p}}= & \frac{1}{5 \beta^{2}}\left(2 \tilde{x}_{0} \sqrt{\beta-1}\left(\beta^{3}-4 \beta^{2}+8\right)+\tilde{y}_{0}\left(9 \beta^{3}\right.\right. \\
& \left.\left.-12 \beta^{2}-8 \beta+16\right)\right)+\frac{1}{5 \sqrt{2} \beta^{3 / 2}}\left(\tilde { u } _ { 0 } \left(\beta^{3}-8 \beta^{2}\right.\right. \\
& \left.+28 \beta-16)+2 \tilde{v}_{0} \sqrt{\beta-1}\left(3 \beta^{2}-6 \beta+8\right)\right) \\
C_{\mathrm{p}}= & \frac{1}{\beta^{2}}\left(\tilde{x}_{0}\left(2 \beta^{2}+\beta-2\right)-2 \tilde{y}_{0} \sqrt{\beta-1}\left(\beta^{2}-1\right)\right. \\
& \left.-\sqrt{2 \beta}\left(\tilde{u}_{0}(1-2 \beta) \sqrt{\beta-1}+\tilde{v}_{0}(\beta-1)^{2}\right)\right) \\
D_{\mathrm{p}}= & -\frac{1}{20 \beta^{2}}\left(\tilde{x}_{0}(\beta-2)+2 \tilde{y}_{0} \sqrt{\beta-1}\right. \\
& \left.-\sqrt{2 \beta}\left(\tilde{u}_{0} \sqrt{\beta-1}+\tilde{v}_{0}\right)\right) .
\end{aligned}
$$

If we now calculate the perturbed specific energy of the free solutions, we find

$$
\begin{aligned}
\epsilon_{p}= & \frac{G M_{\mathrm{BH}} R_{*}}{R_{\mathrm{t}}^{2}}\left(\tilde{x}_{0}(1-2 / \beta)+2 \tilde{y}_{0} \sqrt{\beta^{-1}-\beta^{-2}}\right. \\
& \left.-\sqrt{2 / \beta}\left(\tilde{u}_{0} \sqrt{\beta-1}+\tilde{v}_{0}\right)\right)
\end{aligned}
$$

where the initial velocities have been normalized by $\sqrt{G M_{*} / R_{*}}$. Again, there is no leading order $\beta$ dependence in the specific energy, although a calculation of $\Delta \epsilon_{\mathrm{p}}$ does not find it completely $\beta$-independent as in Eq. 4.21. Nonetheless, the assumption of tidal free fall during Phase II implies clearly that the frozen-in $\Delta \epsilon$ should be, to leading order, independent of $\beta$. As a simple test case, we now apply these perturbed free solutions to a uniformly spinning star, with normalized angular velocity $\tilde{\omega}=\omega / \sqrt{G M_{*} / R_{*}^{3}}$ such that $\tilde{\omega}=1$ is approximately the breakup frequency (and the spin is taken as parallel to the orbital angular momentum). In Eq. 4.41, we relabel $\tilde{x}_{0}=\tilde{r}_{0} \cos \theta_{0}, \tilde{y}_{0}=\tilde{r}_{0} \sin \theta_{0}$, 


\section{CHAPTER 4. STRONG COMPRESSION IN TDES}

$\tilde{u}_{0}=-\tilde{\omega} \tilde{r}_{0} \sin \theta_{0}, \tilde{v}_{0}=\tilde{\omega} \tilde{r}_{0} \cos \theta_{0}$, and then extremize $\Delta \epsilon$ with respect to $\theta_{0}$.

Results for constant $R_{\mathrm{t}}$ are plotted as thin lines in Fig. 4.9; in general, prograde pre-disruption stellar spin will enhance the energy spread $\Delta \epsilon$ by a small factor, $\lesssim 2$. Low $\beta$ and high $\tilde{\omega}$ will maximize this energy spread. Generally, retrograde spins will decrease the energy spread by smaller factors. The difference between prograde and retrograde behavior is geometrical: for prograde spins, the unbound (bound) half of the star tends to receive positive (negative) specific energy perturbations $\Delta \epsilon \sim V_{\mathrm{t}} \omega_{0} r_{0}$, where $V_{\mathrm{t}}$ is the center of mass velocity at the tidal radius. For retrograde spins, this behavior is reversed, although for retrograde spins close to breakup the non-orthogonality of $\hat{X}$ and $\vec{V}$ allows an enhancement to $\Delta \epsilon_{\mathrm{p}}$. The alignment of $\hat{X}$ and $\vec{V}$ at $f=f_{\mathrm{t}}$ increases as $\beta$ increases, so that the highest values of $\beta$ see similar energy spreads for both prograde and retrograde spins.

However, an important complication ${ }^{1}$ is that for spins near breakup (prograde or retrograde), the tidal radius will increase, decreasing the spread in specific debris energy. Using Eq. 13 of Kesden (2012b), we derive that

$$
R_{\mathrm{t}}\left(\tilde{\omega}_{0}\right)=R_{*}\left(M_{\mathrm{BH}} / M_{*}\right)^{1 / 3}\left(1-\tilde{\omega}_{0}^{2}\right)^{-1 / 3} .
$$

This differs by a factor of $2^{1 / 3}$ from the equivalent formula in Kesden (2012b) due to a slight difference in the definition of $R_{\mathrm{t}}$. The effect of the growing tidal radius leads to sharp decreases in $\Delta \epsilon_{\mathrm{p}}$ for spins very close to breakup, and, for $\tilde{\omega}_{0} \gtrsim 0.5$, dominates the dynamical spin effects described in the previous paragraph. The combined effects of a variable tidal radius and the dynamical contribution of stellar spin to frozen-in energy are plotted as thick lines in Fig. 4.9. In general, $\Delta \epsilon_{\mathrm{p}}$ is within a factor of 2 of $\Delta \epsilon_{\mathrm{u}}$ except

\footnotetext{
${ }^{1}$ This was pointed out to us by Michael Kesden.
} 


\section{CHAPTER 4. STRONG COMPRESSION IN TDES}

for $\tilde{\omega}_{0} \gtrsim 0.9$ or $\tilde{\omega}_{0} \lesssim-0.6$.

We note here that large stellar spins misaligned with the orbital angular momentum vector could have a much greater impact on $\Delta \epsilon$ by inducing vertical desynchronization. A thorough investigation of misaligned spin effects is beyond the scope of this work, but as an idealized limiting case we apply Eq. 4.34 to approximate, as a function of $\beta$, the maximum stellar spin allowed before the Phase III bounce would be vertically desynchronized. Specifically, we set $w_{0}=\omega_{0} r_{0}$. More specifically, Eq. 4.34 lets us calculate the regions of parameter space in which the dynamical desynchronization of stellar compression (due to the spread of velocities in a spinning star) stretches out for longer than the hydrodynamical bounce time. We plot these results in Fig. 4.10, and find that combinations of high $\beta$ and relatively rapid values of stellar spin are needed to strongly desynchronize the vertical collapse.

Fig. 4.9 can be taken as representative of the effects of both primordial stellar spin, and the angular momentum imparted during tidal spin-up of the star prior to its full disruption, during the transition between phases I and II of a TDE. Tidal spin-up is unlikely to produce misaligned spin, however, so the more dramatic type of desynchronization suggested by Fig. 4.10 can only come from the star's original, pre-disruption spin. Furthermore, tidal spin-up may produce effects similar to the thin curves of Fig. 4.9, provided the spin-up occurs close to the tidal radius of an equivalent, nonspinning star.

For one dimensional stellar collapse, the frozen-in $\Delta \epsilon$ will dominate the post-bounce $\Delta \epsilon$ for all $\beta$. For three dimensional collapse, the numerical literature is less clear, but we have argued here that three-dimensional effects are unlikely to strongly redistribute 
CHAPTER 4. STRONG COMPRESSION IN TDES

energy to in-plane motion, with the possible exception of when sufficiently rapid stellar spin is misaligned with the orbital plane, or perhaps when a similar misalignment between SMBH spin and orbital angular momentum exists.

\subsection{General Relativistic Corrections}

The results of all prior sections have assumed purely Newtonian gravity; however, for SMBHs with masses $M_{\mathrm{BH}}>10^{6} M_{\odot}$, tidal disruption of solar-type stars occurs at an orbital distance $R_{\mathrm{t}} \lesssim 50 R_{\mathrm{g}}$. Here the gravitational radius $R_{\mathrm{g}}=G M_{\mathrm{BH}} / c^{2}$. At these small separations, ballistic motion follows the geodesics of the Schwarzschild or Kerr metric rather than free-fall trajectories in Newtonian gravity: general relativity (GR) is important. A fully relativistic analysis of the problem of tidal disruption is beyond the scope of this paper, although it has been treated in the past in the case of the affine model (Luminet \& Marck 1985), and in one-dimensional hydrodynamical simulations (Brassart \& Luminet 2010). If we treat the internal dynamics of the star in a Newtonian way (i.e. assume tangentially flat space-time in the small region occupied by the star), then Eq. 4.12's description of vertical collapse will be modified, to become

$$
\ddot{z}_{\mathrm{GR}}=\frac{\partial \Psi}{\partial z}\left(1+3 \frac{L^{2}}{r^{2}}\right),
$$

where $L$ is the orbital angular momentum of the star, $r$ is the orbital radius of the star (both in geometrized units) and we have limited ourselves to non-spinning black holes. The qualitative results of both Luminet \& Marck (1985) and Brassart \& Luminet (2010) were that the increased strength of the GR tidal field (relative to Newtonian gravity) can actually result in multiple vertical collapses, each followed by separate bounces which are

reversed by the relativistically enhanced tidal field. For all but the most deeply-plunging 


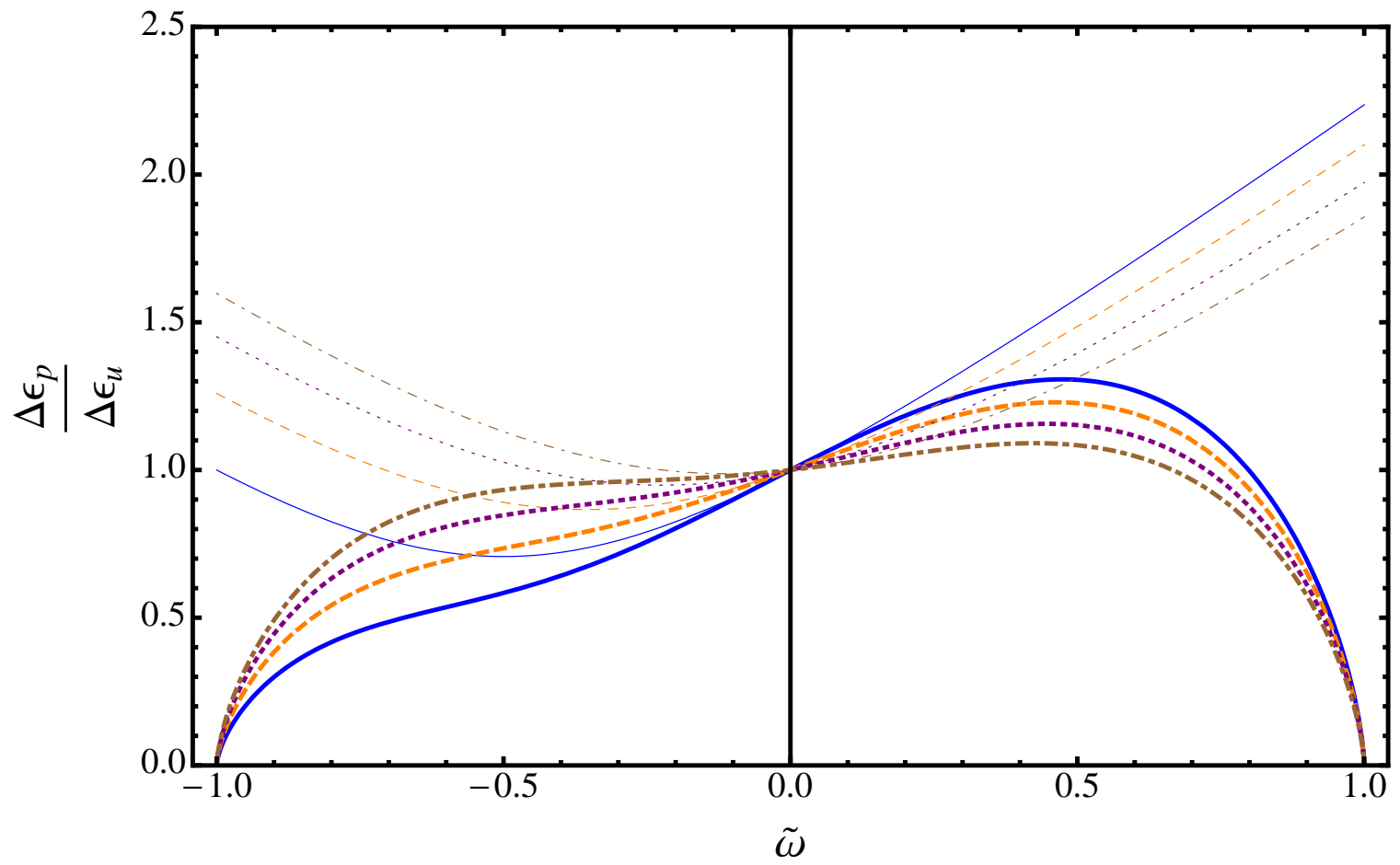

Figure 4.9.- : The enhancement $\Delta \epsilon_{\mathrm{p}} / \Delta \epsilon_{\mathrm{u}}$ to the energy spread for initially unperturbed free solutions, when pre-disruption stellar spin (along an axis parallel to orbital angular momentum) is considered. The energy spread is plotted against pre-disruption spin $\tilde{\omega}$ , where $\tilde{\omega}$ is stellar spin normalized by the breakup spin $\sqrt{G M_{*} / R_{*}^{3}}$. As in previous plots, the solid blue, dashed orange, dotted purple, and dot-dashed cyan curves represent $\beta=2, \beta=4, \beta=10$, and $\beta=40$, respectively. The thin lines represent the dynamical effects of stellar spin when the tidal radius is held constant; in the more realistic thick lines, the tidal radius is also allowed to vary with stellar spin. 
CHAPTER 4. STRONG COMPRESSION IN TDES

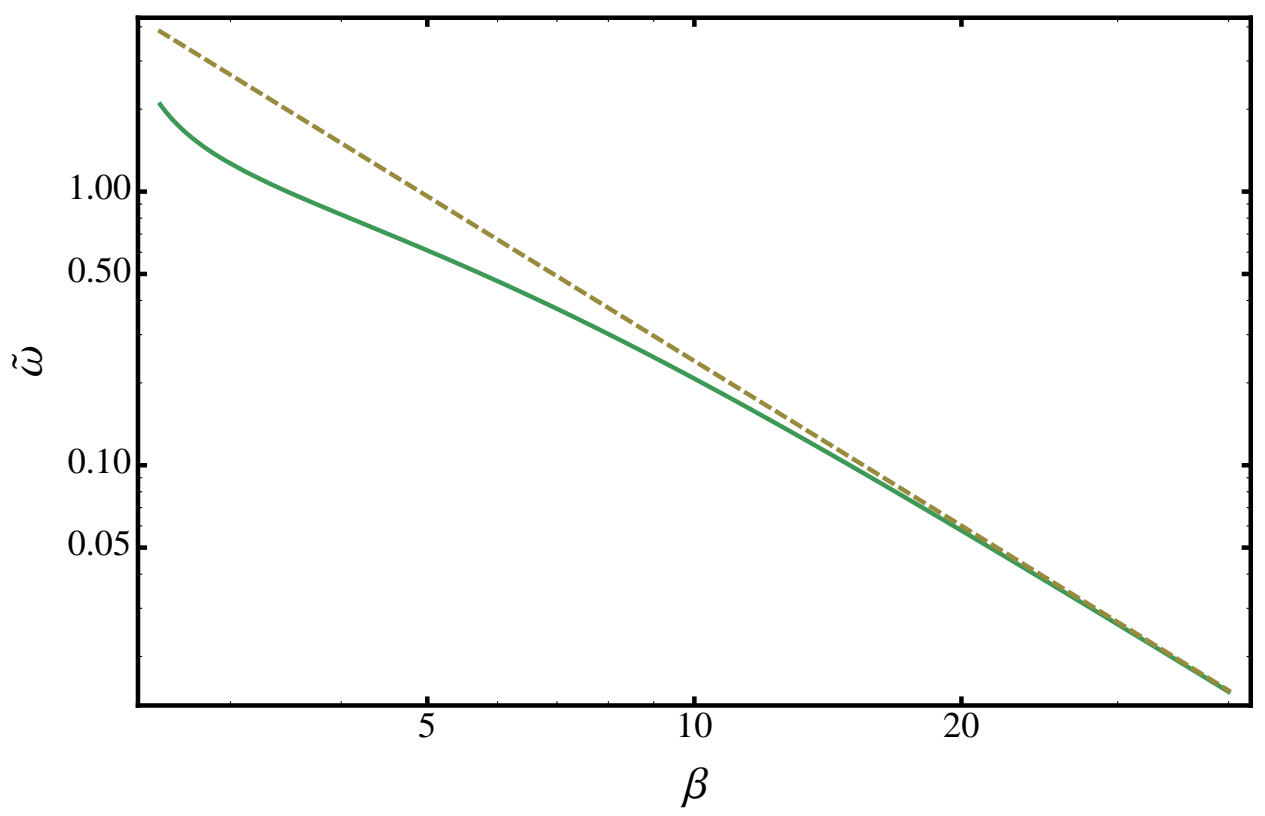

Figure 4.10.- : The maximum value of normalized stellar spin $\tilde{\omega}$ that will not produce significant 1D desynchronization leading into the phase III bounce. We plot the exact value calculated from Eq. 4.34 as a solid green line, and the asymptotic behavior $\tilde{\omega} \lesssim 2 \sqrt{2} \chi_{\mathrm{c}} \beta^{-2}$ as a dashed yellow line. Regions above the curves will experience desynchronization of vertical collapse. 


\section{CHAPTER 4. STRONG COMPRESSION IN TDES}

TDEs $(\beta \gtrsim 30)$, the maximum compression is obtained on the first vertical collapse and is similar to the Newtonian $z_{\min }$ (Luminet \& Marck 1985, Fig. 10). Therefore, even though the formation of multiple outgoing shockwaves could be an important outcome of relativistic compression, the first-order spread in debris energy is unlikely to be affected by multiple compressions for $\beta \lesssim 30$.

A separate relativistic effect concerns modifications to the pre-bounce spread in debris energy, $\Delta \epsilon$. Eq. 4.3 was derived by Taylor expanding the Newtonian gravitational potential about the star's position when it crossed into the tidal sphere, then subtracting the zeroth-order component. We will now repeat that procedure for a post-Newtonian (PN) effective potential which incorporates leading-order GR effects for non-spinning, Schwarzschild black holes. Specifically, we use the 1PN harmonic coordinate Lagrangian presented in Blanchet (2006, Eq. 174):

$$
\begin{aligned}
& \mathcal{L}^{\text {harm }}=\frac{G m_{1} m_{2}}{2 r_{12}}+\frac{m_{1} v_{1}^{2}}{2}+\frac{1}{c^{2}}\left(-\frac{G^{2} m_{1}^{2} M_{2}}{2 r_{12}^{2}}+\frac{m_{1} v_{1}^{4}}{8}\right. \\
& \left.+\frac{G m_{1} m_{2}}{r_{12}}\left(-\frac{1}{4}\left(\vec{n}_{12} \cdot \vec{v}_{1}\right)\left(\vec{n}_{12} \cdot \vec{v}_{2}\right)+\frac{3}{2} \vec{v}_{1}^{2}-\frac{7}{4}\left(\vec{v}_{1} \cdot \vec{v}_{2}\right)\right)\right)
\end{aligned}
$$

We define the effective potential as $\Phi_{\text {eff }}=K-\mathcal{L}^{\text {harm }}$, where $K$ represents the kinetic energy component of the Lagrangian, i.e. those terms which depend only on velocities. This equation was derived for arbitrary mass-ratio systems, but here we identify the star as particle 1, the SMBH as particle 2, and have dropped all terms proportional to $v_{2}$ or $m_{1} / m_{2}$. The Taylor expansion of $\Phi_{e f f}$ up to first PN order, around $R=R_{\mathrm{t}}$, is given by

$$
\Delta \epsilon_{\mathrm{GR}}=\frac{G M_{\mathrm{BH}} R_{*}}{R_{\mathrm{t}}^{2}}\left(1+\frac{3 V_{\mathrm{t}}^{2}}{2 c^{2}}-\frac{R_{\mathrm{g}}}{R_{\mathrm{t}}}\right) .
$$

From this equation, it is clear that GR corrections to the Newtonian potential will only matter for large, $M_{\mathrm{BH}}>10^{7} M_{\odot}$ SMBHs, with tidal radii close to or within 


\section{CHAPTER 4. STRONG COMPRESSION IN TDES}

the ISCO. However, all TDEs due to such black holes, or even more massive ones (Kesden 2012b), will have debris energy spreads modified by GR around the $\sim 2$ level (although we caution that our PN approximation breaks down for tidal radii approaching the ISCO). In this discussion we have neglected spin effects, but they may also play an important role for the subset of TDEs with $R_{\mathrm{p}} \lesssim R_{\mathrm{ISCO}}$. During completion of this paper, a more precise formalism was presented for estimating the GR corrections described in this section (Kesden 2012a). The results indicate generally small (factors $\lesssim 3$ GR corrections which are maximized when the spread in energy of a spherical star is calculated near the ISCO, in qualitative agreement with our findings. We note however that Kesden (2012a) uses the older, less accurate approach to treating "frozen-in" debris energy, i.e. evaluating the spread in energy at $R_{\mathrm{p}}$ rather than $R_{\mathrm{t}}$. The primary difference between our approaches is that if debris energy freezes in at the tidal radius, GR corrections to $\Delta \epsilon$ can only reach large, $\sim 2$ levels for $M_{\mathrm{BH}} \gtrsim 10^{7.5} M_{\odot}$. TDEs around low-mass SMBHs will see negligible GR corrections to $\Delta \epsilon$, even if $\beta$ is large.

\subsection{Gravitational Waves}

The parabolic motion of a star past orbital pericenter will produce low-frequency gravitational waves due to time variation in the quadrupole moment of the star-SMBH system. For pericenters $R_{\mathrm{p}}<R_{\mathrm{t}}$, past work has indicated that such a signal could be marginally detectable with a LISA-like instrument (Kobayashi et al. 2004). Analogous work has focused on the inspiral of a white dwarf (WD) into an intermediate-mass black hole, where a similar signal could be generated by a violent disruption (Rosswog et al. 2009; Haas et al. 2012), or a longer-lived GW signal could be accompanied by 


\section{CHAPTER 4. STRONG COMPRESSION IN TDES}

electromagnetic transients due to inspiral and stable mass transfer (Zalamea et al. 2010). An alternate, internal source of GWs in TDEs comes from time variation of the star's own quadrupole moment during the Phase III vertical rebound, which was estimated in the past to generate gravitational waves with strain $h \propto \beta^{3}$ (Guillochon et al. 2009).

In this section, we present more detailed estimates of the "internal" GWs due to stellar pancaking and rebound, a process analogous to GW generation during core-collapse supernovae (CCSNe). As we shall demonstrate, TDE GWs are weakened relative to those in CCSNe due to lower collapse velocities and bounce accelerations, but are increased due to the large degree of stellar asymmetry, and perhaps also by the correspondingly long lever arm of collapse in the quadrupole moment tensor.

Specifically, we consider GW emission at the moment of maximum stellar compression, which we for now take to be synchronized throughout the star (but which will actually occur at different times for each point in the star, as seen in §5). The two polarization components of a GW signal, $h_{+}$and $h_{\times}$, can be read off of the transverse traceless GW strain

$$
h_{\mathrm{ij}}^{\mathrm{TT}}=\frac{2 G}{d c^{4}} \ddot{\mathrm{J}}_{\mathrm{ij}}^{\mathrm{TT}},
$$

where $d$ is the distance from the observer to the source, $J_{i j}=I_{i j}-\frac{1}{3} \delta_{i j} \delta^{k l} I_{k l}$ is the reduced quadrupole moment tensor, $J_{i j}^{\mathrm{TT}}$ is a projection of $J_{i j}$, and $I_{k l}$ is the standard 
CHAPTER 4. STRONG COMPRESSION IN TDES

quadrupole moment tensor:

$$
\begin{aligned}
& \ddot{I}_{k l}=\int \mathrm{d}^{3} \vec{r} \rho \times \\
& \left(\begin{array}{ccc}
2 \dot{x}^{2}+2 x \ddot{x} & 2 \dot{x} \dot{y}+\ddot{x} y+x \ddot{y} & 2 \dot{x} \dot{z}+\ddot{x} z+x \ddot{z} \\
2 \dot{x} \dot{y}+\ddot{x} y+x \ddot{y} & 2 \dot{y}^{2}+2 y \ddot{y} & 2 \dot{y} \dot{z}+\ddot{y} z+y \ddot{z} \\
2 \dot{x} \dot{z}+\ddot{x} z+x \ddot{z} & 2 \dot{y} \dot{z}+\ddot{y} z+y \ddot{z} & 2 \dot{z}^{2}+2 z \ddot{z}
\end{array}\right) .
\end{aligned}
$$

To order of magnitude in the limit of fully synchronous vertical collapse, and neglecting the (weak) $\beta$ dependence of all $x$ and $y$ terms, we then have

$$
\frac{\ddot{I}_{k l}}{M_{*} R_{*}^{2} \tau_{*}^{-2}} \sim \int \mathrm{d}^{3} \vec{r}\left(\begin{array}{ccc}
\beta^{0} & \beta^{0} & \beta^{5} \\
\beta^{0} & \beta^{0} & \beta^{5} \\
\beta^{5} & \beta^{5} & \beta^{2}
\end{array}\right),
$$

where we have taken $\gamma=5 / 3$ (as we will for the remainder of this section) and approximated $z \sim \beta^{-3} R_{*}, \dot{z} \sim \beta \sqrt{G M_{*} / R_{*}}$, and $\ddot{z} \sim \dot{z} / \tau_{\mathrm{c}} \sim \beta^{5} G M_{*} / R_{*}^{2}$.

If only diagonal terms are considered, the second derivative of the quadrupole tensor will be $\propto \beta^{2}$. However, the extremely steep $\beta^{5}$ dependence of the off-diagonal $\ddot{I}_{\mathrm{xz}}$ and $\ddot{I}_{\mathrm{yz}}$ terms indicates that viewing angles not closely aligned with $\hat{z}$ could in principle observe copious GW production. This steeper $\beta$ dependence arises from the $y \ddot{z}$ and $x \ddot{z}$ terms, which couple the long (and only weakly $\beta$ dependent) lever arms within the orbital plane to the rapid vertical acceleration of the bounce. We note that physically, $\ddot{I}_{\mathrm{xz}} \sim 10 \ddot{I}_{\mathrm{yz}}$ because for the large $\beta$ where GW emission is relevant, $\hat{y}$ will be aligned with the star's longest principal axis.

However, two degrees of symmetry present in this problem will substantially reduce the magnitudes of $\ddot{I}_{x z}$ and $\ddot{I}_{y z}$. The free solutions indicate that to lowest order, tidally free-falling bodies should possess reflection symmetry about their in-plane principal 


\section{CHAPTER 4. STRONG COMPRESSION IN TDES}

axes. There is also an additional symmetry of reflection about the orbital plane. If these symmetries are exact, the off-diagonal terms in Eq. 4.47 will integrate to 0. The in-plane symmetries are broken when $R_{*} / R \sim 1$, i.e. for deeply plunging disruptions around low-mass SMBHs. The orbital plane reflection symmetry is more robust, and likely can only be broken by misalignment between the orbital plane and SMBH or stellar spin, which is beyond the scope of this paper. For the remainder of this section, we treat GW emission from off-diagonal terms as speculative, but the large magnitude of these terms in the integrand should motivate future work on disruptions of spinning stars, or TDES around spinning SMBHs.

For $\beta<\beta_{\mathrm{d}}$, three-dimensional desynchronization is unimportant and the star collapses almost simultaneously, emitting GWs with a peak frequency of $\approx 1 / \tau_{\mathrm{c}}$, which, using the calibration of $\tau_{\mathrm{c}} \approx 8.5 \beta^{-4} \tau_{*}$ from the affine model and one-dimensional hydro simulations $(\S 4)$, gives

$$
f_{\mathrm{GW}} \approx 15 \mathrm{~Hz}\left(\frac{\beta}{25}\right)^{4} m_{*}^{1 / 2} r_{*}^{-3 / 2},
$$

where we have normalized $m_{*}=M_{*} / M_{\odot}$ and $r_{*}=R_{*} / R_{\odot}$. Low mass stars have an easier time achieving high frequencies; if we use the relation $R_{*} \propto M_{*}^{0.8}$ for main sequence stars with $M_{*} \leq M_{\odot}$, we find $f_{\mathrm{GW}} \approx 10 \mathrm{~Hz}$ at $\beta=15$, for $M_{*}=0.1 M_{\odot}$.

These frequencies are located on the far edge of the Advanced LIGO band, although with steep $\beta$ dependence. Because the three-dimensional desynchronization discussed in $\S 5$ results in the leading edge of the star collapsing before the trailing edge (which lags by a time $\Delta t_{3 \mathrm{D}}$ ), the GW signal will be smeared out over a range of frequencies between $1 / \Delta t_{3 \mathrm{D}}$ and $1 / \tau_{\mathrm{c}}$ when $\beta>\beta_{\mathrm{d}}$. This smears out the gravitational wave emission by a 
CHAPTER 4. STRONG COMPRESSION IN TDES

factor $\Delta t_{3 \mathrm{D}} / \tau_{\mathrm{c}}$, giving us the strain estimates

$$
\begin{aligned}
h_{+} \approx 1 \times 10^{-25} m_{*}^{2} r_{*}^{-1} d_{10}^{-1} \begin{cases}\left(\frac{\beta}{\beta_{\mathrm{d}}}\right)^{2}, & \beta \lesssim \beta_{\mathrm{d}} \\
\left(\frac{\beta}{\beta_{\mathrm{d}}}\right)^{-2} M_{6}^{1 / 6}, & \beta \gtrsim \beta_{\mathrm{d}}\end{cases} \\
h_{\times} \approx 1 \times 10^{-23} \Xi m_{*}^{2} r_{*}^{-1} d_{10}^{-1} \begin{cases}\left(\frac{\beta}{\beta_{\mathrm{d}}}\right)^{5}, & \beta \lesssim \beta_{\mathrm{d}} \\
\left(\frac{\beta}{\beta_{\mathrm{d}}}\right) M_{6}^{5 / 12}, & \beta \gtrsim \beta_{\mathrm{d}}\end{cases}
\end{aligned}
$$

Here $d_{10}$ is distance to the source normalized to 10 megaparsecs, and for clarity (i.e. to separate diagonal and off-diagonal components of $\ddot{J}_{i j}$ into different polarization states) we have assumed a line of sight along the $y$ axis so that $d h_{+}=G\left(\ddot{J}_{x x}-\ddot{J}_{z z}\right) / c^{4}$, and $d h_{\times}=2 G \ddot{J}_{x z} / c^{4}$. As in $\S 5, \beta_{\mathrm{d}} \approx 6$ is the critical $\beta$ value above which TDEs experience significant three dimensional desynchronization. We have defined a parameter, $\Xi$ (which is most likely $\ll 1$ ), to parametrize the unknown degree of reflection asymmetry in Phase III of a TDE.

The prospects for high frequency GW observation of TDEs involving main sequence stars appear dim, unless $\Xi \gtrsim 0.1$. If we limit ourselves to GWs from $\ddot{I}_{z z}$, then disruptions of abundant low mass stars have an easier time falling within the Advanced LIGO band, but produce too little strain to be detected; disruptions of solar-type stars produce a barely detectable strain at $d \sim 1 \mathrm{Mpc}$, but will lie outside the Advanced LIGO band for $\beta \lesssim 25$

Tidal disruptions of white dwarfs by intermediate mass black holes appear more promising: a WD of mass $1 M_{\odot}$ and radius $6 \times 10^{6} \mathrm{~m}$ disrupted at $\beta=5$ by a $10^{4} M_{\odot}$ IMBH will produce $h_{+} \approx 6 \times 10^{-24}$ from a distance of $20 \mathrm{Mpc}$ (neglecting all strain from off-diagonal terms in $\ddot{J}_{i j}$ ). The peak emission frequency $f_{\mathrm{GW}} \approx 60 \mathrm{~Hz}$, but the existence 


\section{CHAPTER 4. STRONG COMPRESSION IN TDES}

of IMBHs is sufficiently uncertain that we do not attempt a rate estimate. We note that the level of emission seen at $\sim 50 \mathrm{~Hz}$ frequencies in full numerical relativity simulations of WD-IMBH disruptions (Haas et al. 2012) was approximately $\times 10^{3}$ smaller than our prediction. This may not surprising, as the maximum density enhancement seen in these simulations is $\lesssim 10$, not $\sim 200$ as predicted by one-dimensional models $(\gamma=5 / 3, \beta \approx 10)$. It is likely that this discrepency is at least partially due to insufficient vertical resolution: after disruption, the smallest grid cell in these simulations is $\approx R_{\mathrm{WD}} / 40$. Furthermore, Newtonian and pseudo-Newtonian simulations of $\beta \approx 10$ WD TDEs (Rosswog et al. 2009) find maximum degrees of vertical compression $\sim 100$, in approximate agreement with the arguments presented in $\S 4$ and 5.

\subsection{Observational Implications}

In this section, we discuss the observational implications of a revised $\Delta \epsilon$ for optical transient searches. In general, we predict longer decay times but lower initial mass fallback rates than prior works (Strubbe \& Quataert 2009).

Using the introduced index $n$, we rederive here a number of important consequences of Eq. 4.4 in a parametrized way. The fallback time for the most tightly bound debris is

$$
t_{\text {fall }}=3.5 \times 10^{6} \sec k^{-3 / 2} \beta^{-3 n / 2} M_{6}^{1 / 2} m_{*}^{-1} r_{*}^{3 / 2},
$$

where we have used the normalizations $M_{6}=M_{\mathrm{BH}} /\left(10^{6} M_{\odot}\right), m_{*}=M_{*} / M_{\odot}$, and $r_{*}=R_{*} / R_{\odot}$. The rate of mass fallback evolves with time $t$ as

$$
\dot{M}_{\text {fall }} \approx \frac{M_{*}}{3 t_{\text {fall }}}\left(\frac{t}{t_{\text {fall }}}\right)^{-5 / 3},
$$

and is initially super-Eddington for disruptions of solar-type stars by SMBHs with 


\section{CHAPTER 4. STRONG COMPRESSION IN TDES}

$M_{\mathrm{BH}} \lesssim 10^{7.5} M_{\odot}$. Assuming a radiative efficiency $0<\eta<1$, the peak (i.e. time of first pericenter return) mass fallback rate is given by

$$
\frac{\dot{M}_{\text {peak }}}{\dot{M}_{\text {Edd }}} \approx 133 \eta_{-1} k^{3 / 2} \beta^{3 n / 2} M_{6}^{-3 / 2} m_{*}^{2} r_{*}^{-3 / 2},
$$

implying that the maximum black hole mass that can undergo a phase of super-Eddington accretion is given by

$$
M_{\mathrm{BH}, \max }=2.6 \times 10^{7} M_{\odot} \eta_{-1}^{2 / 3} k \beta^{n} m_{*}^{4 / 3} r_{*}^{-1}
$$

The mass fallback rate becomes sub-Eddington at a time

$$
t_{\mathrm{Edd}}=6.6 \times 10^{7} \sec \eta_{-1}^{3 / 5} k^{-3 / 5} \beta^{-3 n / 5} M_{6}^{-2 / 5} m_{*}^{1 / 5} r_{*}^{3 / 5}
$$

although if $t_{\text {Edd }}<t_{\text {fall }}$ there is no super-Eddington accretion phase. Here we have set $\eta_{-1}=\eta / .1$. Considering the dependences of Eqs. 4.51, 4.53, 4.54, and 4.55 on $n$ we see that adopting Eq. 4.3 can have dramatic effects on TDEs from stars on deeply plunging $(\beta>3)$ orbits. In particular, using the correct values of $\Delta \epsilon$ reduces the peak mass fallback rate, decreasing the maximum SMBH mass that can produce a super-Eddington accretion phase. On the other hand, for TDEs with super-Eddington accretion, the duration of the super-Eddington phase is extended for (realistic) low- $n$ values.

We plot the effect of $n$ on the mass fallback rate in Fig. 4.11. In previous literature $(n=2)$ a wide variety of mass fallback curves were possible, with high peaks and fast decay times accompanying large $\beta$ values. If $n=0$, however, the mass fallback rate is generally independent of $\beta$. Under simplifying assumptions about the relationship of disk luminosity to $\dot{M}$ (often but inaccurately taken as $L \propto \dot{M}$; for complications see Lodato et al. (2009); Lodato \& Rossi (2011)), the fallback timescale can be inferred by sufficiently long lightcurve observations. Alternatively, in the future it may be possible to measure $t_{\text {fall }}$ directly, by measuring the delay between the onset of accretion and a 


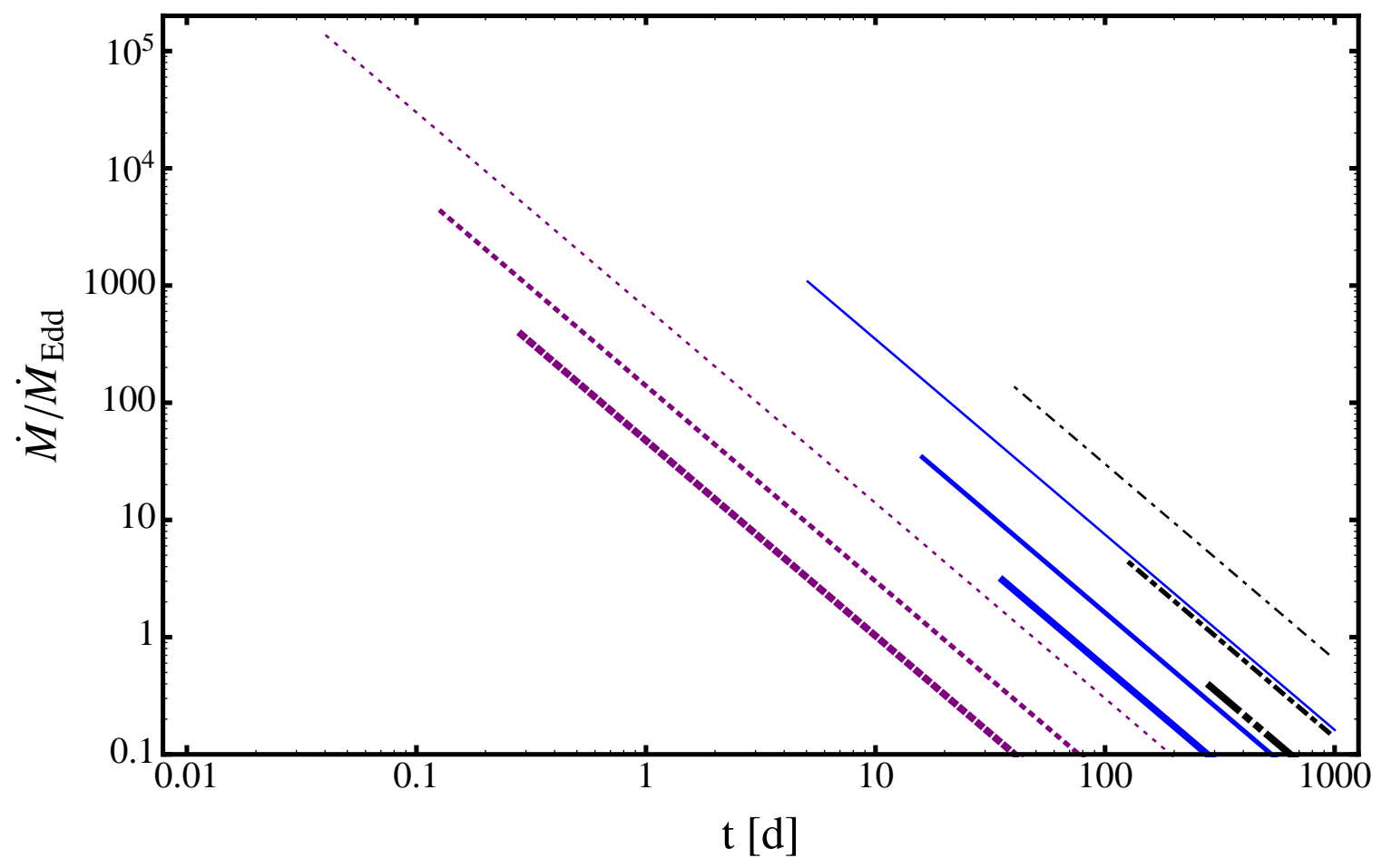

Figure 4.11.- : Mass fallback curves (normalized by the Eddington fallback rate) versus time since disruption in days, for a variety of TDEs. SMBH masses of $10^{6} M_{\odot}, 10^{7} M_{\odot}$, and $5 \times 10^{7} M_{\odot}$ are plotted as thin, normal, and thick curves, respectively. Likewise, $\beta$ values of 1,2, and 10 are plotted as black dot-dot-dashed, blue solid, and purple dotted curves assuming $n=2$, the power law index defined by $\Delta \epsilon \propto \beta^{n}$ - see Eq. 4.4. If $n=0$, the black dot-dot-dashed curves represent all $\beta$ values. Here we consider solar-type stars, and for simplicity set $k=1$ and $\eta=0.1$. 


\section{CHAPTER 4. STRONG COMPRESSION IN TDES}

prompt signal accompanying stellar disruption (either X-ray shock breakout or GWs). In either case, the $\beta$ independence of $t_{\text {fall }}$ will simplify parameter extraction, in particular measurement of $M_{\mathrm{BH}}$.

Adopting $n=0$ will also alter the distribution of $\beta$ in the TDEs detected by individual wide-field surveys, $\dot{N}_{\text {det }}(\beta)$. This is a quantity distinct from the distribution of the intrinsic TDE rate, $\dot{N}_{\mathrm{TDE}}(\beta)$, which scales as $\dot{N}_{\mathrm{TDE}} \propto \beta^{-1}$ for two-body relaxation in the "pinhole" regime (Brassart \& Luminet 2008). Alternatively, if the dominant source of loss cone fueling is two-body relaxation in the "diffusion" regime, almost all TDEs will have $\beta=1$; however, since most SMBHs are supplied with stars coming from the boundary between these regimes we will consider the pinhole regime for the remainder of this section (as it is the relaxational regime with nontrivial $\beta$ dependence). If the TDE rate is dominated by the effects of an axisymmetric (Vasiliev \& Merritt 2013) or triaxial (Merritt \& Poon 2004) stellar potential, the distribution of $\beta$ will also follow the pinhole regime's scaling.

If we first consider UV or soft X-ray surveys sensitive to the peak frequencies of TDE disk emission, then the peak luminosity $L \propto \dot{M}_{\text {peak }} \propto \beta^{3 n / 2}$, implying a survey horizon $r_{\text {hor }} \propto \beta^{3 n / 4}$ and a detection rate $\dot{N}_{\text {det }} \propto \beta^{(9 n-4) / 4}$. Optical detections of TDE disks will not be sensitive to the event's bolometric luminosity but rather to emission on the Rayleigh-Jeans tail, for which $L \propto \dot{M}^{1 / 4}$ and $\dot{N}_{\text {det }} \propto \beta^{(9 n-16) / 16}$ (Lodato \& Rossi 2011). However, both of these scaling relations for $\dot{N}_{\text {det }}$ assume a purely flux-limited survey; in the old picture of TDE energy spread $(n=2)$, high- $\beta$ events would be favored by their high flux but disfavored by their shorter timescales of peak emission. If the timescale of peak emission, $t_{\text {fall }}$, is less than the survey cadence, $t_{\text {cad }}$, then the probability of detection will be approximately reduced by the factor $t_{\text {fall }} / t_{\text {cad }}$. This gives $\dot{N}_{\text {det }} \propto \beta^{(3 n-4) / 4}$ and 


\section{CHAPTER 4. STRONG COMPRESSION IN TDES}

$\dot{N}_{\text {det }} \propto \beta^{(-15 n-16) / 16}$ for X-ray and optical disk emission, respectively.

Although the details remain uncertain, several recent papers (Strubbe \& Quataert 2009; Lodato \& Rossi 2011) have predicted that super-Eddington, radiation-driven outflows may dominate early emission from TDE accretion disks, particularly at long wavelengths. Using a simple blackbody model with peak frequency $\nu_{\mathrm{bb}}$ (Lodato \& Rossi 2011), which predicts a peak luminosity $L \propto \beta^{97 / 24}$ at frequencies $\nu<\nu_{\mathrm{bb}}$, or $L \propto \beta^{2 / 5}$ for $\nu>\nu_{\mathrm{bb}}$, we can repeat the above calculations. The $\beta$ dependence of all different scenarios are presented in Table 1. With $n=0$, all observational strategies are biased in favor of low $\beta$ detections, including many strategies that once favored high $\beta$ TDEs.

\subsection{Discussion}

In this paper, we have analyzed the tidal disruption and free fall of a star in the context of "free solutions" to the Hill equations of the parabolic restricted three-body problem. The important conclusions of this work are the following:

1. During the tidal disruption of a star, debris energy freezes in at $R=R_{\mathrm{t}}$, not $R=R_{\mathrm{p}}$.

2. Consequently, the spread in debris energy is smaller than in past analytic predictions. This will result in flares with longer fallback times, i.e. ones that decay more slowly but have smaller initial fallback rates. Fewer TDEs will drive powerful super-Eddington outflows than has been predicted in the past.

3. The spread in debris energy is generally dominated by the freeze-in energy, although redistribution of the kinetic energy of vertical collapse to in-plane motions 


\begin{tabular}{r||r|r|r} 
Scenario & $n=2\left(\frac{t_{\text {fall }}}{t_{\text {cad }}}>1\right)$ & $n=2\left(\frac{t_{\text {fall }}}{t_{\text {cad }}}<1\right)$ & $n=0$ \\
\hline Disk, $\nu \approx \nu_{\mathrm{bb}}$ & $7 / 2$ & $1 / 2$ & -1 \\
Disk, $\nu<\nu_{\mathrm{bb}}$ & $1 / 8$ & $-23 / 8$ & -1 \\
$\mathrm{SE}, \nu \approx \nu_{\mathrm{bb}}$ & $-2 / 5$ & $-17 / 5$ & $-7 / 10$ \\
$\mathrm{SE}, \nu<\nu_{\mathrm{bb}}$ & $81 / 16$ & $33 / 16$ & $-33 / 16$
\end{tabular}

Table 4.1:: Here we display the scaling exponents $s$ for the $\beta$ dependence of the TDE detection rate, $\dot{N}_{\text {det }}(\beta) \propto \beta^{s}$. In the first column we describe the frequency and source of emission (SE indicates a super-Eddington outflow); in the second we give $s$ for the standard scenario $n=2$ and a high-cadence survey; in the third we again consider $s$ in the standard scenario, but for a slow-cadence survey; in the final column we give $s$ for our revised $\Delta \epsilon$, with $n=0$, at any cadence. In this table $\nu_{\mathrm{bb}}$ refers to the "blackbody" frequency of peak emission (the super-Eddington outflow is assumed to have a thermal spectrum; the disk emission is better modeled as a multicolor blackbody). 


\section{CHAPTER 4. STRONG COMPRESSION IN TDES}

may result in slight variation in $\Delta \epsilon$ for low $\beta(\lesssim 5)$. Rapidly spinning stars may see a stronger version of this effect at high $\beta$ if their spins are misaligned with the orbital angular momentum vector.

4. The leading order GR corrections to the frozen-in value of $\Delta \epsilon$ are small, and generally negligible unless $R_{\mathrm{p}} \lesssim 6 R_{\mathrm{g}}$. For such TDEs (i.e. all TDEs for SMBH masses above $10^{7.5} M_{\odot}$ ) we have derived for the first time the $1 \mathrm{PN}$ modifications to $\Delta \epsilon$.

5. The free solution model we have introduced is, in the limit of spherical and static initial conditions, an approximate simplification of the affine model . However, the ability to include a range of nonspherical or dynamic initial conditions gives it a degree of flexibility not present in the affine model. Furthermore, the deformations to an initially spherical body in tidal free fall are not ellipsoids, as is assumed by the affine model.

6. Gravitational waves are generated from variation in the internal quadrupole moment of a tidally disrupting star, reaching peak amplitude at the moment of maximum compression and bounce. GW emission is likely dominated by a single term in the quadrupole moment tensor, $\ddot{I}_{z z} \propto \beta^{2}$. For main sequence stars, these are unlikely to be detectable by ground-based GW interferometers. GWs from the tidal disruption of WDs by IMBHs are more promising targets, and for modest $\beta$ values $(\sim 5)$ could be detectable to tens of megaparsecs.

7. Gravitational wave emission from nondiagonal terms in the star's quadrupole moment could alter the previous conclusion, since $\ddot{I}_{x z}, \ddot{I}_{y z} \propto \beta^{5}$. However, in order for these terms to possess a nonzero prefactor, the reflection symmetry of the TDE 


\section{CHAPTER 4. STRONG COMPRESSION IN TDES}

about the orbital plane must be broken by either SMBH or stellar spin. Whether this can be done without desynchronizing the vertical collapse and weakening the $\beta$ dependence of $\ddot{I}_{x z}, \ddot{I}_{y z}$ is unclear.

The existing hydrodynamical literature did not until very recently support the first three of these conclusions. With a few exceptions, prior work has focused mainly on the common $\beta=1$ events, for which Eqs. 4.2 and 4.3 are identical. In Laguna et al. (1993), the authors conducted SPH simulations in a static Schwarzschild background geometry for $\beta=\{1,5,10\}$. They found that the fallback time scaled approximately as $t_{\text {fall }} \propto \beta^{-1.5}$ and the velocity of unbound ejecta $v_{\mathrm{ej}} \propto \beta^{0.5}$, both of which imply the intermediate value of $n=1$, for the power-law index defined in Eq. 4.4 as $\Delta \epsilon \propto \beta^{n}$. However, the limited resolution of their simulations (7000 SPH particles) makes it unclear whether they would have possessed the midplane resolution to resolve the phase of maximum compression;

indeed, they find that $\rho_{\mathrm{c}} \propto \beta^{1.5-2}$, a scaling well below analytic predictions as well as the higher-resolution one-dimensional simulations of BL08.

It is also possible that our approximations have underestimated the efficiency with which the bounce phase can redistribute the energy of vertical collapse to motions within the orbital plane, perhaps due to neglect of GR effects during phase III. Alternatively, the simulations of Laguna et al. (1993) may be altered by entropy production due to artificial viscosity, as suggested by the authors.

More recent hydrodynamical simulations of high- $\beta$ disruptions did not publish details on the spread of debris energy (Kobayashi et al. 2004; Guillochon et al. 2009), although we note that Kobayashi et al. (2004), using a one-dimensional mesh code, found results in qualitative agreement with BL08. On the other hand, Guillochon et al. (2009) 


\section{CHAPTER 4. STRONG COMPRESSION IN TDES}

found a vastly lower degree $\left(\rho_{\mathrm{c}} \approx 4 \rho_{*}\right)$ of central compression for a $\beta=7$ event than what is predicted analytically, by the affine model, or by one-dimensional hydrodynamic simulations, with the difference attributed to a combination of insufficient midplane resolution, the internal density gradient of the star, and the physical, three-dimensional effects discussed in $\S 5$. We have shown that some multidimensional effects are unlikely to be large due to the proximity of the sonic point to the region of maximum compression. Desynchronization due to density differences in the star, which will result in the core seeing a different "effective $\beta$ " than the rest of the star, may be an exception to this.

During the completion of this paper, an independent numerical study (Guillochon \& Ramirez-Ruiz 2013) was posted which found an approximate constancy in $\Delta \epsilon$ for $1 \lesssim \beta \lesssim 4$, in the course of a thorough investigation of low- $\beta$ TDEs. This result is broadly compatible with our paper, although further hydrodynamical simulations will be required to explore high $\beta$ values, and to test our prediction of desynchronized collapse for rapidly rotating, misaligned stars (which could restore a $\beta$ dependence to $\Delta \epsilon$, for deeply plunging disruptions). Like Guillochon \& Ramirez-Ruiz (2013), we argue that the assumption of frozen-in debris energy is made much more valid by assuming the energy freezes in when internal forces become negligible at $R=R_{\mathrm{t}}$, not at later times.

We note that the free solutions are of course an approximation to the physical reality of tidal disruption, and neglect a number of important physical effects. Some areas in particular need of further clarification by hydrodynamical simulation are the velocity and shape perturbations induced on the star by the nonlinear hydrodynamics of tidal disruption at $R \approx R_{\mathrm{t}}$. Although BL08 found these effects to generally be negligble for Phase II and III of a TDE, their simulations were one-dimensional and it is conceivable that in three dimensions the picture may change. The free solutions also 


\section{CHAPTER 4. STRONG COMPRESSION IN TDES}

fail to capture GR effects relevant for large $\beta$ values, although we have argued based on our own analysis and past literature that these effects do not qualitatively change our conclusions. Furthermore, we have not considered the effects of SMBH spin, which if misaligned with the orbital plane may be able to desynchronize stellar collapse.

Despite these limitations, the free solutions are a simple yet powerful method for considering the tidal free fall of disrupted bodies. Although we have focused on the disruption of main sequence stars by supermassive black holes, we stress that much of our analysis is general and applies equally well to other scenarios, such as tidal disruption of white dwarfs by intermediate mass black holes, or of planets or asteroids by more compact bodies. In all of these scenarios, the decoupling of vertical from in-plane motion will strongly compress the disrupted object, often by many orders of magnitude. In future work, we aim to apply the free solutions to these alternative physical regimes, as well as to internal motions such as pre-disruption rotation or post-disruption shock formation. 


\section{Chapter 5}

\section{Finite, Intense Accretion Bursts}

\section{from Tidal Disruption of Stars on}

\section{Bound Orbits}

K. Hayasaki, N. Stone, \& A. Loeb accepted in The Monthly Notices of the Royal Astronomical Society, 2013

\section{Abstract}

We study accretion processes for tidally disrupted stars approaching supermassive black holes on bound orbits, by performing three dimensional Smoothed Particle Hydrodynamics simulations with a pseudo-Newtonian potential. We find that there is a critical value of the orbital eccentricity below which all the stellar debris remains bound to the black hole. For high but sub-critical eccentricities, all the stellar mass is 


\section{CHAPTER 5. ECCENTRIC TIDAL DISRUPTIONS}

accreted onto the black hole in a finite time, causing a significant deviation from the canonical $t^{-5 / 3}$ mass fallback rate. When a star is on a moderately eccentric orbit and its pericenter distance is deeply inside the tidal disruption radius, there can be several orbit crossings of the debris streams due to relativistic precession. This dissipates orbital energy in shocks, allowing for rapid circularization of the debris streams and formation of an accretion disk. The resultant accretion rate greatly exceeds the Eddington rate and differs strongly from the canonical rate of $t^{-5 / 3}$. By contrast, there is little dissipation due to orbital crossings for the equivalent simulation with a purely Newtonian potential. This shows that general relativistic precession is crucial for accretion disk formation via circularization of stellar debris from stars on moderately eccentric orbits.

\subsection{Introduction}

Supermassive black holes (SMBHs) larger than $10^{5} M_{\odot}$ are now known to exist ubiquitously in galactic nuclei. SMBHs in nearby galaxies can be studied dynamically, whereas in more distant galaxies only the $\sim 1 \%$ of SMBHs undergoing major accretion episodes can be easily observed. The tidal disruption and subsequent accretion of a star by a SMBH, although intrinsically a rare event, is of observational interest as a way to probe dormant SMBHs in the local universe, because it can produce a powerful flare in excess of the Eddington luminosity (Carter \& Luminet 1983; Rees 1988; Evans \& Kochanek 1989; Strubbe \& Quataert 2009). These events are also of interest to low-frequency gravitational wave astronomy, as the stellar dynamical processes which funnel stars into the low angular momentum orbits necessary for tidal disruption events (TDEs) are similar to those which produce the extreme-mass ratio inspiral of a 


\section{CHAPTER 5. ECCENTRIC TIDAL DISRUPTIONS}

stellar-mass compact object onto a SMBH (Frank \& Rees 1976; Wang \& Merritt 2004; Madigan et al. 2011). TDEs have also been considered as part of the means by which a seed black hole grows into a SMBH (Zhao et al. 2002; Miralda-Escudé \& Kollmeier 2005; Bromley et al. 2012). All of these aspects of stellar tidal disruption have motivated past studies of TDEs.

The traditional picture of a TDE proceeds as follows: a star at large separation $(\sim 1 \mathrm{pc})$ approaches a SMBH on a nearly parabolic orbit. After the star is tidally disrupted by the black hole, half the stellar debris becomes gravitationally bound to the SMBH, because it loses orbital energy inside the tidal disruption radius. The bound debris falls back, and, after circularizing due to collisional shocks with other gas streams, accretes onto the black hole. Kepler's third law implies that the mass return rate decays with a $-5 / 3$ power of time (Rees 1988; Phinney 1989), asymptotically approaching zero. This simple analytic picture has been validated to the first order of approximation by hydrodynamical simulations (Evans \& Kochanek 1989), albeit with deviations from this law at early times (Lodato et al. 2009). Similar power-law behavior for the flare lightcurve is often assumed (i.e. $L \propto \dot{M}$ ), although here the theoretical evidence is less clear (Lodato \& Rossi 2011).

All-sky surveys in the X-ray and UV have so far observed 13 candidate tidal disruption flares (Komossa \& Bade 1999; Grupe et al. 1999; Greiner et al. 2000; Komossa et al. 2004; Halpern et al. 2004; Maksym et al. 2010; Bloom et al. 2011; Burrows et al. 2011). The observed light curves are in reasonable agreement with the theoretically predicted mass fallback rate of $t^{-5 / 3}$, although some show deviations (Burrows et al. 2011) and the number of samples is sufficiently small to make detailed testing of theoretical models difficult. A smaller number of strong TDE candidates have been 


\section{CHAPTER 5. ECCENTRIC TIDAL DISRUPTIONS}

found at optical wavelengths (van Velzen et al. 2011b; Gezari et al. 2012). Notably, two of the best-sampled TDEs differ strongly from the canonical theoretical picture: one possesses relativistic jets (Bloom et al. 2011; Zauderer et al. 2011), and the other lacks hydrogen lines in its spectra (Gezari et al. 2012).

It has been inferred from observations that the event rate of tidal disruption is $\sim 10^{-5} \mathrm{yr}^{-1}$ per galaxy (Donley et al. 2002). This observed rate is in rough agreement with uncertain theoretical rate estimates. In the standard scenario, stars are supplied to the SMBH by two body scattering, on a stellar relaxational timescale. Stars with angular momentum less than a critical value are in the phase space "loss cone" and are tidally destroyed on a dynamical time. Theoretical calculations indicate this rate to be $\sim 10^{-4}-10^{-6} \mathrm{yr}^{-1}$ for Milky Way-like galaxies, and that the peak flux into the loss cone comes from parsec scales. This motivates the assumption of nearly parabolic orbits (Magorrian \& Tremaine 1999; Wang \& Merritt 2004).

However, there are alternate sources of TDEs distinct from the standard two-body scattering model. Many of these feed stars to the SMBH at lower eccentricity. Our aim here is to quantify through hydrodynamical numerical simulations how the observable properties of tidal disruptions of stars on eccentric orbits deviate from the standard ones. In $\S 5.2$, we describe numerical procedures for simulating TDEs. We then analyze results of our simulations in $\S 5.3$. In $\S 5.4$ we consider nonstandard sources of TDEs and whether they can supply stars on low eccentricity orbits to a SMBH. Finally, $\S 5.5$ is devoted to summary and discussion of our scenario. 


\subsection{Numerical method}

In this section, we describe our procedures for numerically modeling the tidal disruption of stars on bound orbits. The simulations presented below were performed with a three-dimensional (3D) SPH code, which is a particle method that divides the fluid into a set of discrete "fluid elements" (i.e. particles), and is flexible in setting various initial configurations. The code is based on a version originally developed by Benz et al. (1990); Bate et al. (1995) and has been extensively used by many papers (e.g., Okazaki et al. (2002); Hayasaki et al. (2007)).

The SPH equations with the standard cubic-spline kernel are integrated using a second-order Runge-Kutta-Fehlberg integrator with individual time steps for each particle (Bate 1995), which results in enormous computational time savings when a large range of dynamical timescales are involved. We also use variable smoothing lengths scheme to calculate relevant spatial resolution in our code, but ignore the term proportional to the gradient of the smoothing length ${ }^{1}$ In the simulations shown in this paper, we adopt standard SPH artificial viscosity parameters $\alpha_{\mathrm{SPH}}=1$ or $\beta_{\mathrm{SPH}}=2$ and use no switch to limit the artificial viscosity.

We have performed two-stage simulations. We model a star as a polytropic gas sphere in hydrostatic equilibrium. The tidal disruption process is modeled by setting the

\footnotetext{
${ }^{1}$ term is introduced for calculating the gradient of fluid properties when the smoothing length is varied in space and time, and is important for ensuring energy conservation if the gradient of any physical quantities varies over a shorter length scale than the smoothing length (see Bate et al. 1995 for a review). In our simulations, the specific energy is conserved for all models except for Model 2a. This shows that the term plays no crucial role in our simulations.
} 
star in motion through the gravitational field of an SMBH.

\subsubsection{Formation of polytropes}

In our code, the polytrope is composed of an ensemble of gas particles, each of which has a mass chosen to be $10^{-5} M_{\odot}$ with a variable smoothing length. The particles are initially uniformly distributed in a spherical fashion, with an initial temperature $T_{\text {ini }}=1.2 \times 10^{6} \mathrm{~K}$. The initial spherical gas cloud is allowed to collapse under self-gravity using the polytropic equation of state:

$$
P=K \rho^{1+1 / n},
$$

where $n$ is the polytropic index and $K$ is assumed to be kept constant throughout the collapse. Note that $n=1.5$ corresponds to $\gamma=5 / 3$. The simulations continue over five dynamical times, where the dynamical time is defined by

$$
\Omega_{*}^{-1} \equiv \sqrt{\frac{r_{*}^{3}}{G m_{*}}} \simeq 5.1 \times 10^{-5}\left(\frac{r_{*}}{R_{\odot}}\right)^{3 / 2}\left(\frac{M_{\odot}}{m_{*}}\right)^{1 / 2} \mathrm{yr},
$$

where $G$ is Newton's constant and $r_{*}$ and $m_{*}$ are the stellar radius and mass, respectively.

Fig. 5.1 shows the radial density profile of the polytropic gas sphere at $4.4 \Omega^{-1}$, where the magnitude of ratio of thermal and gravitational energy becomes $\sim 0.5$. While circle marks show the radial density profile obtained from the SPH simulations, the solid line shows the profile obtained by numerically solving the Lane-Emden equation:

$$
\frac{1}{\xi^{2}} \frac{d}{d \xi}\left(\xi^{2} \frac{d \theta}{d \xi}\right)=-\theta^{n},
$$

where we define as dimensionless quantities, $\xi=r / r_{\mathrm{c}}$ with

$$
r_{\mathrm{c}}=\left(\frac{K(n+1)}{4 \pi G} \rho_{\mathrm{c}}^{1 / n-1}\right)^{1 / 2},
$$

and $\theta^{n}=\rho / \rho_{\mathrm{c}}$ with the central density $\rho_{\mathrm{c}}$. With boundary conditions that $\theta=1$ and 
$d \theta / d \xi=0$ at $\xi=0$ and $\theta=0$ and $\xi=R_{\odot} / r_{\mathrm{c}}$ at the surface of the star for 1 solar mass sun-type star, we obtained that $\rho_{c} \simeq 8.4 \mathrm{~g} \mathrm{~cm}^{-3}, r_{\mathrm{c}} \simeq 0.27 R_{\odot}$ and $K \simeq 2.5 \times 10^{15}$.

\subsubsection{Initial configuration}

A star is tidally disrupted when the tidal force of the black hole acting on the star is stronger than the self-gravitational force of the star. The radius where these two forces balance is defined as the tidal disruption radius by

$$
r_{\mathrm{t}}=\left(\frac{M_{\mathrm{BH}}}{m_{*}}\right)^{1 / 3} r_{*},
$$

where $M_{\mathrm{BH}}$ is the black hole mass.

Fig. 5.2 shows a density map of the polytropic gas sphere at $4.4 \Omega_{*}^{-1}$. The left panel shows the column density of polytropic sphere with $n=1.5$ over a range of five orders of magnitude. The right panel shows the star-black hole system on the $x-y$ plane, where both axes are normalized by $r_{\mathrm{t}}$ and the black hole is put on the origin of the system. The initial position of the star is given by $\boldsymbol{r}_{0}=\left(r_{0} \cos \phi_{0}, r_{0} \sin \phi_{0}, 0\right)$, where $\left|\boldsymbol{r}_{0}\right|=r_{0}$ is the radial distance from the black hole and $\phi_{0}$ shows the angle between $x$-axis and $\boldsymbol{r}_{0}$. The star within a small square is zoomed out to the whole left panel. Note that the stellar size on the plot has been scaled up by a factor of a few for visual clarity.

The motion of a test particle in the central SMBH potential $U(r)$ admits two conserved quantities. The radial velocity and angular velocity are given by energy conservation and angular momentum conservation as

$$
\begin{aligned}
\dot{r} & =\sqrt{2(\epsilon-U(r))-\frac{l^{2}}{r^{2}}}, \\
\dot{\phi} & =\frac{l}{r^{2}},
\end{aligned}
$$




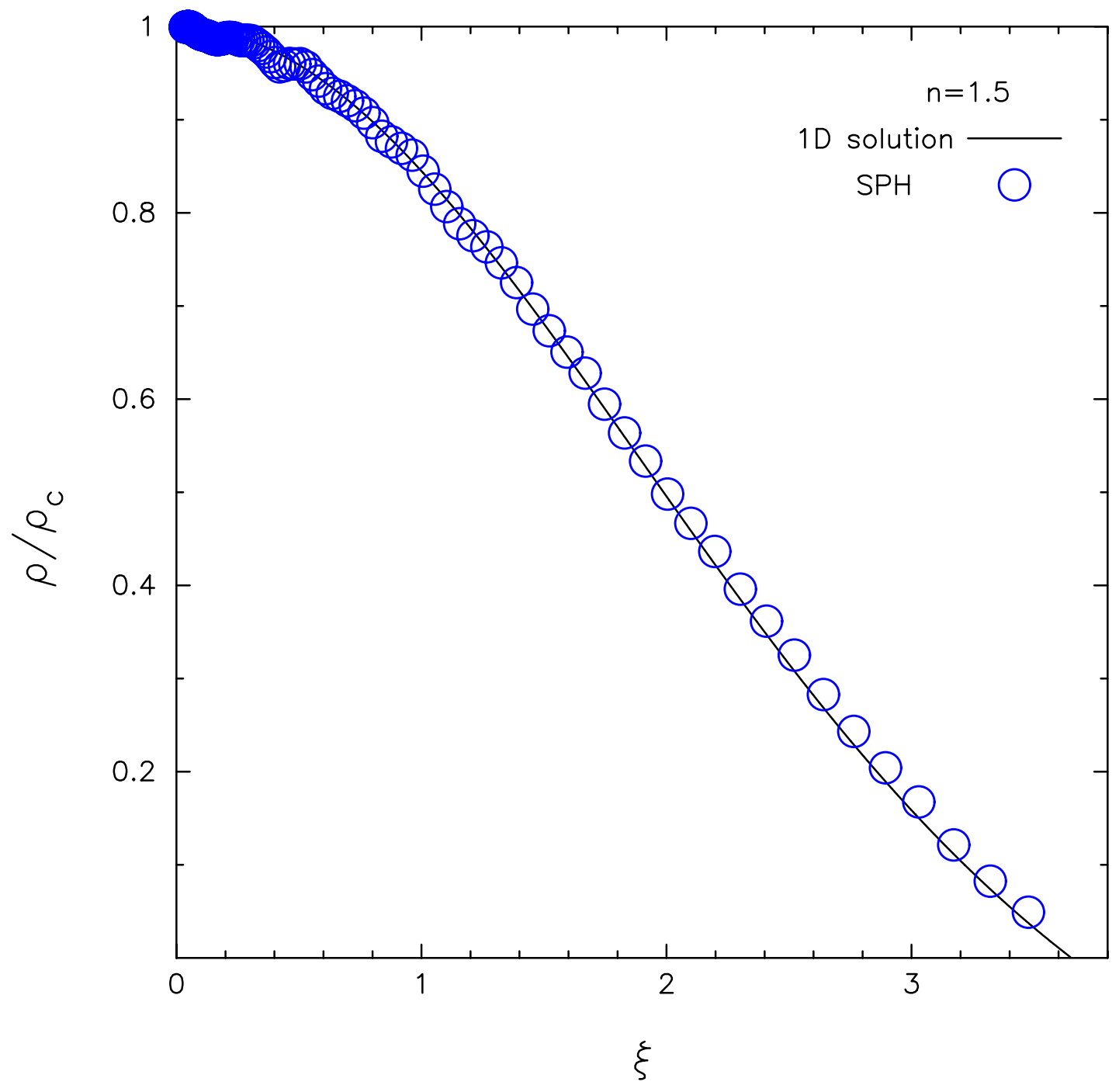

Figure 5.1.- : The radial density distribution of the polytropic gas sphere with $n=1.5$. The solid line and circle marks show the solution for the Lane-Emden equation with $n=1.5$ and the solution derived from the SPH simulation, respectively. 
where $\epsilon$ and $l$ are the specific energy and the specific angular momentum, respectively.

For bound orbits,

$$
\begin{aligned}
\epsilon & =\frac{\left(r_{\mathrm{p}} / r_{\mathrm{a}}\right)^{2} U\left(r_{\mathrm{p}}\right)-U\left(r_{\mathrm{a}}\right)}{\left(r_{\mathrm{p}} / r_{\mathrm{a}}\right)^{2}-1}, \\
l & =\sqrt{2 r_{\mathrm{p}}^{2}\left(\epsilon-U\left(r_{\mathrm{p}}\right)\right)}=\sqrt{2 r_{\mathrm{a}}^{2}\left(\epsilon-U\left(r_{\mathrm{a}}\right)\right)},
\end{aligned}
$$

where $r_{\mathrm{p}}$ and $r_{\mathrm{a}}$ are the pericenter distance and the apocenter distance, respectively. Therefore, the initial velocity vector is given by $\boldsymbol{v}_{0}=\left(\dot{r}\left(r_{0}\right) \cos \phi_{0}-\right.$ $\left.r_{0} \dot{\phi}\left(\phi_{0}\right) \sin \phi_{0}, \dot{r}\left(r_{0}\right) \sin \phi_{0}+r_{0} \dot{\phi}\left(\phi_{0}\right) \cos \phi_{0}, 0\right)$.

In our simulations, the black hole is represented by a sink particle with the appropriate gravitational mass $M_{\mathrm{BH}}$. All gas particles that fall within a specified accretion radius are accreted by the sink particle. We set the accretion radius of the black hole as equal to the Schwarzshild radius $r_{\mathrm{S}}=2 G M_{\mathrm{BH}} / c^{2}$, with $c$ being the speed of light.

\subsubsection{Treatment of relativistic precession}

In order to treat approximately the relativistic precession of a test particle in the Schwarzschild metric, we incorporate into our SPH code the following pseudo-Newtonian potential (Wegg 2012):

$$
U(r)=-\frac{G M_{\mathrm{BH}}}{r}\left[c_{1}+\frac{1-c_{1}}{1-c_{2}\left(r_{\mathrm{S}} / 2 r\right)}+c_{3} \frac{r_{\mathrm{S}}}{2 r}\right],
$$

where we adopt that $c_{1}=(-4 / 3)(2+\sqrt{6}), c_{2}=(4 \sqrt{6}-9)$, and $c_{3}=(-4 / 3)(2 \sqrt{6}-3)$. Equation (5.10) reduces to the Newtonian potential when $c_{1}=1$ and $c_{2}=c_{3}=0$, while it reduces to the well-known Paczynski \& Wiita pseudo-Newtonian potential (Paczyńsky $\&$ Wiita 1980) when $c_{1}=c_{3}=0$. Note that equation (5.10) includes no higher-order 


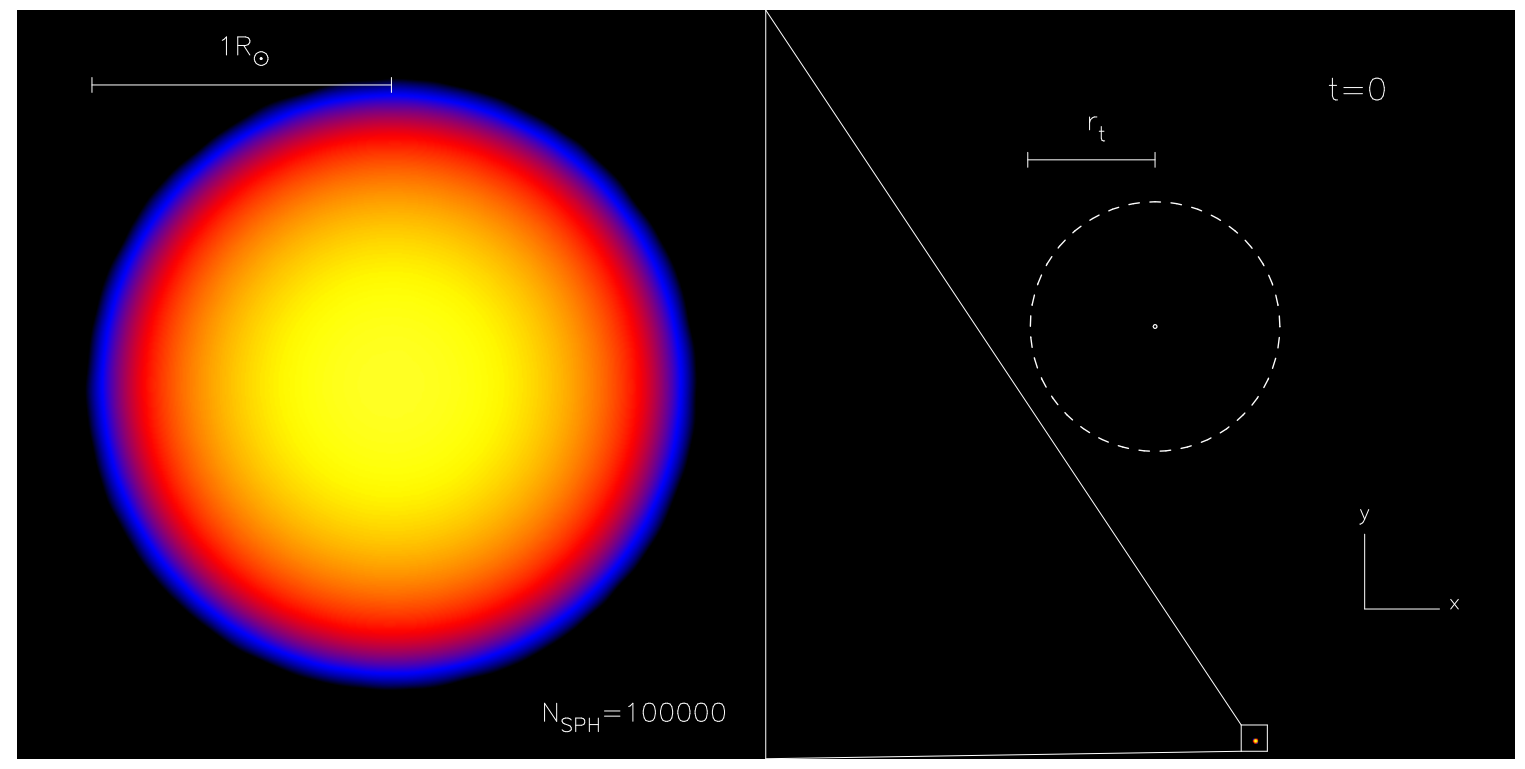

Figure 5.2.- Initial configuration of our simulations. The left panel shows a density map of the polytropic gas sphere with $n=1.5$. Its radius is $1 R_{\odot}$ and the number of SPH particles $N_{\mathrm{SPH}}$ are annotated in the right-bottom corner of the panel. The panel on the right-hand-side shows the initial position of the star on the $x-y$ plane. The small square corresponds to the entire left panel, the dashed circle shows the tidal disruption radius $r_{\mathrm{t}}$ and the black hole is positioned at the center. The simulation run time $t$ in the right-top corner is normalized by $\Omega_{*}^{-1}$. In the right panel, the star is initially located at $\left(r_{0} \cos \phi_{0}, r_{0} \sin \phi_{0}\right)$, where $r_{0}=3 r_{\mathrm{t}}$ and $\phi_{0}=-0.4 \pi$. 


\section{CHAPTER 5. ECCENTRIC TIDAL DISRUPTIONS}

relativistic effects such as the black hole spin or gravitational wave emission.

Fig. 5.3 tests how accurately the SPH particles composing the star follow test particle motion in the Schwarzschild metric. Specifically, the figure shows the motion of three particles orbiting the black hole with $(e, \beta)=(0.8,5)$, where $\beta=r_{\mathrm{t}} / r_{\mathrm{p}}$ is the penetration factor which determines how deeply the star plunges into the black hole potential inside the tidal disruption radius. The central point and dashed circle show the black hole and the tidal disruption radius, respectively. The small crosses, dotted line, and solid line represent the geodesics of the Schwarzschild metric, orbits of the test particle under the pseudo-Newtonian potential, and those of a SPH particle in the simulation of Model 2a, respectively.

During the first one and a half orbits, the test particle is in good agreement with the corresponding geodesic. The subsequent deviation from the geodesic is due to the relatively low eccentricity and high $\beta$, since equation (5.10) is tailored to parabolic orbits with lower $\beta$ (Wegg 2012, see also Fig. 5.4). The first three and half orbits of the test particle are in rough agreement with corresponding orbits of the SPH particle. Subsequent large deviations originate from orbital circularization via shock-induced energy dissipation (see Section 5.3.2 in detail), and although our model becomes unreliable at this point, it is clear that stream crossing is leading to rapid energy dissipation.

Fig. 5.4 shows the dependence of the error rate on the eccentricity of a test particle moving under the pseudo-Newtonian potential given by equation (5.10). The error rate is defined by $\left(\Delta \phi_{\mathrm{GR}}-\Delta \phi_{\mathrm{PN}}\right) / \Delta \phi_{\mathrm{GR}} \times 100$, where $\Delta \phi_{\mathrm{GR}}$ and $\Delta \phi_{\mathrm{PN}}$ are the precession angles corresponding to equation (2) and (4) of Wegg (2012), respectively, with a given 


\section{CHAPTER 5. ECCENTRIC TIDAL DISRUPTIONS}

specific angular momentum, specific orbital energy, and equation (5.10). The solid line, dashed line, and dotted line show error rates of an orbit with $r_{\mathrm{p}}=3 r_{\mathrm{S}}(\beta \approx 7.9)$, $r_{\mathrm{p}}=5 r_{\mathrm{S}}(\beta \approx 4.7)$, and $r_{\mathrm{p}}=10 r_{\mathrm{S}}(\beta \approx 2.4)$, respectively, where $r_{\mathrm{t}} \approx 23.6 r_{\mathrm{S}}$ is adopted. The error rate increases as the eccentricity is lower and the penetration factor is higher. From the figure, the error rate is estimated to be roughly $15 \%$ for $e=0.8$ with $\beta=5$, while it is less than $1 \%$ for $e \gtrsim 0.95$ with arbitrary $\beta$.

\subsubsection{Numerical models}

We have performed eight simulations of tidal disruption events with different parameters. The common parameters through all of simulations are following: $m_{*}=1 M_{\odot}, r_{*}=1 R_{\odot}$, $M_{\mathrm{BH}}=10^{6} M_{\odot}, \phi_{0}=-0.4 \pi$, and $\gamma=5 / 3$. The total number of SPH particles used in each simulation are $10^{5}$, and the termination time of each simulation is $4 \Omega_{*}^{-1}$. Table 1 summarizes each model. Model 1a shows the standard TDE under the Newtonian potential, while Model 1b has the same simulation parameters as Model 1a, except that the star moves under the pseudo-Newtonian potential given by equation (5.10). Model 1c and 1d have the same parameters as Model 1a and Model1b, respectively, but for $\beta=5$. Model 2a has the same parameters as Model 1d except that the star is on an eccentric orbit, with $e=0.8$. Model $2 \mathrm{~b}$ is the same parameters as Model 2a except that the star moves under the Newtonian potential. Model 3a has the same parameters as Model 2a but for $e=0.98$ and $\beta=1$, and Model 3b has the same parameters as Model 3a but for $\beta=5$. 


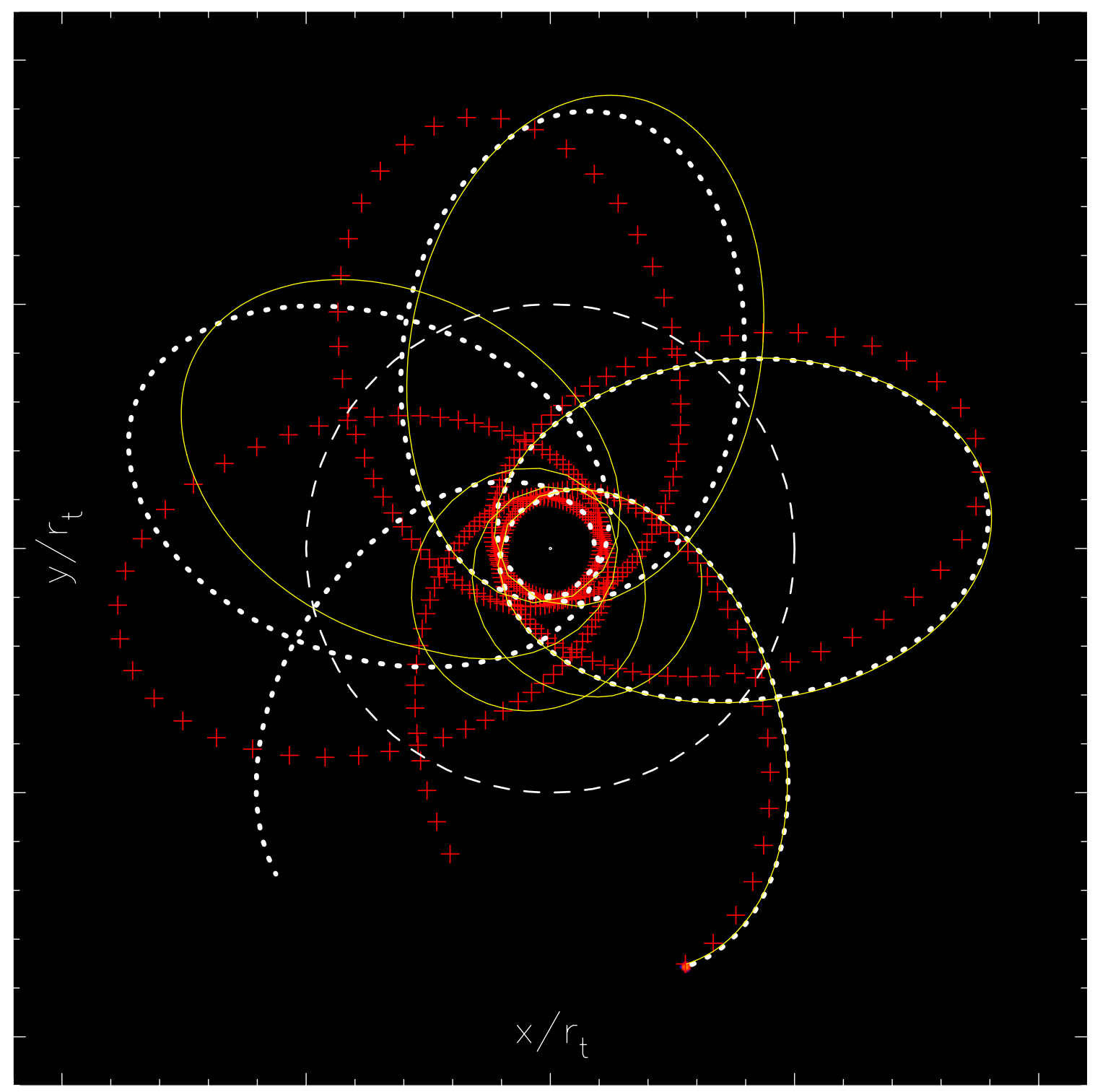

Figure 5.3.- Motion of three particles with orbital parameters $(e, \beta)=(0.8,5)$ in the $x-y$ plane. Each axis is normalized by $r_{\mathrm{t}}$ given by equation (5.5). The central point and dashed circle represent the black hole and tidal disruption radius, respectively. The small crosses, dotted line, and solid line show the geodesics in the Schwarzshild metric, orbits of a test particle under the pseudo-Newtonian potential given by equation (5.10), and orbits of a SPH particle in the simulation of Model 2a (see text of $\S 5.3 .2$ ), respectively. 


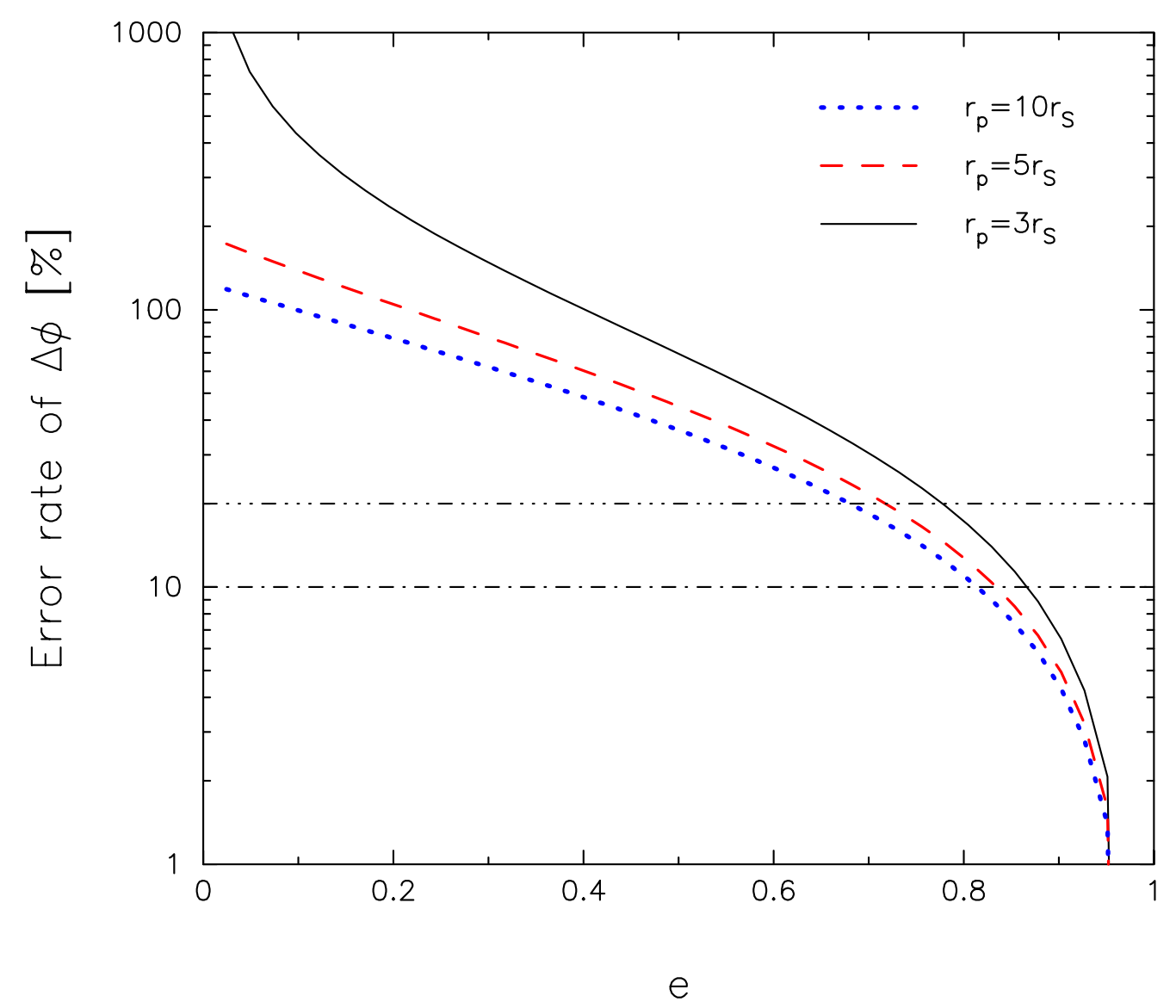

Figure 5.4.-: Dependence of error rate of precession angle $\Delta \phi$ on the eccentricity of a test particle moving under the pseudo-Newtonian potential given by equation (5.10). The solid line, dashed line, and dotted line show error rates of an orbit with $r_{\mathrm{p}}=3 r_{\mathrm{S}}, r_{\mathrm{p}}=5 r_{\mathrm{S}}$, and $r_{\mathrm{p}}=10 r_{\mathrm{S}}$, respectively. The horizontal dash-dotted line and dash-three-dotted line show the error cutoffs of $10 \%$ and $20 \%$, respectively. 
Table 5.1:: The first column shows each simulated scenario. The second, third, fourth, and fifth columns are the penetration factor $\beta=r_{\mathrm{p}} / r_{\mathrm{T}}$, the initial orbital eccentricity $e_{*}$, the initial semi-major axis $a_{*}$, and the radial distance between the black hole and the initial position of the star, respectively. The last column describes the remark for each model.

\begin{tabular}{cccccc}
\hline Model & $\beta=r_{\mathrm{t}} / r_{\mathrm{p}}$ & $e_{*}$ & $a_{*}\left[r_{\mathrm{t}}\right]$ & $r_{0}\left[r_{\mathrm{t}}\right]$ & Remarks \\
\hline $1 \mathrm{a}$ & 1 & 1.0 & - & 3 & Newtonian \\
$1 \mathrm{~b}$ & 1 & 1.0 & - & 3 & Pseudo-Newtonian \\
$1 \mathrm{c}$ & 5 & 1.0 & - & 3 & Newtonian \\
$1 \mathrm{~d}$ & 5 & 1.0 & - & 3 & Pseudo-Newtonian \\
2a & 5 & 0.8 & 1.0 & 1.8 & Pseudo-Newtonian \\
$2 \mathrm{~b}$ & 5 & 0.8 & 1.0 & 1.8 & Newtonian \\
3a & 1 & 0.98 & 50.0 & 3 & Pseudo-Newtonian \\
3b & 5 & 0.98 & 10.0 & 3 & Pseudo-Newtonian \\
\hline
\end{tabular}




\subsection{Tidal disruption of stars on bound orbits}

We first describe the evolution of a tidally disrupted star for standard TDEs $(e=1)$. Next, the process of tidal disruption for a star on a fairly eccentric orbit $(e=0.98)$ is presented. Finally, accretion disk formation in relatively low eccentricity TDEs $(e=0.8)$ is presented.

As an approaching star enters into the tidal disruption radius, its fluid elements become dominated by the tidal force of the black hole, while their own self-gravity and pressure forces become relatively negligible. The tidal force then produces a spread in specific energy of the stellar debris

$$
\Delta \epsilon \approx \frac{G M_{\mathrm{BH}} r_{*}}{r_{\mathrm{t}}^{2}}
$$

The total mass of the stellar debris is defined with the differential mass distribution $m(\epsilon) \equiv d M(\epsilon) / d \epsilon$ by

$$
M(\epsilon) \equiv \int_{-\infty}^{\infty} m\left(\epsilon^{\prime}\right) d \epsilon^{\prime}
$$

When a star is disrupted from a parabolic orbit, $m(\epsilon)$ will be centered on zero and distributed over $-\Delta \epsilon \leq \epsilon \leq \Delta \epsilon$.

Since the stellar debris with negative specific energy is bounded to the black hole, it returns to pericenter and will eventually accrete onto the black hole. If its specific energy is approximately equal to the specific binding energy, $\epsilon \approx-G M_{\mathrm{BH}} / 2 a$ with the semi-major axis of the stellar debris $a$, the mass fallback rate is then defined by, e.g., Evans \& Kochanek (1989)

$$
\frac{d M}{d t}=\frac{d M(\epsilon)}{d \epsilon}\left|\frac{d \epsilon}{d t}\right| \quad(\epsilon<0),
$$


where

$$
\frac{d \epsilon}{d t}=-\frac{1}{3}\left(2 \pi G M_{\mathrm{BH}}\right)^{2 / 3} t^{-5 / 3}
$$

This is derived from the relationship between the orbital period and the specific energy:

$$
t=\frac{\pi}{\sqrt{2}} \frac{G M_{\mathrm{BH}}}{\epsilon^{3 / 2}}
$$

from Kepler's third law. The standard $d M / d t \propto t^{-5 / 3}$ scaling then arises with the assumption that $d M / d \epsilon$ is constant, or at least asymptotes to a constant value at late times. From equation (5.15), the orbital period of the most tightly bound orbit $\left(t_{\min }\right)$ and that of the most loosely bound orbit $\left(t_{\max }\right)$ are given by

$$
t \rightarrow\left\{\begin{array}{ll}
t_{\min }=(\pi / \sqrt{2})\left(M_{\mathrm{BH}} / m_{*}\right)^{1 / 2} \Omega_{*}^{-1} & (\epsilon=\Delta \epsilon) \\
t_{\max }=\infty & (\epsilon=0)
\end{array} .\right.
$$

Figs. 5.5-5.8 show the evolution of differential mass distributions and their corresponding mass fallback rates in Models 1a-1d. While the differential mass distribution is shown in panel (a), the mass fallback rate is shown in panel (b). In both panels, the solid line, dashed line, dot-dashed line, and dotted line represent the mass distributions and corresponding mass fallback rates at $t=0.0, t=0.37, t=0.79$, and $t=4.0$, respectively. Note that $t=0.5$ corresponds to the time when the star reaches pericenter. In panel (b), the horizontal solid line denotes the Eddington rate:

$$
\begin{aligned}
\dot{M}_{\mathrm{Edd}} & =\frac{1}{\eta} \frac{L_{\mathrm{Edd}}}{c^{2}} \simeq 2.2 \times 10^{-2} \\
& \times\left(\frac{\eta}{0.1}\right)^{-1}\left(\frac{M_{\mathrm{BH}}}{10^{6} M_{\odot}}\right) M_{\odot} \mathrm{yr}^{-1}
\end{aligned}
$$

where $L_{\mathrm{Edd}}=4 \pi G M_{\mathrm{BH}} m_{\mathrm{p}} c / \sigma_{\mathrm{T}}$ is the Eddington luminosity with $m_{\mathrm{p}}$ and $\sigma_{\mathrm{T}}$ denoting the proton mass and Thomson scattering cross section, respectively, and $\eta$ is the mass-to-energy conversion efficiency, which is set to 0.1 in the following discussion. 


\section{CHAPTER 5. ECCENTRIC TIDAL DISRUPTIONS}

In Model 1a, the mass distribution broadens with time as the stellar debris is put on near-ballistic orbits by tidal interaction with the black hole. In panel (a), the central peak formed after $t \gtrsim 1.0$ is due to mass congregation, from the self-gravity of the stellar debris (in this scenario, the star is barely disrupted). The energy spread corresponds to $\Delta \epsilon$ before and after the tidal disruption. The corresponding mass fallback rates are proportional to $t^{-5 / 3}$ except for the solid line in panel (b). This is in good agreement with the literatures (Rees 1988; Evans \& Kochanek 1989). The very slight deviation from $t^{-5 / 3}$ originates from the convexity around $\Delta \epsilon$ due to re-congregation of stellar mass under self-gravity - see also Lodato et al. (2009).

Model 1b has the same simulation parameters as Model 1a except that it adopts the pseudo-Newtonian potential. In panel (a) of Fig. 5.6, the final energy spread and the central peak are slightly wider and milder than those of Model 1a. This is because the energy imparted by the tidal force under the pseudo-Newtonian potential is slightly larger than that of the Newtonian potential. From equation (5.10), the energy spread under the pseudo-Newtonian potential is evaluated to be

$$
\left.\Delta \epsilon_{\mathrm{PN}} \approx \frac{d U(r)}{d r}\right|_{r=r_{\mathrm{t}}} r_{*}=\Delta \epsilon\left[c_{1}+\frac{1-c_{1}}{\left[1-c_{2}\left(r_{\mathrm{S}} / 2 r_{\mathrm{t}}\right)\right]^{2}}+2 c_{3} \frac{r_{\mathrm{S}}}{2 r_{\mathrm{t}}}\right] .
$$

The expected energy spread $\Delta \epsilon_{\mathrm{PN}} \sim 1.1 \Delta \epsilon$ for $10^{6} M_{\odot}$ is in good agreement with the simulated energy spread.

Model 1c has the same simulation parameters as Model 1a except for $\beta=5$, while Model 1d has the same simulation parameters as Model 1c except for adopting the pseudo-Newtonian potential. For higher value of $\beta$ than unity, the tidal forces acting on the star become stronger because the pericenter distance is smaller than the tidal disruption radius. 
CHAPTER 5. ECCENTRIC TIDAL DISRUPTIONS

From equation (5.11), the energy spread of the stellar debris is formally proportional to the square of $\beta$, and should become 25 times larger for Model 1c and Model 1d than that of $\beta=1$. In these two models, however, the energy spread is only about 1.4 times wider than that of standard model, showing that the energy spread weakly depends on $\beta$. Such a weak dependence of the energy spread on $\beta$ was found in the context of the binary disruption by Sari et al. (2010). This is also in agreement with recent analytic (Stone et al. 2012b) and numerical work (Guillochon \& Ramirez-Ruiz 2013).

Since the tidal disruption is less marginal as $\beta$ is higher, the re-congregation of the mass due to self-gravity of the stellar debris is prevented. This leads to the mildly-sloped mass distribution, and therefore the peak of the mass fallback rate also smooths. There is no remarkable difference between Model 1c and Model 1d except that the energy spread of Model 1d is slightly wider than that of Model 1c following equation (5.18). 


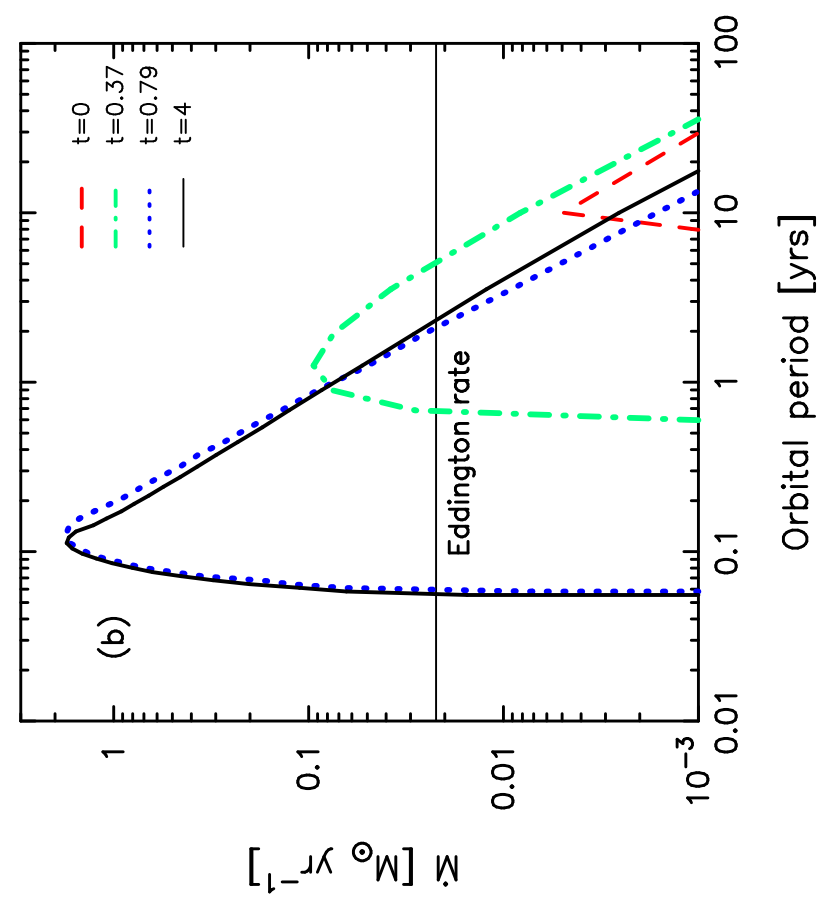

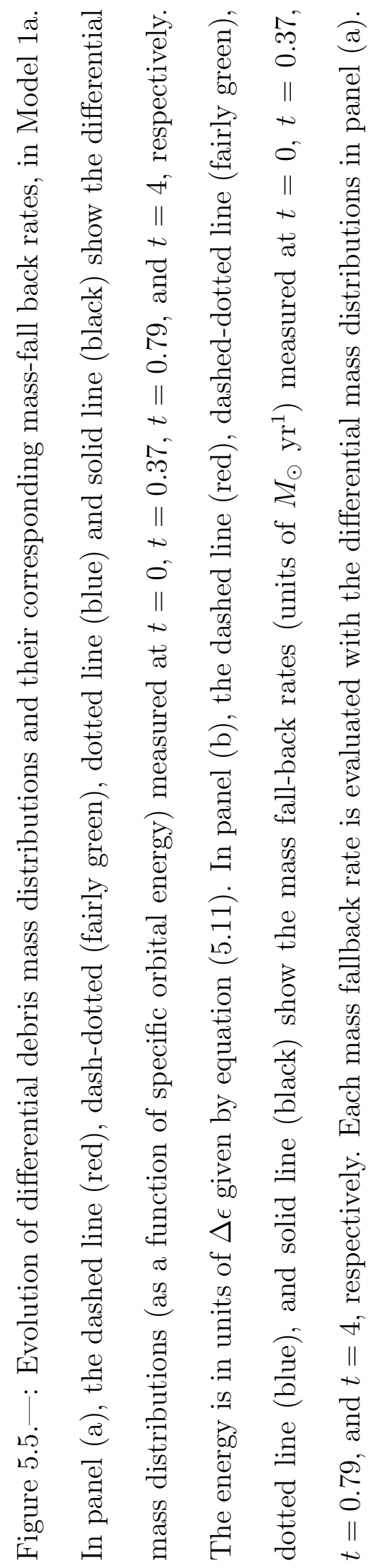

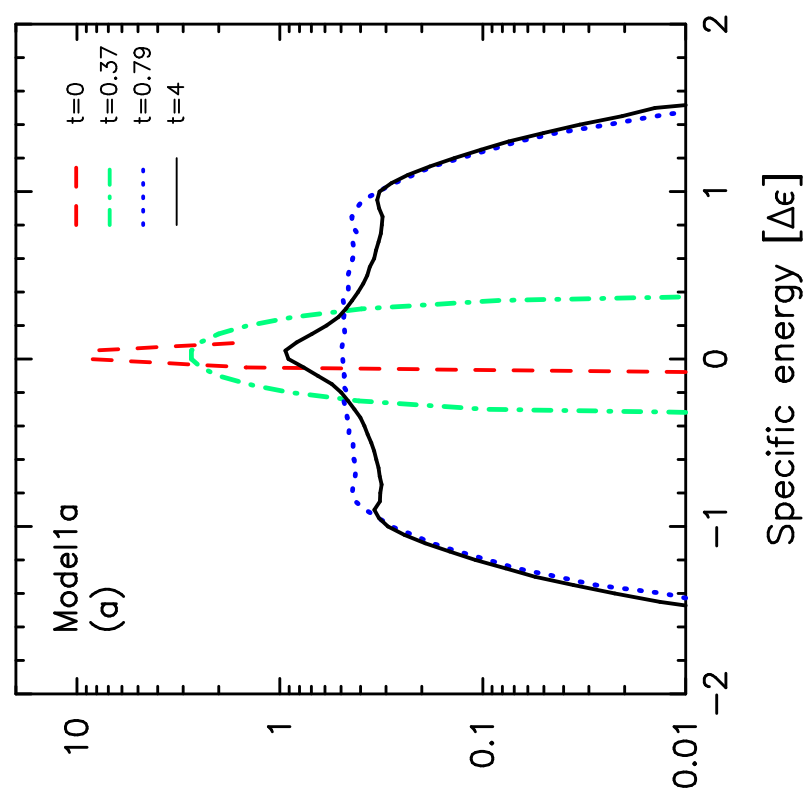

$\left[\ni \nabla /{ }^{*} \mathrm{U}\right] \quad \ni \mathrm{эP} /(\ni) \mathrm{WP}$ 


\subsubsection{Eccentric tidal disruption}

The specific orbital energy of a star on an eccentric orbit is given by

$$
\epsilon_{\text {orb }} \approx-\frac{G M_{\mathrm{BH}}}{2 a_{*}}=-\frac{G M_{\mathrm{BH}}}{2 r_{\mathrm{t}}} \beta\left(1-e_{*}\right) .
$$

This quantity is less than zero because of the finite value of $a_{*}$, in contrast to the standard, parabolic orbit of a star. If $\epsilon_{\text {orb }}$ is less than $\Delta \epsilon$, all the stellar debris should be bounded to the black hole, even after the tidal disruption. The condition $\epsilon_{\text {orb }}=\Delta \epsilon$ therefore gives a critical value of orbital eccentricity of the star

$$
e_{\mathrm{crit}} \approx 1-\frac{2}{\beta}\left(\frac{m_{*}}{M_{\mathrm{BH}}}\right)^{1 / 3},
$$

below which all the stellar debris should remain gravitationally bound to the black hole (Amaro-Seoane et al. 2012a). The critical eccentricity is evaluated to be $e_{\text {crit }}=0.996$ for Model 2a and Model 3b, whereas $e_{\text {crit }}=0.98$ for Model 3a.

For the eccentric TDEs, $t_{\min }$ and $t_{\max }$ are obtained by substituting $\epsilon=\Delta \epsilon \pm \epsilon_{\text {orb }}$ into equation (5.15). The orbital period of the most tightly bound orbit is given by

$$
t_{\min }=\frac{\pi}{\sqrt{2}} \frac{\Omega_{*}^{-1}}{\beta^{3 / 2}\left(1-e_{*}\right)^{3 / 2}} .
$$

The orbital period of the most loosely bound orbit $t_{\max }$ is estimated to be $\infty$ for $e_{*} \geq e_{\text {crit }}$, while $t_{\max }$ converges with

$$
t_{\max }=\frac{\pi}{\sqrt{2}} \Omega_{*}^{-1}\left[\frac{\beta\left(1-e_{*}\right)}{2}-\left(\frac{m_{*}}{M_{\mathrm{BH}}}\right)^{1 / 3}\right]^{-3 / 2}
$$

for $e_{*}<e_{\text {crit }}$. The duration time of mass fallback for eccentric TDEs with $e_{*}<e_{\text {crit }}$ is 

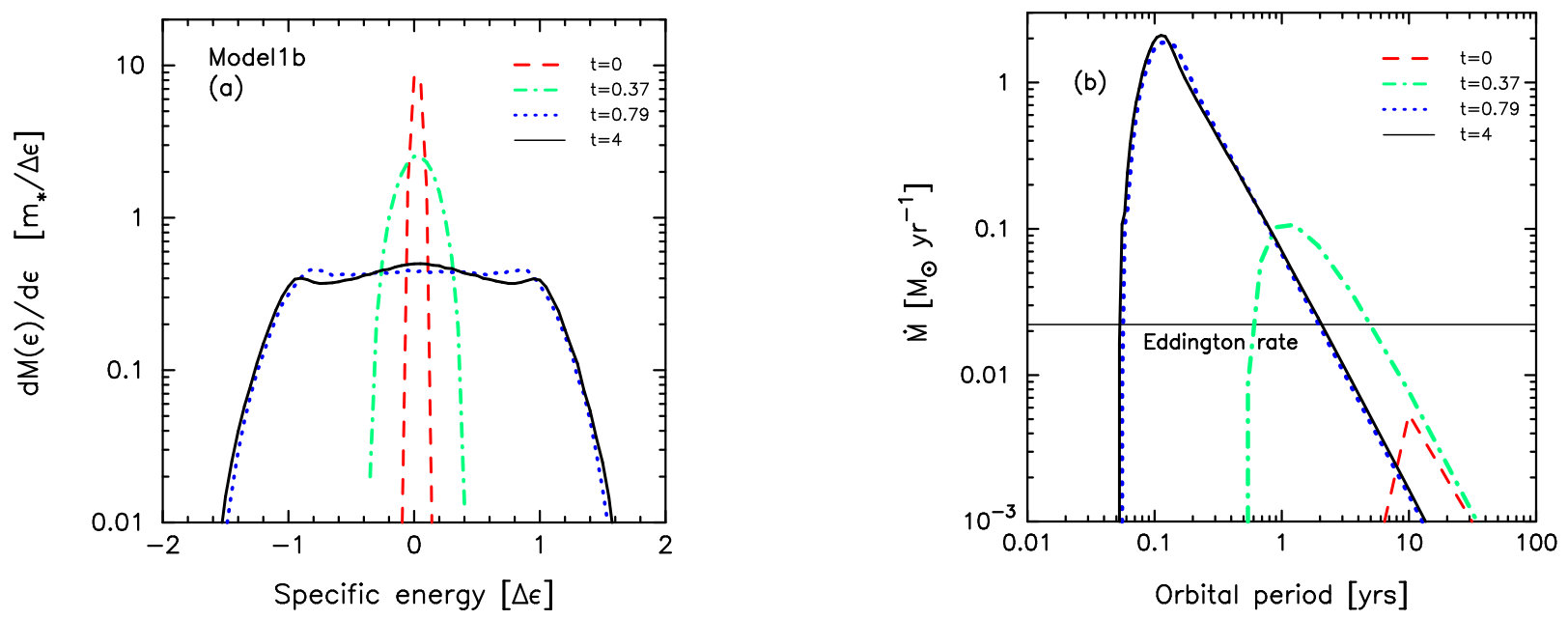

Figure 5.6.-: Same as Fig. 5.5, but for Model 1b.
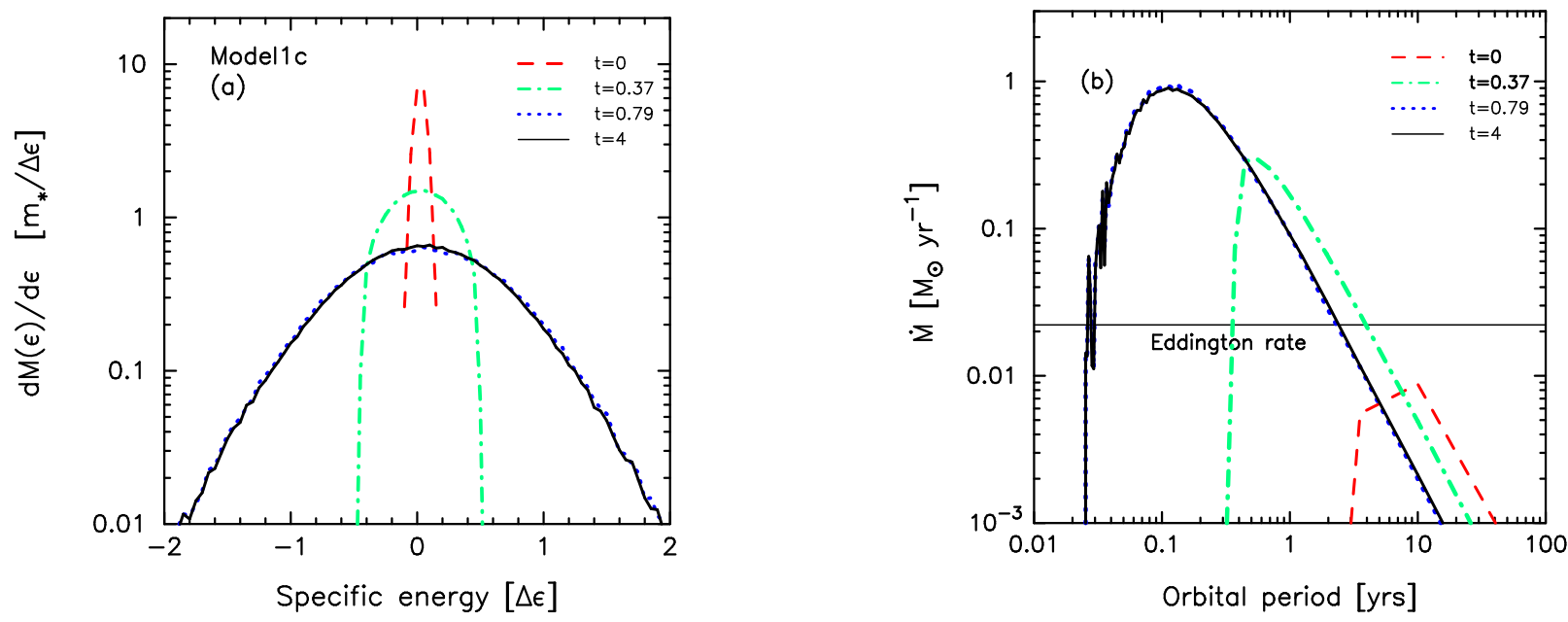

Figure 5.7.- : Same as Fig. 5.5, but for Model 1c. 
thus predicted to be finite and can be written by

$$
\begin{aligned}
\Delta t & =t_{\max }-t_{\min }=\frac{\pi}{\sqrt{2}} \frac{\Omega_{*}^{-1}}{\left[\beta\left(1-e_{*}\right)\right]^{3 / 2}} \\
& \times\left(\left[\frac{1}{2}-\frac{1}{\beta\left(1-e_{*}\right)}\left(\frac{m_{*}}{M_{\mathrm{BH}}}\right)^{1 / 3}\right]^{-3 / 2}-1\right) .
\end{aligned}
$$

Evaluating this gives $\Delta t \approx 4.3 \Omega_{*}^{-1}$ for Model $2 \mathrm{a}$ and $\Delta t \approx 207 \Omega_{*}^{-1}$ for Model $3 \mathrm{~b}$, whereas $\Delta t \rightarrow \infty$ for Model 3a.

The characteristic mass fallback rate for eccentric TDEs is given by

$$
\begin{aligned}
\dot{M}_{\text {ecc,fallback }} & =\frac{m_{*}}{\Delta t}=\frac{\sqrt{2}}{\pi} \frac{m_{*}}{\Omega_{*}^{-1}} \frac{1}{\left[\beta\left(1-e_{*}\right)\right]^{-3 / 2}} \\
& \times\left(\left[\frac{1}{2}-\frac{1}{\beta\left(1-e_{*}\right)}\left(\frac{m_{*}}{M_{\mathrm{BH}}}\right)^{1 / 3}\right]^{-3 / 2}-1\right)^{-1} .
\end{aligned}
$$

Figs. 5.9 and 5.10 show the evolution of differential mass distributions and corresponding mass fallback rates in Model 3a and Model 3b, respectively. The figure formats are the same as Figs. 5.5-5.8. The mass is not distributed around zero but around $-\Delta \epsilon$ in Model $3 \mathrm{a}$, and around $-5 \Delta \epsilon$ in Model 3b. This is because the specific energy of the initial stellar orbit is originally negative (see equation 5.19). It is clear from the negative shift of the mass distribution's center that most of the mass in Model 3a and all of the mass in Model 3b are bounded. As shown in panel (a) of Fig. 5.9, the resultant energy spread is slightly larger than we analytically expected. This suggests that the critical eccentricity is slightly smaller than the value in equation (5.20).

From equations (5.21) and (5.22), $t_{\min }=0.04 \mathrm{yr}$ and $t_{\max } \rightarrow \infty$ for Model 3a, and $t_{\min }=0.0035 \mathrm{yr}$ and $t_{\max }=0.014 \mathrm{yr}$ for Model $3 \mathrm{~b}$. These values deviate from simulation results, because they were derived assuming a smaller spread in energy than in our simulations. Furthermore, $t_{\min }$ is shorter than that of the parabolic case. This makes the 


\section{CHAPTER 5. ECCENTRIC TIDAL DISRUPTIONS}

mass fallback rate about one order of magnitude higher. Notably, the mass accretion is completely finite in Model 3b and its rate is enhanced by about two orders of magnitude, because its timescale is much shorter than that of the standard model. 

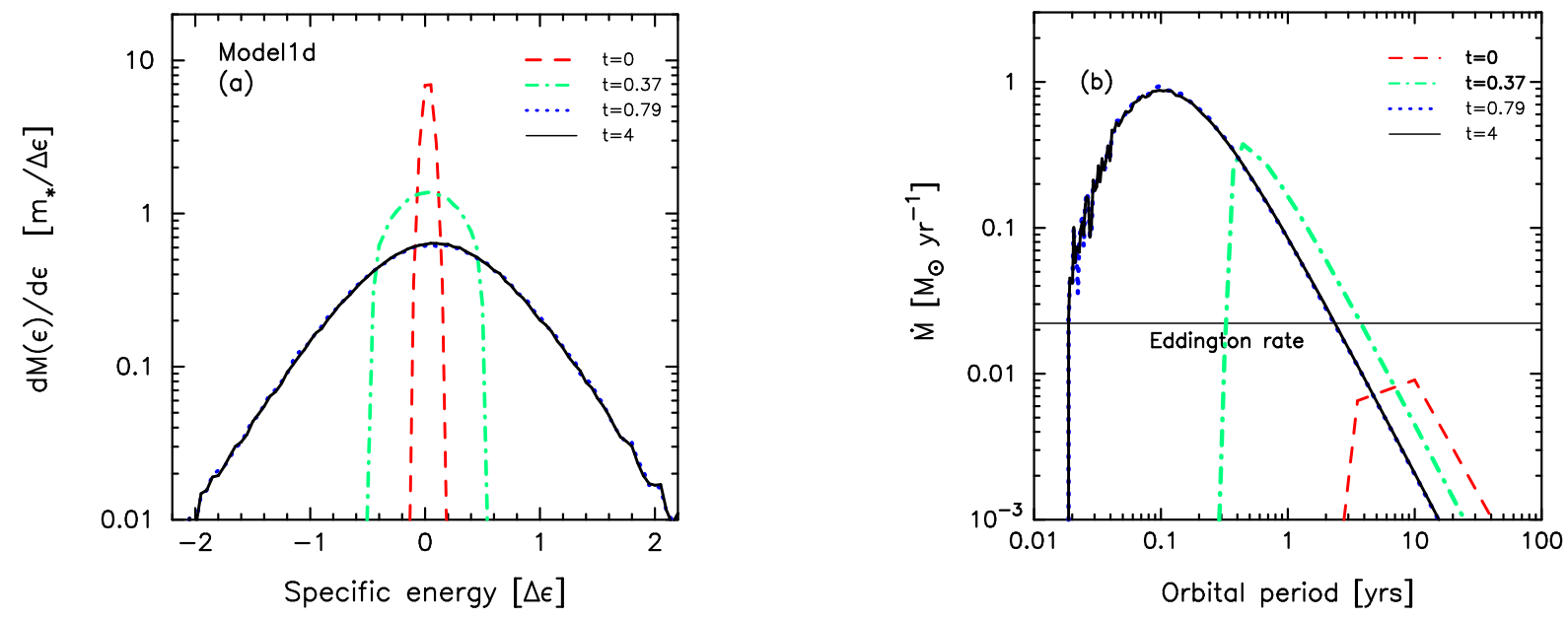

Figure 5.8.- : Same as Fig. 5.5, but for Model 1d.
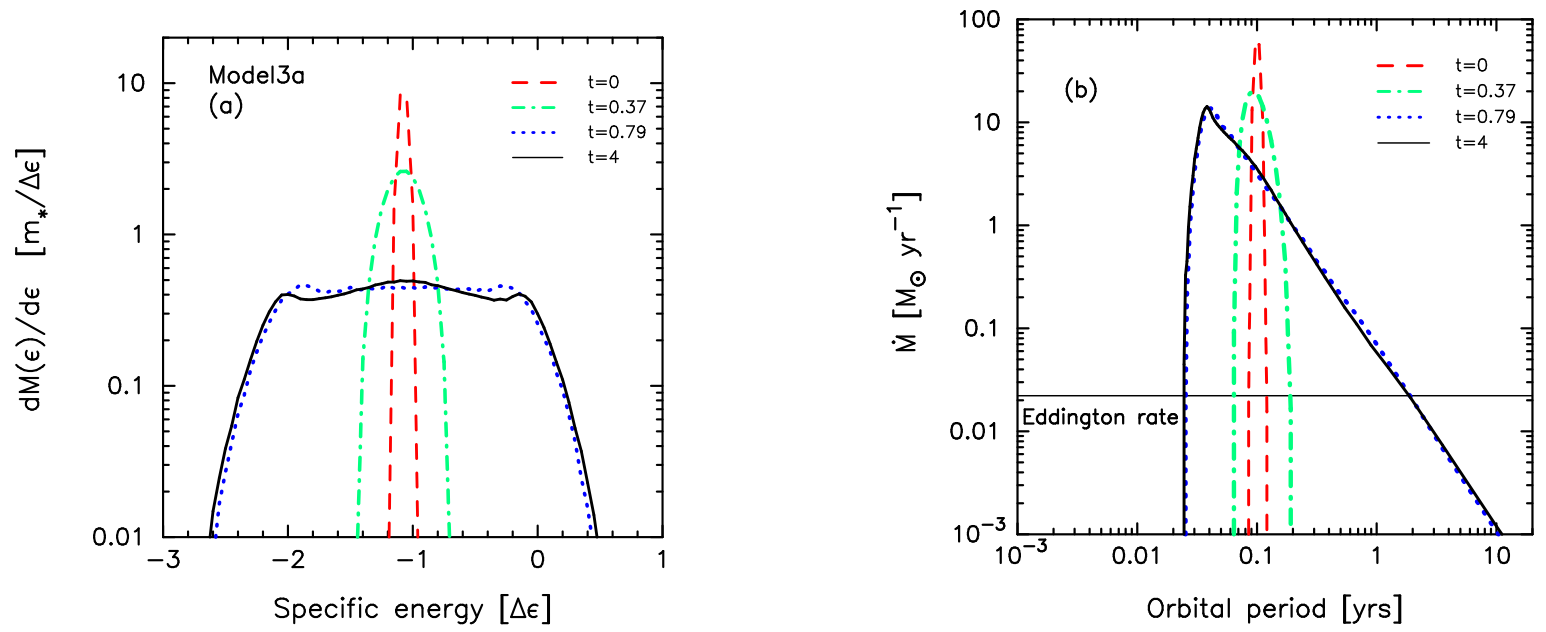

Figure 5.9.- : Same as Fig. 5.5, but for Model 3a. 

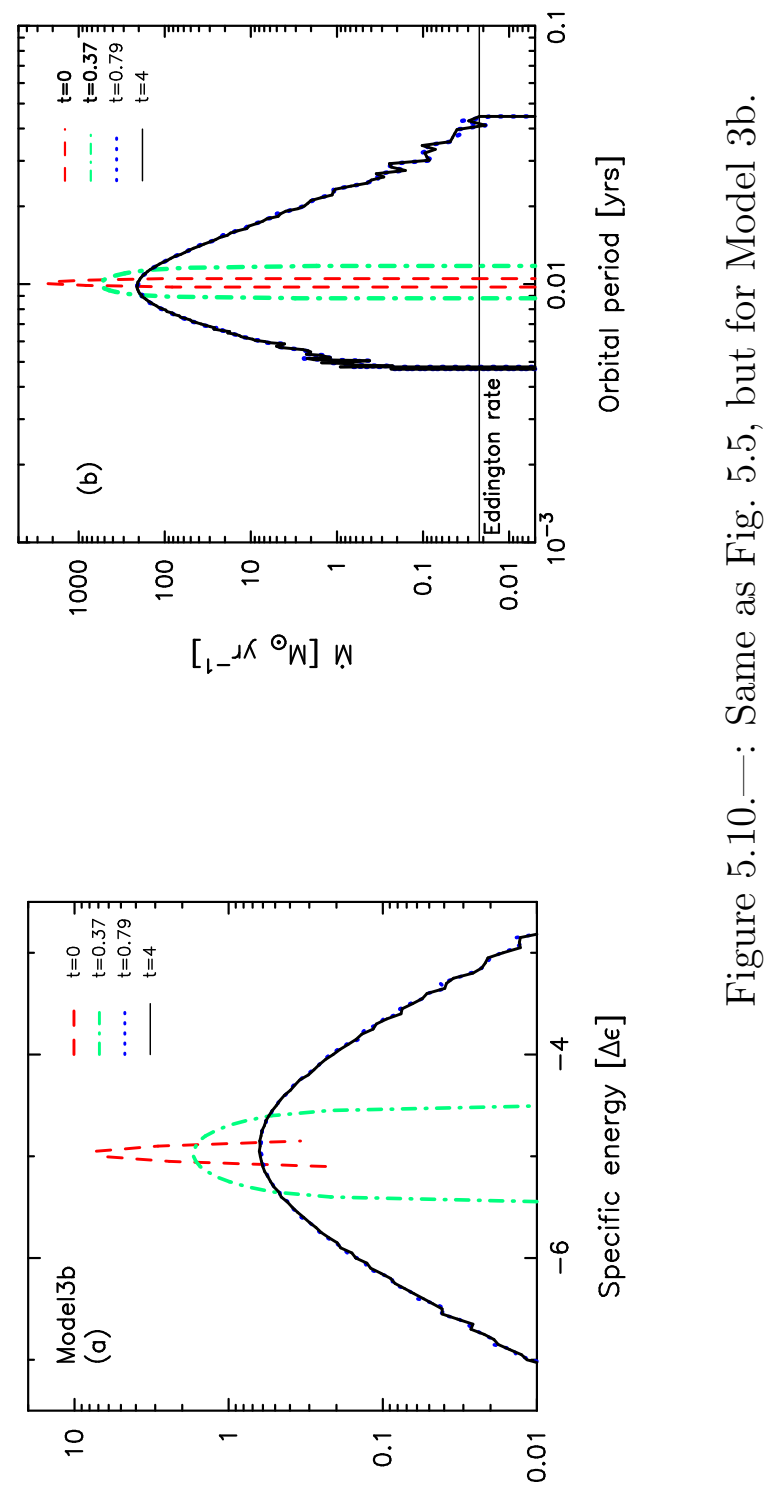

$\left[\ni \nabla /{ }^{*} \mathrm{~W}\right] \quad \ni \mathrm{эP} /(\ni) \mathrm{WP}$ 


\subsubsection{Accretion disk formation}

Here we describe how an accretion disk forms rapidly around the black hole in Model 2 a. The orbital angular momentum of a star passing inside the tidal disruption radius should be conserved before and after the tidal disruption, if there is no mechanism to redistribute the angular momentum. The orbits of the stellar debris can then be circularized by dissipation of orbital energy, primarily due to shocks from orbit crossing, which conserve orbital angular momentum. Debris semi-major axes will thus approach the circularization radius of the initial stellar orbit.

The specific energy and specific angular momentum of a test particle with the orbital parameters of Model 2a, moving under the pseudo-Newtonian potential, are given as $\epsilon_{\mathrm{tp}}=-0.497$ and $l_{\mathrm{tp}}=0.714$, respectively, by substituting $\left(r_{\mathrm{p}}, r_{\mathrm{a}}\right)=\left(0.2 r_{\mathrm{t}}, 1.8 r_{\mathrm{t}}\right)$ with $(e, \beta)=(0.8,5)$ into equations (5.8)-(5.10). Fig. 5.11 shows the evolution of an averaged specific energy, specific internal energy, and specific angular momentum of SPH particles in Model 2a. In panel (a), the solid line and the dashed line show the averaged specific energy of SPH particles and the specific energy of a test particle, respectively. The small inside panel shows the evolution of the averaged specific internal energy of SPH particles, which is dissipated and radiated away after $t=2.4 \Omega^{-1}$ due to the shocks from self-intersections of the debris orbits induced by relativistic precession. Since the internal energy contributes negligibly to the total energy budget, the specific energy approximately equals the specific binding energy. On the other hand, panel (b) shows the evolution of the averaged specific angular momentum of SPH particles normalized by $l_{\text {tp. }}$ Its marginal fluctuation is a numerical error with a magnitude of $0.15 \%$, indicating that the specific angular momentum is almost conserved. Assuming that the circularized 

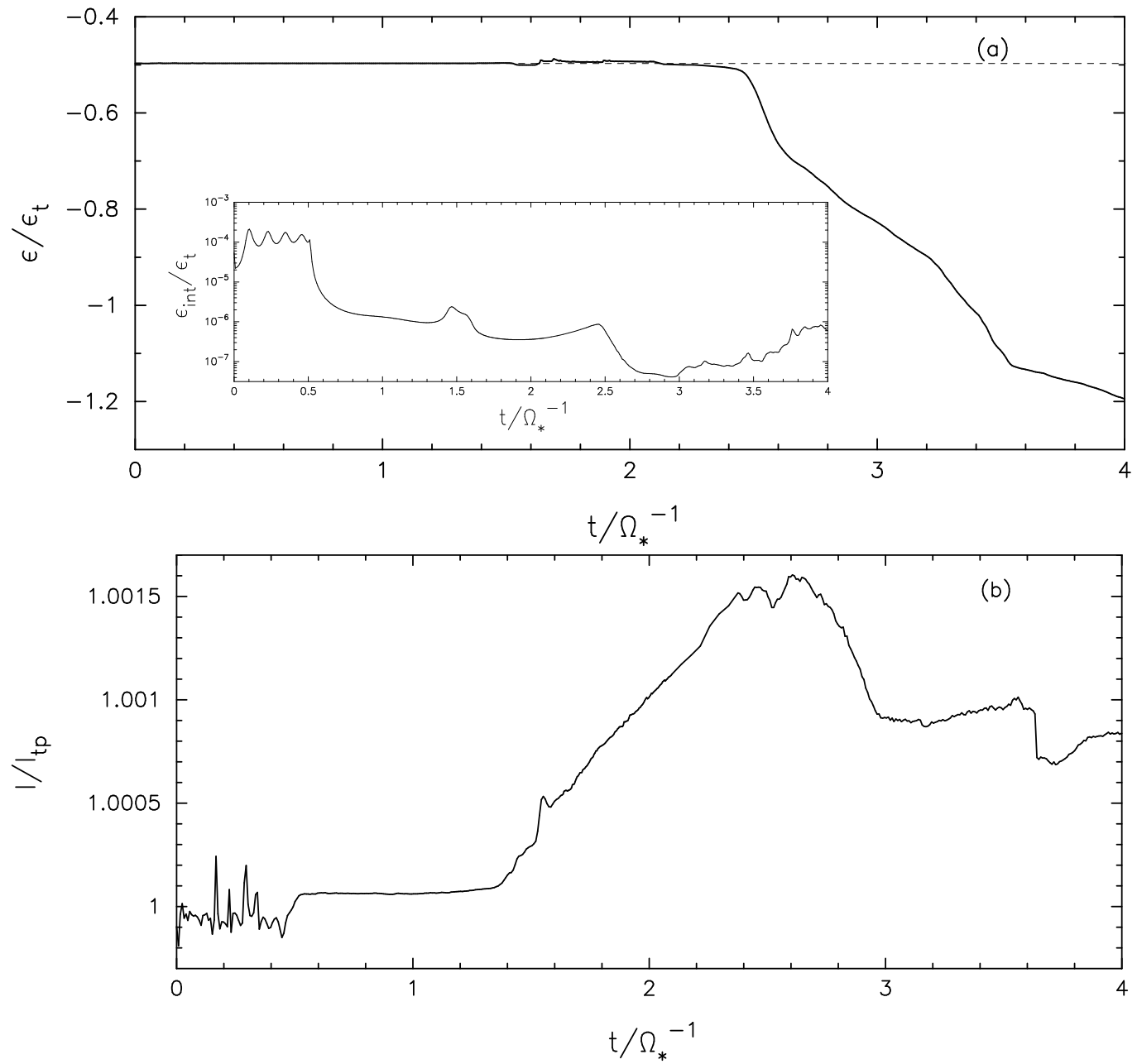

Figure 5.11.- : Evolution of the averaged specific energy and specific angular momentum of SPH particles in Model 2a. In panel (a), the solid line and dashed line represent the averaged specific energy of SPH particles and the specific energy of a test particle $\epsilon_{\mathrm{tp}}=-0.497$, respectively. The small inside panel shows the evolution of the averaged specific internal energy of SPH particles, $\epsilon_{\text {int }}$. It is normalized by $\epsilon_{\mathrm{t}}=G M / r_{\mathrm{t}}$. Panel (b) shows the averaged specific angular momentum of SPH particles normalized by $l_{\mathrm{tp}}=0.714$, which is the specific angular momentum of a test particle. 

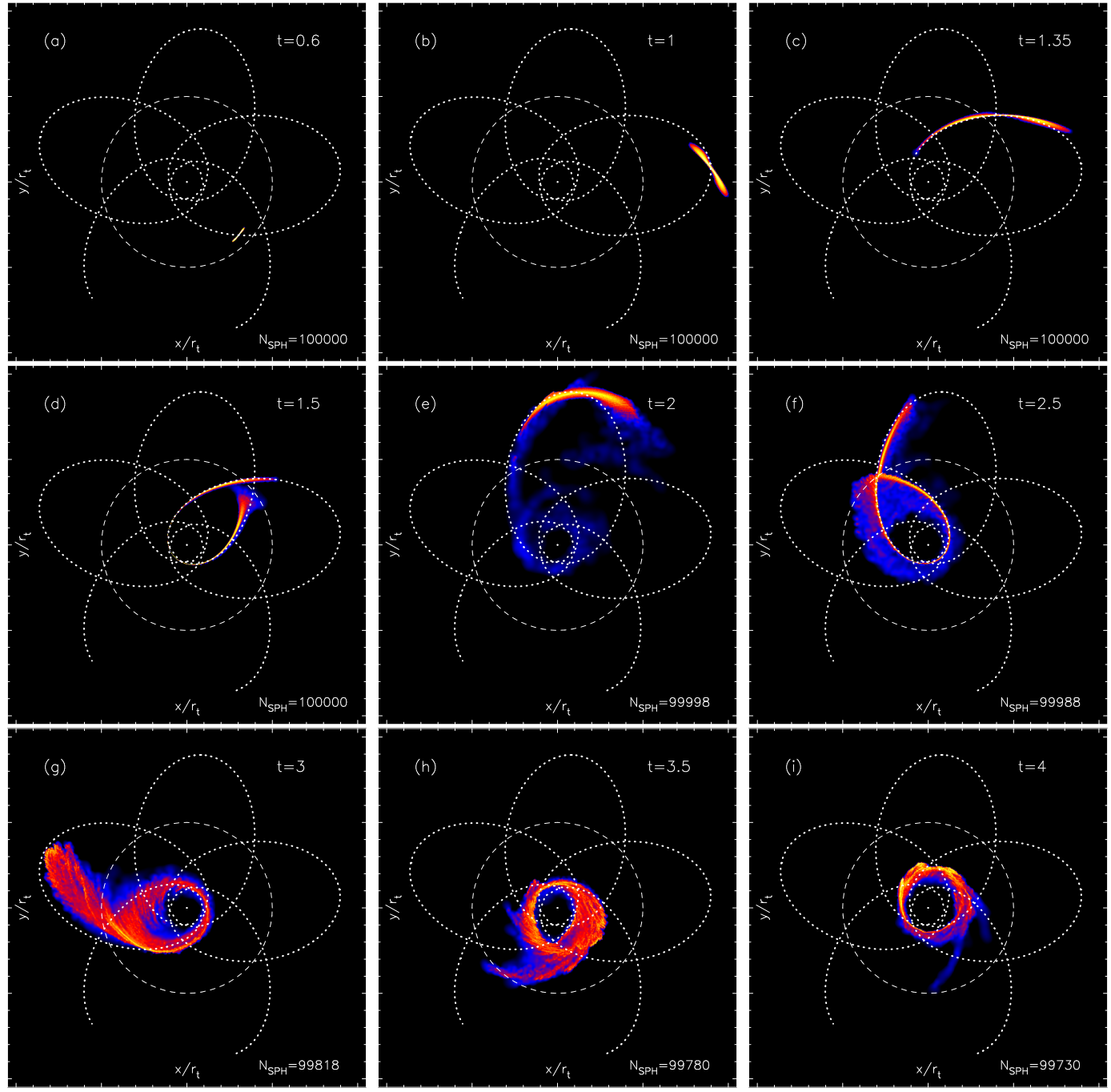

Figure 5.12.- : A sequence of snapshots of the tidal disruption process in Model 2a. They are from panel (a) to panel (i) in chronological order. Each panel shows a surface density projected on $x$-y plane in five orders of magnitude in a logarithmic scale for $0.6 \leq t \leq 4$, where $t$ is in units of $\Omega_{*}^{-1}$. The black hole is set at the origin. The run time is annotated at the top-right corner, while the number of $\mathrm{SPH}$ particles are indicated at the bottom-right corner. The dashed circle and dotted line indicate the tidal disruption radius and the orbits of a test particle moving under the pseudo-Newtonian potential given by equation (5.10), respectively. 
CHAPTER 5. ECCENTRIC TIDAL DISRUPTIONS
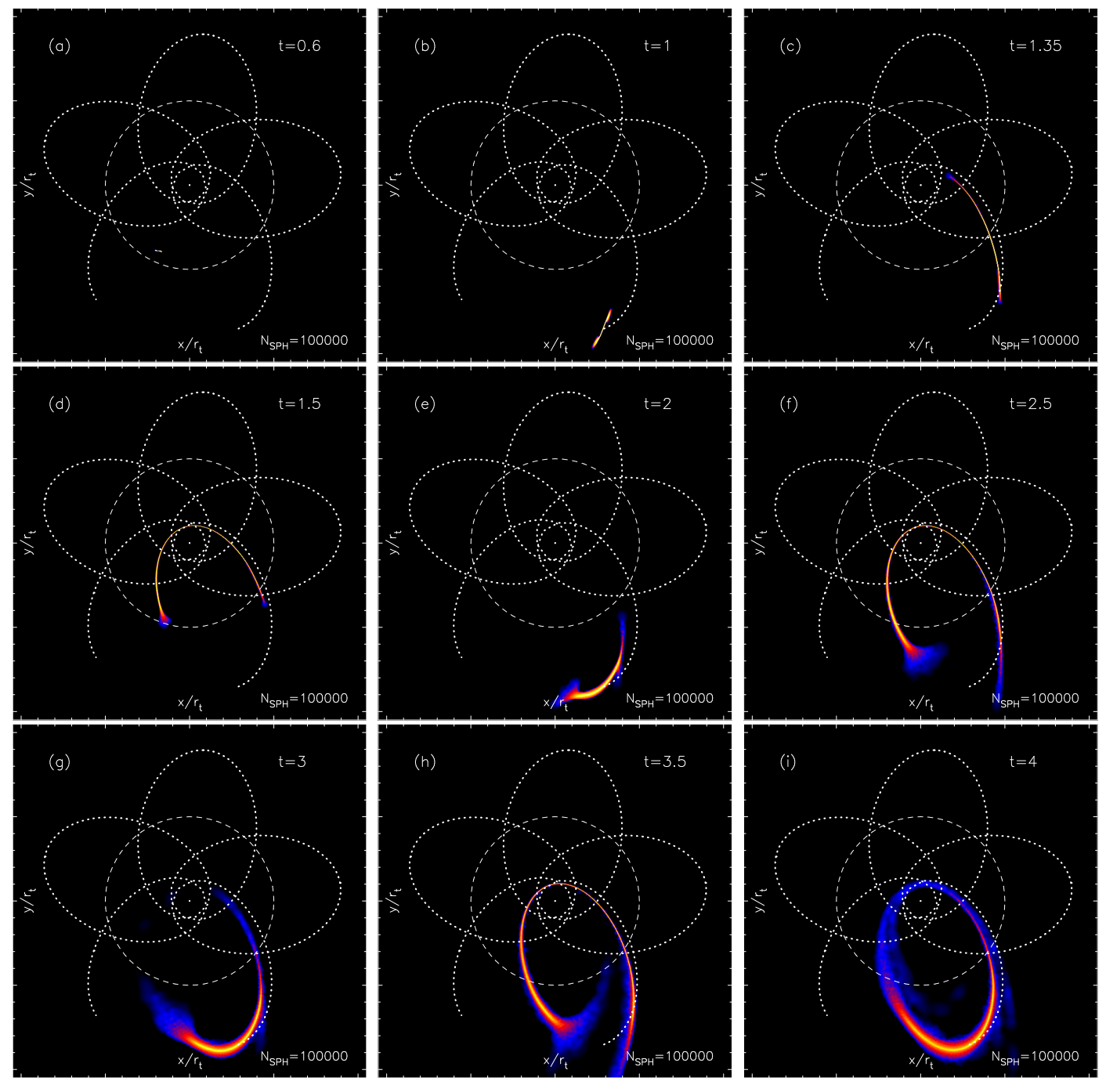

Figure 5.13.- : Same format as Model 2a but for Model 2b. 


\section{CHAPTER 5. ECCENTRIC TIDAL DISRUPTIONS}

disk has an axisymmetric Keplerian rotation, we can evaluate that the circularization radius $r_{\text {circ }}$ is $\approx 2.5 r_{\mathrm{p}}$.

Fig. 5.12 shows sequential snapshots of the surface density of stellar debris (projected on the $x-y$ plane in five orders of magnitude, in a logarithmic scale) for Model 2a. The central small point, dashed circle, and dotted line show the black hole, tidal disruption radius, and orbits of a test particle moving under the pseudo-Newtonian potential, respectively. The run time is noted at the top-right corner in units of $\Omega_{*}^{-1}$, while the number of SPH particles are indicated at the bottom-right corner. The star is tidally disrupted before it passes through the first pericenter at $t=0.5$. Afterward, the stellar debris expands along the orbit of the test particle as shown in panel (a) and (b). It is stretched towards the second pericenter as shown in panel (c). At the second pericenter, the stretched debris weakly intersects with the remnant of the debris in panel $(d)$ and then reaches the second apocenter while continuing to expand, in panel (e). When it reaches the third pericenter in panel (f), two stretched orbits clearly cross over. The orbital energy is significantly dissipated by the shock from orbit crossing between the two stretched debris streams. This is consistent with the decrease of specific energy in panel (a) of Fig. 5.11. The stellar debris is therefore rapidly circularized as shown in panels $(\mathrm{g})$ and $(\mathrm{h})$. Finally, in panel (i), a disk like structure is formed around the black hole sufficiently inside the tidal disruption radius. Note that the number of SPH particles are slightly reduced from panel (e) to panel (i), since a very small fraction of the total number of SPH particles is accreted onto the black hole. The trajectory of a SPH particle picked out of this simulation for $0 \leq t \lesssim 4$ is drawn in the solid line of

Fig. 5.3. The size of the accretion disk is in rough agreement with $r_{\text {circ }} \approx 2.5 r_{\mathrm{p}}$.

Although the pseudo-Newtonian potential does not accurately model the precession 


\section{CHAPTER 5. ECCENTRIC TIDAL DISRUPTIONS}

of near-circular orbits, we note that by the time the pseudo-Newtonian potential becomes significantly inaccurate, the debris streams have entered a regime of frequent orbit crossings, guaranteeing further rapid circularization. Furthermore, at a qualitative level the pseudo-Newtonian potential probably overestimates the circularization timescale, as it underestimates the true rate of relativistic precession for eccentric orbits. However, the pseudo-Newtonian potential still plays a crucial role in orbital circularization processes, because the motion of a test particle under the Newtonian potential take place in a closed

path and therefore causes no orbit crossing. In order to test this, we have performed the simulation for Model 2b, which has the same simulation parameters as Model 2a except that the Newtonian potential is adopted. Fig. 5.13 shows sequential snapshots of the surface density of stellar debris for Model 2b. It has the same format as Fig. 5.12. We note that there is no significant evidence for orbital crossings during the timescale of the simulation, since SPH particles orbit around the black hole on fixed eccentric orbits. This is also confirmed by the fact that the percentage change of specific orbital energy from $\mathrm{t}=0$ to $\mathrm{t}=4$ for Model $2 \mathrm{~b}$ is less than $0.4 \%$.

Fig. 5.14 shows the time-dependence of the number of SPH particles inside the tidal disruption radius $N_{\text {acc }}$ and its first derivative (the mass capture rate). After the tidal disruption of the star, its orbit passes through the first apocenter, going completely outside of the tidal disruption radius. The first peak of $N_{\text {acc }}$ in panel (a) comes when the debris streams pass from the first apocenter to the second apocenter via the second pericenter, and the stretched debris re-enters the tidal disruption radius. Part of it exits once more, but the fractional remaining part is still inside the tidal disruption radius. A sequence of these events can be seen in panels (c)-(e) of Fig. 5.12. The stretched debris returns again to the tidal disruption radius, moving toward the third pericenter. 


\section{CHAPTER 5. ECCENTRIC TIDAL DISRUPTIONS}

Afterwards, most of debris circularizes and remains inside of the tidal disruption radius.

Panel (b) of Fig. 5.14 shows the rate of mass being captured inside the tidal disruption radius. The first three peaks are formed as stellar debris passes in and out of the tidal disruption radius, while the final peak shows mass circularizing into the accretion disk around the black hole. The mass accretion rate is more than five orders of magnitude higher than the Eddington rate (see equation 5.17). This shows that the accretion flow is extremely supercritical in the case of moderately eccentric TDEs.

Figs. 5.15 and 5.16 show the evolution of differential mass distributions and corresponding mass fallback rates in Model 2a and Model 2b. The figure formats are the same as Figs. 5.5-5.10, except that solid (black) line shows the differential mass distribution at $t=1.35$ and corresponding mass fallback rate. The mass is distributed around $-50 \Delta \epsilon$ in Model $2 \mathrm{~b}$ as is expected, whereas the differential mass distribution of Model 2a slightly deviates from $-50 \Delta \epsilon$ to the positive direction because of corrections of the specific energy from the pseudo-Newtonian potential (see equations (5.8) and (5.10)). The resultant mass-fallback rate of Model 2a corresponds to that of Model 2b except for the shape of peak of mass fallback rate. This is consistent with our estimate of the mass fallback rate, $\sim 10^{4} M_{\odot} \mathrm{yr}^{-1}$, calculated by taking the ratio of the bound mass, $1 M_{\odot}$, to the fallback time $\Delta t$ for Models $2 \mathrm{a}$ and $2 \mathrm{~b}$ (see also equation (5.24)).

It is noted by definition that the second peak of mass capture rate in panel (b) of Fig. 5.14 corresponds to the mass fallback rate of Model 2a. It completely deviates from the canonical $t^{-5 / 3}$ law, since $t_{\max } / t_{\min }$ for Model 2a is estimated to be $\sim 3$ from equations (5.21) and (5.22). This is because the dependence of $t^{-5 / 3}$ on the mass fallback rate can be seen only when $t_{\max } \gg t_{\min }$. 
Since the viscous timescale measured at $r_{\text {circ }}$ is estimated to be $t_{\mathrm{vis}} \sim 3.5 \times$ $10^{3}\left(0.1 / \alpha_{\mathrm{SS}}\right) \Omega_{*}^{-1}$ where $\alpha_{\mathrm{SS}}$ is the Shakura-Sunyaev viscosity parameter, the accretion flow is clearly in a non-steady state. Here, we assume that the accretion disk is a geometrically thick : $r_{\text {circ }} / H \sim 1$, where $H$ is the disk scale height. Although the fate of the circularized debris is unclear because of much shorter simulation time than the viscous timescale, the super-Eddington accretion flow will likely drive a powerful outflow (Ohsuga et al. 2005) as it becomes radiation-pressure dominated. This may increase the optical luminosity of the flare by orders of magnitude (Strubbe \& Quataert 2009). It is even possible that a radiation-pressure supported envelope could be formed (Loeb \& Ulmer 1997). 


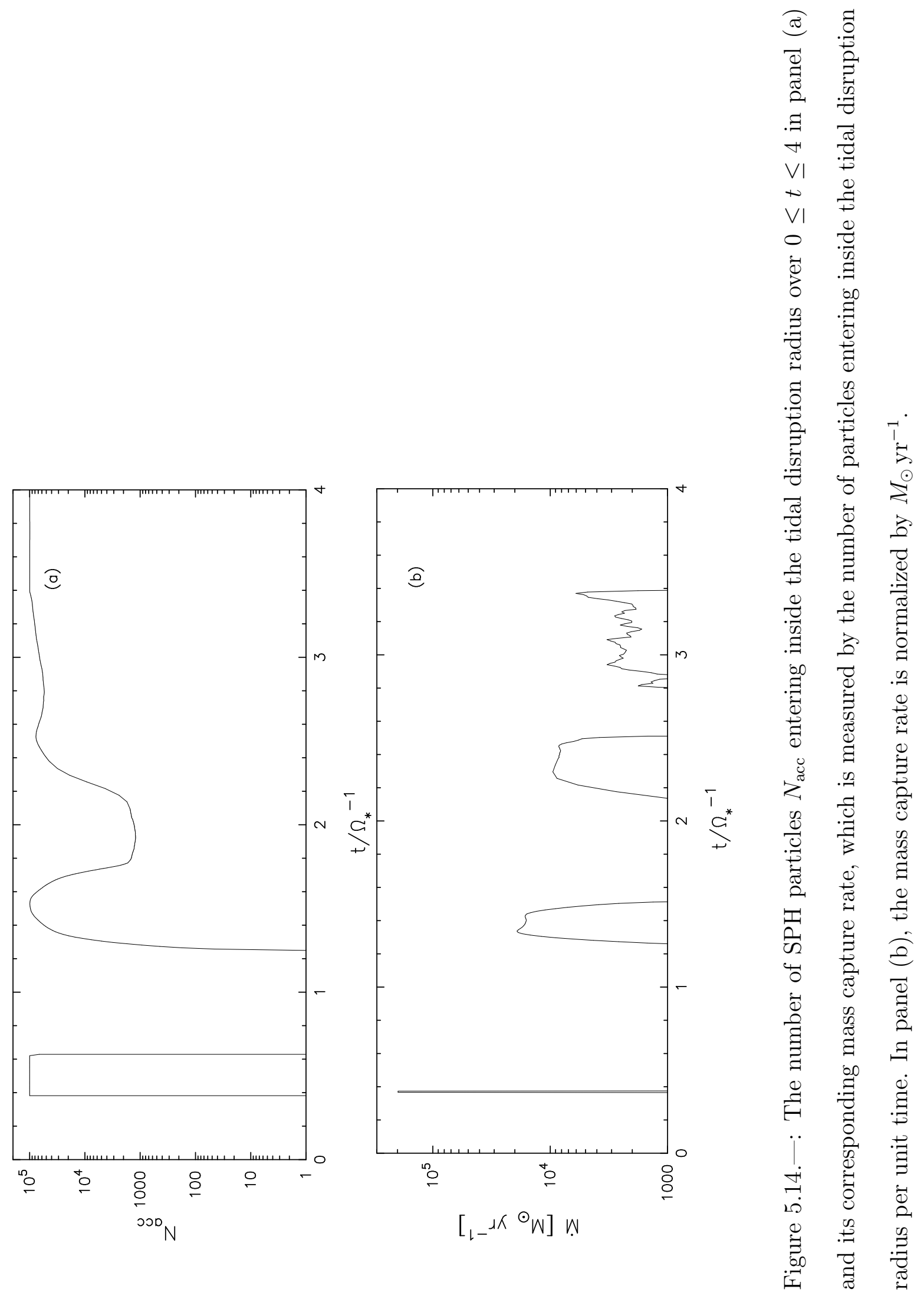




\subsection{Sources of Eccentric TDEs}

Although the canonical source for TDEs is two-body scattering at parsec scales, a variety of alternate mechanisms exist. In this section we briefly review these, and highlight those which could supply stars at eccentricities $e<e_{\text {crit }}$ to their central SMBH.

- Resonant relaxation: at small spatial scales close to a $\mathrm{SMBH}$, the approximately Keplerian potential causes stellar angular momentum to relax in a correlated, coherent way (as opposed to the uncorrelated changes from two-body relaxation). It has been suggested that the rapid changes in orbital eccentricity produced by resonant relaxation $(\mathrm{RR})$ may enhance the TDE rate at small scales (Rauch \& Ingalls 1998); however, recent work has indicated that the complicated interplay between RR and general relativistic precession gives rise to a "Schwarzschild barrier," which quenches RR for stars with small semi-major axis (Merritt et al. 2011). RR is therefore unlikely to produce TDEs with $e<e_{\text {crit }}$.

- Nuclear triaxiality: conversely, the triaxial, non-Keplerian potential of a surrounding star cluster results in fewer constants of motion for stellar orbits; in particular, angular momentum is not conserved and some stars can wander arbitrarily close to the SMBH, eventually being disrupted (Merritt \& Poon 2004). However, this effect only arises at large radii where the potential from the star cluster can induce a significant triaxial correction to the SMBH potential, and is therefore unlikely to produce low eccentricity TDEs.

- A nuclear stellar disk: there is a rotating stellar disk composed of young massive stars at the center of the Milky Way (Bartko et al. 2010). These stars orbit with 

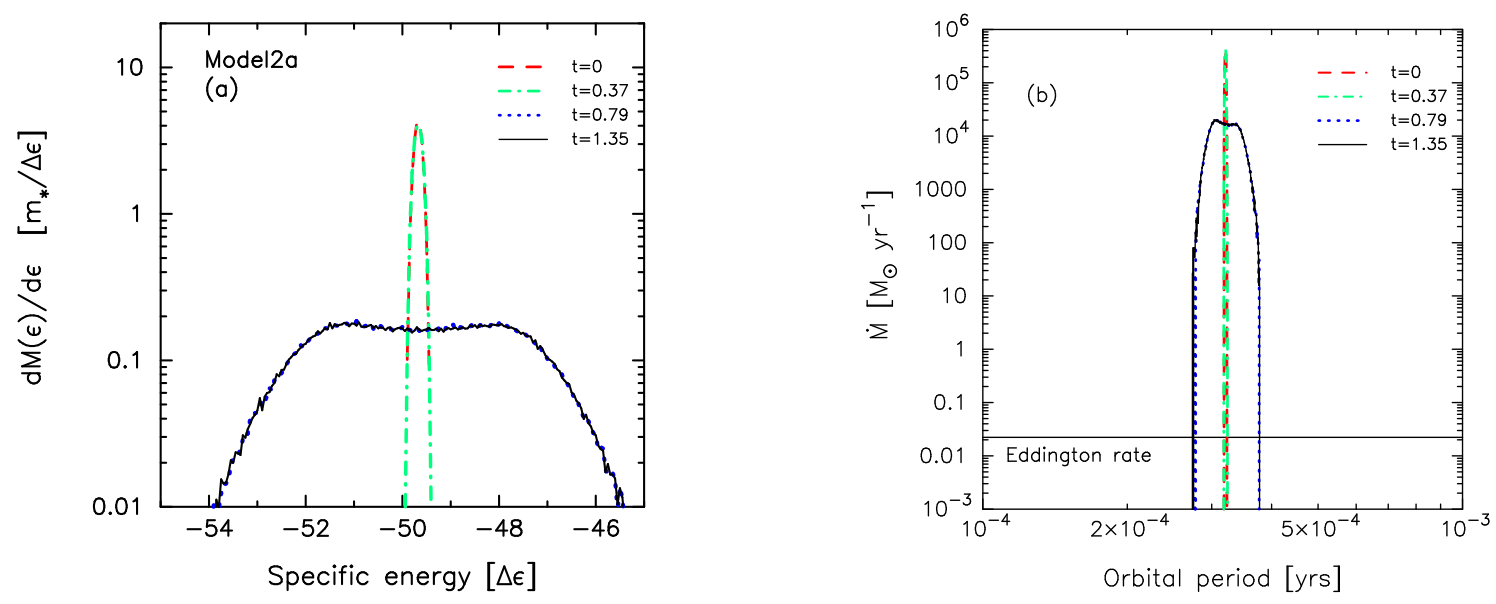

Figure 5.15.- : Same as Fig. 5.5, but for Model 2a.
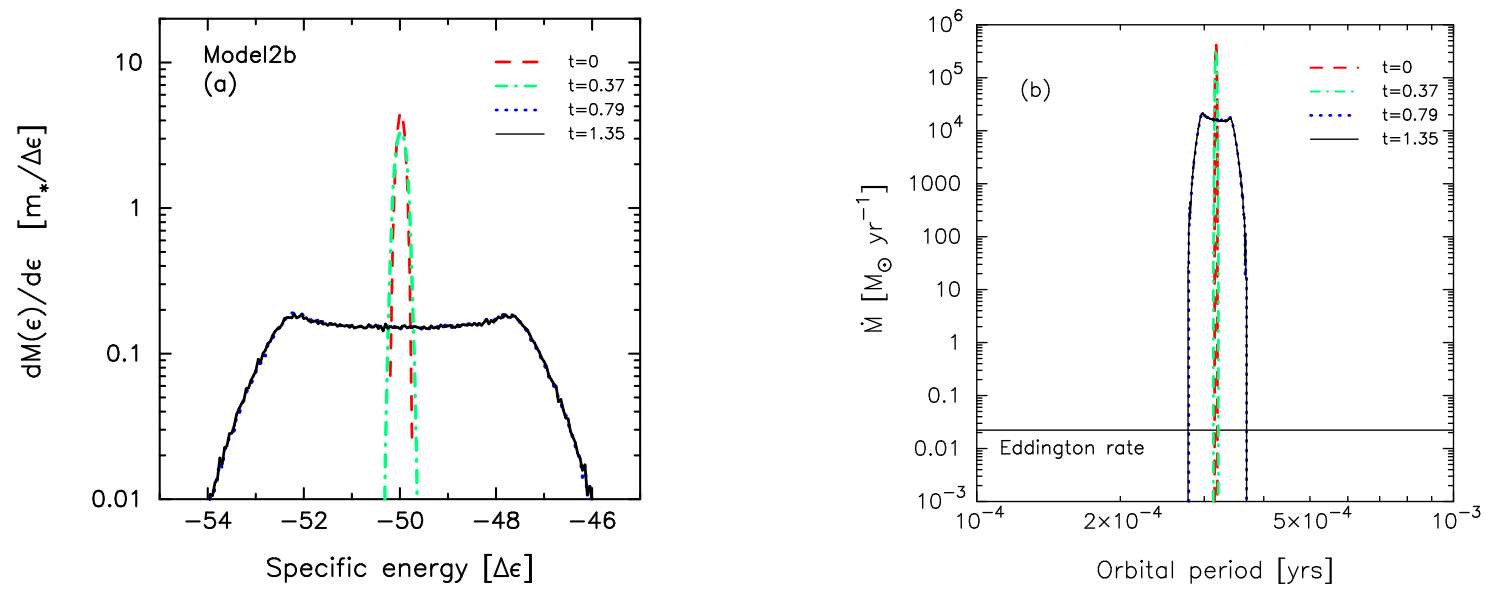

Figure 5.16.-: Same as Fig. 5.5, but for Model 2b. 
moderate eccentricity around the supermassive black hole SgrA*. The nonresonant relaxation timescale drops as a star cluster becomes flattened and disk-like, due to greater encounter rates between stars; simple estimates of the two-body eccentricity relaxation timescale in the Milky Way's stellar disk $\left(\sim 5000 M_{\odot}\right)$ find values $t_{\mathrm{rlx}} \sim 4 \times 10^{8}$ yrs (Kocsis \& Tremaine 2011). Assuming a typical stellar mass of $10 M_{\odot}$ gives a disk star TDE rate of $\sim 10^{-6} \mathrm{yr}^{-1}$, implying that if analogous disks are common in other galaxies, they could contribute nontrivially to the total TDE rate. However, in order to produce TDEs with $e<e_{\text {crit }}$, disk stars would need to be scattered in from $\sim$ mpc scales, implying that more compact disks than the Milky Way's are needed to produce significantly eccentric TDEs.

- Binary SMBHs: A binary SMBH system will, for a period of $\sim 10^{5}$ yr to $\sim 10^{6} \mathrm{yr}$, see a TDE rate enhancement up to $10^{-1} \mathrm{yr}^{-1}$ from a combination of chaotic orbital evolution and Kozai cycles (Ivanov et al. 2005; Chen et al. 2009). Recent work suggests that chaotic orbits in particular are the dominant contribution to the rate enhancement (Chen et al. 2011; Wegg \& Nate Bode 2011). Most of the stellar flux originates from within one order of magnitude of the semimajor axis of the binary; since the inspiral of binary SMBHs stalls when the lower-mass secondary reaches a radius inside of which is contained its own mass in stars, a low binary mass ratio $q$ will produce a flux of less eccentric TDEs. In particular, Fig. 17 of Chen et al. (2011) indicates that for $q=1 / 81$ and a primary black hole mass of $10^{7} M_{\odot}$, some stellar flux into the primary's loss cone originates from spatial scales $\leq 10^{15} \mathrm{~cm}$, which implies $e<e_{\text {crit }}$ even for $\beta=1$ events. However, the chaotic orbits which dominate the rate enhancement produce TDEs sampling a wide range of $\beta$ (Merritt \& Poon 2004), so the situation is even 
more favorable. Binary SMBHs on a eccentric orbit appear to produce even more TDEs from small apocenters. The "hardening radius" (the orbital radius within which the stellar cusp of the primary, larger SMBH contains the mass in stars of the secondary, smaller SMBH) effectively sets the scale at which stellar disruption rates are enhanced; to produce eccentric TDEs requires relatively low mass ratios $(<1 / 50)$ which could in some cases be characterized as SMBH-IMBH inspirals. Because binary SMBHs are expected to provide up to $10 \%$ of the total TDE rate (Wegg \& Nate Bode 2011), it is important to note that a subset of these events may deviate from canonical "parabolic" light curves.

- SMBH recoil: the gravitational wave recoil accompanying a SMBH merger will scramble the orbits of surrounding stars and partially refill the kicked merger remnant's loss cone, briefly increasing the TDE rate. If one considers the bulk of the stellar population surrounding the merging binary black holes, the excavation of a "binary loss cone" results in too few stars at small separation for the resulting burst of TDEs to involve any events with $e<e_{\text {crit }}$ (Stone \& Loeb 2011). However, at low binary mass ratios, it is possible for mean motion resonances to migrate stars to small semimajor axes during the binary inspiral (Schnittman 2010; Seto \& Muto 2010, 2011); any TDEs produced from this stellar subpopulation would have $e<e_{\text {crit }}$, and would be described by this paper.

- Binary separation: Recent theoretical studies on rates of tidal separation of binary stars by SMBHs suggest that a significant fraction of tidal disruption flares may occur from stars approaching the black hole from subparsec scales (Amaro-Seoane et al. 2012a; Bromley et al. 2012). When a binary star passes sufficiently close to the black hole to be tidally separated (without immediate 


\section{CHAPTER 5. ECCENTRIC TIDAL DISRUPTIONS}

disruption of either component), one star becomes tightly bound to the black hole while the other is flung away at a high speed. The subsequent orbital evolution of the bound star will represent a competition between gravitational radiation and stellar relaxation processes. If gravitational radiation dominates, the orbit will circularize and spiral in, likely leading to a phase of steady mass transfer that is unlike the eccentric and violent disruptions which we have simulated in this paper. On the other hand, if stellar relaxational processes dominate, the star can eventually diffuse into the loss cone with a nonzero eccentricity that is still significantly smaller than that expected for TDEs generated by two-body relaxation at parsec scales. For a bound star with $r_{\mathrm{p}}>r_{\mathrm{t}}$ and semimajor axis $a$, living in a stellar cusp with density profile $\rho(r) \propto r^{-b}$, the eccentricity dividing gravitational-wave-dominated evolution from relaxation-dominated evolution is (Amaro-Seoane et al. 2012a)

$$
\begin{aligned}
e_{\mathrm{gw}} \approx 1- & 0.016 \times\left(8 \times 10^{-4}\right)^{(2 b-3) / 5}(3-b)^{2 / 5} \\
& \times\left(\frac{M_{\mathrm{BH}}}{10^{7} M_{\odot}}\right)^{(8-b) / 5}\left(\frac{a}{1000 \mathrm{AU}}\right)^{(2 b-11) / 5}
\end{aligned}
$$

In practice, requiring $e_{\mathrm{gw}}>e_{\text {crit }}$ for $a<r_{\mathrm{t}} /\left(1-e_{\text {crit }}\right)$ is only possible for steep stellar cusps $b \approx 2$ and very low mass $\operatorname{SMBHs}\left(M_{\mathrm{BH}} \leq 10^{5.5} M_{\odot}\right)$. A further complication is the presence of the Schwarzschild barrier, which may prevent stars with long gravitational wave inspiral timescales from entering the loss cone. Stars bound to a SMBH from binary separation events can become TDEs with lower eccentricity than in the canonical scenario, but equation (5.25) implies their eccentricity will still tend to be greater than $e_{\text {crit }}$. On the other hand, equation (5.25) was derived assuming the Fokker-Planck diffusion limit for two-body relaxation, and anomalous diffusion from strong scattering events (Bar-Or et al. 2013) may allow violent tidal 


\section{CHAPTER 5. ECCENTRIC TIDAL DISRUPTIONS}

disruption of separated binaries even from eccentricities $e<e_{\mathrm{gw}}$.

Of the possibilities we have considered, two stand out as particularly promising: TDEs generated as binary SMBHs harden and stall, and TDEs generated during the coalescence of binary SMBHs with a low mass ratio by stars that were brought inward via mean motion resonances. The first possibility may account for a significant fraction of the total TDE rate; the second possibility will be much less intrinsically common, but could serve as an electromagnetic counterpart to an eLISA/NGO²-band gravitational wave signal. It is also possible that strong scatterings could lead to eccentric TDEs from the bound stars produced by tidal separation of stellar binaries. Other possible enhancements to theoretical TDE rates, such as perturbations from infalling giant molecular clouds, likely occur at too large of a spatial scale to create eccentric TDEs.

\subsection{Summary \& Discussion}

We have carried out numerical simulations of mass fallback and accretion processes around a SMBH. Specifically, we have examined the tidal disruption of a star on a bound orbit, considering relativistic effects with a pseudo-Newtonian potential. Using a polytropic gas sphere as our initial conditions, we have considered both parabolic orbits $(e=1.0)$ and eccentric ones $(e=0.8$ and 0.98$)$, varying the penetration factor $\beta$ as well.

We have found that a non-steady, non-axisymmetric accretion disk is formed around the black hole in the case of $\mathrm{e}=0.8$ and $\beta=5$. The formation of an accretion disk occurs as follows: a segment of the stellar debris returning to pericenter, and a different one

\footnotetext{
${ }^{2}$ http://www.elisa-ngo.org/
} 


\section{CHAPTER 5. ECCENTRIC TIDAL DISRUPTIONS}

exiting pericenter, intersect each other due to relativistic precession. The orbital energy is then dissipated by shocks due to orbit crossing. Since the orbital angular momentum of the stellar debris is conserved before and after the tidal disruption, the debris orbits are rapidly circularized during a few orbit crossings. This shows that the initial size of the accretion disk is only determined by the orbital angular momentum of the initial stellar orbit. In our simulations, the circularization radius is estimated as $r_{\text {circ }} \approx 2.5 r_{\mathrm{p}}$ where $r_{\mathrm{p}}$ is the pericenter distance. Furthermore, the expected accretion rate is extremely super-Eddington.

As shown in the final peak of Fig. 5.14, panel (b), the mass accretion rate exhibits variability during the formation of the accretion disk around the black hole. After some viscous evolution of the accretion disk, however, the variation of the mass accretion rate would settle into that of the mass fallback rate as shown in panel (b) of Fig. 5.15. Observations of TDE light curves roughly following $t^{-5 / 3}$ power laws may suggest that the more common parabolic disruption scenario can circularize efficiently as well, perhaps through GR precession as in the eccentric TDE case. This will need to be demonstrated although it takes much computational time.

The striking difference between moderate-eccentricity simulations with and without relativistic precession highlights the importance of general relativistic effects for debris circularization. Specifically, very little energy dissipation was seen in Model $2 \mathrm{~b}$ (Newtonian) of Table 1, while its pseudo-Newtonian equivalent, Model 2a, saw rapid accretion disk formation. For reasons of computational cost, we did not follow the longer timescales required for debris circularization in other models, but in future work we hope to investigate if these results generalize to higher eccentricity and parabolic TDEs. Sufficiently rapid SMBH spin could delay disk formation, as Lense-Thirring 


\section{CHAPTER 5. ECCENTRIC TIDAL DISRUPTIONS}

torques will precess the orbital planes of individual debris streams and limit or prevent orbit-crossings. If a disk is able to eventually form, its luminosity will be periodically modulated by Lense-Thirring precession (e.g., Stone \& Loeb 2012a). Even considering this complication, the energy dissipation process is crucial for determining the formation and structure of an accretion disk. In these exploratory simulations, we have adopted the polytropic equation of state instead of solving an energy equation. In a subsequent paper, we will study the detailed disk formation and structure by solving more realistic energy equations with and without a radiative cooling term.

Circularization has rarely been seen in past simulations of main sequence stars on parabolic orbits being disrupted by massive black holes, due to the difficulty in following orbits over their full dynamic range. While we have avoided this difficulty by simulating eccentric center of mass trajectories, there are two other examples of circularizing TDE debris in the literature (Ayal et al. 2000; Ramirez-Ruiz \& Rosswog 2009).

In Ramirez-Ruiz \& Rosswog (2009), debris circularization is seen in Newtonian simulations of stars on parabolic orbits being disrupted by $10^{3} M_{\odot}$ intermediate mass black holes. In this case, circularization is driven by vertical shocks at pericenter, which serves as a "nozzle" in vertically compressing returning gas. This redistributes orbital elements of the gas and act as a source of "effective precession," causing some gas leaving its second pericenter passage to impact gas returning from its first apocenter passage. Whether or not this nozzle-driven circularization scales up to $e=1$ orbits around SMBHs is unclear.

The SPH simulations of Ayal et al. (2000) also appear to capture nozzle-driven circularization in $e=1, M_{\mathrm{BH}}=10^{6} M_{\odot}$ TDEs. However, the reliability of these results 


\section{CHAPTER 5. ECCENTRIC TIDAL DISRUPTIONS}

may be limited by the low particle resolution of these simulations (only $5000 \mathrm{SPH}$ particles), and the particle-splitting algorithm employed to address resolution issues, since the vertical compression of the star will be sensitive to spurious changes in stream geometry. If vertical compression at pericenter is under-resolved, the net effect will be unphysical velocity perturbations in the orbital plane (Guillochon et al. 2009; Stone et al. 2012b), correspondingly unphysical apsidal precession, and unphysical circularization.

Eccentric TDEs are a subpopulation of all TDEs, but a potentially interesting one. The two most promising means of producing them are in the dynamical friction stage of binary SMBHs, and immediately following gravitational wave-driven black hole coalescence. Both of these are especially interesting subsets of TDEs: the former occurs during a time of greatly enhanced TDE rates, when it may be possible for a single galaxy to produce multiple TDEs in $\sim 10 \mathrm{yr}$, while the latter would serve as a delayed electromagnetic counterpart to a low-frequency gravitational wave signal. It is also possible that tidal separation of binary stars could produce TDEs of interestingly low-to-moderate eccentricity, if a large population of $M_{\mathrm{BH}} \leq 10^{5.5} M_{\odot}$ SMBHs reside in steep stellar cusps. Eccentric TDEs are also of interest because of the relatively short delay time between disruption and disk formation, allowing numerical simulations to bypass costly apocenter passages and directly approach open problems in debris circularization. The pseudo-Newtonian potential proposed by Wegg (2012) is, however, not applicable for the very low eccentricities of fully circularized gas because it was derived with the assumption of small binding energy. Therefore, we hope to employ post-Newtonian corrections in a subsequent paper.

The ongoing tidal disruption of a large gas cloud (G2) falling into the center of the Milky Way galaxy is another scenario within the "eccentric tidal disruption" 


\section{CHAPTER 5. ECCENTRIC TIDAL DISRUPTIONS}

regime. When discovered, the G2 gas cloud was estimated to have a mass $\sim 3 M_{\oplus}$, and to possess a radius $\sim 1.8 \times 10^{15} \mathrm{~cm}$ (Gillessen et al. 2012). More recent observations have resulted in a finer determination of the cloud's orbital parameters; it will soon reach its pericenter distance of $2.8 \times 10^{15} \mathrm{~cm}$, on an orbit with an eccentricity of 0.966 (Gillessen et al. 2013). This value of eccentricity is far less than the critical eccentricity for the cloud, and consequently all the debris will remain bound to SgrA*. Using equation (5.24), we find a characteristic mass fallback rate of $6.7 \times 10^{8} M_{\odot} \mathrm{yr}^{-1}$. This rate is likely an underestimate of the true mass fallback rate, which may be enhanced dramatically by ram pressure stripping near pericenter (Burkert et al. 2012; Schartmann et al. 2012; Anninos et al. 2012). Assuming immediate accretion of the returning matter would give a bolometric luminosity of $\sim 4 \times 10^{38} \mathrm{erg} / \mathrm{s}$ with an assumption that the mass-to-energy conversion efficiency is 0.1 . This would be several orders of magnitude brighter than the observed X-ray luminosity of $10^{33-34} \mathrm{erg} / \mathrm{s}$, detectable with the current X-ray satellites such as Chandra, Suzaku, and NuSTAR. However, the rate of matter fallback to pericenter will not necessarily equal the rate of accretion onto the black hole. The viscous timescale at pericenter is $t_{\text {visc }}=\left(\alpha_{\mathrm{SS}} \Omega_{\mathrm{p}}\right)^{-1}\left(H / r_{\mathrm{p}}\right)^{-2}$, where $\Omega_{\mathrm{p}}$ is the orbital frequency at the pericenter distance $r_{\mathrm{p}}$, and $H$ is the disk scale height at that radius. For the latest calibration of the orbital pericenter, and the approximate values $\alpha_{\mathrm{SS}}=0.1$, $H / r_{\mathrm{p}}=0.5, t_{\mathrm{visc}} \approx 7 \mathrm{yrs}$, long enough to significantly reduce the flare luminosity. It is also plausible that the accretion of G2 will proceed with radiative efficiency significantly under 0.1 (Mościbrodzka et al. 2012; Anninos et al. 2012), leading to a more modest increase in luminosity.

An additional source of luminosity during the tidal disruption of G2 could arise from tidal compression of the cloud in the direction perpendicular to its orbital plane. 


\section{CHAPTER 5. ECCENTRIC TIDAL DISRUPTIONS}

Although most hydrodynamical simulations of the disruption have been two-dimensional, some three-dimensional work has found significant (factors $\sim 100$ ) levels of vertical compression and density enhancement, and correspondingly large increases in $\operatorname{Br} \gamma$ line luminosity (Saitoh et al. 2012). The lack of change in observed $\operatorname{Br} \gamma$ luminosity (Gillessen et al. 2013) indicates that there should be room for improvement on the simple initial conditions of Saitoh et al. (2012) (i.e. a hydrostatic sphere, initialized in 1995). Although a detailed analysis of G2's tidal deformation is beyond the scope of this paper, we note that strong density enhancements from tidal compression are generic to the tidal disruptions of bodies initially in hydrostatic equilibrium (Stone et al. 2012b). If G2's origin is in a tidally disrupting proto-planetary disk (Murray-Clay \& Loeb 2012), the gravity of the central star could dominate the central regions and alter the tidal compression process; it is also possible that a shell-like configuration for the cloud (Schartmann et al. 2012) would avoid strong density enhancements until the upper and lower portions of the shell collide near pericenter.

As we have stressed in this paper, mass accretion following tidal disruption is mediated by circularization processes. However, the dramatic GR precession seen in our simulations is unlikely to be relevant for G2, as its pericenter is at a distance of 2200 Schwarzschild radii. Since the Galactic Center environment includes complex features such as an atmosphere of diffuse hot gas and warped stellar disks composed of young stars, dynamical interactions could dissipate the orbital energy and angular momentum of the G2 cloud and bring it closer to SgrA*. The most important interaction is likely to be ram pressure stripping shortly after pericenter passage, which has been seen to cause rapid circularization in multiple hydrodynamical simulations. Further observational and theoretical efforts will be necessary to clarify the future accretion of G2 onto SgrA*, 


\section{CHAPTER 5. ECCENTRIC TIDAL DISRUPTIONS}

which will differ in many important ways (ram pressure stripping, lack of GR precession, uncertain initial conditions) from the tidal disruption of a star on a comparably eccentric orbit.

For eccentric stellar orbits, there is a critical value of the orbital eccentricity below which all stellar debris is bounded to the black hole. It can be seen from our simulations that the critical eccentricity is slightly lower than expected from our analytical predictions. This might be because of the effects of stellar structure on the tidal disruption. There are three important implications for lightcurves of eccentric TDEs:

- The mass fallback rate will not asymptote to $\dot{M} \propto t^{-5 / 3}$ at late times but be finite with cut-off time $t=t_{\max }$.

- The lack of unbound debris will eliminate observational signatures associated with emission lines (e.g., Strubbe \& Quataert 2009).

- A larger amount of mass will return to pericenter in a much shorter time than in the standard parabolic picture, considerably increasing the ratio of $\dot{M} / M_{\mathrm{Edd}}$ for the subsequent flare.

Even for eccentricities $e>e_{\text {crit }}$, the center of the differential mass distribution will shift in a negative direction, providing weaker versions of the above effects. These signatures should be searched for when large samples of TDE candidates from next generation optical transient surveys such as the Large Synoptic Survey Telescope ${ }^{3}$ and

\footnotetext{
${ }^{3}$ http://www.lsst.org/lsst/
} 
CHAPTER 5. ECCENTRIC TIDAL DISRUPTIONS

next generation all-sky X-ray surveys such as extended Roentgen Survey with an Imaging Telescope Array ${ }^{4}$ become available.

${ }^{4}$ http://www.mpe.mpg.de/eROSITA 


\title{
Chapter 6
}

\section{Observing Lense-Thirring Precession in Tidal Disruption Flares}

N. Stone \& A. Loeb Physical Review Letters, Vol. 108, id. 061302, 2012

\begin{abstract}
When a star is tidally disrupted by a supermassive black hole (SMBH), the streams of liberated gas form an accretion disk after their return to pericenter. We demonstrate that Lense-Thirring precession in the spacetime around a rotating SMBH can produce significant time evolution of the disk angular momentum vector, due to both the periodic precession of the disk and the nonperiodic, differential precession of the bound debris streams. Jet precession and periodic modulation of disk luminosity are possible consequences. The persistence of the jetted X-ray emission in the Swift J164449.3+573451 flare suggests that the jet axis was aligned with the spin axis of the
\end{abstract}


SMBH during this event.

\subsection{Introduction}

The tidal disruption of a star by a supermassive black hole offers a unique opportunity to probe the nuclei of otherwise quiescent galaxies. However, the small number of candidate tidal disruption events (TDEs) makes it difficult to resolve theoretical uncertainties concerning their rates (Donley et al. 2002; Wang \& Merritt 2004; Merritt \& Poon 2004; Perets et al. 2007; Gezari et al. 2008; van Velzen et al. 2011b), super-Eddington accretion phase (Loeb \& Ulmer 1997; Strubbe \& Quataert 2009, 2011), and the period during which dissipation in shocks allows an accretion disk to form (Kochanek 1994; Ulmer 1999).

An additional outstanding question about TDEs is whether or not they produce jets, as observed in many other accreting black hole systems. The past year has seen both the first theoretical models for TDE-associated jets (Giannios \& Metzger 2011; van Velzen et al. 2011a) and the discovery by the Swift satellite of an intense, transient gamma- and X-ray flare from a galactic nucleus at $z \approx 0.35$ (Levan et al. 2011). This flare has been explained by multiple authors (Zauderer et al. 2011; Bloom et al. 2011; Burrows et al. 2011) as jet emission from a TDE aligned with our line of sight, although alternate hypotheses exist (Quataert \& Kasen 2012). A second possible TDE-associated jet was also recently observed (Cenko et al. 2012b).

If such jet emission is common, then TDEs provide a unique probe of the physics of accretion and jet production in the vicinity of distant black holes' horizons. Specifically, 
CHAPTER 6. DISK PRECESSION IN TDES

it is unknown at present whether jets will align with the black hole spin vector, the disk angular momentum vector, or some other component of the magnetic field geometry (Fragile 2008). In most black hole accretion environments these directions are parallel, but the transient disk of a TDE will generally have some tilt with respect to the SMBH equatorial plane. In this Letter we demonstrate that if jets from tilted TDE accretion disks align with the disk normal vector, they will generally be expected to precess, often by observable amounts. Even absent the existence of a disk-aligned jet, or any jet at all, general relativistic (GR) effects will precess TDE disks with potentially observable consequences.

\subsection{Spin Evolution of a Tilted Disk}

Stars of mass $M_{*}$ and radius $R_{*}$ that pass within a radius

$$
R_{\mathrm{t}}=R_{*}\left(M_{\mathrm{BH}} / M_{*}\right)^{1 / 3}
$$

of a black hole of mass $M_{\mathrm{BH}}$ will be tidally disrupted, with half their mass immediately unbound from the black hole (Rees 1988). For black holes of mass $M_{\mathrm{BH}} \gtrsim 10^{8} M_{\odot}$, the tidal radius $R_{\mathrm{t}}$ is inside the Schwarzschild radius $R_{\mathrm{S}}$ and stars are swallowed whole rather than disrupted. The bound debris rapidly expands and cools so that its pressure is negligible and the approximation of geodesic motion is accurate (Kochanek 1994). The most tightly bound debris stream of a star disrupted at radius $R_{\mathrm{P}}$ returns in a time

$$
t_{\text {fall }} \approx 50 M_{6}^{5 / 2} r_{\mathrm{p}}^{3} r_{*}^{-3 / 2} \mathrm{~s}
$$

where $M_{6}=M_{\mathrm{BH}} / 10^{6} M_{\odot}, r_{*}=R_{*} / R_{\odot}$ and $r_{\mathrm{p}}=R_{\mathrm{p}} / R_{\mathrm{S}}$ (Strubbe \& Quataert 2009), although $t_{\text {fall }}$ depends on the stellar density profile and can be evaluated more precisely 


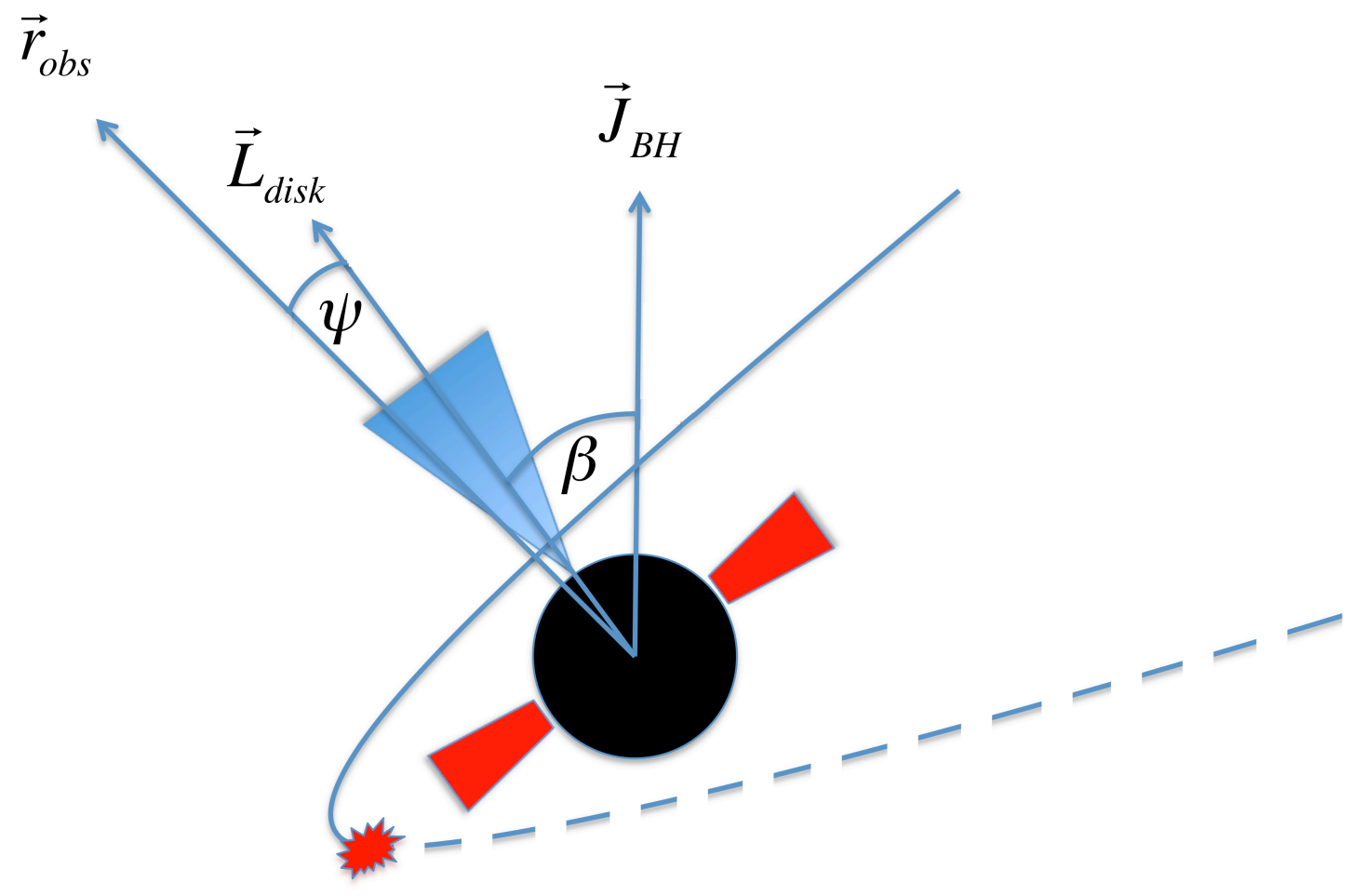

Figure 6.1.- : Geometry of the tidal disruption of a star by a spinning SMBH. Following disruption of the star near its pericenter passage, an accretion disk will form in the star's orbital plane. As the disk precesses, the angle $\beta$ between the SMBH spin vector $\vec{J}_{\mathrm{BH}}$ and the disk angular momentum vector $\vec{L}_{\text {disk }}$ stays constant, but an associated jet may move relative to the observer's line of sight $\vec{r}_{\text {obs }}$. 


\section{CHAPTER 6. DISK PRECESSION IN TDES}

by numerical simulations (Laguna et al. 1993). After a small multiple of this time, stream-stream collisions circularize the returning gas and allow an accretion disk to form. In general, this transient accretion disk will not lie in the black hole equatorial plane.

An accretion disk inclined out of the equatorial plane of a spinning black hole by an angle $\beta$ (assumed to equal the inclination of the stellar orbit before disruption, $\beta_{*}$ see Fig. 6.1) will be subject to Lense-Thirring torques with a strong radial dependence. For a thin disk (Kumar \& Pringle 1985), it is expected that the Bardeen-Petterson effect (Bardeen \& Petterson 1975; Papaloizou \& Pringle 1983) will induce a warp in the disk structure. However, for the thicker disks expected in many TDEs (Ulmer 1999; Strubbe \& Quataert 2009), simulations combining GR and magnetohydrodynamic effects (GRMHD) have shown that the disk precesses as a solid body rotator (Fragile et al. 2007; Dexter \& Fragile 2011). Such an accretion disk will precess with a period $T_{\text {prec }}=2 \pi \sin \beta(J / \tau)$, where $J$ is total angular momentum and $\tau$ is integrated torque. A notable feature of this formula is that $T_{\text {prec }}$ is independent of many disk model parameters, and depends only on the dimensionless radial surface density profile.

The simulations mentioned above considered disks with a roughly constant surface density. For surface densities of the form $\Sigma=\Sigma_{\mathrm{i}}\left(R / R_{\mathrm{i}}\right)^{-\zeta}$, the precession timescale is (Fragile et al. 2007)

$$
T_{\text {prec }}=\frac{8 \pi G M_{\mathrm{BH}}(1+2 \zeta)}{c^{3}(5-2 \zeta)} \frac{r_{\mathrm{o}}^{5 / 2-\zeta} r_{\mathrm{i}}^{1 / 2+\zeta}\left(1-\left(r_{\mathrm{i}} / r_{\mathrm{o}}\right)^{5 / 2-\zeta}\right)}{a\left(1-\left(r_{\mathrm{i}} / r_{\mathrm{o}}\right)^{1 / 2+\zeta}\right)} .
$$

Here the disk inner $\left(R_{\mathrm{i}}\right)$ and outer $\left(R_{\mathrm{o}}\right)$ edges have been normalized to units of Schwarzschild radii $\left(r_{\mathrm{i}}=R_{\mathrm{i}} / R_{\mathrm{S}}, r_{\mathrm{o}}=R_{\mathrm{o}} / R_{\mathrm{S}}\right)$. The variable $a$ is the dimensionless black hole spin parameter, with values between 0 and 1.

Whether or not the disks associated with tidal disruption flares approximately 


\section{CHAPTER 6. DISK PRECESSION IN TDES}

follow a surface density profile $\Sigma=\Sigma_{\mathrm{i}}\left(R / R_{\mathrm{i}}\right)^{-\zeta}$ is unclear. Strubbe \& Quataert (2009) presented a slim disk model for TDE accretion flows, with height $H$ given by:

$$
\frac{H}{R}=\frac{3 f}{4} \frac{10 \dot{M}}{\dot{M}_{\mathrm{Edd}}} \frac{R_{\mathrm{S}}}{R} K^{-1},
$$

where the function $K$ is defined as

$$
K=\frac{1}{2}+\sqrt{\frac{1}{4}+\frac{3 f}{2}\left(\frac{10 \dot{M}}{\dot{M}_{\mathrm{Edd}}}\right)^{2}\left(\frac{R_{\mathrm{S}}}{R}\right)^{2}} .
$$

Here $f=1-\left(R_{\mathrm{i}} / R\right)^{1 / 2} \cdot \dot{M} / \dot{M}_{\mathrm{Edd}}$ is the ratio of the mass accretion rate to the Eddington rate assuming $10 \%$ accretion efficiency.

However, this model is not suitable for use in calculating $T_{\text {prec }}$, as the zero-torque boundary condition used to calculate $f$ leads to an unphysical singularity in $\Sigma$ at $R_{\mathrm{i}}$ $\left(\Sigma \propto R^{3 / 2} K^{2} / f\right)$. A different, numerical model was recently presented in Montesinos Armijo \& de Freitas Pacheco (2011), in which axisymmetric disk equations were evolved with a time-dependent rate of mass input at the pericenter of disruption. This model led to a shallow decline of $\Sigma$ with decreasing $r$ after the arrival of the inner edge of the accretion flow at the innermost stable circular orbit. Motivated by Strubbe \& Quataert (2009) and Montesinos Armijo \& de Freitas Pacheco (2011), we consider $\zeta=-3 / 2,0,1$ in this paper. $T_{\text {prec }}$ increases by a factor $\approx 7$ when going from the $\zeta=1$ to the $\zeta=-3 / 2$ model.

The framework we followed is based on two underlying assumptions: (i) a coherent accretion flow exists; and (ii) the flow is not susceptible to Bardeen-Petterson warps $(H / R \gtrsim \alpha$, where $\alpha$ is the dimensionless disk viscosity parameter). Assumption (i) is only valid after a time $t_{\text {circ }} \approx n_{\text {orb }} t_{\text {fall }}$, where $n_{\text {orb }}$ is the number of orbits required to circularize the most tightly bound debris streams (Ulmer 1999). A value of $n_{\text {orb }} \sim 1-10$ is often assumed in the TDE literature, but this quantity is poorly constrained and could be 
higher for large $a$ and $\beta_{*}$, where Lense-Thirring precession can delay the stream-stream collisions necessary for disk formation (Kochanek 1994). At later times, assumption (ii) will break down, as $\dot{M} / \dot{M}_{\text {Edd }}$ declines and the disk becomes geometrically thinner.

Adopting Eq. (6.4) for convenience, $H / R$ will fall below $\alpha$ after a time

$$
\begin{array}{r}
t_{\text {thin }}=t_{\text {fall }}\left(\frac{5}{2} \frac{f}{X} \frac{M_{*} / t_{\text {fall }}}{\dot{M}_{\text {Edd }}} \frac{R_{\mathrm{S}}}{R}\right)^{3 / 5} \\
\approx 0.3 M_{6}^{2 / 5} r_{\mathrm{p}}^{6 / 5} m_{*}^{3 / 5} r_{*}^{-3 / 5}\left(\frac{f}{X_{-1}} \frac{R_{\mathrm{S}}}{R}\right)^{3 / 5} \mathrm{yr},
\end{array}
$$

where the function $X \sim \alpha$ and is $X=\alpha /\left(1-8 \alpha^{2} / 3 f\right)$. Also note that $X_{-1}=X / 0.1$. TDE disks will precess as solid body rotators during the time range $t_{\text {circ }}<t<t_{\text {thin }}$ as illustrated in Fig. 6.2, which shows that for $M_{\mathrm{BH}} \lesssim 10^{7} M_{\odot}$ (and any realistic $R_{\mathrm{p}}$ ), solid body precession will occur for $\lesssim 1 \mathrm{yr}$.

\subsection{Angular Momenta of Returning Debris Streams.}

The evolution of the debris streams prior to their first return to pericenter has been studied in detail by Ref. (Kochanek 1994). The orbits of these streams, if non-equatorial, lack a constant orbital plane due to Lense-Thirring torques. The accretion disk is therefore fed by a supply of new gas with time-dependent angular momentum, which in

turn evolves the direction of $\vec{L}_{\text {disk}}$. In contrast to direct precession of the accretion disk, we call this effect "differential stream precession," or DSP. Although we will compute numerical general relativistic solutions for the DSP, we can gain valuable intution from a simpler, lowest order estimate in the post-Newtonian limit.

The angle by which the angular momentum vector of a debris stream will precess during an orbit of period $T$ will be $\phi_{\mathrm{orb}}(T) \approx \Delta \Omega \sin (\beta)=2 \pi \sin (\beta)\left(T / t_{\mathrm{LT}}\right)$, where $\Delta \Omega$ is 


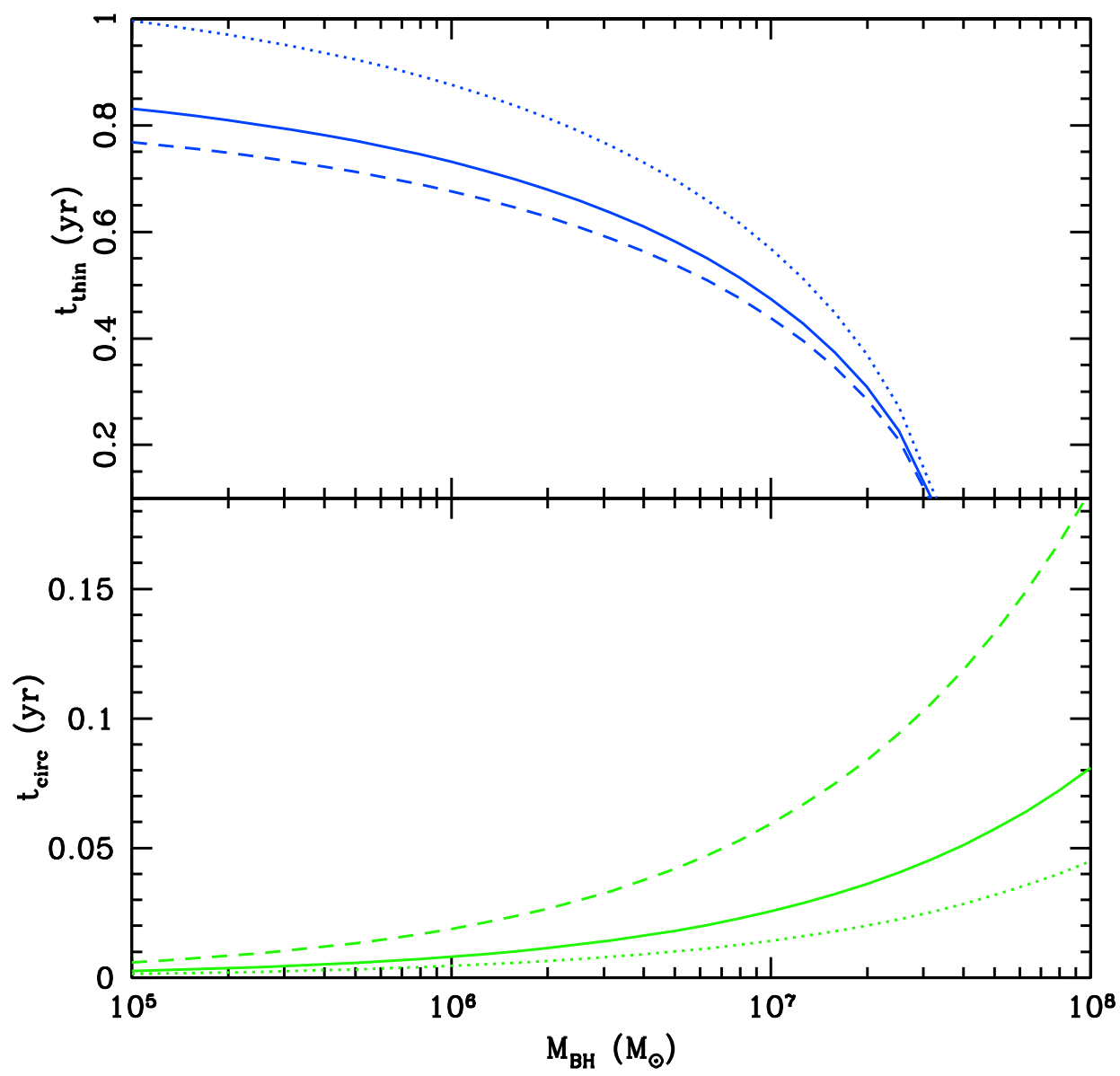

Figure 6.2.- : Timescales for avoiding Bardeen-Peterson warping $t_{\text {thin }}$ (blue, top panel) and for establishing an accretion disk $t_{\text {circ }}$ (green, bottom panel) as functions of the black hole mass $M_{\mathrm{BH}}$. Dotted lines correspond to stars with a mass of $2 M_{\odot}$, solid lines to $1 M_{\odot}$ and dashed lines to $0.5 M_{\odot}$, with a stellar mass-radius relationship adopted from Kippenhahn \& Weigert (1994), p. 208. We take $n_{\text {orb }}=3$ and $R_{\mathrm{p}}=0.5 R_{\mathrm{t}}$, and conservatively plot $t_{\text {thin }}$ for the outer edge of the disk, assuming $R_{\mathrm{o}}=2 R_{\mathrm{p}}$. 


\section{CHAPTER 6. DISK PRECESSION IN TDES}

the nodal precession and

$$
t_{\mathrm{LT}}=\frac{T}{2 a}\left(\frac{c^{2} A\left(1-e^{2}\right)}{G M_{\mathrm{BH}}}\right)^{3 / 2}
$$

is the Lense-Thirring precession period (Merritt et al. 2010) for a gas stream of semimajor axis $A$ and eccentricity $e$. Defining $\Delta \phi_{\text {orb }}=\phi_{\text {orb }}(T)-\phi_{\text {orb }}(\infty)$ as a measure of the DSP,

$$
\Delta \phi_{\text {orb }}=4 \pi a \sin (\beta)\left(2 r_{\mathrm{p}}\right)^{3 / 2}\left((1+e)^{-3 / 2}-2^{-3 / 2}\right)
$$

which Taylor expands in the late-time, $R_{\mathrm{p}} / A \ll 1$ limit to $\Delta \phi_{\text {orb }} \approx 1.7 \sin (\beta) a r_{\mathrm{p}}^{-5 / 2}$ $\times r_{*} M_{6}^{-1}\left(t / t_{\text {fall }}\right)^{-2 / 3}$.

Although Eq. (6.8) is not exact, it provides a valuable insight: the DSP is largest for low-mass, rapidly spinning SMBHs that disrupt stars with deeply plunging, inclined initial orbits. At early times the disk viscous timescale $t_{\text {visc }} \lesssim t_{\text {fall }}$ (Strubbe \& Quataert 2009) so Eq. (6.8) approximates the angular evolution of $\vec{L}_{\text {disk }}$. We do not expect $\Delta \phi_{\text {orb }}>1^{\circ}$ after the establishment of a steady accretion flow $\left(t>3 t_{\text {fall }}\right)$ for any TDEs with solar-type stars and $M_{6} \gtrsim 2$, although these constraints relax for stars with $r_{*}>1$.

To obtain an exact solution for the time evolution of angular momentum in the returning debris streams, a GR calculation is needed. We numerically integrate the Kerr geodesic equations following the formalism of Ref. (Drasco \& Hughes 2004). We assume a flat distribution of debris mass with specific Newtonian energy $E$, a spread in that energy of $3 G M_{\mathrm{BH}} R_{*} / R_{\mathrm{p}}^{2}$ (Strubbe \& Quataert 2009), and obtain constants of integration for each debris stream by transforming the initial conditions $\left\{E, R_{\mathrm{p}}, \beta\right\}$ to $\left\{E_{\mathrm{GR}}, L_{\mathrm{z}}, Q\right\}$ $\left(E_{\mathrm{GR}}, L_{\mathrm{z}}, Q\right.$ are specific energy, z-component angular momentum, and Carter's constant for Kerr metric test particles). Good agreement with Eq. (6.8) is shown in Fig. 6.3. 


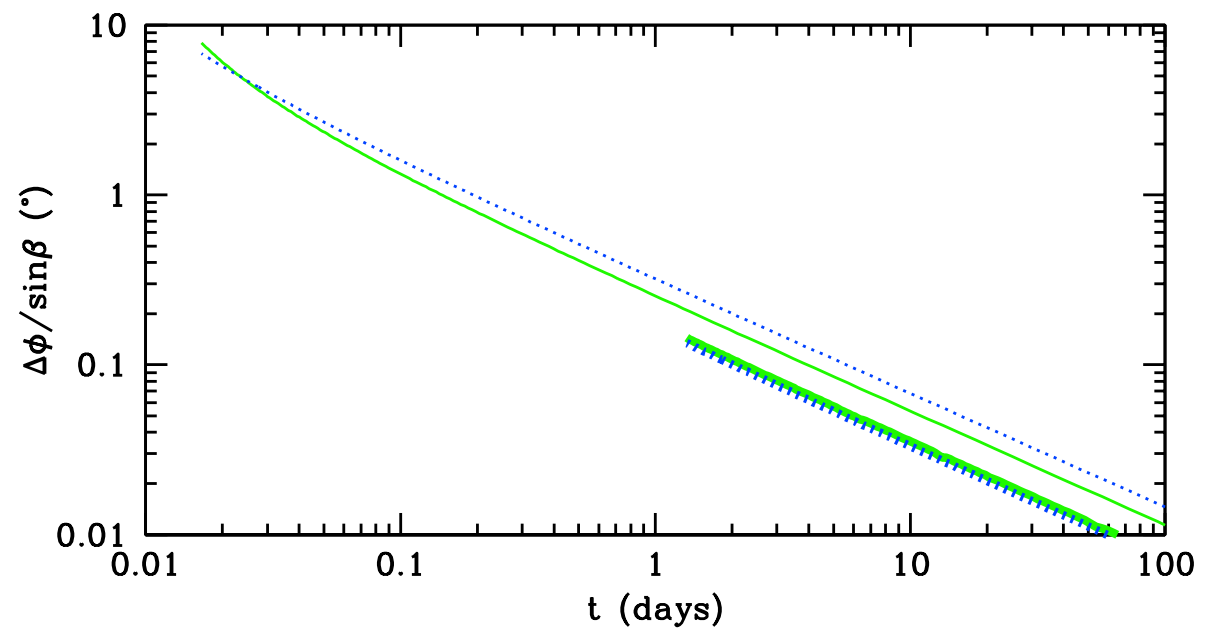

Figure 6.3.- : The angular shift $\Delta \phi_{\text {orb }}$. The thick curves illustrate the disruption of a solar-type star with $M_{\mathrm{BH}}=10^{6} M_{\odot}, a=0.8$, and $r_{\mathrm{p}}=13$; the thin curves are the same but with $r_{\mathrm{p}}=3$. The blue dotted lines are Eq. (6.8), while the green solid lines are numerical geodesic solutions. The curves do not extend prior to $t=t_{\text {fall }}$, and are normalized by $\sin \beta$. 


\section{CHAPTER 6. DISK PRECESSION IN TDES}

\subsection{Observational Implications.}

We have shown that the Lense-Thirring effect will cause the direction of a TDE disk's angular momentum vector to evolve in time. Direct precession of the accretion disk is the dominant effect, but in some cases DSP can cause a significant non-periodic evolution in $\vec{L}_{\text {disk }}$. The precession of the accretion disk will modulate the observed disk luminosity at least by a factor of $\cos (\psi)$, and lead to periodic pulsations of the associated transient quasar. This periodic modulation could in principle be extracted from the Fourier decomposition of a TDE lightcurve, but perhaps a more promising avenue for detection lies in the fraction of events for which the disks will precess into an edge-on phase. This could reduce the observed disk flux by $\sim 2$ orders of magnitude while simultaneously reddening the peak emission frequency (Ulmer 1999). Even in the absence of jet emission, observations of a "blinking" TDE flare could provide strong evidence of precession and allow both $a$ and the disruption parameters to be constrained.

The most exciting possible consequence, however, is precession of jets associated with TDE disks. If we assume that relativistic jets in tilted accretion systems align with $\vec{L}_{\text {disk }}$, narrow jets will precess out of the observer's line of sight in a small fraction of $T_{\text {prec }}$. Continuous observation of a jet for a relatively short period of time, $t_{\text {obs }}$, allows very strong constraints to be placed on combinations of $a$ and disruption parameters such as $r_{\mathrm{p}}$ and $\beta_{*}$ (assuming still that $\beta=\beta_{*}$ ). Alternatively, repeated observation of TDE-associated jets could serve as evidence that jets align with $\vec{J}_{\mathrm{BH}}$ or an aspect of the magnetic field geometry, provided that sufficient non-precession is observed. We note that the DSP, though generally subdominant, can in some cases cause very rapid precession (up to $\sim 0.1^{\circ} / \mathrm{min}$ ) at the onset of the flare (Fig. 6.3). If an associated jet is 


\section{CHAPTER 6. DISK PRECESSION IN TDES}

aligned with $\vec{L}_{\text {disk }}$, this will lead to a brief, nonrepeating transient which could fake an unusually long gamma ray burst provided $\theta_{\text {jet }} \lesssim 1^{\circ}$.

To provide a concrete example of the above considerations, we consider the tidal disruption candidate Swift J164449.3+573451, for which Zauderer et al. (2011) inferred the following relevant disruption parameters: $M_{\mathrm{BH}} \sim 10^{5}-10^{6} M_{\odot}, R_{\mathrm{p}} \approx 13 R_{\mathrm{S}} M_{6}^{-5 / 6}$, and $\theta_{\text {jet }} \sim 10^{-1.5}\left(\theta_{\text {jet }}\right.$ is estimated from both comparing the theoretical TDE rate to the observed rate of jets over the period of the Swift mission, and the Eddington limit of the $\mathrm{SMBH})$.

Figure 4 shows the resulting constraints on the joint $a-\beta$ parameter space of this TDE if we take $M_{6}=1$. Since the bright X-ray emission from Swift J164449.3+573451 persisted for over two weeks, at least one of the following statements must be true: (i) the value of $a$ is extremely low, $\lesssim 10^{-2}\left(10^{-1}\right.$ if $\left.\zeta=-3 / 2\right)$; (ii) the initial orbit of the disrupted star was tightly aligned to within $\sim \theta_{\text {jet }}$ of the black hole equatorial plane; or (iii) the jet emission was not aligned with the disk spin axis. The first possibility would represent an unusually low value of black hole spin and could be excluded if the Blandford-Znajek mechanism was responsible for jet launching (Lei \& Zhang 2011), while (ii) requires that there will be a larger abundance of somewhat shorter events. Since such flares are not frequently observed, the persistent X-ray emission in Swift J164449.3+573451 suggests that its jet was aligned with the steady spin axis of the black hole rather than with its precessing disk. Future GRMHD simulations can test this inference from first principles. The detection of additional TDE-associated jets in future surveys would test the statistical robustness of this conclusion. 


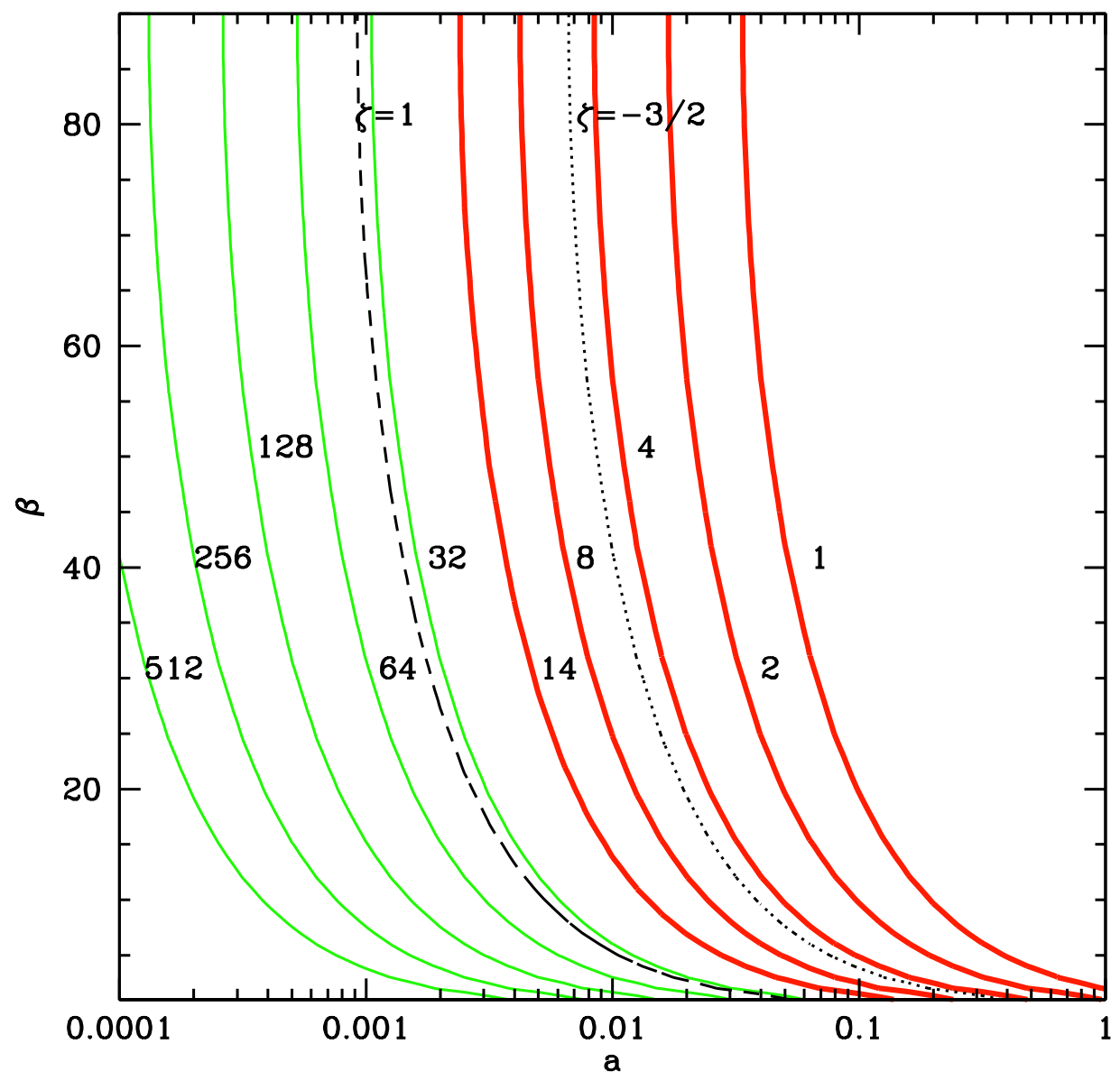

Figure 6.4.- : Regions of $a-\beta$ parameter space that can be excluded by continuous observations of a TDE jet with the inferred parameters in Zauderer et al. (2011) and $\zeta=0$. The solid curves show contours of constant $t_{\text {obs }}=T_{\text {prec }} \times 2\left(\theta_{\text {jet }} / 10^{-1.5}\right) /(2 \pi \sin \beta)$ : the maximum number of days it would take for a jet initially in the observers' line of sight to precess off-axis, with the jet opening angle normalized to $10^{-1.5}$. We take $R_{\mathrm{o}}=2 R_{\mathrm{p}}$ and $R_{\mathrm{i}}=3 R_{\mathrm{S}}$. Regions of parameter space to the right of the thick red contours can be excluded for the Swift TDE jet, which exhibited bright X-ray emission for over two weeks. The 14 day contours for $\zeta=-3 / 2$ and $\zeta=1$ are shown with black dotted and dashed lines, respectively. The effect of the DSP is negligible for these parameters, and neglected here. 


\title{
Chapter 7
}

\section{Pulsations in Short GRBs from}

\section{Black Hole-Neutron Star Mergers}

N. Stone, A. Loeb, \& E. Berger Physical Review D, Vol. 87, id. 084053, 2013

\begin{abstract}
The precise origin of short gamma ray bursts (SGRBs) remains an important open question in relativistic astrophysics. Increasingly, observational evidence suggests the merger of a binary compact object system as the source for most SGRBs, but it is currently unclear how to distinguish observationally between a binary neutron star progenitor and a black hole-neutron star progenitor. We suggest the quasi-periodic signal of jet precession as an observational signature of SGRBs originating in mixed binary systems, and quantify both the fraction of mixed binaries capable of producing SGRBs, and the distributions of precession amplitudes and periods. The difficulty inherent in
\end{abstract}




\section{CHAPTER \%. DISK PRECESSION IN SGRBS}

disrupting a neutron star outside the horizon of a stellar-mass black hole biases the jet precession signal towards low amplitude and high frequency. Precession periods of $\sim 0.01-0.1 \mathrm{~s}$ and disk-BH spin misalignments $\sim 10^{\circ}$ are generally expected, although sufficiently high viscosity may prevent the accumulation of multiple precession periods during the SGRB. The precessing jet will naturally cover a larger solid angle in the sky than would standard SGRB jets, enhancing observability for both prompt emission and optical afterglows.

\subsection{Introduction}

The origin of the short-hard gamma-ray bursts (SGRBs; durations $T_{90} \lesssim 2 \mathrm{~s}$; (Kouveliotou et al. 1993)) was largely a matter of speculation until the recent discovery of their afterglows and host galaxies (e.g., (Berger et al. 2005; Fox et al. 2005; Gehrels et al. 2005; Bloom et al. 2006)). These observations have demonstrated that SGRBs are cosmological in origin $(z \gtrsim 0.1$; (Berger et al. 2007)); have a beaming-corrected energy scale of $\sim 10^{49}-10^{50}$ erg (Burrows et al. 2006; Soderberg et al. 2006; Fong et al. 2012); lack associated supernovae (e.g., (Hjorth et al. 2005; Soderberg et al. 2006)); occur in a mix of star-forming and elliptical galaxies (Berger 2009); have a broad spatial distribution around their hosts (Fong et al. 2010), with some events offset by tens of kpc (Berger 2010); and have low-density parsec-scale environments (Soderberg et al. 2006; Fong et al. 2010). The confluence of these characteristics provides support to the popular model of compact object (CO) mergers (Paczynski 1991).

In this context, the key open question which motivates our paper is: if SGRBs originate in $\mathrm{CO}$ mergers, what types of compact objects are merging? Specifically, a 


\section{CHAPTER \%. DISK PRECESSION IN SGRBS}

neutron star (NS) can theoretically be tidally disrupted by, and produce an accretion disk around, either a more compact NS, or a sufficiently small stellar mass black hole (BH). Unfortunately, it is not clear how to distinguish between mergers of neutron star binaries (NS-NS) and mixed binaries (BH-NS). The advent of gravitational wave (GW) astronomy will facilitate this task, since the waveforms accompanying NS-NS mergers may be sufficiently distinct from those in BH-NS mergers, but the Advanced LIGO era is still half a decade away. It is also possible that GW signals seen by Advanced LIGO will lack accompanying electromagnetic counterparts if the SGRB beaming angle is too small, or the intrinsic event rate too low. In this paper we suggest a clear observational tool for distinguishing between NS-NS and BH-NS progenitors of SGRBs using electromagnetic data only: the precession of the disks and jets associated with these events.

Jet precession has previously been discussed as a phenomenon relevant for GRBs, originally with regard to now-disfavored GRB models (Roland et al. 1994; Blackman et al. 1996) but later in the context of CO mergers. Early works in the CO merger paradigm considered disks fed by stable mass transfer from a NS onto a $\mathrm{BH}$ so that the precession was forced by tidal torques (Portegies Zwart et al. 1999), but subsequent models considered the more realistic neutrino-dominated accretion flows (NDAFs) formed after tidal disruption of a NS by a stellar mass BH (Reynoso et al. 2006). This last model is similar to the one presented in this paper, in that it considers a thick disk precessing as a solid body rotator due to general relativistic Lense-Thirring torques. It has been applied to predict different observational signatures, such as the light curves of precessing jets (Reynoso et al. 2006; Lei et al. 2007) or even LIGO-band gravitational wave (GW) signals emitted by forced precession of a large amount of disk mass (Romero et al. 2010; Sun et al. 2012). Others have considered an inclined disk 


\section{CHAPTER \%. DISK PRECESSION IN SGRBS}

whose inner regions include a Bardeen-Petterson warp; in their model the inner region of a neutrino-dominated accretion flow (NDAF) precesses along with the $\mathrm{BH}$ spin about the total angular momentum vector (Liu et al. 2010).

This paper follows Reynoso et al. (2006) in focusing on thick disks precessing as solid body rotators, which are well-motivated for the supercritical accretion flows and misaligned angular momentum vectors characteristic of compact object mergers (§2). Our work differs from past efforts, however, in our consideration of the viscous spreading of the disk, as well as our adoption of simplifying assumptions tailored to match results from numerical relativity (NR) simulations of mixed binary mergers (§3). We quantify for the first time the distributions of precession periods and angles given physically motivated assumptions about progenitor spins and masses $(\S 4)$, using simple analytic formulae when appropriate and more complex empirical fits to NR simulations when necessary. In the process we estimate the fraction of BH-NS mergers which can actually produce accretion disks. We conclude by considering the observable consequences of jet precession in the context of SGRBs $(\S 5)$. Unlike in previous work, we discuss both the case where the jet is tied to the $\mathrm{BH}$ spin vector, and the case where it aligns with the disk angular momentum vector.

\subsection{Disk Precession}

Non-axisymmetric torques will, initially, induce small warps in accretion disks due to differential precession between adjacent mass annuli. The evolution of these warps depends on how they are able to propagate through the disk. When the timescale for precession is shorter than the sound crossing time, these warps will propagate in 


\section{CHAPTER \%. DISK PRECESSION IN SGRBS}

a diffusive way, allowing differential precession to produce substantial shear viscosity and dissipating large amounts of orbital energy. In the context of tilted accretion disks around spinning black holes experiencing Lense-Thirring torques, diffusive propagation of warps will align the inner regions of the disk with the black hole equatorial plane; this is known as the Bardeen-Petterson effect (Bardeen \& Petterson 1975; Papaloizou \& Pringle 1983; Ogilvie 1999).

In the opposite regime, a thick disk with a short sound-crossing timescale will propagate warps in a wavelike manner, redistributing torques throughout the disk and inducing near-rigid body rotation (Papaloizou \& Lin 1995; Papaloizou \& Terquem 1995). In particular, rigid body rotation is possible if $H / r>\alpha$, where $H / r$ is the disk height and $\alpha$ the dimensionless viscosity parameter at a radius $r$. This is the regime most relevant for compact object mergers, and is therefore what we will consider for the remainder of this paper. Approximately rigid body precession has been seen in hydrodynamical simulations of protoplanetary disks being torqued by a binary companion (Larwood et al. 1996), in GRMHD simulations of tilted accretion disks around spinning black holes (Fragile et al. 2007; Fragile \& Blaes 2008), and, notably, in NR simulations of BH-NS mergers (Foucart et al. 2011).

In the Newtonian limit, a solid body rotator will precess with a period $T_{\text {prec }}=2 \pi \sin \psi_{\mathrm{d}}(J / \tau)$, where $\psi_{\mathrm{d}}$ is the misalignment angle between the accretion disk and the $\mathrm{BH}$ equatorial plane, $J$ is the total angular momentum of the disk, and $\tau$ is the Lense-Thirring torque integrated over the entire disk. Specifically, if the disk possesses a surface density profile $\Sigma(r)$ that is nonzero from inner radius $R_{\mathrm{i}}$ to outer radius $R_{\mathrm{o}}$, and 


\section{CHAPTER \%. DISK PRECESSION IN SGRBS}

the disk elements possess orbital frequency $\Omega(r)$, then

$$
J=2 \pi \int_{R_{\mathrm{i}}}^{R_{\circ}} \Sigma(r) \Omega(r) r^{3} \mathrm{~d} r
$$

and

$$
\tau=4 \pi \frac{G^{2} M_{\mathrm{BH}}^{2} a_{\mathrm{BH}}}{c^{3}} \sin \psi_{\mathrm{d}} \int_{R_{\mathrm{i}}}^{R_{\mathrm{o}}} \frac{1}{r^{3}} \Sigma(r) \Omega(r) r^{3} \mathrm{~d} r,
$$

where the BH's mass and dimensionless spin are $M_{\mathrm{BH}}$ and $a_{\mathrm{BH}}$, respectively. Throughout this paper $G$ is the gravitational constant and $c$ is the speed of light. If we use the Keplerian orbital frequency $\Omega_{\mathrm{k}}=\sqrt{G M_{\mathrm{BH}} / r^{3}}$, then for a density profile of the form $\Sigma(r)=\Sigma_{0}\left(r / r_{0}\right)^{-\zeta}$, the precession timescale is

$$
T_{\text {prec }}=\frac{\pi r_{\mathrm{g}}(1+2 \zeta)}{c(5-2 \zeta)} \frac{r_{\mathrm{o}}^{5 / 2-\zeta} r_{\mathrm{i}}^{1 / 2+\zeta}\left(1-\left(r_{\mathrm{i}} / r_{\mathrm{o}}\right)^{5 / 2-\zeta}\right)}{a_{\mathrm{BH}}\left(1-\left(r_{\mathrm{i}} / r_{\mathrm{o}}\right)^{1 / 2+\zeta}\right)} .
$$

Here we have normalized $r_{\mathrm{o}}=R_{\mathrm{o}} / r_{\mathrm{g}}$ and $r_{\mathrm{i}}=R_{\mathrm{i}} / r_{\mathrm{g}}$ by the gravitational radius $r_{\mathrm{g}}=G M_{\mathrm{BH}} / c^{2}$. Other effects which influence Eq. (7.3) include nutation and relativistic corrections to the orbital frequency, which we neglect in this analysis - but see Portegies Zwart et al. (1999); Reynoso et al. (2006).

We stress that $T_{\text {prec }}$ is an instantaneous precession period, and that for the non-steady state disks expected in CO mergers important quantities such as $\zeta$ and $r_{\mathrm{o}}$ will be time-dependent (although the cancellation of $\Sigma_{0}$ in $J / \tau$ means that the secular decrease in disk mass will not affect $\left.T_{\text {prec}}\right)$. The time dependence of these variables will cause any signal to be quasi-periodic rather than periodic. Because the dominant feature of the disk's evolution will be viscous outward spreading (Metzger et al. 2008, 2009), we expect $T_{\text {prec }}$ to increase with time.

Although a power law definition of $\Sigma$ is appealing for its simplicity, both analytical models (Metzger et al. 2009) and NR simulations (Foucart et al. 2011) indicate that 


\section{CHAPTER \%. DISK PRECESSION IN SGRBS}

the true structure of these disks is more complex. To better account for realistic disk structure, and also to quantify the time evolution of the disk as it spreads outward, we adopt the SGRB disk model of Metzger et al. (2008), which derives exact $\Sigma$ solutions for a viscously spreading ring of matter, and then couples these solutions to more detailed models of disk energetics and composition. As matter from the disk accretes onto the $\mathrm{BH}$, the intially advective, neutrino-dominated accretion flow will become optically thin to neutrinos and geometrically thinner. At later times $(t \gtrsim 0.1 \mathrm{sec})$ the disk will become a geometrically thick, radiatively-inefficient accretion flow (RIAF). In principle, the intermediate neutrino-cooled period could prevent later disk precession by aligning the disk into the $\mathrm{BH}$ midplane through the creation of a Bardeen-Petterson warp. In practice, it seems that even the neutrino-cooled phase of accretion still possesses $H / r>\alpha$, and is therefore unlikely to align - see Metzger et al. (2009), Fig. 2.

We are primarily interested in the RIAF stage, both because it has the longest duration, and because for low disk masses $\left(M_{\mathrm{d}} \lesssim 0.1 M_{\mathrm{NS}}\right.$, which is the case for most BH-NS mergers - see $\S I I I)$, it is the only phase of accretion. For this stage of disk evolution, we can write the surface density as

$$
\begin{aligned}
\Sigma(r, t)= & \frac{M_{\mathrm{d}}(1-n / 2)}{\pi R_{\mathrm{dis}}^{2} x^{n+1 / 4} \tau} \exp \left(\frac{-\left(1+x^{2-n}\right)}{\tau}\right) \\
& \times I_{1 /|4-2 n|}\left(\frac{2 x^{1-n / 2}}{\tau}\right) .
\end{aligned}
$$

Here $M_{\mathrm{d}}$ is the initial disk mass, $R_{\mathrm{dis}}$ is the initial radius of the spreading mass ring (i.e. the radius where the NS is disrupted), $I_{m}$ is a modified Bessel function of order

$m, x=r / R_{\mathrm{dis}}, \tau=t\left(12 \nu_{0}(1-n / 2)^{2} / R_{\mathrm{dis}}^{2}\right)$, and we have assumed viscosity of the form 


\section{CHAPTER \%. DISK PRECESSION IN SGRBS}

$\nu=\nu_{0} x^{n}$. We calibrate $\nu_{0}$ with the initial relation $t_{\mathrm{visc}, 0}=R_{\mathrm{dis}}^{2} / \nu$ and the equation

$$
\begin{aligned}
t_{\mathrm{visc}, 0} \approx 6 \times 10^{-2}\left(\frac{\alpha}{0.1}\right)^{-1} & \left(\frac{M_{\mathrm{BH}}}{3 M_{\odot}}\right)^{-1 / 2}\left(\frac{R_{\mathrm{dis}}}{10^{5} \mathrm{~m}}\right)^{3 / 2} \\
& \times\left(\frac{H_{0}}{0.3 R_{\mathrm{dis}}}\right)^{-2} \mathrm{~s},
\end{aligned}
$$

where $\alpha$ is the dimensionless Shakura-Sunyaev viscosity coefficient and $H_{0}$ is the characteristic disk height. The value of $\alpha$ is set by the magneto-rotational instability $(\mathrm{MRI})$, and has been estimated to span a wide range of values, from $\sim 0.01$ in local, shearing box simulations (Davis et al. 2010) to $\sim 1$ in global GRMHD simulations (McKinney \& Narayan 2007). However, large $\alpha$ values seen in global simulations are confined to small radii, and in these simulations $\alpha \sim 0.1$ at $r \gtrsim 10 r_{\mathrm{g}}$. The importance of $\alpha$ for our results lies primarily in how viscosity controls the outward spreading of the disk, so we follow Ref. (Chen \& Beloborodov 2007) and consider large-radii $\alpha$ values of $0.01,0.03$, and $0.1 . H_{0}$ will vary both in radius and in time; generally, $H_{0}$ grows as one moves further out in the disk (Chen \& Beloborodov 2007), and also as the outer edge of the disk viscously spreads, putting a larger fraction of the disk into a purely advective regime with large height (Metzger et al. 2008). Our results are fairly sensitive to both $\alpha$ and $H_{0}$, but because the former spans a wider range we vary $\alpha$ and fix $H_{0}=0.3 R$. We arrive at this value by considering the size of the disk at a time $t_{1 / 2}$, a characteristic, "halfway," precession timescale. Specifically, $t_{1 / 2}=\left(\left(t_{\mathrm{visc}, 0}^{-1 / 3}+1\right) / 2\right)^{-3}$ is the time at which half the SGRB's precession cycles will have occurred if it lasts from $t=t_{\mathrm{visc}, 0}$ to $t=1 \mathrm{~s}$ and viscous spreading of the disk causes $T_{\text {prec }} \propto t^{4 / 3}$. Typically, $t_{1 / 2} \sim 100$ msec, which corresponds to a disk outer edge at $r_{\mathrm{o}} \sim 50$; at this distance, more detailed modeling of disk structure (Chen \& Beloborodov 2007) indicates $H / R \approx 0.3$.

Using Eq. (7.4), we plot the time evolution of $T_{\text {prec }}$ in Figure 7.1, and find that 


\section{CHAPTER \%. DISK PRECESSION IN SGRBS}

it increases in rough agreement with analytic expectations: at late times, Eq. (7.4) approaches a power law with $\zeta=1 / 2$, and the outer edge of the disk $r_{\mathrm{o}} \propto t^{2 / 3}$. Assuming that $r_{\mathrm{i}}$ remains fixed (and ignoring lower-order contributions from $r_{\mathrm{o}}$ ), Eq. (7.3) then implies $T_{\text {prec }} \propto t^{4 / 3}$. In Fig. 7.1 we also plot $N_{\text {cycles }}$, the total number of precession cycles undergone during the GRB. For $\alpha \gtrsim 0.1$, a viscously spreading SGRB disk will generally experience $N_{\text {cycles }} \lesssim 1$.

In the above discussion we have assumed that the angular momentum lost by inspiralling disk matter is redistributed outward by internal viscous torques. An alternate possibility is angular momentum loss through a magnetized disk wind (Metzger et al. 2008), in which case $r_{\mathrm{o}} \propto t^{2 / 5}$ and $T_{\text {prec }} \propto t^{4 / 5}$. If this is the case, $N_{\text {cycles }} \gtrsim 10$ for all realistic $\alpha$ values. For the remainder of this paper, however, we conservatively calculate fiducial precession timescales using Eq. (7.4) and $n=1 / 2$. Our results are generally insensitive to $R_{\mathrm{dis}}$ and independent of $M_{\mathrm{d}}$ (although we will later use $M_{\mathrm{d}}$ as a criterion for whether or not an SGRB can form - see §III). On the other hand, our results are fairly sensitive to $\alpha$, with large $\alpha$ values increasing the precession period and decreasing the number of precession cycles that can fit in the duration of an SGRB. In all cases we self-consistently calculate the inner disk edge $r_{\mathrm{i}}$ using the formalism in Perez-Giz $2013^{1}$ for finding the innermost stable spherical orbit (ISSO), the tilted analogue to the innermost stable circular orbit (ISCO). Details of the ISSO calculation are in Appendix C.

The precession of the SGRB disk in isolation is unlikely to be observable, and is mainly interesting as a source of jet precession. The observational signatures of jet

\footnotetext{
${ }^{1}$ Work in preparation.
} 


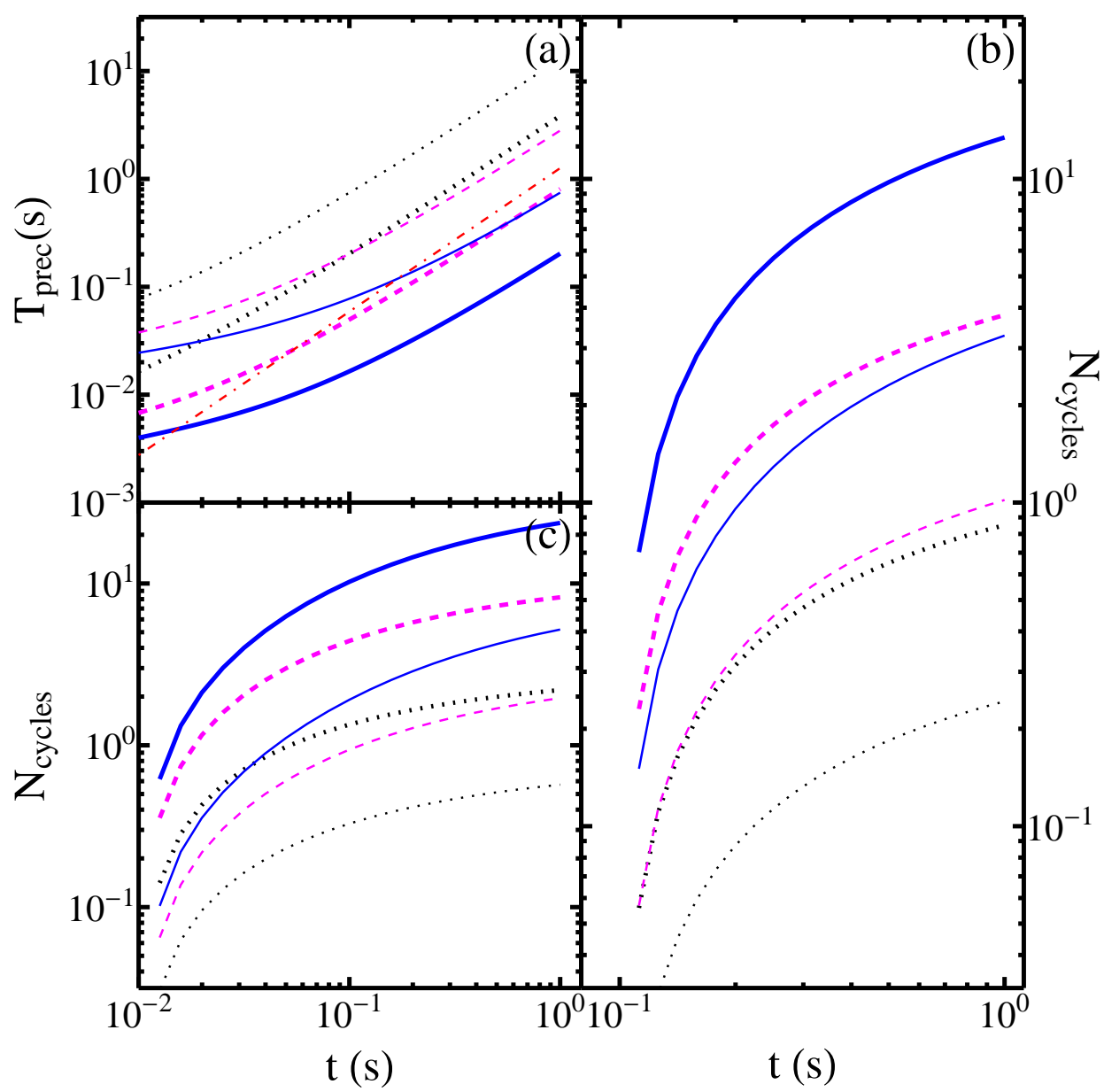

Figure 7.1.- : Panel (a) shows the time evolution of $T_{\text {prec }}$ assuming a viscously spreading disk structure given by Eq. (7.4). The black dotted curves represent $\alpha=0.1$, the dashed magenta curves $\alpha=0.03$, and the solid cyan curves $\alpha=0.01$. Thick curves are for nearly equatorial disruptions with $a_{\mathrm{BH}}=0.9$, while thin curves are for $a_{\mathrm{BH}}=0.9$ and initial spin-orbit misalignment of $70^{\circ}$, or equivalently a nearly aligned disruption with $a \approx 0.5$. The dash-dotted red line is $\propto t^{4 / 3}$, the rough time evolution of $T_{\text {prec }}$. Panels (b) and (c) show $N_{\text {cycles }}$, the accumulated number of cycles for $0.1 \mathrm{~s}<t<1 \mathrm{~s}$ and $0.01 \mathrm{~s}<t<1 \mathrm{~s}$, respectively. 


\section{CHAPTER \%. DISK PRECESSION IN SGRBS}

precession will hinge on two uncertain astrophysical questions: the opening angles of SGRB jets, and the alignment direction of a jet in a tilted accretion flow. The first of these questions has recently become amenable to observational constraint; observations of jet breaks in SGRBs suggest opening angles of $\sim 10^{\circ}$ (Soderberg et al. 2006). Observational evidence for the second question is limited, and ambiguous. Observations of a relativistic outflow following tidal disruption of a star by a supermassive BH (Swift J164449.3+573451) suggested that in that case, the jet aligned with the BH spin axis (Stone \& Loeb 2012a); on the other hand, observations of the microquasar LSI+61303 have been interpreted as evidence of a precessing jet, aligned with the angular momentum axis of a precessing disk (Massi et al. 2012). There may not be a universal answer to this question, as different hypothetical jet launching mechanisms might each tie the jet axis to a different preferred direction.

However, for the two leading jet launching mechanism candidates in SGRBs $-\nu \bar{\nu}$ pair annihilation (Meszaros \& Rees 1992; Ruffert \& Janka 1999), and the Blandford-Znajek (BZ) mechanism (Blandford \& Znajek 1977; Lee et al. 2000) - there are theoretical reasons to believe that the jet will align with the disk angular momentum vector. The $\nu \bar{\nu}$ annihilation scenario is independent of $\mathrm{BH}$ spin and depends only on disk properties. Alignment of a BZ-powered jet is more ambiguous, but recent works that have considered jet precession in SGRBs assumed that a jet powered by the BZ mechanism will align with the disk angular momentum vector (Reynoso et al. 2006; Lei et al. 2007) because the magnetic field is anchored in the disk. This assumption has been further supported by NR simulations of force-free electromagnetic fields around spinning BHs (Palenzuela et al. 2010), which found that the direction of Poynting flux from the BZ mechanism is governed by larger-scale magnetic fields and not the BH spin vector, although we note 
that GRMHD simulations of tilted accretion flows with matter have so far been unable to resolve jets (Fragile 2008).

\subsection{Progenitor Binaries}

Because large amplitude precession requires large amplitude misalignment of the post-merger $\mathrm{BH}$ and its accretion disk, we must consider which $\mathrm{CO}$ mergers can actually produce misaligned disks. Despite their larger pre-merger spin-orbit misalignment, NS-NS mergers are unlikely to produce significantly misaligned disks. If one or both members of the NS-NS binary were millisecond pulsars, disk precession could be feasible: spin angular

momentum $J_{\mathrm{MSP}} \approx \frac{2}{5} M_{\mathrm{NS}} R_{\mathrm{NS}}^{2} \Omega_{\mathrm{NS}} \approx 1 \times 10^{42} \mathrm{~kg} \mathrm{~m} \mathrm{~m}^{2} / \mathrm{s}$, and the orbital angular momentum at the disruption radius $L_{\mathrm{NS}-\mathrm{NS}} \approx 2 M_{\mathrm{NS}}\left(R_{\mathrm{NS}}\right)^{2} \sqrt{2 G M_{\mathrm{NS}} /\left(R_{\mathrm{NS}}\right)^{3}} \approx 1 \times 10^{43} \mathrm{~kg} \mathrm{~m}^{2} / \mathrm{s}$. Thus, the misalignment angle $\psi_{\mathrm{d}}$ between the post-merger accretion disks (which we assume to lie in the initial orbital plane) and the $\mathrm{BH}$ spin axis will be $\sim 5^{\circ}$ in NS-NS mergers involving one star with a ms spin period, if we assume initially orthogonal spin and orbital angular momentum vectors. Standard population synthesis channels indicate, however, that the one recycled component of NS binaries typically has a minimum spin period of $\approx 4 \mathrm{~ms}$ (Willems et al. 2008), which would imply $\psi_{\mathrm{d}} \lesssim 1^{\circ}$, a value that is likely too small to carry significant observational consequences.

In BH-NS mergers, however, the BH may possess a larger natal reservoir of spin angular momentum, allowing for greater misalignment between the post-merger $\mathrm{BH}$ and the disk formed from NS debris (which we have assumed to lie in the initial orbital plane). Natal spin is the most relevant quantity, although subsequent mass transfer onto the $\mathrm{BH}$ may produce modest changes in $a_{\mathrm{BH}}$ (Belczynski et al. 


\section{CHAPTER \%. DISK PRECESSION IN SGRBS}

2008). For a BH-NS system, the relevant numbers are $J_{\mathrm{BH}}=a_{\mathrm{BH}} G M_{\mathrm{BH}} / c$ and $L_{\mathrm{BH}-\mathrm{NS}} \sim(1+q) M_{\mathrm{BH}}\left(R_{\mathrm{NS}}\right)^{2} \sqrt{(1+q) G M_{\mathrm{BH}} /\left(R_{\mathrm{NS}}\right)^{3}}$, where $a_{\mathrm{BH}}$ is the dimensionless black hole spin and we define the mass ratio $q=M_{\mathrm{NS}} / M_{\mathrm{BH}}$. For $M_{\mathrm{BH}}=5 M_{\odot}\left(10 M_{\odot}\right)$ and $a_{\mathrm{BH}}=0.9$, the disk misalignment angle $\psi_{\mathrm{d}} \lesssim 20^{\circ}\left(30^{\circ}\right)$, which is large enough to be observationally interesting. Here we have also assumed initially orthogonal spin and orbital angular momentum vectors.

These simple Newtonian estimates motivate an investigation of BH-disk misalignment in BH-NS mergers, but are insufficient for accurately estimating either post-merger BH spin $\vec{a}_{\mathrm{BH}}^{\prime}$ or the misalignment angle $\psi_{\mathrm{d}}$ between $\vec{a}_{\mathrm{BH}}^{\prime}$ and $\vec{L}_{\mathrm{BH}-\mathrm{NS}}$. This is because they do not account for the fully dynamical, strong field GR effects that accompany compact object mergers, and as we shall see underestimate post-merger misalignment. Recently, empirical post-Newtonian (PN) formulas were derived to calculate these quantities in the case of BH-BH mergers (Lousto et al. 2010a). These formulas have a large number of free parameters which were calibrated based on a suite of numerical relativity (NR) simulations. Because disruptions of NSs by BHs are so marginal (i.e. occur so close to the ISSO), it is reasonable to expect these formulas to have some utility in making predictions for BH-NS mergers; we show in Appendix D that they are in fact quite accurate when compared to NR simulations of BH-NS coalescence. For this reason, we use these PN formulae to calculate the final spin $\vec{a}_{\mathrm{BH}}^{\prime}$ for a $\mathrm{BH}$ formed by the merger of a BH-NS with mass ratio $q$, pre-merger $\mathrm{BH}$ spin $\vec{a}_{\mathrm{BH}}$, and a pre-merger spin-orbit misalignment angle $\psi$. We approximate the pre-merger NS spin magnitude as $\vec{a}_{\mathrm{NS}}=0$. Finally, we calculate $\cos \psi_{\mathrm{d}}=\vec{a}_{\mathrm{BH}}^{\prime} \cdot \vec{L}_{\mathrm{BH}-\mathrm{NS}} /\left(a_{\mathrm{BH}}^{\prime} L_{\mathrm{BH}-\mathrm{NS}}\right)$.

Even though the PN fomulae in Appendix D reproduce $\vec{a}_{\mathrm{BH}}^{\prime}$ with reasonable accuracy, our assumption that the post-merger disk tilt $\psi_{\mathrm{d}}$ is described by the angle 


\section{CHAPTER \%. DISK PRECESSION IN SGRBS}

between the initial orbital plane and $\vec{a}_{\mathrm{BH}}^{\prime}$ may overestimate $\psi_{\mathrm{d}}$. When we compare our calculations to the late-time (40 ms) results of Foucart et al. (2011), we appear to overestimate $\psi_{\mathrm{d}}$ by a factor $\sim 3$, although our approach provides a significantly more accurate estimate of early-time disk tilt. Similar evolution of disk tilt is not seen in steady-state GRMHD disk simulations (Fragile et al. 2007), but is likely at least partially physical for BH-NS mergers, as the disk adjusts to an equilibrium configuration. However, numerical viscosity may also play a role in reducing the disk tilt in misaligned NR simulations ${ }^{2}$. For simplicity, we do not model the time evolution of $\psi_{\mathrm{d}}$, but this probably results in overestimates of $\approx 2$, which we note again in $\S \mathrm{V}$.

The final component in our calculation is a criterion for SGRB production in a BH-NS merger. The tidal radius, defined in the Newtonian limit as $r_{\mathrm{t}}=R_{\mathrm{NS}} q^{-1 / 3}$, is appealing for this purpose: only a fraction $f_{\mathrm{GRB}}$ of all BH-NS mergers will produce an accretion disk and jet, because if $r_{\mathrm{t}}<r_{\mathrm{ISSO}}$, the NS is swallowed whole. However, while the tidal radius is cleanly defined in other contexts (Rees 1988), in the case of relativistic, comparable mass mergers it is not obvious that the Newtonian definition is applicable (Fishbone 1973). Furthermore, the gravitational radius $r_{\mathrm{g}} \sim R_{\mathrm{NS}}$. In light of these complications, we adopt a fitting formula for the initial remnant disk mass $M_{\mathrm{d}}$, calibrated from NR simulations of aligned BH-NS mergers (Foucart 2012):

$$
\frac{M_{\mathrm{d}}}{M_{\mathrm{NS}}}=0.415 q^{-1 / 3}\left(1-2 \frac{G M_{\mathrm{NS}}}{c^{2} R_{\mathrm{NS}}}\right)-0.148 \frac{r_{\mathrm{ISCO}}}{R_{\mathrm{NS}}} .
$$

Although this fitting formula was calibrated from NR data on aligned mergers, we generalize it to misaligned mergers by substituting $r_{\text {ISSO }}$ for $r_{\text {ISCO }}$. This appears to reproduce NR simulations of misaligned postmerger disks reasonably well (Appendix D).

\footnotetext{
${ }^{2}$ Francois Foucart, personal communication.
} 


\section{CHAPTER \%. DISK PRECESSION IN SGRBS}

The limiting value of $M_{\mathrm{d}} / M_{\mathrm{NS}}$ required to produce a SGRB is highly uncertain, but past theoretical work assuming jets are powered by $\nu \bar{\nu}$ annihilation has suggested that SGRBs are viable for $M_{\mathrm{d}} / M_{\mathrm{NS}}>0.01$ (Ruffert \& Janka 1999); likewise, a recent attempt to observationally infer disk masses assuming $\nu \bar{\nu}$ annhilation (Fan \& Wei 2011) found $0.01<M_{\mathrm{d}} / M_{\mathrm{NS}}<0.1$. In this work, we take $M_{\mathrm{d}} / M_{\mathrm{NS}}>0.05$ as the cutoff for SGRB production, but discuss the effects of stricter and weaker criteria in $\S \mathrm{IV}$ and V.

\subsection{Distributions}

We now integrate the above analytic criteria over distributions of progenitor masses and spins to find distributions of $f_{\mathrm{GRB}}, \psi_{\mathrm{d}}, N_{\text {cycles }}$, and $T_{\text {prec }}\left(t_{1 / 2}\right)$. Because the distributions of progenitor quantities are not at present precisely constrained by observation or population synthesis, we consider a wide range of possibilities to bracket the available parameter space. For our fiducial case, we take the parametric BH mass function from Özel et al. (2010) (hereafter the "OPNM mass function"), given by

$$
P_{\mathrm{OPNM}}\left(M_{\mathrm{BH}}\right)=\frac{e^{M_{\mathrm{c}} / M_{\text {scale }}}}{M_{\text {scale }}} \begin{cases}e^{-M / M_{\text {scale }}}, & M>M_{\mathrm{c}} \\ 0, & M \leq M_{\mathrm{c}},\end{cases}
$$

where the best-fit values were found to be $M_{\text {scale }} \approx 1.7 M_{\odot}$ and $M_{\mathrm{c}} \approx 6.2 M_{\odot}(\ddot{O}$ zel et al. 2010). An important qualitative feature of the OPNM mass function is the large mass gap between NSs and the lowest-mass BH. Motivated by recent results (Kiziltan et al. 2010) which suggested the mass gap may be less distinct than in Özel et al. (2010), we consider as an alternate case a Gaussian mass function where the best-fit values for mean mass $\mu_{\mathrm{BH}}$ and dispersion $\sigma_{\mathrm{BH}}$ were found to be $7.35 M_{\odot}$ and $1.25 M_{\odot}$, respectively (Farr 


\section{CHAPTER \%. DISK PRECESSION IN SGRBS}

et al. 2011).

Recent observations have measured spins for $7 \mathrm{BHs}$ and placed upper or lower limits on spins for 3 more (McClintock et al. 2011; Gou et al. 2011; Steiner et al. 2012). Although observations of more systems are needed, the current spin distribution is noticeably bimodal. Because of the small number of data points we do not attempt to fit a parametrized spin function, and instead simply take a flat prior on $a_{\mathrm{BH}}$, sampling it uniformly in the ranges $(0,0.3)$ and $(0.7,1)$ for our fiducial, "bimodal" case. For non-fiducial cases, we also consider three alternate spin functions. The "flat", "slow," and "fast" cases uniformly sample $a_{\mathrm{BH}}$ along the intervals $(0,1),(0,0.5)$, and $(0.5,1)$, respectively.

Kicks resulting from asymmetric supernova explosions are expected to produce spin-orbit misalignment in BH-NS binaries. Past research has constrained the allowed pre-merger misalignment angle $\psi$ as a function of progenitor masses and separation, and kick velocity distributions (Kalogera 2000). More recent population synthesis of BH-NS binaries has found a wide spread in pre-merger spin-orbit misalignment $\psi$, but with $\sim 50 \%$ of systems possessing $\psi<45^{\circ}$ (Belczynski et al. 2008). Our fiducial, "prograde" case samples the pre-merger spin-orbit misalignment uniformly in $\psi$ between $0^{\circ}$ and $90^{\circ}$, but we also consider an alternate, "isotropic" case where $\psi$ is sampled uniformly from $0^{\circ}$ to $180^{\circ}$; physically this would represent larger supernova kicks.

We sample NS masses from a Gaussian distribution peaked at a mean $\mu_{\mathrm{NS}}=1.35 M_{\odot}$ with standard deviation $\sigma_{\mathrm{NS}}=0.13 M_{\odot}$. These values, taken from the double NS binaries examined in Ref. (Kiziltan et al. 2010), are in good qualitative agreement with other studies of the NS mass function (Valentim et al. 2011). Because most NS equations of 


\section{CHAPTER \%. DISK PRECESSION IN SGRBS}

state that are not in conflict with observations of $\approx 2 M_{\odot}$ NSs (Demorest et al. 2010) are roughly constant-radius in the relevant mass range, we take a fiducial radius of $13.5 \mathrm{~km}$, but as an alternate case consider a NS radius of $11 \mathrm{~km}$.

With these distributions defined, we are now ready to populate a large Monte Carlo sample of BH-NS mergers. Our precise procedure is as follows, for any desired set of distributions:

1. Generate $2 \times 10^{5} \mathrm{BH}$ masses $M_{\mathrm{BH}}$, spin magnitudes $a_{\mathrm{BH}}$, initial misalignment angles $\psi$, and NS masses $M_{\mathrm{NS}}$.

2. Compute the pre-merger $r_{\text {ISSO }}$ from Eq. (C.3).

3. Calculate the post-merger $\mathrm{BH}$ mass $M_{\mathrm{BH}}^{\prime}, \mathrm{BH}$ spin $a_{\mathrm{BH}}^{\prime}$, and spin-disk misalignment $\psi_{\mathrm{d}}$ using Eqs. (D.2) and (D.3).

4. Flag the disruption as GRB-producing if $M_{\mathrm{d}} / M_{\mathrm{NS}}>0.05$.

5. Compute $N_{\text {cycles }}, T_{\text {prec }}\left(t_{1 / 2}\right)$, and $f_{\mathrm{GRB}}$ (using the post-merger $r_{\mathrm{ISSO}}^{\prime}$ ). 


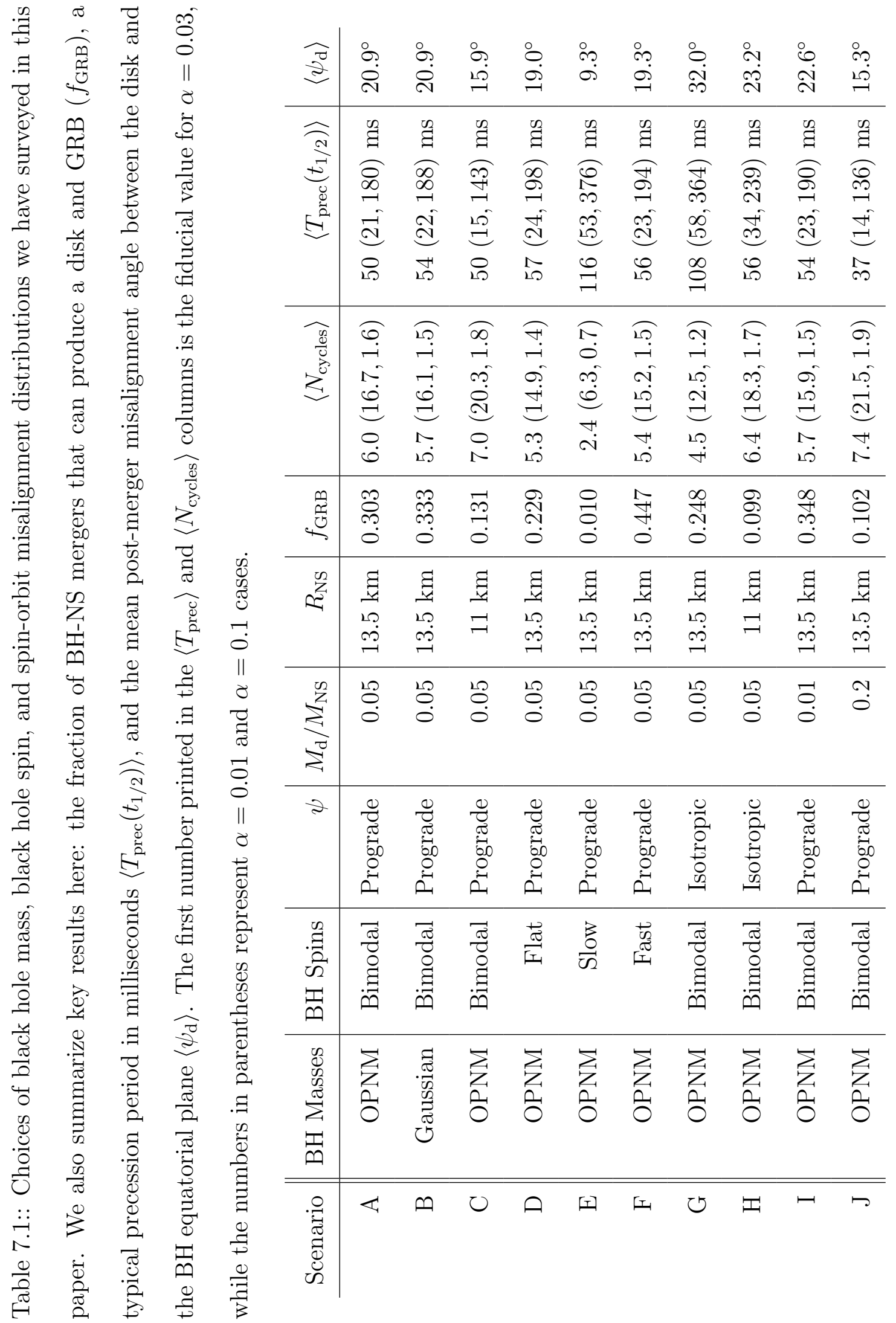



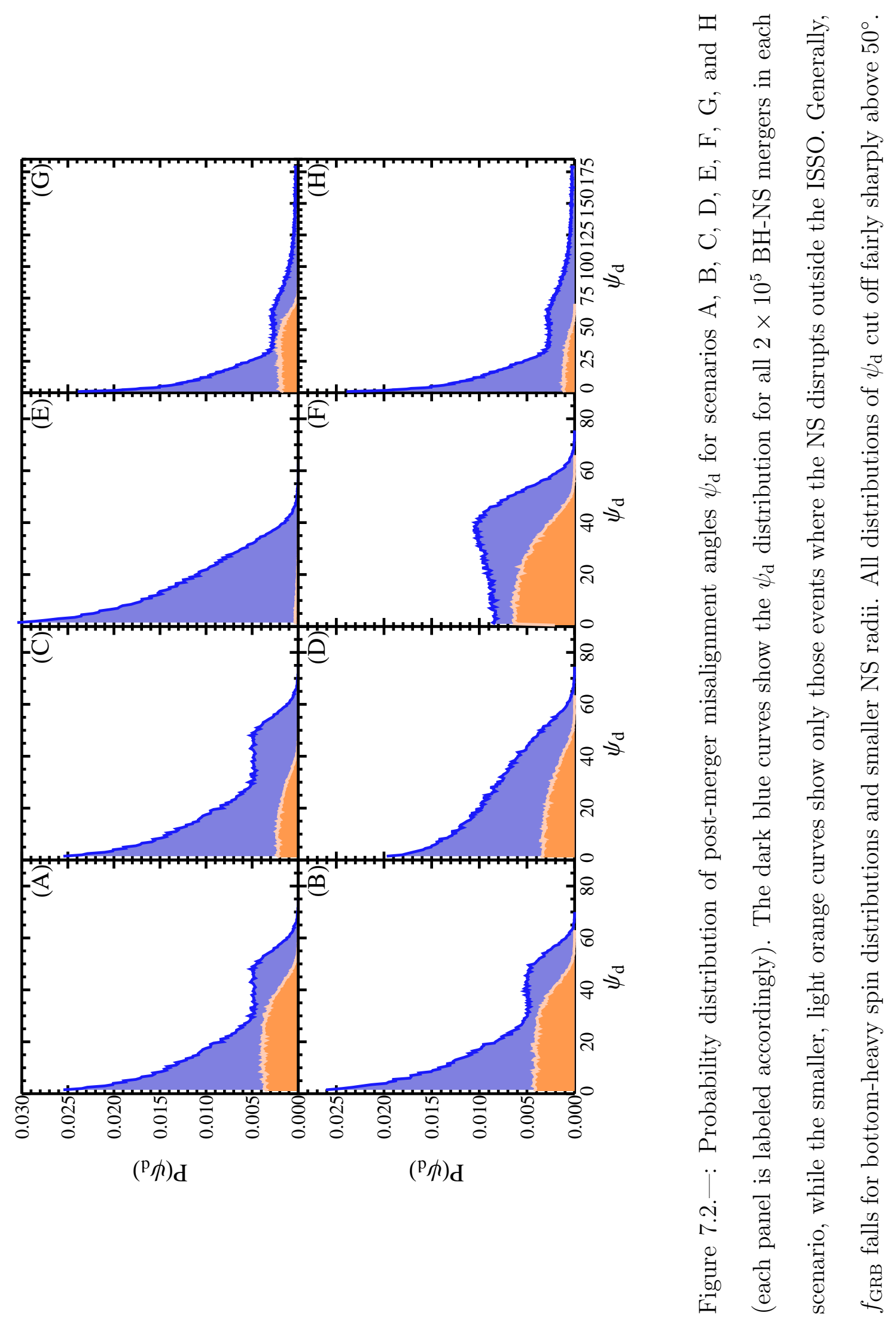


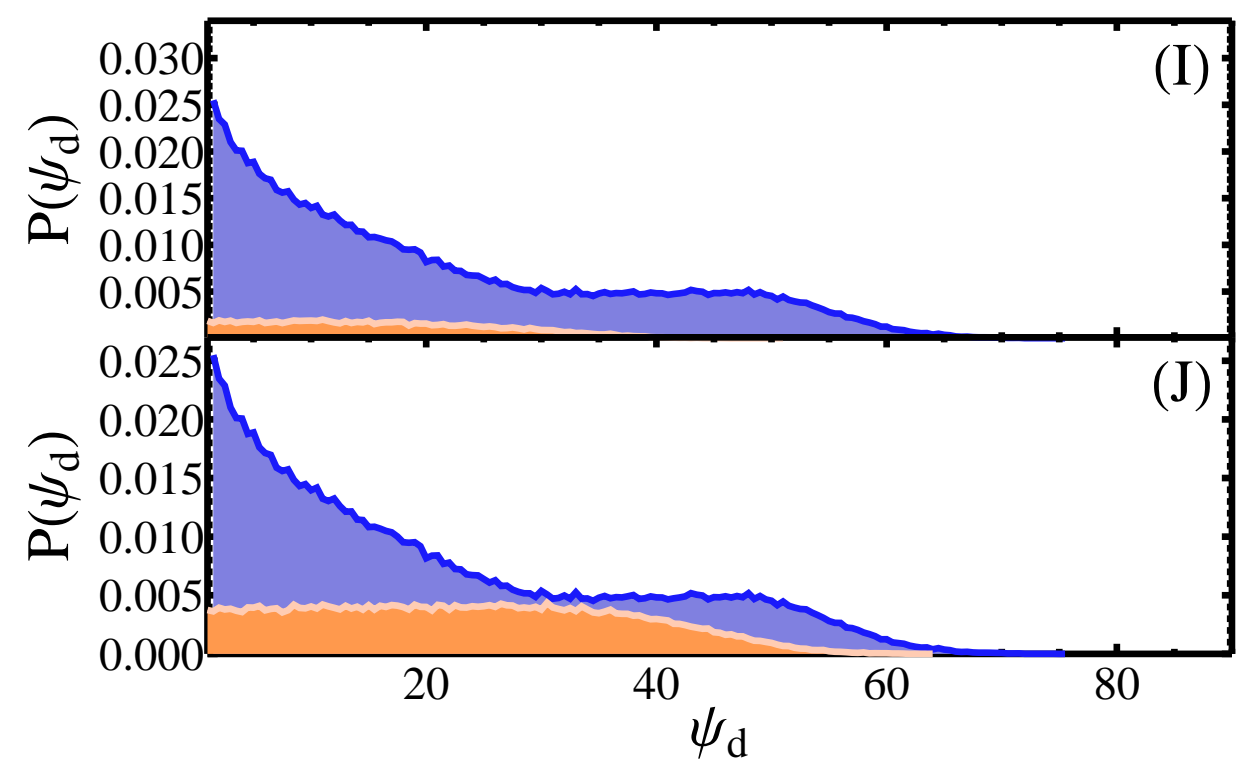

Figure 7.3.- : The probability distribution of post-merger misalignment angles $\psi_{\mathrm{d}}$ for scenarios I and $\mathrm{J}$, illustrating the dependence of $f_{\mathrm{GRB}}$ and $\psi_{\mathrm{d}}$ on the strictness of our requirement for $M_{\mathrm{d}} / M_{\mathrm{NS}}$. Specifically, in the top panel we have imposed the strict requirement that $M_{\mathrm{d}} / M_{\mathrm{NS}}>0.2$ in order to produce a SGRB, while in the lower panel we have imposed the much laxer requirement of $M_{\mathrm{d}} / M_{\mathrm{NS}}>0.01$. In all other respects both of these cases are identical to scenario A. Results in the bottom panel are quite similar to scenario A, but in the top panel, $f_{\mathrm{GRB}}$ has been strongly suppressed, particularly at higher $\psi_{\mathrm{d}}$. The axes and curves are the same as in Fig. 7.2. 

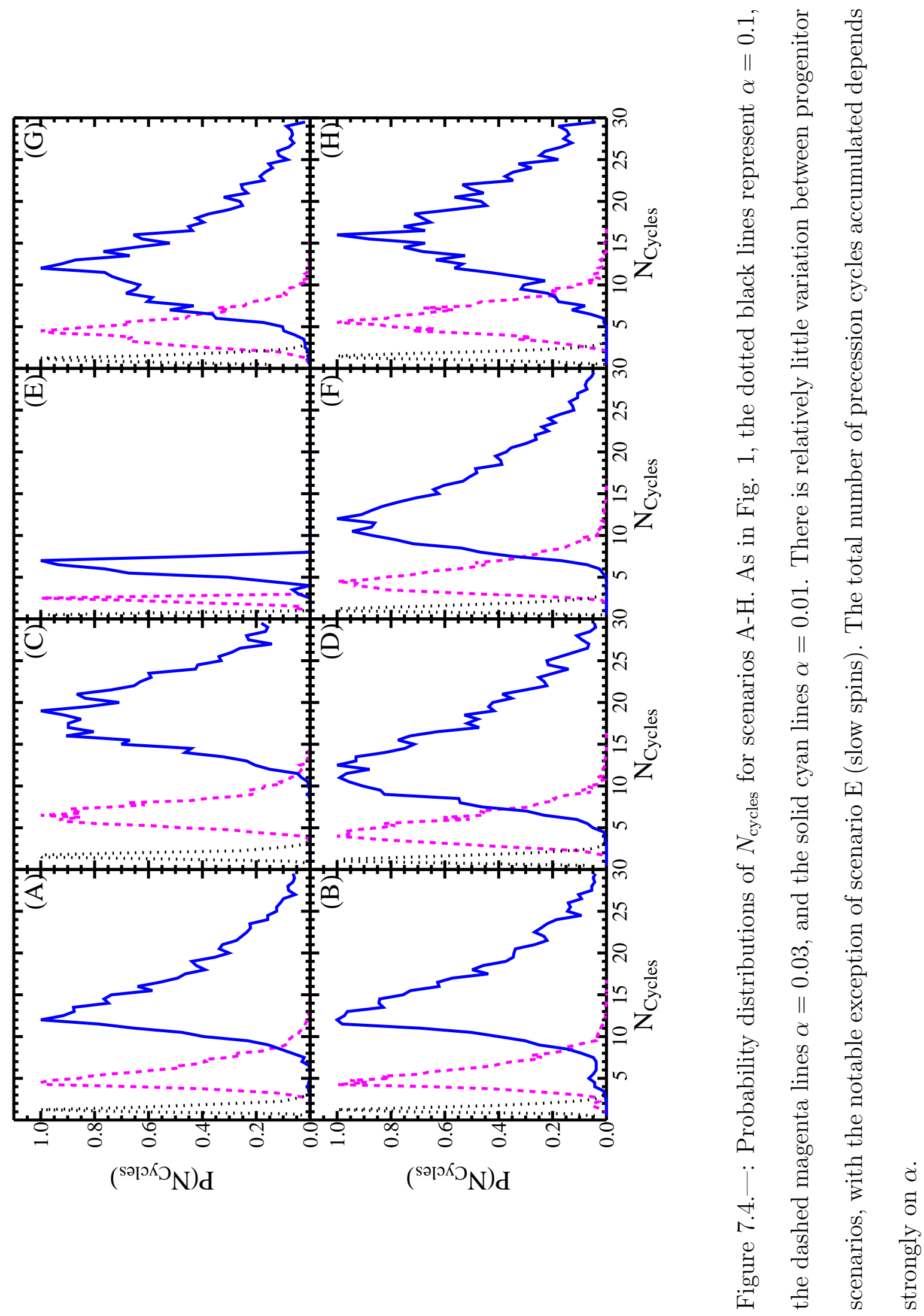

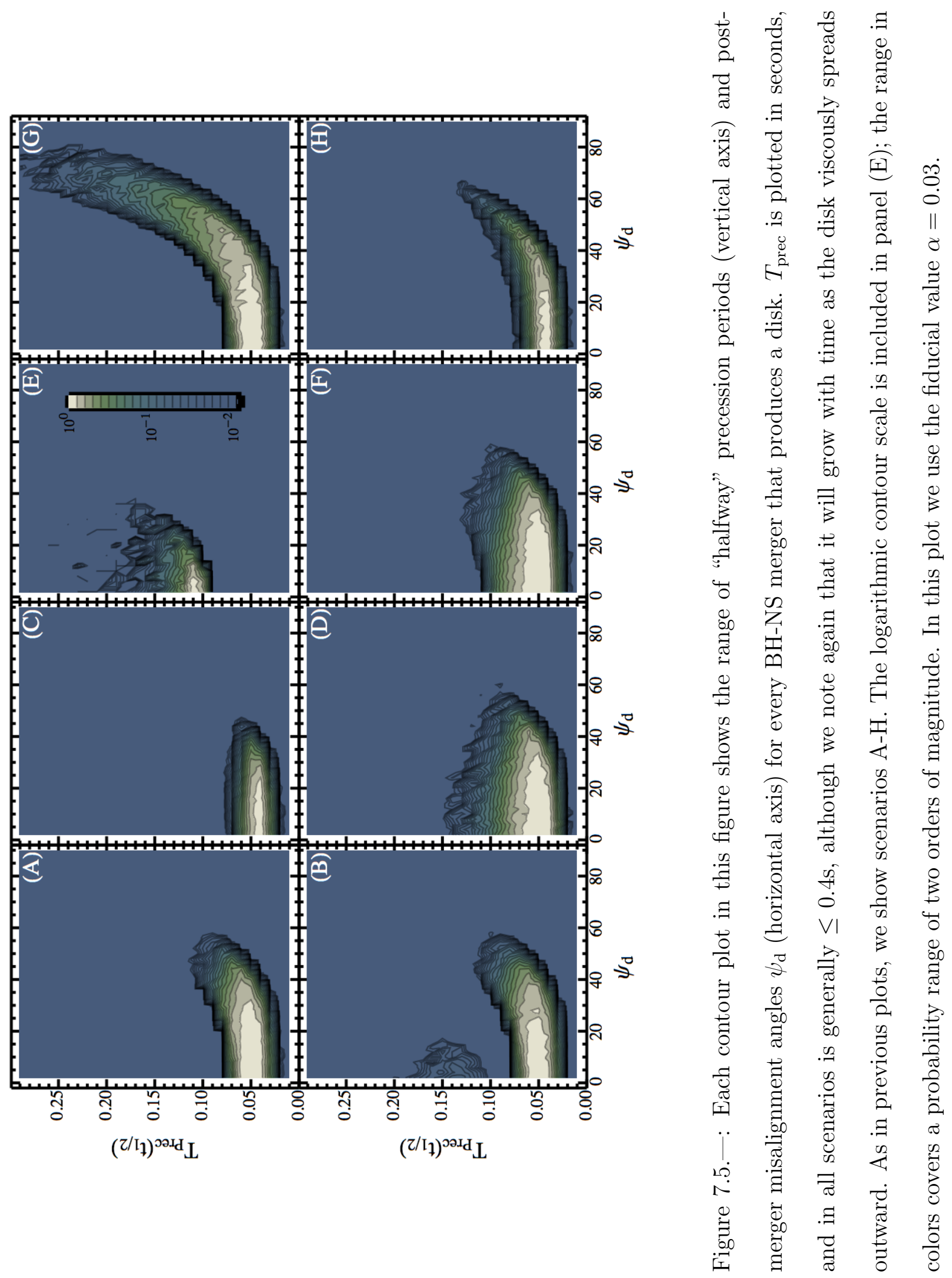


\section{CHAPTER \%. DISK PRECESSION IN SGRBS}

In Table I, we summarize the scenarios considered in this work, along with averaged results. Typical precession periods are $\left\langle T_{\text {prec }}\left(t_{1 / 2}\right)\right\rangle \approx 50 \mathrm{~ms}-100 \mathrm{~ms}$, with mean disk-BH misalignment typically $\left\langle\psi_{\mathrm{d}}\right\rangle \approx 20^{\circ}$. These results are generally insensitive to variation of assumptions about the progenitor population. The fraction of BH-NS mergers which produce SGRBs $\left(f_{\mathrm{GRB}}\right)$ varies more with the properties of progenitor binaries, and in particular depends strongly on the value of pre-merger BH spin $a_{\mathrm{BH}}$. Values of $a_{\mathrm{BH}} \gtrsim 0.5$ are generally necessary to produce a post-merger disk. Scenario A, our fiducial case, results in GRBs for $\approx 30 \%$ of BH-NS mergers. Results for scenario A are quite similar to those in scenarios B (Gaussian BH mass function) and D (flat prior on the BH spin distribution). The probability of disruption of the NS falls by a factor $\approx 2$ in scenario $\mathrm{C}$ (softer NS equation of state), and becomes almost negligible in scenario E (bottom-heavy spin distribution). The fraction of BH-NS mergers capable of producing SGRBs is maximized in scenario $\mathrm{F}$ (fast spins), reduced somewhat if we switch to an isotropic $\psi$ distribution (scenario G), and reduced significantly for a stricter lower cutoff on $M_{\mathrm{d}} / M_{\mathrm{NS}}$ (scenario J). Our fiducial scenario comes close to maximizing $f_{\mathrm{GRB}}$; only switching over to top-heavy BH spin distributions (scenario F) or laxer SGRB criteria (scenario I) increase its value. More detail on $f_{\mathrm{GRB}}$ and distributions of $\psi_{\mathrm{d}}$ can be seen in Figure 7.2.

In Figure 7.3, we explore the sensitivity of $f_{\mathrm{GRB}}$ and $\psi_{\mathrm{d}}$ to our uncertain assumption about the minimum normalized disk mass, $M_{\mathrm{d}} / M_{\mathrm{NS}}$, needed to produce a SGRB. In our standard scenarios, we take a cutoff value of 0.05 , but in scenarios I and $\mathrm{J}$ we change this cutoff to 0.01 and 0.2 , respectively. While relaxing the cutoff seems to have little effect on our overall results, increasing the cutoff above $M_{\mathrm{d}} / M_{\mathrm{NS}} \approx 0.1$ very quickly suppresses $f_{\mathrm{GRB}}$, and biases those SGRBs which are produced toward low-tilt accretion disks.

In general, $\psi_{\mathrm{d}}$ is strongly cut off above $40^{\circ}-50^{\circ}$ in all scenarios except $\mathrm{E}$, and 


\section{CHAPTER \%. DISK PRECESSION IN SGRBS}

there is little variation between the mean value $\left\langle\psi_{\mathrm{d}}\right\rangle$ in each of our progenitor scenarios. Specifically, $\left\langle\psi_{\mathrm{d}}\right\rangle$ ranges from $9^{\circ}$ to $32^{\circ}$. Interestingly, our two scenarios with isotropic pre-merger $\psi$ only modestly extend the range of post-merger $\psi_{\mathrm{d}}$ values: it seems that strongly misaligned $\left(\psi>90^{\circ}\right)$ BH-NS mergers simply do not produce massive disks. As mentioned earlier, our calculation of $\psi_{\mathrm{d}}$ likely overestimates its true value by a factor $\approx 2$, because the tilt angle will decrease as the disk settles into equilibrium, so the true range of $\left\langle\psi_{\mathrm{d}}\right\rangle$ would more accurately be $\sim 5^{\circ}$ to $\sim 15^{\circ}$. For rapidly spinning BHs, it is possible that our $\psi_{\mathrm{d}}$ estimate accurately describes the first few precession cycles, before disk tilt has time to decrease.

As can be seen in Table I, the key observables $\left\langle N_{\text {cycles }}\right\rangle$ and $\left\langle T_{\text {prec }}\left(t_{1 / 2}\right)\right\rangle$ depend much more sensitively on $\alpha$ than they do on the parameters of BH-NS binary populations. As usual, the outlier is scenario E, which is biased toward few cycles and long precession timescales, but all other scenarios produce $4.5 \lesssim N_{\text {cycles }} \lesssim 8$ and $30 \mathrm{~ms} \lesssim\left\langle T_{\text {prec }}\left(t_{1 / 2}\right)\right\rangle \lesssim 65 \mathrm{~ms}$, for our fiducial $\alpha=0.03$ case. We plot distributions of $\left\langle N_{\text {cycles }}\right\rangle$ in Fig. 7.4, where we see that low $\alpha$ values produce many more disk precession cycles.

In Figure 7.5 we plot contours to indicate the relative probability of BH-NS mergers producing combinations of $T_{\mathrm{prec}}$ and $\psi_{\mathrm{d}}$. These two quantities in general appear fairly uncorrelated, although in some scenarios (A, B and G) we do see a weak positive correlation, indicating that the most dramatically precessing SGRB disks will typical precess with longer periods. In no scenario do we see typical $T_{\text {prec }}\left(t_{1 / 2}\right)$ values above 0.2 $\mathrm{s}$; if we set aside scenario $\mathrm{E}$, only a small fraction of events have $T_{\text {prec }}\left(t_{1 / 2}\right)$ above 0.1 s. Scenarios C and H (soft NS equation of state) generally have the shortest precession periods, as a combination of rapidly spinning $\mathrm{BHs}$ and fairly low $\psi$ are required to 
CHAPTER \%. DISK PRECESSION IN SGRBS

disrupt these more compact NSs. Generally, $T_{\text {prec }}\left(t_{1 / 2}\right) \gtrsim 0.01 \mathrm{~s}$ in all scenarios.

\subsection{Discussion}

In this paper, we have explored much of the relevant parameter space for BH-NS mergers, and found that quantities relevant for disk precession are generally insensitive to the assumptions we have made about progenitor binary parameters. The important results of our calculations $\left(\left\langle T_{\text {prec }}\left(t_{1 / 2}\right)\right\rangle,\left\langle N_{\text {cycles }}\right\rangle,\left\langle\psi_{\mathrm{d}}\right\rangle, f_{\mathrm{GRB}}\right)$ typically change by factors $\lesssim 2$ as we have varied our assumptions about the underlying populations of pre-merger BH-NS binaries. The one exception to this concerns pre-merger BH spin: in order for a significant fraction of BH-NS mergers to produce SGRBs, $a_{\mathrm{BH}} \gtrsim 0.5$ is required. Because of the difficulty of increasing $\mathrm{BH}$ spin through pre-merger mass transfer (Belczynski et al. 2008), this is equivalent to requiring modestly large natal $a_{\mathrm{BH}}$ values. The strongest precession effects (i.e. large misalignment $\psi_{\mathrm{d}}$ and short period $T_{\text {prec }}$ ) arise from populations with top-heavy BH spin distributions and stiff NS equations of state. Our results are notably insensitive to choice of $\mathrm{BH}$ mass function, so long as the mass function peaks near $6 M_{\odot}$, as is suggested by observations of X-ray binaries.

On the other hand, our results do depend fairly sensitively on the details of how the disk will viscously spread outward. Using the $\alpha$ viscosity parametrization, we have seen that effective $\alpha$ values $\gtrsim 0.1$ will strongly suppress the number of observable precession cycles, to the point where jet precession will rarely if ever be detectable. Likewise,

$\alpha \gtrsim 0.1$ could be large enough to induce a Bardeen-Petterson warp in the thinner, inner regions of the disk, limiting global precession. We note again, however, that what matters most for the time evolution of $T_{\text {prec }}$ is not the value of $\alpha$ in the inner regions of the 


\section{CHAPTER \%. DISK PRECESSION IN SGRBS}

disk, where GR effects enhance the turbulent viscosity produced by the MRI (McKinney \& Narayan 2007), but rather the outer regions of the disk, where local shearing box simulations that find lower $(0.01 \lesssim \alpha \lesssim 0.1)$ levels of MRI-generated viscosity are more appropriate (Davis et al. 2010).

If jets align with the disk angular momentum axis, then they will precess around the total angular momentum vector by an angle $\approx \psi_{\mathrm{d}}$, because $J_{\mathrm{BH}}$ is significantly larger than $J_{\text {disk }}$. In this case, observations of SGRBs associated with BH-NSs will often be marked by a clear "lighthouse effect," so long as $\psi_{\mathrm{d}} \gtrsim \theta_{\text {jet }}$. This seems plausible, as observations of jet breaks in SGRBs suggest typical opening angles of $\sim 10^{\circ}$ (Soderberg et al. 2006). If $\psi_{\mathrm{d}} \lesssim \theta_{\text {jet }}$, then jet precession would, typically, be encoded more subtly as a variation in the portion of the jet presented to the observer. Alternatively, if jets align with the $\mathrm{BH}$ spin axis, they will precess by a much smaller amount, since the angle between the $\mathrm{BH}$ spin vector and the total system angular momentum vector is $\cos \psi_{\mathrm{BH}}=\left(1+j \cos \psi_{\mathrm{d}}\right) / \sqrt{1+2 j \cos \psi_{\mathrm{d}}+j^{2}}$, where $j=J_{\mathrm{d}} / J_{\mathrm{BH}}$. This angle will initially be smaller than $\psi_{\mathrm{d}}$ by a factor $\sim 10$ if $J_{\mathrm{d}} \sim 0.1 J_{\mathrm{BH}}$ (Foucart et al. 2011), and this difference will increase slowly in time as angular momentum is accreted from the disk onto the $\mathrm{BH}$ (or quickly, if angular momentum is lost in outflows). Unless the typical $\theta_{\text {jet }}$ is quite small, less than a few degrees, the dramatic lighthousing effect previously discussed would be unlikely. A quasi-periodic signal will still be present in all these scenarios, but it will be strongest for tightly collimated jets aligned with $\vec{J}_{\text {disk }}$. When we correct our results for the $\approx 2$ overestimate in $\psi_{\mathrm{d}}$, we have found $\left\langle\psi_{\mathrm{d}}\right\rangle \sim \theta_{\text {jet }}$, although there are substantial observational uncertainties regarding the distribution of jet opening angles.

Detection of a precession signal appears observationally feasible: our study found a 


\section{CHAPTER \%. DISK PRECESSION IN SGRBS}

floor of $\sim 25 \mathrm{~ms}$ for the "halfway" precession period $\left\langle T_{\text {prec }}\left(t_{1 / 2}\right)\right\rangle$, but precession periods of $\sim 50$ msec were much more common, and in all cases the precession period grows in time. Both Swift and Fermi GBM have much finer intrinsic timing resolution, so the observability of precession signals will be ultimately limited by photon-counting statistics. Analysis of Fermi SGRB light curves finds evidence of structure in the $\sim 10-50 \mathrm{msec}$ range (Bhat et al. 2012), while Swift BAT also produces signals with structure in the 10s of msec (Rowlinson et al. 2010). For comparison, the BATSE archival data sample contains sufficiently high-resolution timing data to enable a search for $\sim \mathrm{kHz}$ frequency QPOs (Preece et al. 2000; Kruger et al. 2002), indicating that the much longer precession signals should be clearly resolvable.

The two primary challenges for observability are (1) the relatively low number of precession cycles, and (2) the rapidly growing precession period $T_{\text {prec }}$. However, we have shown that for most progenitor populations there exists a large-value tail to the $N_{\text {cycles }}$ distribution, from which events with $N_{\text {cycles }} \approx 5$ can be observed, provided $\alpha<0.1$. Furthermore, searches for evolving quasiperiodicity are feasible, provided the time axis of time series data can be rescaled to match appropriate theoretical models. In this paper we have employed a simple analytic model for the viscous spreading of the disk, and for the first time have found that precession periods grow as $t^{4 / 3}$. We hope that this will provide a starting point for searches for jet precession in SGRBs, but detailed hydrodynamical, and perhaps GRMHD, simulations are necessary to validate or refine this analytic expectation. We also note that both of these challenges are mitigated if a significant fraction of disk angular momentum is lost through an outflow, which will reduce the late-time scaling of $T_{\text {prec }}$ to $t^{4 / 5}$.

Pulsation of prompt emission may not be the only observable implication of disk 


\section{CHAPTER \%. DISK PRECESSION IN SGRBS}

precession in SGRBs. A precessing jet will sweep out a larger solid angle in the sky, enabling BH-NS mergers to make up a larger fraction of the observed SGRB rate than would be implied by a simple calculation (i.e. the intrinsic BH-NS merger rate multiplied by $\left.f_{\text {GRB }}\right)$. This would likewise enhance observability of BH-NS optical afterglows (Metzger \& Berger 2012).

Unless there exists a large population of NS-NS binaries with millisecond spin periods (not accounted for by current population synthesis estimates), SGRBs due to BH-NS mergers will be distinguishable from those due to NS-NS mergers by the presence of a quasiperiodic signal, with a typical period of $\sim 30-100 \mathrm{~ms}$. We have shown that this signal is robust to a large number of assumptions about the progenitor binaries, but evolves quickly and could become difficult to observe if jets align with the BH spin axis, or if the post-merger disk viscosity is large. A better understanding of both viscous disk spreading, and how GRB jet intensity varies with angle of observation, will aid future searches for this discriminant between SGRB progenitor binaries. 


\section{Chapter 8}

\section{Conclusions and Future Directions}

The tidal disruption of stars by supermassive black holes (SMBHs) is an exciting research frontier in both theoretical and observational astronomy. In this thesis, we have studied these dramatic events from a theoretical perspective and found that they hold great promise: as measures of SMBH demography; as probes to study strong-field general relativity; and as laboratories for high energy accretion physics. For many decades tidal disruption events (TDEs) were a theory-dominated subject with no substantiating observations. Fortunately, we now live in an era where exciting new TDE detections are reported every year, and increasingly theorists' models are challenged by unexpected observations.

In Chapters 2 and 3, we investigated how TDEs can be used as indicators of SMBH recoil, a prediction of relatively recent numerical relativity breakthroughs. Immediately following the coalescence of a SMBH binary, there will be a burst of TDEs as the host galaxy's "loss cone" refills due to gravitational wave recoil. In a relatively conservative dry merger scenario, this burst produces one TDE every few decades for typical recoils 


\section{CHAPTER 8. CONCLUSIONS AND FUTURE DIRECTIONS}

among LISA-band $\left(\sim 10^{6} M_{\odot}\right)$ black holes. However, this rate could be enhanced by resonant migration of stars in dry mergers, or viscous migration of stars in wet mergers. The first of these possibilities could perhaps be studied analytically, but the second likely requires numerical simulation. If either mechanism proves viable, it would provide a strong electromagnetic counterpart for future low-frequency gravitational wave astronomy. In the nearer term, the $L S S T$ will go online within a decade, and should be able to detect spatially offset tidal disruption flares at a rate of $\sim 1-10 \mathrm{yr}^{-1}$. Although a small minority of the total TDE population, such offset flares would serve as valuable tracers of SMBH recoil.

In Chapter 4, we argued that traditional estimates for TDE mass fallback timescales were only correct for grazing, marginal disruptions, and are in fact enormously incorrect for deeply plunging disruptions. The vertical collapse and bounce of a tidally disrupting star, general relativity, and stellar spin all seem inadequate on their own to change this conclusion, although it is possible that combinations of them might restore some $\beta$ dependence to the dynamical outcome of a TDE. Future work should address whether misaligned SMBH spin is capable of desynchronizing a bounce strongly enough to change the spread in debris energy. The distribution of $\beta$ within a sample of TDEs carries valuable information about stellar dynamical processes, and the onus is now on theorists to discover novel ways to measure it. One speculative possibility that we have raised for the first time is high frequency gravitational wave emission, which can be generated by the rapidly changing internal quadrupole moment of a tidally "bouncing" star. This is a scenario that can be studied in more detail with full numerical simulations, or analytically with the parabolic free solutions we have used here.

In Chapter 5, we have examined a new subset of TDEs: those on eccentric, rather 


\section{CHAPTER 8. CONCLUSIONS AND FUTURE DIRECTIONS}

than parabolic, orbits. While these are likely subdominant in terms of rates, their initial stages of accretion are enormously super-Eddington, which may make them visible to larger distances. We have also used our hydrodynamical simulations of these events to study a particularly difficult problem in TDE physics: debris circularization. For significantly eccentric TDEs, the debris circularizes through stream-stream collisions that are driven by general relativistic precession. Determining whether or not this mechanism applies to the more common parabolic disruptions is an important challenge for future theoretical work. If it does, then it is likely that the spins of SMBHs will be imprinted on circularization timescales, offering an indirect way for TDEs to constrain SMBH spin.

In Chapter 6, we argued that TDE flares offer a more direct way to measure SMBH spin: direct precession of the accretion disk, driven by Lense-Thirring torques. A precessing TDE disk will exhibit quasiperiodic oscillations in its light curve and spectrum as different portions of the disk are presented to the observer, potentially enabling a direct SMBH spin measurement. In the future, our simple model for precession could be coupled to more complete (semi)analytic models for time-dependent disk evolution, or compared directly to numerical simulations. We have tentatively used our model to address an open question in accretion physics (alignment of jets in tilted accretion flows), and argued that in the Swift $1644+57$ flare, the jet likely aligned with the SMBH spin vector. Other "one-off" possibilities are improbable but viable, however, and a larger sample of relativistic TDE flares will be needed to settle the question of jet alignment.

Finally, in Chapter 7, we have applied our work in Chapter 6 to an analogous scenario: the transient accretion disk formed by the mergers of neutron stars with other compact objects. Only in the case of a neutron star-black hole merger (and not in a double neutron star scenario) would disk and jet precession occur, possibly offering a 
way to distinguish between two viable progenitors of short gamma ray bursts (SGRBs). In practice, a significant number of precession cycles can accumulate if the disk viscosity is low, but a high viscosity will quench any precession signal. Our simple analytic calculations have let us survey parameter space widely, but the next step is combining them with numerical one- or two-dimensional disk models.

In conducting the research outlined here, we have often come across as many new questions as we have answered old ones. In this concluding section I have given an overview of my past research, as well as some brief thoughts on promising future research directions. Of course, progress will come from many different avenues. The advent of time domain optical astronomy has already revolutionized many fields, and it may do the same for TDE physics. However, realizing the full scientific promise of TDE observations requires more detailed theoretical models, so that we can extract the wealth of information these events likely carry on SMBH demographics, general relativity, and extreme accretion environments. 


\section{References}

Alexander, R. D., Armitage, P. J., \& Cuadra, J. 2008, MNRAS, 389, 1655

Alexander, T., \& Morris, M. 2003, ApJ, 590, L25

Amaro-Seoane, P., Gair, J. R., Freitag, M., Miller, M. C., Mandel, I., Cutler, C. J., \& Babak, S. 2007, Classical and Quantum Gravity, 24, 113

Amaro-Seoane, P., Miller, M. C., \& Kennedy, G. F. 2012a, MNRAS, 425, 2401

Amaro-Seoane, P., Sopuerta, C. F., \& Freitag, M. D. 2013, MNRAS, 429, 3155

Amaro-Seoane, P., et al. 2012b, Classical and Quantum Gravity, 29, 124016

Anninos, P., Fragile, P. C., Wilson, J., \& Murray, S. D. 2012, ApJ, 759, 132

Arun, K. G., et al. 2009, Classical and Quantum Gravity, 26, 094027

Ayal, S., Livio, M., \& Piran, T. 2000, ApJ, 545, 772

Bade, N., Komossa, S., \& Dahlem, M. 1996, A\&A, 309, L35

Baganoff, F. K., et al. 2003, ApJ, 591, 891

Bahcall, J. N., \& Wolf, R. A. 1976, ApJ, 209, 214

Baker, J. G., Centrella, J., Choi, D.-I., Koppitz, M., \& van Meter, J. 2006, Physical Review Letters, 96, 111102

Bandara, K., Crampton, D., \& Simard, L. 2009, ApJ, 704, 1135

Bar-Or, B., Kupi, G., \& Alexander, T. 2013, ApJ, 764, 52

Bardeen, J. M., \& Petterson, J. A. 1975, ApJ, 195, L65

Bardeen, J. M., Press, W. H., \& Teukolsky, S. A. 1972, ApJ, 178, 347 


\section{REFERENCES}

Bartko, H., et al. 2010, ApJ, 708, 834

Bate, M. 1995, PhD thesis, PhD thesis, Univ. Cambridge, (1995)

Bate, M. R., Bonnell, I. A., \& Price, N. M. 1995, MNRAS, 277, 362

Beckmann, V., \& Shrader, C. R. 2012, Active Galactic Nuclei

Begelman, M. C., Rossi, E. M., \& Armitage, P. J. 2008, MNRAS, 387, 1649

Begelman, M. C., Volonteri, M., \& Rees, M. J. 2006, MNRAS, 370, 289

Belczynski, K., Taam, R. E., Rantsiou, E., \& van der Sluys, M. 2008, ApJ, 682, 474

Beloborodov, A. M., Illarionov, A. F., Ivanov, P. B., \& Polnarev, A. G. 1992, MNRAS, 259, 209

Bentz, M. C., Peterson, B. M., Pogge, R. W., \& Vestergaard, M. 2009, ApJ, 694, L166

Benz, W., Cameron, A. G. W., Press, W. H., \& Bowers, R. L. 1990, ApJ, 348, 647

Berger, E. 2009, ApJ, 690, 231

-. 2010, ApJ, 722, 1946

Berger, E., et al. 2005, Nature, 438, 988

—. 2007, ApJ, 664, 1000

Berti, E., \& Volonteri, M. 2008, ApJ, 684, 822

Bhat, P. N., et al. 2012, ApJ, 744, 141

Bicknell, G. V., \& Gingold, R. A. 1983, ApJ, 273, 749

Biermann, P. L., \& Strittmatter, P. A. 1987, ApJ, 322, 643

Binney, J., \& Tremaine, S. 2008, Galactic Dynamics: Second Edition (Princeton University Press)

Blackman, E. G., Yi, I., \& Field, G. B. 1996, ApJ, 473, L79

Blanchet, L. 2006, Living Reviews in Relativity, 9, 4

Blandford, R. D., \& Znajek, R. L. 1977, MNRAS, 179, 433 


\section{REFERENCES}

Blecha, L., Cox, T. J., Loeb, A., \& Hernquist, L. 2011, MNRAS, 412, 2154

Blecha, L., \& Loeb, A. 2008, MNRAS, 390, 1311

Bloom, J. S., et al. 2006, ApJ, 638, 354

—. 2011, Science, 333, 203

Bode, T., Haas, R., Bogdanović, T., Laguna, P., \& Shoemaker, D. 2010, ApJ, 715, 1117

Bogdanović, T., Eracleous, M., Mahadevan, S., Sigurdsson, S., \& Laguna, P. 2004, ApJ, 610, 707

Bogdanović, T., Eracleous, M., \& Sigurdsson, S. 2009, ApJ, 697, 288

Bogdanović, T., Reynolds, C. S., \& Miller, M. C. 2007, ApJ, 661, L147

Bonning, E. W., Shields, G. A., \& Salviander, S. 2007, ApJ, 666, L13

Bower, G. C., Metzger, B. D., Cenko, S. B., Silverman, J. M., \& Bloom, J. S. 2013, ApJ, 763, 84

Brandt, W. N., Pounds, K. A., \& Fink, H. 1995, MNRAS, 273, L47

Brassart, M., \& Luminet, J.-P. 2008, A\&A, 481, 259

-. 2010, A\&A, 511, A80

Brem, P., Amaro-Seoane, P., \& Sopuerta, C. F. 2012, ArXiv e-prints

Brenneman, L. W., \& Reynolds, C. S. 2006, ApJ, 652, 1028

Broderick, A. E., \& Loeb, A. 2005, MNRAS, 363, 353

-. 2009, ApJ, 697, 1164

Broderick, A. E., Loeb, A., \& Narayan, R. 2009, ApJ, 701, 1357

Bromley, B. C., Kenyon, S. J., Geller, M. J., \& Brown, W. R. 2012, ApJ, 749, L42

Bromm, V., \& Loeb, A. 2003, ApJ, 596, 34

Burkert, A., Schartmann, M., Alig, C., Gillessen, S., Genzel, R., Fritz, T. K., \& Eisenhauer, F. 2012, ApJ, 750, 58

Burkert, A., \& Tremaine, S. 2010, ApJ, 720, 516 


\section{REFERENCES}

Burrows, D. N., et al. 2006, ApJ, 653, 468

-. 2011, Nature, 476, 421

Byun, Y.-I., et al. 1996, AJ, 111, 1889

Callegari, S., Mayer, L., Kazantzidis, S., Colpi, M., Governato, F., Quinn, T., \& Wadsley, J. 2009, ApJ, 696, L89

Campanelli, M., Lousto, C., Zlochower, Y., \& Merritt, D. 2007, ApJ, 659, L5

Campanelli, M., Lousto, C. O., Marronetti, P., \& Zlochower, Y. 2006, Physical Review Letters, 96, 111101

Cannizzo, J. K., Lee, H. M., \& Goodman, J. 1990, ApJ, 351, 38

Carter, B., \& Luminet, J. P. 1982, Nature, 296, 211

Carter, B., \& Luminet, J.-P. 1983, A\&A, 121, 97

Carter, B., \& Luminet, J. P. 1985, MNRAS, 212, 23

Cenko, S. B., et al. 2012a, MNRAS, 420, 2684

-. 2012b, ApJ, 753, 77

Chen, W.-X., \& Beloborodov, A. M. 2007, ApJ, 657, 383

Chen, X., Madau, P., Sesana, A., \& Liu, F. K. 2009, ApJ, 697, L149

Chen, X., Sesana, A., Madau, P., \& Liu, F. K. 2011, ApJ, 729, 13

Civano, F., et al. 2010, ApJ, 717, 209

Cohn, H., \& Kulsrud, R. M. 1978, ApJ, 226, 1087

Colpi, M., \& Dotti, M. 2009, ArXiv e-prints

Comerford, J. M., et al. 2009, ApJ, 698, 956

Dai, L., \& Blandford, R. D. 2011, ArXiv e-prints

Davies, M. B., Miller, M. C., \& Bellovary, J. M. 2011, ApJ, 740, L42

Davis, S. W., Stone, J. M., \& Pessah, M. E. 2010, ApJ, 713, 52

De Colle, F., Guillochon, J., Naiman, J., \& Ramirez-Ruiz, E. 2012, ApJ, 760, 103 


\section{REFERENCES}

Demorest, P. B., Pennucci, T., Ransom, S. M., Roberts, M. S. E., \& Hessels, J. W. T. 2010, Nature, 467, 1081

Devecchi, B., \& Volonteri, M. 2009, ApJ, 694, 302

Devecchi, B., Volonteri, M., Colpi, M., \& Haardt, F. 2010, MNRAS, 409, 1057

Devecchi, B., Volonteri, M., Rossi, E. M., Colpi, M., \& Portegies Zwart, S. 2012, MNRAS, 421, 1465

Dexter, J., \& Fragile, P. C. 2011, ApJ, 730, 36

Di Matteo, T., Springel, V., \& Hernquist, L. 2005, Nature, 433, 604

Diener, P., Frolov, V. P., Khokhlov, A. M., Novikov, I. D., \& Pethick, C. J. 1997, ApJ, 479, 164

Diener, P., Kosovichev, A. G., Kotok, E. V., Novikov, I. D., \& Pethick, C. J. 1995, MNRAS, 275, 498

Doeleman, S. S., Fish, V. L., Broderick, A. E., Loeb, A., \& Rogers, A. E. E. 2009, ApJ, 695, 59

Donley, J. L., Brandt, W. N., Eracleous, M., \& Boller, T. 2002, AJ, 124, 1308

Dotti, M., Volonteri, M., Perego, A., Colpi, M., Ruszkowski, M., \& Haardt, F. 2010, MNRAS, 402, 682

Drasco, S., \& Hughes, S. A. 2004, Phys. Rev. D, 69, 044015

Escala, A., Larson, R. B., Coppi, P. S., \& Mardones, D. 2004, ApJ, 607, 765

—. 2005, ApJ, 630, 152

Esquej, P., Saxton, R. D., Freyberg, M. J., Read, A. M., Altieri, B., Sanchez-Portal, M., \& Hasinger, G. 2007, A\&A, 462, L49

Esquej, P., et al. 2008, A\&A, 489, 543

Evans, C. R., \& Kochanek, C. S. 1989, ApJ, 346, L13

Faber, S. M., et al. 1997, AJ, 114, 1771

Fan, X., et al. 2003, AJ, 125, 1649

Fan, Y.-Z., \& Wei, D.-M. 2011, ApJ, 739, 47 


\section{REFERENCES}

Farr, W. M., Sravan, N., Cantrell, A., Kreidberg, L., Bailyn, C. D., Mandel, I., \& Kalogera, V. 2011, ApJ, 741, 103

Farrar, G. R., \& Gruzinov, A. 2009, ApJ, 693, 329

Ferrarese, L. 2002, ApJ, 578, 90

Ferrarese, L., \& Ford, H. 2005, Space Sci. Rev., 116, 523

Ferrarese, L., \& Merritt, D. 2000, ApJ, 539, L9

Fishbone, L. G. 1973, ApJ, 185, 43

Fong, W., Berger, E., \& Fox, D. B. 2010, ApJ, 708, 9

Fong, W., et al. 2012, ApJ, 756, 189

Foucart, F. 2012, Phys. Rev. D, 86, 124007

Foucart, F., Duez, M. D., Kidder, L. E., \& Teukolsky, S. A. 2011, Phys. Rev. D, 83,024005

Fox, D. B., et al. 2005, Nature, 437, 845

Fragile, P. C. 2008, in Microquasars and Beyond

Fragile, P. C., \& Blaes, O. M. 2008, ApJ, 687, 757

Fragile, P. C., Blaes, O. M., Anninos, P., \& Salmonson, J. D. 2007, ApJ, 668, 417

Franceschini, A., Vercellone, S., \& Fabian, A. C. 1998, MNRAS, 297, 817

Frank, J., \& Rees, M. J. 1976, MNRAS, 176, 633

Frolov, V. P., Khokhlov, A. M., Novikov, I. D., \& Pethick, C. J. 1994, ApJ, 432, 680

Gebhardt, K., et al. 2000, AJ, 119, 1157

Gehrels, N., et al. 2005, Nature, 437, 851

Gezari, S., Halpern, J. P., Komossa, S., Grupe, D., \& Leighly, K. M. 2003, ApJ, 592,42

Gezari, S., et al. 2006, ApJ, 653, L25

—. 2008, ApJ, 676, 944 


\section{REFERENCES}

—. 2009, ApJ, 698, 1367

-. 2012, Nature, 485,217

Ghez, A. M., Salim, S., Hornstein, S. D., Tanner, A., Lu, J. R., Morris, M., Becklin, E. E., \& Duchêne, G. 2005, ApJ, 620, 744

Ghez, A. M., et al. 2008, ApJ, 689, 1044

Giannios, D., \& Metzger, B. D. 2011, MNRAS, 416, 2102

Gillessen, S., Eisenhauer, F., Trippe, S., Alexander, T., Genzel, R., Martins, F., \& Ott, T. 2009, ApJ, 692, 1075

Gillessen, S., et al. 2012, Nature, 481, 51

—. 2013, ApJ, 763, 78

Gomboc, A., \& Čadež, A. 2005, ApJ, 625, 278

González, J. A., Sperhake, U., Brügmann, B., Hannam, M., \& Husa, S. 2007, Physical Review Letters, 98, 091101

Goodman, J., \& Tan, J. C. 2004, ApJ, 608, 108

Gou, L., et al. 2011, ApJ, 742, 85

Graham, A. W., Onken, C. A., Athanassoula, E., \& Combes, F. 2011, MNRAS, 412,2211

Greene, J. E., \& Ho, L. C. 2007, ApJ, 667, 131

Greenhill, L. J., Moran, J. M., \& Herrnstein, J. R. 1997, ApJ, 481, L23

Greiner, J., Schwarz, R., Zharikov, S., \& Orio, M. 2000, A\&A, 362, L25

Grupe, D., Beuermann, K., Mannheim, K., Bade, N., Thomas, H.-C., de Martino, D., \& Schwope, A. 1995, A\&A, 299, L5

Grupe, D., Thomas, H.-C., \& Leighly, K. M. 1999, A\&A, 350, L31

Gualandris, A., \& Merritt, D. 2008, ApJ, 678, 780

Guillochon, J., \& Ramirez-Ruiz, E. 2013, ApJ, 767, 25

Guillochon, J., Ramirez-Ruiz, E., Rosswog, S., \& Kasen, D. 2009, ApJ, 705, 844

Haas, R., Shcherbakov, R. V., Bode, T., \& Laguna, P. 2012, ApJ, 749, 117 


\section{REFERENCES}

Halpern, J. P., Gezari, S., \& Komossa, S. 2004, ApJ, 604, 572

Hayasaki, K., Mineshige, S., \& Sudou, H. 2007, PASJ, 59, 427

Hayasaki, K., Stone, N., \& Loeb, A. 2012, ArXiv e-prints

Hazard, C., Mackey, M. B., \& Shimmins, A. J. 1963, Nature, 197, 1037

Herrnstein, J. R., et al. 1999, Nature, 400, 539

Hills, J. G. 1975, Nature, 254, 295

-. 1988, Nature, 331, 687

Hjorth, J., et al. 2005, Nature, 437, 859

Hoffman, L., \& Loeb, A. 2007, MNRAS, 377, 957

Holz, D. E., \& Hughes, S. A. 2005, ApJ, 629, 15

Hopkins, P. F., Hernquist, L., Cox, T. J., Di Matteo, T., Robertson, B., \& Springel, V. 2006, ApJS, 163, 1

Hopkins, P. F., Richards, G. T., \& Hernquist, L. 2007, ApJ, 654, 731

Hopkins, P. F., et al. 2010, ApJ, 715, 202

Hopman, C., \& Alexander, T. 2006, ApJ, 645, 1152

Hoyle, F., \& Fowler, W. A. 1963, MNRAS, 125, 169

Hunter, C., \& Qian, E. 1993, MNRAS, 262, 401

Ivanov, P. B., \& Chernyakova, M. A. 2006, A\&A, 448, 843

Ivanov, P. B., Chernyakova, M. A., \& Novikov, I. D. 2003, MNRAS, 338, 147

Ivanov, P. B., \& Novikov, I. D. 2001, ApJ, 549, 467

Ivanov, P. B., Polnarev, A. G., \& Saha, P. 2005, MNRAS, 358, 1361

Jahnke, K., \& Macciò, A. V. 2011, ApJ, 734, 92

Jenet, F. A., Lommen, A., Larson, S. L., \& Wen, L. 2004, ApJ, 606, 799

Jennrich, O. 2009, Classical and Quantum Gravity, 26, 153001

Johannsen, T., \& Psaltis, D. 2010, ApJ, 716, 187 


\section{REFERENCES}

—. 2012, ArXiv e-prints

Kalogera, V. 2000, ApJ, 541, 319

Kasen, D., \& Ramirez-Ruiz, E. 2010, ApJ, 714, 155

Kaspi, S., Smith, P. S., Netzer, H., Maoz, D., Jannuzi, B. T., \& Giveon, U. 2000, ApJ, 533, 631

Kesden, M. 2012a, Phys. Rev. D, 86, 064026

—. 2012b, Phys. Rev. D, 85, 024037

Kesden, M., Sperhake, U., \& Berti, E. 2010, ApJ, 715, 1006

Khokhlov, A., Novikov, I. D., \& Pethick, C. J. 1993a, ApJ, 418, 181

—. 1993b, ApJ, 418, 163

King, A. R., Lubow, S. H., Ogilvie, G. I., \& Pringle, J. E. 2005, MNRAS, 363, 49

Kippenhahn, R., \& Weigert, A. 1994, Stellar Structure and Evolution

Kiziltan, B., Kottas, A., \& Thorsett, S. E. 2010, ArXiv e-prints

Klein, A., Jetzer, P., \& Sereno, M. 2009, Phys. Rev. D, 80, 064027

Kobayashi, S., Laguna, P., Phinney, E. S., \& Mészáros, P. 2004, ApJ, 615, 855

Kochanek, C. S. 1994, ApJ, 422, 508

Kocsis, B., \& Loeb, A. 2008, Physical Review Letters, 101, 041101

Kocsis, B., \& Tremaine, S. 2011, MNRAS, 412, 187

Komossa, S. 2002, in Reviews in Modern Astronomy, Vol. 15, Reviews in Modern Astronomy, ed. R. E. Schielicke, 27

Komossa, S. 2004, in IAU Symposium, Vol. 222, The Interplay Among Black Holes, Stars and ISM in Galactic Nuclei, ed. T. Storchi-Bergmann, L. C. Ho, \& H. R. Schmitt, $45-48$

Komossa, S., \& Bade, N. 1999, A\&A, 343, 775

Komossa, S., \& Greiner, J. 1999, A\&A, 349, L45

Komossa, S., Halpern, J., Schartel, N., Hasinger, G., Santos-Lleo, M., \& Predehl, P. 2004, ApJ, 603, L17 


\section{REFERENCES}

Komossa, S., \& Merritt, D. 2008, ApJ, 683, L21

Komossa, S., Zhou, H., \& Lu, H. 2008, ApJ, 678, L81

Komossa, S., et al. 2009, ApJ, 701, 105

Kormendy, J., \& Bender, R. 2011, Nature, 469, 377

Kormendy, J., Fisher, D. B., Cornell, M. E., \& Bender, R. 2009, ApJS, 182, 216

Kormendy, J., \& Richstone, D. 1995, ARA\&A, 33, 581

Kouveliotou, C., Meegan, C. A., Fishman, G. J., Bhat, N. P., Briggs, M. S.,

Koshut, T. M., Paciesas, W. S., \& Pendleton, G. N. 1993, ApJ, 413, L101

Kouwenhoven, M. B. N., Brown, A. G. A., Portegies Zwart, S. F., \& Kaper, L. 2007, A\&A, 474, 77

Krolik, J. H. 1999, Active galactic nuclei : from the central black hole to the galactic environment

Kruger, A. T., Loredo, T. J., \& Wasserman, I. 2002, ApJ, 576, 932

Kumar, S., \& Pringle, J. E. 1985, MNRAS, 213, 435

Laguna, P., Miller, W. A., Zurek, W. H., \& Davies, M. B. 1993, ApJ, 410, L83

Larwood, J. D., Nelson, R. P., Papaloizou, J. C. B., \& Terquem, C. 1996, MNRAS, 282,597

Lauer, T. R., et al. 1995, AJ, 110, 2622

-. 2005, AJ, 129, 2138

—. 2007, ApJ, 664, 226

Lee, H. K., Wijers, R. A. M. J., \& Brown, G. E. 2000, Phys. Rep., 325, 83

Lei, W. H., Wang, D. X., Gong, B. P., \& Huang, C. Y. 2007, A\&A, 468, 563

Lei, W.-H., \& Zhang, B. 2011, ApJ, 740, L27

Levan, A. J., et al. 2011, Science, 333, 199

Li, G., \& Loeb, A. 2013, MNRAS, 429, 3040

Lidskii, V. V., \& Ozernoi, L. M. 1979, Soviet Astronomy Letters, 5, 16 


\section{REFERENCES}

Lightman, A. P., \& Shapiro, S. L. 1977, ApJ, 211, 244

Lippai, Z., Frei, Z., \& Haiman, Z. 2008, ApJ, 676, L5

Liu, F. K., Li, S., \& Chen, X. 2009, ApJ, 706, L133

Liu, T., Liang, E.-W., Gu, W.-M., Zhao, X.-H., Dai, Z.-G., \& Lu, J.-F. 2010, A\&A, 516, A16

Lodato, G., King, A. R., \& Pringle, J. E. 2009, MNRAS, 392, 332

Lodato, G., \& Pringle, J. E. 2006, MNRAS, 368, 1196

Lodato, G., \& Rossi, E. M. 2011, MNRAS, 410, 359

Loeb, A. 2007, Physical Review Letters, 99, 041103

Loeb, A., \& Rasio, F. A. 1994, ApJ, 432, 52

Loeb, A., \& Ulmer, A. 1997, ApJ, 489, 573

Lousto, C. O., Campanelli, M., Zlochower, Y., \& Nakano, H. 2010a, Classical and Quantum Gravity, 27, 114006

Lousto, C. O., Nakano, H., Zlochower, Y., \& Campanelli, M. 2010b, Phys. Rev. D, 81,084023

Lousto, C. O., \& Zlochower, Y. 2011, Physical Review Letters, 107, 231102

—. 2012, ArXiv e-prints

LSST Science Collaboration et al. 2009, ArXiv e-prints

Lucy, L. B. 1977, AJ, 82, 1013

Luminet, J.-P., \& Carter, B. 1986, ApJS, 61, 219

Luminet, J.-P., \& Marck, J.-A. 1985, MNRAS, 212, 57

Luminet, J.-P., \& Pichon, B. 1989, A\&A, 209, 85

Lynden-Bell, D. 1969, Nature, 223, 690

Madau, P., \& Quataert, E. 2004, ApJ, 606, L17

Madau, P., \& Rees, M. J. 2001, ApJ, 551, L27

Madigan, A.-M., Hopman, C., \& Levin, Y. 2011, ApJ, 738, 99 


\section{REFERENCES}

Magorrian, J., \& Tremaine, S. 1999, MNRAS, 309, 447

Maksym, W. P., Ulmer, M. P., \& Eracleous, M. 2010, ApJ, 722, 1035

Marck, J.-A. 1983a, Physics Letters A, 97, 140

—. 1983b, Royal Society of London Proceedings Series A, 385, 431

Marconi, A., \& Hunt, L. K. 2003, ApJ, 589, L21

Massi, M., Ros, E., \& Zimmermann, L. 2012, ArXiv e-prints

Mayer, L., Kazantzidis, S., Escala, A., \& Callegari, S. 2010, Nature, 466, 1082

Mayer, L., Kazantzidis, S., Madau, P., Colpi, M., Quinn, T., \& Wadsley, J. 2007,

Science, 316, 1874

McClintock, J. E., et al. 2011, Classical and Quantum Gravity, 28, 114009

McKinney, J. C., \& Narayan, R. 2007, MNRAS, 375, 513

McWilliams, S. T., Thorpe, J. I., Baker, J. G., \& Kelly, B. J. 2010, Phys. Rev. D, 81,064014

Merritt, D. 2006, ApJ, 648, 976

Merritt, D., Alexander, T., Mikkola, S., \& Will, C. M. 2010, Phys. Rev. D, 81, 062002

—. 2011, Phys. Rev. D, 84, 044024

Merritt, D., Mikkola, S., \& Szell, A. 2007, ApJ, 671, 53

Merritt, D., \& Milosavljević, M. 2005, Living Reviews in Relativity, 8, 8

Merritt, D., \& Poon, M. Y. 2004, ApJ, 606, 788

Merritt, D., Schnittman, J. D., \& Komossa, S. 2009, ApJ, 699, 1690

Merritt, D., \& Wang, J. 2005, ApJ, 621, L101

Meszaros, P., \& Rees, M. J. 1992, MNRAS, 257, 29P

Metzger, B. D., \& Berger, E. 2012, ApJ, 746, 48

Metzger, B. D., Giannios, D., \& Mimica, P. 2012, MNRAS, 420, 3528

Metzger, B. D., Piro, A. L., \& Quataert, E. 2008, MNRAS, 390, 781 


\section{REFERENCES}

—. 2009, MNRAS, 396, 304

Meyer, L., et al. 2012, Science, 338, 84

Mihos, J. C., \& Hernquist, L. 1996, ApJ, 464, 641

Milosavljević, M., \& Merritt, D. 2001, ApJ, 563, 34

Milosavljević, M., \& Phinney, E. S. 2005, ApJ, 622, L93

Miralda-Escudé, J., \& Kollmeier, J. A. 2005, ApJ, 619, 30

Montesinos Armijo, M., \& de Freitas Pacheco, J. A. 2011, ApJ, 736, 126

Mortlock, D. J., et al. 2011, Nature, 474, 616

Mościbrodzka, M., Shiokawa, H., Gammie, C. F., \& Dolence, J. C. 2012, ApJ, 752,

L1

Murray-Clay, R. A., \& Loeb, A. 2012, Nature Communications, 3

Navarro, J. F., Frenk, C. S., \& White, S. D. M. 1997, ApJ, 490, 493

Nolthenius, R. A., \& Katz, J. I. 1982, ApJ, 263, 377

Novikov, I. D., Pethick, C. J., \& Polnarev, A. G. 1992, MNRAS, 255, 276

Oesch, P. A., et al. 2010, ApJ, 709, L21

Ogilvie, G. I. 1999, MNRAS, 304, 557

Ohsuga, K., \& Mineshige, S. 2011, ApJ, 736, 2

Ohsuga, K., Mori, M., Nakamoto, T., \& Mineshige, S. 2005, ApJ, 628, 368

Okazaki, A. T., Bate, M. R., Ogilvie, G. I., \& Pringle, J. E. 2002, MNRAS, 337, 967

O'Leary, R. M., \& Loeb, A. 2009, MNRAS, 395, 781

—. 2012, MNRAS, 421, 2737

Öpik, E. 1924, Publications of the Tartu Astrofizica Observatory, 25, 1

Ostriker, E. C. 1999, ApJ, 513, 252

Özel, F., Psaltis, D., Narayan, R., \& McClintock, J. E. 2010, ApJ, 725, 1918 


\section{REFERENCES}

Paczynski, B. 1991, Acta Astronomica, 41, 257

Paczyńsky, B., \& Wiita, P. J. 1980, A\&A, 88, 23

Palenzuela, C., Garrett, T., Lehner, L., \& Liebling, S. L. 2010, Phys. Rev. D, 82, 044045

Pani, P., Cardoso, V., Gualtieri, L., Berti, E., \& Ishibashi, A. 2012, Physical

Review Letters, 109, 131102

Papaloizou, J. C. B., \& Lin, D. N. C. 1995, ApJ, 438, 841

Papaloizou, J. C. B., \& Pringle, J. E. 1983, MNRAS, 202, 1181

Papaloizou, J. C. B., \& Terquem, C. 1995, MNRAS, 274, 987

Peng, C. Y. 2007, ApJ, 671, 1098

Perets, H. B., \& Alexander, T. 2008, ApJ, 677, 146

Perets, H. B., Hopman, C., \& Alexander, T. 2007, ApJ, 656, 709

Pfahl, E., \& Loeb, A. 2004, ApJ, 615, 253

Phinney, E. S. 1989, in IAU Symposium, Vol. 136, The Center of the Galaxy, ed. M. Morris, 543

Pierre Auger Collaboration et al. 2008, Astroparticle Physics, 29, 188

Poon, M. Y., \& Merritt, D. 2004, ApJ, 606, 774

Portegies Zwart, S. F., Lee, C.-H., \& Lee, H. K. 1999, ApJ, 520, 666

Preece, R. D., Briggs, M. S., Mallozzi, R. S., Pendleton, G. N., Paciesas, W. S., \& Band, D. L. 2000, ApJS, 126, 19

Press, W. H., \& Teukolsky, S. A. 1977, ApJ, 213, 183

Press, W. H., Teukolsky, S. A., Vetterling, W. T., \& Flannery, B. P. 2002,

Numerical recipes in $\mathrm{C}++$ : the art of scientific computing

Pretorius, F. 2005, Physical Review Letters, 95, 121101

Quataert, E., \& Kasen, D. 2012, MNRAS, 419, L1

Ramirez-Ruiz, E., \& Rosswog, S. 2009, ApJ, 697, L77

Rauch, K. P., \& Ingalls, B. 1998, MNRAS, 299, 1231 


\section{REFERENCES}

Rauch, K. P., \& Tremaine, S. 1996, New Astronomy, 1, 149

Rees, M. J. 1988, Nature, 333, 523

Reynolds, C. S. 2013, ArXiv e-prints

Reynolds, C. S., Young, A. J., Begelman, M. C., \& Fabian, A. C. 1999, ApJ, 514, 164

Reynoso, M. M., Romero, G. E., \& Sampayo, O. A. 2006, A\&A, 454, 11

Roland, J., Frossati, G., \& Teyssier, R. 1994, A\&A, 290, 364

Romero, G. E., Reynoso, M. M., \& Christiansen, H. R. 2010, A\&A, 524, A4

Rosswog, S., Ramirez-Ruiz, E., \& Hix, W. R. 2009, ApJ, 695, 404

Rowlinson, A., et al. 2010, MNRAS, 409, 531

Ruffert, M., \& Janka, H.-T. 1999, A\&A, 344, 573

Ryan, F. D. 1995, Phys. Rev. D, 52, 5707

Saitoh, T. R., et al. 2012, ArXiv e-prints

Salpeter, E. E. 1964, ApJ, 140, 796

Sari, R., Kobayashi, S., \& Rossi, E. M. 2010, ApJ, 708, 605

Saxton, R. D., Read, A. M., Esquej, P., Komossa, S., Dougherty, S., Rodriguez-

Pascual, P., \& Barrado, D. 2012, A\&A, 541, A106

Sazhin, M. V. 1978, Soviet Ast., 22, 36

Schartmann, M., Burkert, A., Alig, C., Gillessen, S., Genzel, R., Eisenhauer, F., \& Fritz, T. K. 2012, ApJ, 755, 155

Schmidt, M. 1963, Nature, 197, 1040

Schnittman, J. D. 2007, ApJ, 667, L133

—. 2010, ApJ, 724, 39

-. 2011, Classical and Quantum Gravity, 28, 094021

Schnittman, J. D., \& Buonanno, A. 2007, ApJ, 662, L63

Schnittman, J. D., \& Krolik, J. H. 2008, ApJ, 684, 835 


\section{REFERENCES}

Schödel, R., et al. 2002, Nature, 419, 694

Schutz, B. F. 1986, Nature, 323, 310

-. 2009, Classical and Quantum Gravity, 26, 094020

Schwarzschild, M. 1979, ApJ, 232, 236

Sesana, A. 2012, Advances in Astronomy, 2012

Sesana, A., \& Vecchio, A. 2010, Phys. Rev. D, 81, 104008

Sesana, A., Vecchio, A., \& Volonteri, M. 2009, MNRAS, 394, 2255

Seto, N., \& Muto, T. 2010, Phys. Rev. D, 81, 103004

—. 2011, MNRAS, 415, 3824

Shakura, N. I., \& Sunyaev, R. A. 1973, A\&A, 24, 337

Shankar, F., Salucci, P., Granato, G. L., De Zotti, G., \& Danese, L. 2004, MNRAS, 354,1020

Shields, G. A., \& Bonning, E. W. 2008, ApJ, 682, 758

Shields, G. A., et al. 2009, ApJ, 707, 936

Shlosman, I., \& Begelman, M. C. 1987, Nature, 329, 810

Sijacki, D., Springel, V., \& Haehnelt, M. G. 2011, MNRAS, 414, 3656

Silk, J., \& Rees, M. J. 1998, A\&A, 331, L1

Soderberg, A. M., et al. 2006, ApJ, 650, 261

Soltan, A. 1982, MNRAS, 200, 115

Sopuerta, C. F. 2010, GW Notes, Vol. 4, p. 3-47, 4, 3

Spergel, D. N., et al. 2007, ApJS, 170, 377

Spitzer, L. 1987, Dynamical evolution of globular clusters

Stacy, A., Greif, T. H., \& Bromm, V. 2010, MNRAS, 403, 45

Steiner, J. F., McClintock, J. E., \& Reid, M. J. 2012, ApJ, 745, L7

Stone, N., \& Loeb, A. 2011, MNRAS, 412, 75 


\section{REFERENCES}

—. 2012a, Physical Review Letters, 108, 061302

—. 2012b, MNRAS, 422, 1933

Stone, N., Loeb, A., \& Berger, E. 2012a, ArXiv e-prints

Stone, N., Sari, R., \& Loeb, A. 2012b, ArXiv e-prints

Strubbe, L. E., \& Quataert, E. 2009, MNRAS, 400, 2070

—. 2011, MNRAS, 415, 168

Sun, M.-Y., Liu, T., Gu, W.-M., \& Lu, J.-F. 2012, ApJ, 752, 31

Syer, D., Clarke, C. J., \& Rees, M. J. 1991, MNRAS, 250, 505

Syer, D., \& Ulmer, A. 1999, MNRAS, 306, 35

Takahashi, T., et al. 2010, in Society of Photo-Optical Instrumentation Engineers (SPIE) Conference Series, Vol. 7732, Society of Photo-Optical Instrumentation Engineers (SPIE) Conference Series

Toomre, A. 1964, ApJ, 139, 1217

Tremaine, S., et al. 2002, ApJ, 574, 740

Ulmer, A. 1999, ApJ, 514, 180

Ulmer, A., Paczynski, B., \& Goodman, J. 1998, A\&A, 333, 379

Valentim, R., Rangel, E., \& Horvath, J. E. 2011, MNRAS, 414, 1427

van Velzen, S., Frail, D. A., Körding, E., \& Falcke, H. 2013, A\&A, 552, A5

van Velzen, S., Körding, E., \& Falcke, H. 2011a, MNRAS, 417, L51

van Velzen, S., et al. 2011b, ApJ, 741, 73

Vasiliev, E., \& Merritt, D. 2013, ArXiv e-prints

Vereshchagin, S., Tutukov, A., Iungelson, L., Kraicheva, Z., \& Popova, E. 1988, Ap\&SS, 142, 245

Volonteri, M., Haardt, F., \& Madau, P. 2003, ApJ, 582, 559

Volonteri, M., Lodato, G., \& Natarajan, P. 2008, MNRAS, 383, 1079

Volonteri, M., Madau, P., Quataert, E., \& Rees, M. J. 2005, ApJ, 620, 69 


\section{REFERENCES}

Volonteri, M., \& Rees, M. J. 2006, ApJ, 650, 669

Wald, R. M. 1984, General relativity

Wandel, A., Peterson, B. M., \& Malkan, M. A. 1999, ApJ, 526, 579

Wang, J., \& Merritt, D. 2004, ApJ, 600, 149

Wang, T.-G., Zhou, H.-Y., Komossa, S., Wang, H.-Y., Yuan, W., \& Yang, C. 2012, ApJ, 749, 115

Wang, T.-G., Zhou, H.-Y., Wang, L.-F., Lu, H.-L., \& Xu, D. 2011, ApJ, 740, 85

Wegg, C. 2012, ApJ, 749, 183

Wegg, C., \& Nate Bode, J. 2011, ApJ, 738, L8

Wetzel, A. R., \& White, M. 2010, MNRAS, 403, 1072

Wheeler, J. 1971, in Study Week on Nuclei of Galaxies, ed. D. J. K. O'Connell, 539

Willems, B., Andrews, J., Kalogera, V., \& Belczynski, K. 2008, in American Institute of Physics Conference Series, Vol. 983, 40 Years of Pulsars: Millisecond Pulsars, Magnetars and More, ed. C. Bassa, Z. Wang, A. Cumming, \& V. M. Kaspi, 464-468

Wyithe, J. S. B., \& Loeb, A. 2003, ApJ, 595, 614

Yu, Q., \& Tremaine, S. 2001, AJ, 121, 1736

—. 2002, MNRAS, 335, 965

—. 2003, ApJ, 599, 1129

Zalamea, I., Menou, K., \& Beloborodov, A. M. 2010, MNRAS, 409, L25

Zauderer, B. A., Berger, E., Margutti, R., Pooley, G. G., Sari, R., Soderberg, A. M., Brunthaler, A., \& Bietenholz, M. F. 2013, ApJ, 767, 152

Zauderer, B. A., et al. 2011, Nature, 476, 425

Zhao, H., Haehnelt, M. G., \& Rees, M. J. 2002, New Astronomy, 7, 385 


\section{Appendix A}

\section{The Parabolic Hill Equations}

Consider the parabolic restricted three body problem, where the central mass $M_{\mathrm{BH}} \gg M_{*}$ and $M_{*}=m_{1}+m_{2}$. Here the smaller bodies of masses $m_{1}$ and $m_{2}$ represent pieces of the star undergoing tidal disruption. The equations of motion for this system are derived in $\S 2$ of SKR10; we present the derivation here for completeness. These differential equations do not make assumptions about the relative masses of $m_{1}$ and $m_{2}$.

If we center our coordinate system on the $\mathrm{SMBH}$, and define position vectors $\vec{r}_{1}$ and $\overrightarrow{r_{2}}$ for the smaller particles, we can write the Newtonian equations of motion:

$$
\begin{aligned}
& \ddot{\vec{r}}_{1}=-\frac{G M_{\mathrm{BH}}}{r_{1}^{3}} \vec{r}_{1}+\frac{G m_{2}}{\left|\vec{r}_{1}-\vec{r}_{2}\right|^{3}}\left(\vec{r}_{2}-\vec{r}_{1}\right) \\
& \ddot{\overrightarrow{r_{2}}}=-\frac{G M_{\mathrm{BH}}}{r_{2}^{3}} \vec{r}_{2}-\frac{G m_{1}}{\left|\vec{r}_{1}-\vec{r}_{2}\right|^{3}}\left(\vec{r}_{2}-\vec{r}_{1}\right) .
\end{aligned}
$$

Defining $\vec{r}=\vec{r}_{2}-\vec{r}_{1}$, we can then write

$$
\ddot{\vec{r}}=-\frac{G M_{\mathrm{BH}}}{r_{2}^{3}} \vec{r}_{2}+\frac{G M_{\mathrm{BH}}}{r_{1}^{3}} \vec{r}_{1}-\frac{G M_{*}}{r^{3}} \vec{r} .
$$

Next, we assume that both of the smaller bodies are roughly following a parabolic center of mass trajectory,

$$
\vec{R}=\frac{2 R_{\mathrm{p}}}{1+\cos f}(\cos f, \sin f, 0),
$$

so we can linearize Eqs. A.1, A.2 as $\vec{r}_{1} \approx \vec{R}+\delta \vec{r}_{1}, \vec{r}_{2} \approx \vec{R}+\delta \vec{r}_{2}$ (assuming $\delta r_{1,2} \ll R$ ) to find

$$
\ddot{\vec{r}}=\frac{G M_{\mathrm{BH}}}{R^{3}}\left(-\vec{r}+\frac{3 \vec{r} \cdot \vec{R}}{R^{2}} \vec{R}\right)-\frac{G M_{*}}{r^{3}} \vec{r} .
$$

Here we have discarded all terms quadratic or higher in $\delta r_{1,2}$. Separating this equation of motion into components gives us three ordinary differential equations for the coordinate 
motion of each small body. Following the unit convention of $\S 3$, where tildes denote distances normalized by $R_{*}$ and times normalized by $\sqrt{R_{*}^{3} /\left(G M_{*}\right)}$, these parabolic Hill equations are

$$
\begin{aligned}
& \ddot{\tilde{x}}= \beta^{3} \frac{(1+\cos f)^{3}}{8}[-\tilde{x}+3(\tilde{x} \cos f+\tilde{y} \sin f) \cos f] \\
&-\frac{\tilde{x}}{\left(\tilde{x}^{2}+\tilde{y}^{2}+\tilde{z}^{2}\right)^{3 / 2}} \\
& \ddot{\tilde{y}}= \beta^{3} \frac{(1+\cos f)^{3}}{8}[-\tilde{y}+3(\tilde{x} \cos f+\tilde{y} \sin f) \sin f] \\
&-\frac{\tilde{y}}{\left(\tilde{x}^{2}+\tilde{y}^{2}+\tilde{z}^{2}\right)^{3 / 2}} \\
& \ddot{\tilde{z}}=-\beta^{3} \frac{(1+\cos f)^{3}}{8} \tilde{z}-\frac{\tilde{z}}{\left(\tilde{x}^{2}+\tilde{y}^{2}+\tilde{z}^{2}\right)^{3 / 2}} .
\end{aligned}
$$

Dropping the last term (which gives the interaction between the masses $m_{1}$ and $m_{2}$ ) in each of these three equations allows the six "free solutions" to the parabolic Hill equations to be found analytically. These solutions are given in Eqs. 4.8 and 4.14, and represent the motion of a cloud of test particles in tidal free fall, if $m_{1}$ is taken to be a particle located at $\tilde{x}=\tilde{y}=\tilde{z}=0$, and $m_{2}$ is a particle located anywhere else in the cloud.

Each of the six free solutions can be thought of as the perturbation to a single orbital element of the parabolic center of mass trajectory. Specifically, the in-plane solutions vary longitude of pericenter (A), time of pericenter (B), variation in the pericenter distance $(\mathrm{C})$, and variation in eccentricity given a fixed pericenter (D). The out of plane solutions represent rotations around the apsidal line $(\mathrm{E})$ and latus rectum $(\mathrm{F})$. 


\section{Appendix B}

\section{Principal Axes of Free Solutions}

Using the in-plane free solutions, we find that the initial phases of the principal axes at $f=f_{\mathrm{c}}$ are given by

$$
\cos \theta_{\mathrm{ex}}= \pm \sqrt{\frac{1}{2} \pm \frac{j}{2 \sqrt{j^{2}+4 k^{2}}}}
$$

where

$$
\begin{aligned}
j= & -\frac{16}{25} \beta+\frac{148}{25}+\frac{1312}{25 \beta}-\frac{352}{5 \beta^{2}} \\
& +\sqrt{1-\beta^{-1}}\left(-\frac{16}{25} \beta+\frac{788}{25}-\frac{2208}{25 \beta}+\frac{32}{5 \beta^{2}}\right) \\
k= & \sqrt{\beta}\left(\frac{72}{25}-\frac{936}{25 \beta}+\frac{1144}{25 \beta^{2}}-\frac{16}{5 \beta^{3}}\right) \\
& +\sqrt{\beta-1}\left(\frac{72}{25}+\frac{216}{25 \beta}-\frac{176}{5 \beta^{2}}\right)
\end{aligned}
$$

are exact expressions. Note that this gives us 8 possible solutions for $\theta_{\mathrm{ex}}$. While 4 are spurious, the other 4 of these are valid, with each minimum (maximum) in $r_{\mathrm{H}}$ possessing an equal minimum (maximum) $180^{\circ}$ around the star's center of mass. In particular, for $\sin \theta_{0}>0$

$$
\cos \theta_{\min }=(-1)^{p} \sqrt{\frac{1}{2}+(-1)^{q} \frac{j}{2 \sqrt{j^{2}+4 k^{2}}}}
$$

and

$$
\cos \theta_{\max }=(-1)^{P} \sqrt{\frac{1}{2}+(-1)^{Q} \frac{j}{2 \sqrt{j^{2}+4 k^{2}}}} .
$$

Here the behavior of $\theta_{\min }$ and $\theta_{\max }$ is piecewise with respect to $\beta$, due to zeros of $j$ 


\section{APPENDIX B. PRINCIPAL AXES OF FREE SOLUTIONS}

and $k$. We can describe this behavior for all $\beta>1$ by setting

$$
\{p, q, P, Q\}=\left\{\begin{array}{l}
\{1,2,2,1\}, \beta<1.073 \\
\{2,1,1,2\}, 1.073 \leq \beta<4.944 \\
\{1,2,2,1\}, 4.944 \leq \beta
\end{array}\right.
$$

To gain better intuition for the geometry of these principal axes, we can Taylor expand $r_{\text {long }}=r_{\mathrm{H}}\left(\theta_{\max }\right)$ and $r_{\text {short }}=r_{\mathrm{H}}\left(\theta_{\min }\right)$ in the limit of large $\beta$ :

$$
\begin{aligned}
& r_{\text {long }}=\frac{4}{5} \beta^{1 / 2}+\frac{22}{5} \beta^{-1 / 2}+O\left(\beta^{-3 / 2}\right) \\
& r_{\text {short }}=2 \beta^{-1 / 2}-\frac{23}{2} \beta^{-3 / 2}+O\left(\beta^{-5 / 2}\right) .
\end{aligned}
$$

These results are poorly convergent for $\beta<10$, but describe the exact solutions well above this threshold. We are now in a position to derive approximate formulae for the angles presented in Fig. 4.3. By expanding the numerator and denominator of $\tan \psi_{\mathrm{c}}=-y_{\mathrm{H}}\left(\theta_{\max }\right) / x_{\mathrm{H}}\left(\theta_{\max }\right)$ we find

$$
\tan \psi_{\mathrm{c}} \approx \frac{16 \beta^{1 / 2}+86 \beta^{-1 / 2}}{8+185 \beta^{-1}} .
$$

Using trigonometric identities and the Keplerian expression $\tan \Upsilon_{\mathrm{c}}=\sqrt{\beta}+\sqrt{\beta-1} \approx$ $2 \beta^{1 / 2}\left(\Upsilon_{\mathrm{c}}\right.$ is the angle between the $\hat{x}$ and a parabolic orbit's velocity vector at $f=f_{\mathrm{c}}$ ), we find the misalignment angle

$$
\tan \nu_{\mathrm{c}} \approx \frac{284}{32 \beta^{3 / 2}+180 \beta^{1 / 2}+185 \beta^{-1 / 2}} .
$$

We have defined $\nu_{\mathrm{c}}$ as the (positive) angle between the center of mass velocity and the long principal axis at $f=f_{\mathrm{c}}$. It asymptotes to $0^{\circ}$ as $\beta$ goes to $\infty$. 


\section{Appendix C}

\section{Innermost Stable Spherical Orbits}

We follow the simple formalism of Perez-Giz 2013 (in prep.) to calculate the ISSO radius for Kerr metric geodesics, and present it here for completeness (in this appendix we use geometrized units, $\mathrm{G}=\mathrm{c}=1$, for radial distance $r$ and $\mathrm{BH}$ spin $a$ ). As mentioned in $\S 2$, the ISSO is the innermost stable orbit at constant radius, but fixed nonzero inclination around a Kerr BH. We define an inclination angle $\iota$ such that $C=\cos \iota$, the Carter constant $Q=L^{2} \sin ^{2} \iota$, and conserved vertical angular momentum $L_{\mathrm{z}}=L C$, i.e. $\iota=0$ corresponds to equatorial prograde orbits. Calculation of the equatorial plane ISCO, $r_{\text {ISCO }}$, is well-documented in the literature (Bardeen et al. 1972), and consists of finding the roots of the polynomial

$$
Z(r)=(r(r-6))^{2}-a^{2}\left(2 r(3 r+14)-9 a^{2}\right)=0,
$$

with one root the prograde and one the retrograde ISCO. The polar $(\iota= \pm \pi / 2)$ ISSO can be found at the root of

$$
\begin{aligned}
P(r)= & r^{3}\left(r^{2}(r-6)+a^{2}(3 r+4)\right) \\
& +a^{4}\left(3 r(r-2)+a^{2}\right)=0
\end{aligned}
$$

that lies between $r=6$ and $r=1+\sqrt{3}+\sqrt{3+2 \sqrt{3}}$. Finally, the generic ISSO is a root of the polynomial

$$
\begin{gathered}
S(r)=r^{8} Z(r)+a^{2}\left(1-C^{2}\right)\left(a^{2}\left(1-C^{2}\right) Y(r)\right. \\
\left.-2 r^{4} X(r)\right),
\end{gathered}
$$




\section{APPENDIX C. INNERMOST STABLE SPHERICAL ORBITS}

with auxiliary functions defined as

$$
\begin{aligned}
X(r)= & a^{2}\left(a^{2}\left(3 a^{2}+4 r(2 r-3)\right)\right. \\
& \left.+r^{2}(15 r(r-4)+28)\right)-6 r^{4}\left(r^{2}-4\right) \\
Y(r)= & a^{4}\left(a^{4}+r^{2}(7 r(3 r-4)+36)\right)+6 r(r-2) \\
& \times\left(a^{6}+2 r^{3}\left(a^{2}(3 r+2)+3 r^{2}(r-2)\right)\right) .
\end{aligned}
$$

Specifically, $r_{\text {ISSO }}$ is the root of $S(r)$ located between the appropriate $r_{\text {ISCO }}$ (prograde or retrograde) and the polar ISSO. 


\section{Appendix D}

\section{Post-Newtonian Merger Treatment}

The Lousto et al. (2010a) formulae discussed in $\S 3$ are presented in this appendix, along with a discussion of their applicability to BH-NS mergers. These formulae are calibrated to define the outcome of a $\mathrm{BH}-\mathrm{BH}$ merger, although we apply them more generally to the case of BH-NS mergers with masses $m_{1}$ and $m_{2}$, and mass ratio $q=m_{1} / m_{2} \leq 1$. We define the symmetric mass ratio $\eta=q /(1+q)^{2}$, and denote the dimensionless compact object spin vectors $\vec{a}_{\mathrm{i}}$ as having components $a_{\mathrm{i}}^{\|}$and $a_{\mathrm{i}}^{\perp}$, which are parallel to and perpendicular to the binary angular momentum, respectively. We further define $\Theta_{ \pm}$as the angle made between the radial direction and the vector $\vec{\Delta}_{ \pm}=\left(m_{1}+m_{2}\right)\left(m_{2} \vec{a}_{2} \pm m_{1} \vec{a}_{1}\right)$. Then the post-merger remnant mass is found to decrease by a fraction

$$
\begin{array}{r}
\delta M / M=\eta \tilde{E}_{\mathrm{ISCO}}+E_{2} \eta^{2}+E_{3} \eta^{3}+\frac{\eta^{2}}{(1+q)^{2}} \\
\times\left(E_{\mathrm{S}}\left(a_{2}^{\|}+q^{2} a_{1}^{\|}\right)+E_{\Delta}(1-q)\left(a_{2}^{\|}-q a_{1}^{\|}\right)\right. \\
\quad+E_{\mathrm{A}}\left|\vec{a}_{2}+q \vec{a}_{1}\right|^{2}+E_{\mathrm{B}}\left|a_{2}^{\perp}+q a_{1}^{\perp}\right|^{2} \\
\times\left(\cos ^{2}\left(\Theta_{+}-\Theta_{2}\right)+E_{\mathrm{C}}\right)+E_{\mathrm{D}}\left|\vec{a}_{2}-q \vec{a}_{1}\right|^{2} \\
\left.+E_{\mathrm{E}}\left|a_{2}^{\perp}-q a_{1}^{\perp}\right|^{2}\left(\cos ^{2}\left(\Theta_{-}-\Theta_{3}\right)+E_{\mathrm{F}}\right)\right) .
\end{array}
$$




\section{APPENDIX D. POST-NEWTONIAN MERGER TREATMENT}

Here the energy lost during the inspiral from infinity down to the plunge is fit as

$$
\begin{array}{r}
\tilde{E}_{\mathrm{ISCO}} \approx 1 \\
\times\left(q(1+2 q) a_{1}^{\|}+(2+q) a_{2}^{\|}\right)-\frac{\sqrt{8}}{324 \sqrt{2}(1+q)^{2}} \\
\times\left(\vec{a}_{2}^{2}-3\left(a_{2}^{\|}\right)^{2}-2 q\left(\vec{a}_{1} \cdot \vec{a}_{2}-3 a_{1}^{\|} a_{2}^{\|}\right)\right. \\
\left.+q^{2}\left(\vec{a}_{1}^{2}-3\left(a_{1}^{\|}\right)^{2}\right)\right) .
\end{array}
$$

The final spin vector of the post-merger $\mathrm{BH}$ is

$$
\begin{aligned}
& \vec{a}^{\prime}=\left(1-\frac{\delta M}{M}\right)^{-2}\left[\eta \tilde{\vec{J}}_{\mathrm{ISCO}}+\left(J_{2} \eta^{2}+J_{3} \eta^{3}\right) \hat{\vec{n}}_{\|}\right. \\
& +\frac{\eta^{2}}{(1+q)^{2}}\left(\left(J_{\mathrm{A}}\left(a_{2}^{\|}+q^{2} a_{1}^{\|}\right)+J_{\mathrm{B}}(1-q)\left(a_{2}^{\|}-q a_{1}^{\|}\right)\right) \hat{\vec{n}}_{\|}\right. \\
& +(1-q)\left|\vec{a}_{2}^{\perp}-q \vec{a}_{1}^{\perp}\right| \sqrt{J_{\Delta} \cos \left(2\left(\Theta_{\Delta}-\Theta_{4}\right)\right)+J_{\mathrm{M}}} \hat{\vec{n}}_{\perp} \\
& \left.\left.+\left|\vec{a}_{2}^{\perp}+q^{2} \vec{a}_{1}^{\perp}\right| \sqrt{J_{\mathrm{S}} \cos \left(2\left(\Theta_{\mathrm{S}}-\Theta_{5}\right)\right)+J_{\mathrm{MS}}} \hat{\vec{n}}_{\perp}\right)\right]
\end{aligned}
$$

where the angular momentum radiated during the inspiral from infinity to the plunge is

$$
\begin{array}{r}
\tilde{\vec{J}}_{\mathrm{ISCO}} \approx\left[2 \sqrt{3}-1.5255862 \eta-\frac{1}{9 \sqrt{2}(1+q)^{2}}\right. \\
\times\left(q(7+8 q) a_{1}^{\|}+(8+7 q) a_{2}^{\|}\right)+\frac{2}{9 \sqrt{3}(1+q)^{2}} \\
\times\left(\vec{a}_{2}^{2}-3\left(a_{2}^{\|}\right)^{2}-2 q\left(\vec{a}_{1} \cdot \vec{a}_{2}-3 a_{1}^{\|} a_{2}^{\|}\right)\right. \\
\left.\left.+q^{2}\left(\vec{a}_{1}^{2}-3\left(a_{1}^{\|}\right)^{2}\right)\right)\right] \hat{\vec{n}}_{\|}-\frac{1}{9 \sqrt{2}(1+q)^{2}} \\
\left(q(1+4 q) \vec{a}_{1}+(4+q) \vec{a}_{2}\right)+\frac{1}{\eta} \frac{\vec{a}_{2}+q^{2} \vec{a}_{1}}{(1+q)^{2}} .
\end{array}
$$

The other variables in these formulae are empirical fitting constants, with values found to be $E_{2}=.341, E_{3}=0.522, E_{\mathrm{S}}=0.673, E_{\Delta}=-0.3689, E_{\mathrm{A}}=-0.0136, E_{\mathrm{B}}=0.045, E_{\mathrm{C}}=$ $0, E_{\mathrm{D}}=0.2611, E_{\mathrm{E}}=0.0959, E_{\mathrm{F}}=0, J_{2}=-2.81, J_{3}=1.69, J_{\mathrm{A}}=-2.9667, J_{\mathrm{B}}=$ $-1.7296, J_{\Delta}=J_{\mathrm{M}}=J_{\mathrm{S}}=J_{\mathrm{MS}}=0$. We also set $\Theta_{2}=\Theta_{3}=\Theta_{4}=\Theta_{5}=0$ because of the weak dependence of the results on these parameters. With extremely low probability $\left(\sim 10^{-5}\right)$, Eq. (B3) can give super-extremal spin values, of $a^{\prime}>1$; we discard these cases from our Monte Carlo sample when they appear.

As mentioned in $\S 3$, these formulae, which were derived, and calibrated, for $\mathrm{BH}-\mathrm{BH}$ mergers, are found to give surprisingly good agreement with detailed results for BH-NS 
Table D.1:: In this table we compare the full NR results for post-merger $\mathrm{BH}$ spin $a_{\mathrm{BH}}^{\prime}$ to the predictions of our PN fitting formula, Eq. (D.3). Here $\psi$ is the initial spin-orbit misalignment angle, $q$ is the mass ratio, and the "Ref." column refers to the paper whose NR data we are comparing the PN results to. The agreement is quite strong for small $q$. For $q=1 / 3$, roughly the upper limit considered in our $\mathrm{BH}$ mass functions, the error is $\lesssim 10 \%$, which is still acceptable for our purposes. In the last two columns we also demonstrate the reasonable agreement between NR estimates for initial post-merger disk mass, $M_{\mathrm{d}}^{\mathrm{NR}}$, and the prescription of Eq. (7.6), $M_{\mathrm{dis}}^{\mathrm{F}}$. The disk mass fitting formula works very well for low and moderate values of spin, but becomes less accurate for the $a_{\mathrm{BH}}=0.9$ runs. In each simulation in this table, the NS mass is $1.4 M_{\odot}$.

\begin{tabular}{r||r|r|r|r|r|r|r|r} 
Ref. & $q$ & $\psi$ & $a_{\mathrm{BH}}$ & $R_{\mathrm{NS}}$ & $a_{\mathrm{BH}, \mathrm{NR}}^{\prime}$ & $a_{\mathrm{BH}, \mathrm{PN}}^{\prime}$ & $\frac{M_{\mathrm{dis}}^{\mathrm{NR}}}{M_{\mathrm{NS}}}$ & $\frac{M_{\mathrm{dis}}^{\mathrm{F}}}{M_{\mathrm{NS}}}$ \\
\hline (Foucart 2012) & $1 / 7$ & $0^{\circ}$ & 0.5 & $14.4 \mathrm{~km}$ & 0.67 & 0.658 & $\leq 0.4 \%$ & $0 \%$ \\
(Foucart 2012) & $1 / 7$ & $0^{\circ}$ & 0.7 & $14.4 \mathrm{~km}$ & 0.80 & 0.786 & $6 \%$ & $7.2 \%$ \\
(Foucart 2012) & $1 / 7$ & $0^{\circ}$ & 0.9 & $14.4 \mathrm{~km}$ & 0.92 & 0.913 & $28 \%$ & $22.9 \%$ \\
(Foucart 2012) & $1 / 5$ & $0^{\circ}$ & 0.5 & $14.4 \mathrm{~km}$ & 0.71 & 0.681 & $6 \%$ & $6.5 \%$ \\
(Foucart et al. 2011) & $1 / 7$ & $0^{\circ}$ & 0.9 & $12.2 \mathrm{~km}$ & 0.923 & 0.913 & $10 \%$ & $11.8 \%$ \\
(Foucart et al. 2011) & $1 / 7$ & $0^{\circ}$ & 0.9 & $13.3 \mathrm{~km}$ & 0.919 & 0.913 & $20 \%$ & $17.4 \%$ \\
(Foucart et al. 2011) & $1 / 7$ & $0^{\circ}$ & 0.9 & $14.4 \mathrm{~km}$ & 0.910 & 0.913 & $30 \%$ & $22.1 \%$ \\
(Foucart et al. 2011) & $1 / 7$ & $20^{\circ}$ & 0.9 & $14.4 \mathrm{~km}$ & 0.909 & 0.911 & $28 \%$ & $20.3 \%$ \\
(Foucart et al. 2011) & $1 / 7$ & $40^{\circ}$ & 0.9 & $14.4 \mathrm{~km}$ & 0.898 & 0.900 & $15 \%$ & $13.8 \%$ \\
(Foucart et al. 2011) & $1 / 7$ & $60^{\circ}$ & 0.9 & $14.4 \mathrm{~km}$ & 0.862 & 0.870 & $3 \%$ & $1.3 \%$ \\
(Foucart et al. 2011) & $1 / 3$ & $0^{\circ}$ & 0.0 & $14.6 \mathrm{~km}$ & 0.56 & 0.54 & $5.2 \%$ & $5.21 \%$ \\
(Foucart et al. 2011) & $1 / 3$ & $0^{\circ}$ & 0.5 & $14.6 \mathrm{~km}$ & 0.77 & 0.70 & $15.5 \%$ & $16.3 \%$ \\
(Foucart et al. 2011) & $1 / 3$ & $0^{\circ}$ & 0.9 & $14.6 \mathrm{~km}$ & 0.93 & 0.829 & $38.9 \%$ & $28.3 \%$ \\
(Foucart et al. 2011) & $1 / 3$ & $20^{\circ}$ & 0.5 & $14.6 \mathrm{~km}$ & 0.76 & 0.699 & $14.5 \%$ & $15.8 \%$ \\
(Foucart et al. 2011) & $1 / 3$ & $40^{\circ}$ & 0.5 & $14.6 \mathrm{~km}$ & 0.74 & 0.691 & $11.5 \%$ & $14.1 \%$ \\
(Foucart et al. 2011) & $1 / 3$ & $60^{\circ}$ & 0.5 & $14.6 \mathrm{~km}$ & 0.71 & 0.671 & $8.0 \%$ & $11.4 \%$ \\
(Foucart et al. 2011) & $1 / 3$ & $80^{\circ}$ & 0.5 & $14.6 \mathrm{~km}$ & 0.66 & 0.636 & $6.1 \%$ & $8.0 \%$
\end{tabular}


mergers simulated in full NR. We demonstrate the agreement in Table II, where we also plot the generally good agreement between Eq. (7.6) and NR results for the initial mass of the post-merger accretion disk. The largest errors in the disk mass fitting formula seem to occur for large disk masses $\left(M_{\mathrm{dis}}>0.2 M_{\mathrm{NS}}\right)$, and for high pre-merger spin-orbit misalignments $\left(\psi \gtrsim 60^{\circ}\right)$. The first of these cases occurs only for a small subset of our BH-NS mergers; the second occurs for a larger fraction, and may result in a modest overestimate of $f_{\mathrm{GRB}}$. 